Article

\title{
Polyhalonitrobutadienes as Versatile Building Blocks for the Biotargeted Synthesis of Substituted N-Heterocyclic Compounds ${ }^{+}$
}

\author{
Viktor A. Zapol'skii ${ }^{1}$ (D, Ursula Bilitewski ${ }^{2}$, Sören R. Kupiec ${ }^{1}$, Isabell Ramming ${ }^{2}$ and \\ Dieter E. Kaufmann 1,*(D) \\ 1 Institute of Organic Chemistry, Clausthal University of Technology, Leibnizstraße 6, \\ 38678 Clausthal-Zellerfeld, Germany; viktor.zapolskii@tu-clausthal.de (V.A.Z.); \\ SOEREN104@gmail.com (S.R.K.) \\ 2 Helmholtz Centre for Infection Research (HZI), Inhoffenstr. 7, 38124 Braunschweig, Germany; \\ ursula.bilitewski@helmholtz-hzi.de (U.B.); Isabell.Ramming@helmholtz-hzi.de (I.R.) \\ * Correspondence: dieter.kaufmann@tu-clausthal.de \\ + Chemistry of Polyhalogenated Nitrobutadienes, 16. Chemistry of Polyhalogenated Nitrobutadiens, 15. \\ Zapol'skii, V.A.; Namyslo, J.C.; Gjikaj, M.; Kaufmann, D.E. Synthesis of Novel 4-Nitro-3-amino-1H- \\ pyrazole-5-carbaldehydes and Pyrazolo[3,4-f]indazole-4,8- diones. Heterocycles 2016, 93, 628-646, \\ doi:10.3987/COM-15-S(T)46.
}

Received: 30 April 2020; Accepted: 17 June 2020; Published: 21 June 2020

\begin{abstract}
Substituted nitrogen heterocycles are structural key units in many important pharmaceuticals. A new synthetic approach towards heterocyclic compounds displaying antibacterial activity against Staphylococcus aureus or cytotoxic activity has been developed. The selective synthesis of a series of 64 new $\mathrm{N}$-heterocycles from the three nitrobutadienes 2-nitroperchloro-1,3-butadiene, 4-bromotetrachloro-2-nitro-1,3-butadiene and (Z)-1,1,4-trichloro-2,4-dinitrobuta-1,3-diene proved feasible. Their reactions with $\mathrm{N}-, \mathrm{O}$ - and S-nucleophiles provide rapid access to push-pull substituted benzoxazolines, benzimidazolines, imidazolidines, thiazolidinones, pyrazoles, pyrimidines, pyridopyrimidines, benzoquinolines, isothiazoles, dihydroisoxazoles, and thiophenes with unique substitution patterns. Antibacterial activities of 64 synthesized compounds were examined. Additionally, seven compounds (thiazolidinone, nitropyrimidine, indole, pyridopyrimidine, and thiophene derivatives) exhibited a significant cytotoxicity with $\mathrm{IC}_{50}$-values from 1.05 to $20.1 \mu \mathrm{M}$. In conclusion, it was demonstrated that polyhalonitrobutadienes have an interesting potential as structural backbones for a variety of highly functionalized, pharmaceutically active heterocycles.
\end{abstract}

Keywords: polyhalonitrobutadienes; nucleophilic substitution; heterocyclization; nitrogen heterocycles; medicinal chemistry

\section{Introduction}

Halogenated nitrobutadienes are part of a relatively small group of selectively reactive aliphatic nitro compounds [1]. Representatives with one or two nitro and three to five halogen groups are easily accessible by introduction of an activating and directing nitro group into polyhalo-1,3-butadienes [2]. These can be easily obtained in high purity and multigram scale by radical dimerization of industrial solvents such as trichloroethene and 1,2-dichloroethene with subsequent dehydrohalogenation-halogenation, followed by nitration reactions. 2-Nitroperchlorobutadiene (1) has been synthesized in three steps from trichloroethene [3,4] (Scheme 1). 4-Bromotetrachloro-2-nitrobutadiene (2) could be obtained from trichloroethene in five steps [5]. 
(Z)-1,1,4-Trichloro-2,4-dinitrobutadiene (3) was made accessible in four steps from a Z,E-mixture of 1,2-dichloroethene [6].
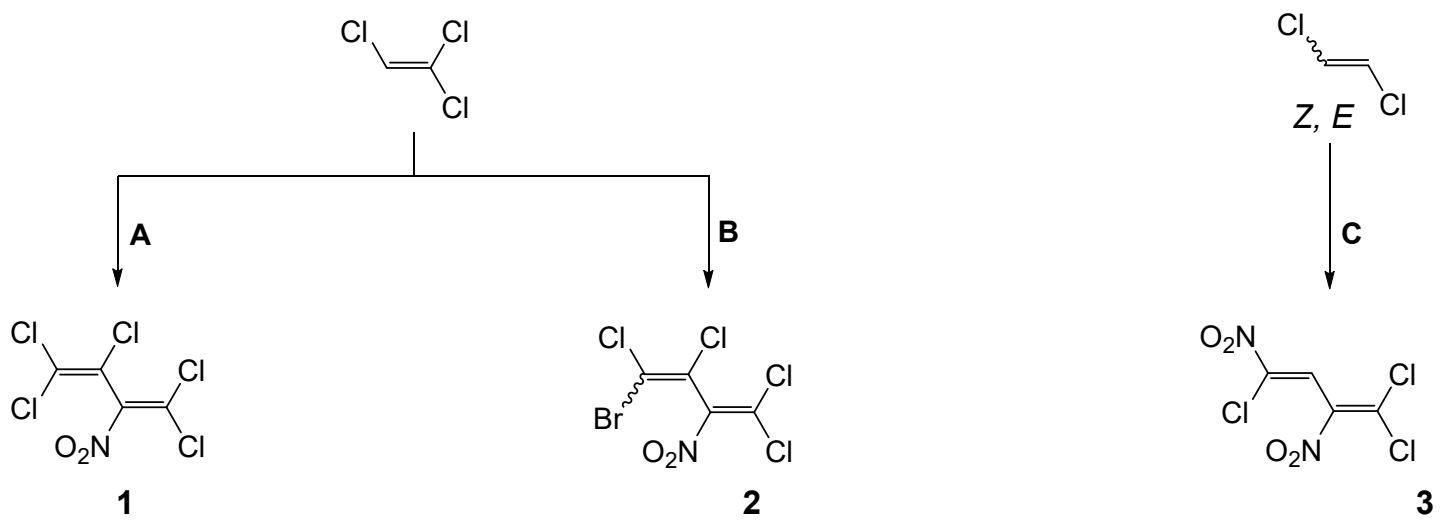

A: i) $\mathrm{BPO}$ ii) $\mathrm{HSO}_{3} \mathrm{Cl}$ iii) $\mathrm{HNO}_{3} / \mathrm{H}_{2} \mathrm{SO}_{4}$.

B: i) $\mathrm{BPO}$ ii) $\mathrm{Zn}$ iii) $\mathrm{Br}_{2}$ iv) $\mathrm{KOH}$ v) $\mathrm{HNO}_{3} / \mathrm{H}_{3} \mathrm{PO}_{4}$.

C: i) $\mathrm{BPO}$ ii) $\mathrm{Br}_{2}$ iii) $\mathrm{KOH}$ iv) $\mathrm{HNO}_{3} / \mathrm{H}_{3} \mathrm{PO}_{4}$.

Scheme 1. Synthesis of the polyhalonitrobutadienes 1-3.

Due to their graded reactivity in $\mathrm{S}_{\mathrm{N}}$ reactions, nitro-substituted polyhalo-1,3-butadienes have proven to be valuable synthetic precursors for a variety of four- to six-membered, often pharmaceutically active heterocycles. They bear a unique substitution pattern that cannot be accessed easily on an alternative pathway. This structural overview for medicinal chemists demonstrates the broad synthetic potential of the nitrodienes $\mathbf{1}, \mathbf{2}$, and $\mathbf{3}$ as a backbone for specifically substituted heterocycles, owing to the additional potential for predictable successive molecular fine tuning.

\section{Results and Discussion}

\subsection{Chemistry}

\subsubsection{Benzoxazolines and Benzimidazolines}

Due to the high electrophilicity of its nitrodichlorovinyl group, $\mathbf{1}$ reacts readily with various amines which, according to Pearson's scale, are hard nucleophiles [7]. Thus, reactions of 2-aminophenol derivatives with 1 occur under mild conditions and lead to substitution of both $\mathrm{Cl}$ groups of the nitrodichlorovinyl unit with formation of the corresponding $(E)$-2,3-di-hydrobenzoxazoles $\mathbf{4 a}, \mathbf{b}$ in reasonable yields [8]. The substructure of a $\beta$-nitro-substituted enamine within compounds $\mathbf{4 a}, \mathbf{b}$ should enable a stabilization caused by a strong hydrogen bond between an oxygen atom of the nitro group and the proton at the oxazoline nitrogen atom. The downfield-shifted ${ }^{1} \mathrm{H} \mathrm{nmr} \mathrm{signal} \mathrm{for} \mathrm{the} \mathrm{NH-group}$ between $11.8 \mathrm{ppm}$ in $\mathrm{CDCl}_{3}$ and $13.6 \mathrm{ppm}$ in DMSO- $d_{6}$ points out that $\mathrm{H}$-bonding (Scheme 2 ) must be important and azoles $\mathbf{4 a}, \mathbf{b}$ are obtained hereby exclusively as $E$-isomers. Under mild reaction conditions, benzoxazoline $4 \mathrm{~b}$ reacts with activated pyridine derivatives, forming betaines $5 \mathrm{a}-\mathrm{c}$ in $62-74 \%$ yield. Treatment of $\mathbf{4 b}$ with nicotine under the same reaction conditions furnishes azinate $\mathbf{5 d}$ in $53 \%$ yield. Synthesis of the novel and structurally interesting cross-conjugated inner salts $\mathbf{5 a - d}$ demonstrates the broad synthetic applicability of 2-nitroperchlorobutadiene [9]. Benzoxazoline 6 was obtained in 73\% yield as a 1:1 mixture of two isomers by the action of 2-amino-3-methylphenol on the bromonitrodiene 2 at $-40{ }^{\circ} \mathrm{C}$ in methanol. The reaction of dinitrodiene 3 with 4 -methylbenzene-1,2-diamine at $-40{ }^{\circ} \mathrm{C}$ results almost quantitatively in the formation of benzimidazoline $7 \mathrm{a}$. By treatment of 3 with 2-aminophenol derivatives, the corresponding benzoxazolines $\mathbf{7 b}, \mathbf{c}$ were obtained in acceptable yields (Scheme 2). Similar benzoxazolines and benzimidazolines exhibit herbicidal activity and act as a model compound when exploring caseinolytic protease as target for herbicides or growth regulators [10]. 
<smiles>[R]c1ccc(O)c(N)c1OC</smiles>

4a, $80 \%, R=H, R^{1}=M e$<smiles>[R]c1ccc2c(c1[R])NC(=C(C(Cl)=C(Cl)Cl)[N+](=O)[O-])O2</smiles>

$4 b, 68 \%, R=M e, R^{1}=H$<smiles></smiles><smiles>CN1CCCC1c1cccnc1</smiles>

4b

5d, $53 \%$<smiles>COc1cccc(O)c1N</smiles>

3<smiles>[R]c1cc([R])c(N)c([R])c1N</smiles><smiles>[R]C1=Cc2c(cc([R])c([R])c2[R])N/C1=C\C(Cl)[N+](=O)[O-]</smiles>

7a, $93 \%, X=N H, R=M e, R^{1}=R^{2}=H$

$7 b, 67 \%, X=O, R=R^{2}=H, R^{1}=M e$

$7 c, 85 \%, X=O, R=R^{1}=H, R^{2}=M e$

Scheme 2. Synthesis of benzoxazolines $4,5,6,7 \mathbf{b}, \mathbf{c}$ and benzimidazoline $7 \mathbf{a}$.

\subsubsection{Imidazolidines}

Imidazolidines $\mathbf{8}$ have been prepared by reaction of nitrodienes $1-3$ with $N^{1}$, $N^{2}$-diphenylethane-1,2-diamine in methanol. Under optimized conditions, yields of the products 8 reached $86-93 \%$ (Scheme 3). Only compound 8a was previously prepared [11] and isolated in $25 \%$ yield by reaction of (1,3,4,4-tetrachloro-2-nitrobuta-1,3-dien-1-yl)( $p$-tolyl)sulfane with $N^{1}, N^{2}$-diphenylethane-1,2-di-amine. Treatment of imidazolidine 8a with a fivefold excess of pyrrolidine in refluxing methanol led to the formation of a $\mathrm{S}_{\mathrm{N}} \mathrm{Vin}$ product, the 2-(3,3-dichloro-1-nitro-2-(pyrrolidin-1-yl)allylidene)-1,3-diphenylimidazolidine (9) in 50\% yield.

\subsubsection{Imidacloprid Analogues}

The imidazolidine Imidacloprid (N-\{1-[(6-chloro-3-pyridyl)methyl]-4,5-dihydroimidazol-2-yl $\}$ -nitramide) has been the most widely used systemic insecticide worldwide. A first synthesis of ana-logues from nitropolychloroalkenes has been reported [12,13], new types of derivatives are presented here. For instance, 11a and $\mathbf{1 1 b}$ were obtained from nitrodiene $\mathbf{1}$ and chloropyridines 10a and 10b, respectively [13]. Reaction of imidazolidine 11a with a 2.5-fold excess of N-nucleophiles such as ethyl piperidine-4-carboxylate and 1,2,3,4-tetrahydroisoquinoline in methanol at $35-50{ }^{\circ} \mathrm{C}$ leads to compounds 12a and 12b in 60-85\% yield, respectively. By using 2-mercaptoethan-1-ol as S-nucleophile for the reaction with 11a in the presence of sodium ethanolate, the corresponding sulfane 
$\mathbf{1 2} \mathrm{c}$ was obtained in $63 \%$ yield. Treatment of $\mathbf{1 1 b}$ with a fivefold excess of dimethylamine at rt led to the formation of oxazolidine $\mathbf{1 2 d}(70 \%)$. By the reaction of bromonitrodiene 2 with an equimolar amount of 4-fluorobenzenethiol in DCM at rt, sulfane 13 was obtained as mixture of two isomers in a total yield of $74 \%$. The subsequent vinylic substitution of the monothio compound $\mathbf{1 3}$ by means of $\mathbf{1 0 a}$ gave imidazolidine 14 (44\%) as well as ketene dithioacetal 15 (30\% yield). Arylthiols are known to be both, good nucleophiles as well as good leaving groups. Compound 14 was previously syn-thesized in $40 \%$ yield directly from nitrodiene 2 and diamine 10a [12]. 1,1-Dithio compound 15 could be obtained in $83 \%$ yield from diene 2 and two equivalents of 4-fluorobenzenethiol using sodium methanolate as a base. The reaction of diene 2 with diamine 16 [13] at optimized conditions furnished the imidazolidine 17 as a mixture of two isomers in a total yield of $89 \%$ (Scheme 4 ).

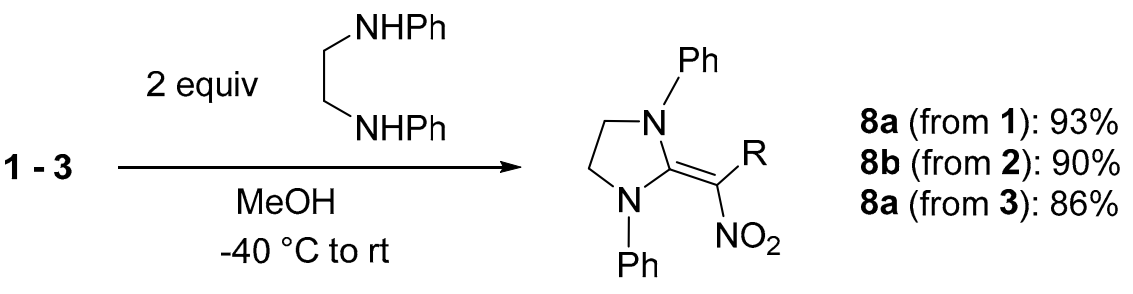

8(a-c)<smiles>O=[N+]([O-])C(C(Cl)=C(Cl)Cl)=C1N(c2ccccc2)CCN1c1ccccc1</smiles>

$8 a$<smiles>O=[N+]([O-])C(C(=C(Cl)Cl)N1CCCC1)=C1N(c2ccccc2)CCN1c1ccccc1</smiles>

9, 50\%

Scheme 3. Synthesis of imidazolidines 8,9 .

\subsubsection{Thiazolidinones}

Thiazolidin-4-ones represent a class of compounds that has proven to exhibit distinctive bio-activity, e.g. antifungal, antibacterial, antitubercular, and anticonvulsant properties [14-17]. Our research in this area is presented through an efficient synthesis of functionalized (Z)-2-allylidene-thiazolidin-4-ones [18]. Nitrodiene 1 reacts with ethyl 2-mercaptoacetate to give the sulfane $\mathbf{1 8}$ as single E-isomer [19]. For the subsequent reactions of sulfane 18, we used two aniline derivatives, an activated (ERG) and an desactivated (EWG) one. In both cases, the expected thiazolidinones 19a,b were obtained in good yields (73-76\%). Treatment of $\mathbf{1 9} \mathbf{a}, \mathbf{b}$ with a 5 - to 8-fold excess of hydrazine led to pyrazoles $20 \mathbf{a}, \mathbf{b}$. The assumed mechanism for this ring-opening and subsequent ring-closure transformation forming 20a,b has been presented [18]. Heating of thiazolidinones 19a,b with five membered 2-formyl heterocycles in acetic acid in the presence of trimethylamine furnished hetarylmethylidenethiazolidinones 21a,f in good to excellent yields, as single diastereomers. The Z-configuration was assigned according to literature data. The presence of only one signal for the methylidene proton at 7.72-8.15 ppm in the ${ }^{1} \mathrm{H}$ nmr spectra of compounds 21a,f suggested the formation of a single isomer, which was assigned to the Z-configuration according to the literature for similar compounds [20,21]. Interestingly, close analogues of these structures, i.e., the 5-arylmethylidene rhodanines, possess photosynthesis-inhibiting and antialgae properties [22], show anticancer activity [23,24], and are inhibitors of bacterial enzyme synthetase MurD with E. coli [25]. 
<smiles>[Y]CCNCc1ccc(Cl)nc1</smiles>

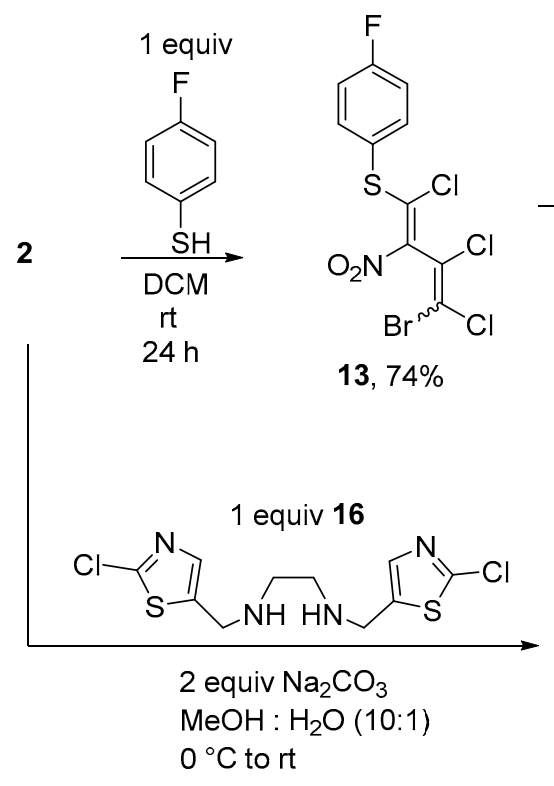

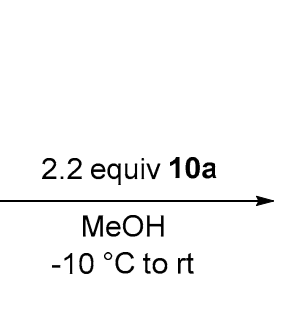

13, $74 \%$

Scheme 4. Synthesis of Imidacloprid analogues 11, 12, 14, 17.<smiles>O=[N+]([O-])C(=C(Cl)C(=[18O])Cl)C1=C(Cc2cnc(Cl)s2)N(Cc2cnc(Cl)s2)CC1</smiles><smiles>CCC</smiles><smiles>O=[N+]([O-])C1=C(C(Cl)C(Cl)Br)N(Cc2ccc(Cl)nc2)CCN1</smiles>

$14,44 \%$

$15,30 \%$

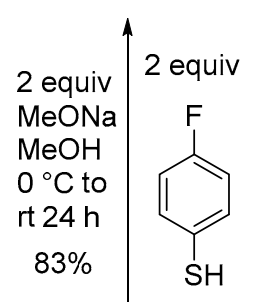

2<smiles>c1ccccc1</smiles> 
<smiles>CCOC(=O)CSC(Cl)=C(C(Cl)=C(Cl)Cl)[N+](=O)[O-]</smiles>

18, $80 \%$ from 1

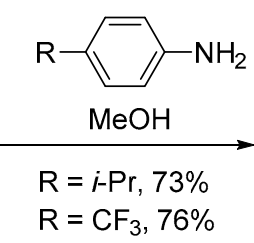

$\mathrm{CF}_{3}, 76 \%$<smiles>[R]c1ccc(N2C(=O)CS/C2=C(\C(Cl)=C(Cl)Cl)[N+](=O)[O-])cc1</smiles>

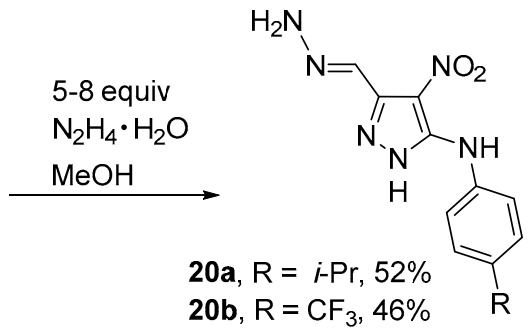<smiles>[X]c1ccc(/C=c2\s/c(=C(\C(Cl)=C(Cl)Cl)[N+](=O)[O-])n(-c3ccc([R])cc3)c2=O)cc1</smiles>

21(a-f)

21a, $\mathrm{R}=i-\operatorname{Pr}, \mathrm{X}=\mathrm{O}, 96 \%$

21b, $\mathrm{R}=i-\operatorname{Pr}, \mathrm{X}=\mathrm{S}, 92 \%$

21c, $\mathrm{R}=i-\mathrm{Pr}, \mathrm{X}=\mathrm{NMe}, 92 \%$

21d, $\mathrm{R}=\mathrm{CF}_{3}, \mathrm{X}=\mathrm{O}, 73 \%$

21e, $\mathrm{R}=\mathrm{CF}_{3}, \mathrm{X}=\mathrm{S}, 85 \%$

21f, $\mathrm{R}=\mathrm{CF}_{3}, \mathrm{X}=\mathrm{NMe}, 55 \%$

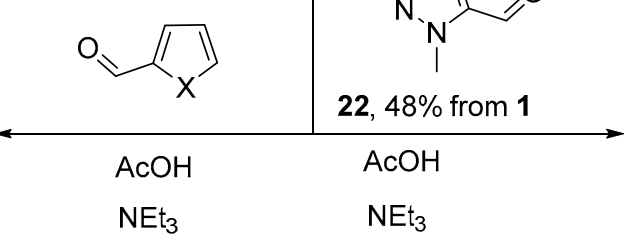

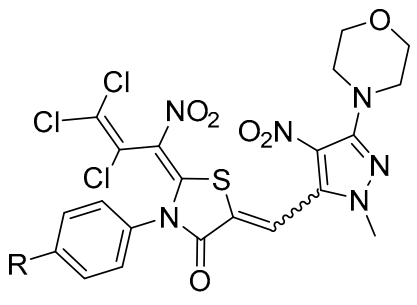

23(a-b), 2 isomers, ca. 86: 14

23a, $\mathrm{R}=i-\mathrm{Pr}, 94 \%$

23b, $\mathrm{R}=\mathrm{CF}_{3}, 97 \%$

Scheme 5. Synthesis of thiazolidinones 19, 21, 23 and pyrazoles 20 and 23.

4-Ethoxy-2-(2,3,3-trichloro-1-nitro-2-propenylidene)-benzazetine (a regioisomer of compound 26b) can modulate RNA binding proteins [31]. A new prenylated indole alkaloid taichunamide A contains a benzazetidine unit, too [32]. It was isolated from the fungus Aspergillus taichungensis.

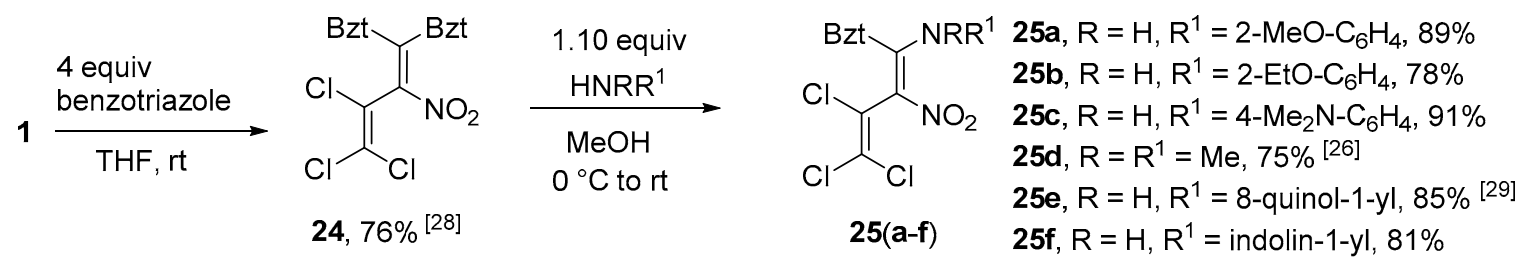

25(a-c) $\underset{\text { reflux }}{\stackrel{\text { solvent }}{\longrightarrow}}$<smiles>[R]c1cc([R])c2c(c1)/C(=C(\C(Cl)=C(Cl)Cl)[N+](=O)[O-])N2</smiles>

26a, $\mathrm{R}^{2}=\mathrm{H}, \mathrm{R}^{3}=\mathrm{MeO}$, solvent $-\mathrm{MeOH}, 37 \%$

26b, $R^{2}=H, R^{3}=E t O$, solvent - EtOH, $27 \%$

26c, $R^{2}=\mathrm{Me}_{2} \mathrm{~N}, \mathrm{R}^{3}=\mathrm{H}$, solvent $-\mathrm{THF}, 25 \%$

Scheme 6. Synthesis of benzazetines 26 and benzotriazoles 25 .

\subsubsection{Pyrimidines}

In the course of the studies concerning polyhalogenated nitrobutadienes, a new ring closure approach to perfunctionalized 5-nitropyrimidines was also developed [33]. Using this protocol starting from $\mathbf{2 5} \mathbf{c}-\mathbf{f}$, four new nitropyrimidines $\mathbf{2 7} \mathbf{c}-\mathbf{f}$ were obtained. Even under optimum conditions, yields of the products $27 \mathrm{c}-\mathbf{f}$ remained moderate, reaching $49-65 \%$. The assumed mechanism for the formation of pyrimidines 27 has been presented in the literature [33]. 5-Nitro-substituted pyrimidines are interesting precursors for the synthesis of a wide range of poly-substituted pyrimidines and other heterocyclic systems with potential biological activity [34]. Among numerous applications, some examples are noteworthy: cytotoxic activity is documented $[35,36]$ as well as the potential to inactivate the human DNA repair process [37]. The broad variety of medicinal applications is further illustrated, e.g. with the 
activity against chronic obstructive pulmonary disease [38], applicability against herpes simplex [39], and other viral diseases [40]. Furthermore, one field of application of 5-nitropyrimidines uses their positive modulating effect of the GABAB receptor [41,42]. Pyrimidin-4-yl- $1 \mathrm{H}$-indoles are a very rare class of organic compounds; to the best of our knowledge, only 4 representatives are known till today [43-45]. With the aim to synthesize a new pyrimidin-4-yl- $1 H$-indole with potent biological activity, we made an attempt to oxidize the 2,3-dihydroindole 27f. Indeed, by using DDQ as oxidizing agent (ratio 27f: DDQ 1: 2.25, toluene, reflux $5 \mathrm{~h}$ ), the expected indole 28 was obtained in $66 \%$ yield (Scheme 7).<smiles>[R17]NC(=[Pt])C(=C(Cl)Cl)[N+](=O)[O-]</smiles>

25(c-f)

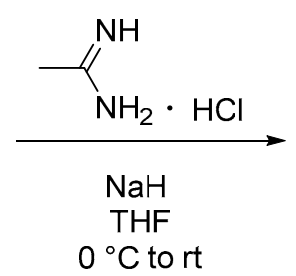

27c, $\mathrm{R}=\mathrm{H}, \mathrm{R}^{1}=4-\mathrm{NMe}_{2}-\mathrm{C}_{6} \mathrm{H}_{4}, 65 \%$

27d, $R=R^{1}=M e, 56 \%$

27e, $R=H, R^{1}=8$-quinol-1-yl, $49 \%$

27f, $N_{R R}{ }^{1}=$ indolin-1-yl, $64 \%$

27(c-f)<smiles>Cc1nc(C(Cl)Cl)c([N+](=O)[O-])c(N2CCc3ccccc32)n1</smiles>

$27 f$

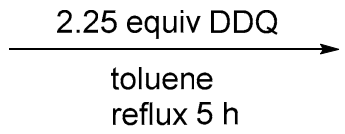

reflux $5 \mathrm{~h}$<smiles>Cc1nc(C(Cl)Cl)c([N+](=O)[O-])c(-n2ccc3ccccc32)n1</smiles>

$28,66 \%$

Scheme 7. Synthesis of pyrimidines 27, 28.

\subsubsection{Pyrazoles}

In the past ten years, different ways to synthesize persubstituted 4-nitropyrazoles have been developed $[9,18,26,28,46]$. Some of these compounds show high biological activities: they can modulate the biological activity of IFNs-I [47], are active against mycobacterial infections including tuberculosis [48], and are able to reduce prime virulence factors of Vibrio cholerae [49,50]. In the course of our recent studies, the three 1-amino-1-benzotriazolyldienes $25 \mathrm{~g}-\mathbf{i}$ were synthesized in good yields (85-91\%) from bis(benzotriazole) 24 using 4-(4-chlorophenyl)piperidin-4-ol, piperazin-1-yl-(tetrahydrofuran-2-yl)methanone and 1-(3-chlorophenyl)piperazine, respectively. The

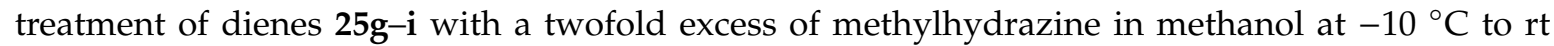
led to the formation of new nitropyrazoles $29 \mathrm{a}-\mathrm{c}$ in good yields $(74-79 \%)$. By saponification of the dichloromethyl group in compound $29 \mathrm{c}$ by means of $25 \%$ aqueous sulfuric acid at $95-100{ }^{\circ} \mathrm{C}$, the aldehyde 30 was obtained (57\%) (Scheme 8) [26]. Other 4-nitropyrazoles are known as hypoxia-selective cytotoxins and radiosensitizers [51]; they can be useful as herbicides [52,53] or show psychosedative actions [54].

\subsubsection{H-Pyrido $[1,2-a]$ Pyrimidines}

Recently, a new pathway for the synthesis of 4-(dichloromethylene)-3-nitro-4H-pyrido[1,2-a] pyrimidines with a unique substitution pattern at the pyrimidine ring was developed [28]. Starting with bis(benzotriazole) 24, using a threefold excess of 2-aminopyridines two enamines $\mathbf{3 1 a}, \mathbf{b}$ were formed in THF in $81-86 \%$ yield. The benzotriazole unit in pyridopyrimidines 31 , activated through the neighboring nitro group, acts as a very good leaving group and can be replaced with different nucleophiles at mild reaction conditions. Thus, treatment of compounds 31 with 1-(4-fluoro-phenyl)piperazine in methanol at $40{ }^{\circ} \mathrm{C}$ led to the piperazino-substituted pyridopyrimidines 32 in excellent yields. A $S_{N}$ Vin process in pyridopyrimidines 31 under action of S-nucleophiles such as ethyl 2-mercaptoacetate using sodium ethanolate as base furnished sulfane $33 \mathbf{a}, \mathbf{b}$ in $93-97 \%$ yield. Another possibility to form compounds $\mathbf{3 3} \mathbf{a}, \mathbf{b}$ is reaction of sulfane $\mathbf{1 8}$ with 2 -aminopyridines. At optimum conditions $(\mathrm{MeOH}$, threefold 
excess of amino-pyridines, rt), the yields of pyridopyrimidines 33 reached $47-55 \%$ (Scheme 9). Similar pyrido[1,2-a]pyrimidines show antiviral [55], antithrombotic [56], and antibacterial [57-60] activities.

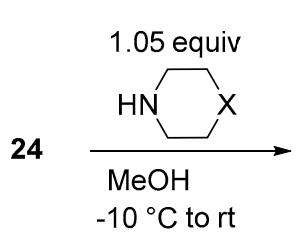

$\mathrm{X}=\mathrm{C}(\mathrm{OH})-\mathrm{C}_{6} \mathrm{H}_{4}-4-\mathrm{Cl}$<smiles>[X]=NC(=O)C1CCCO1</smiles><smiles>[X]=Nc1cccc(Cl)c1</smiles><smiles>O=[N+]([O-])C(=C(Cl)C(Cl)Cl)C1CCCCC1</smiles>

25g, $91 \%$

$25 \mathrm{~h}, 85 \%$

$25 \mathbf{i}, 88 \%$

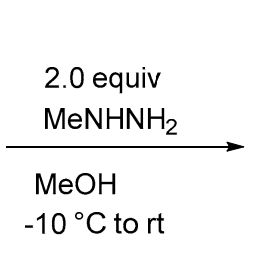<smiles>Cn1nc(N2CCCCC2)c([N+](=O)[O-])c1C(Cl)Cl</smiles>

29a, $74 \%$

29b, $79 \%$

$29 c, 74 \%$

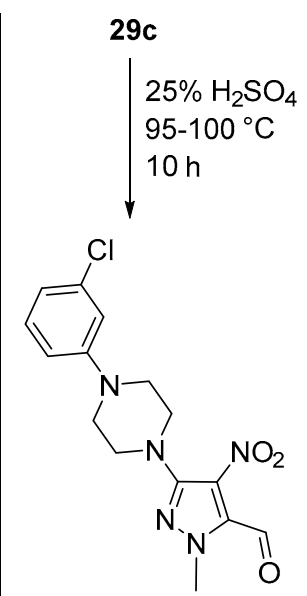

$30,57 \%$

Scheme 8. Synthesis of pyrazoles 29, 30 and benzotriazoles 25 .<smiles>[R]c1ccc(N)nc1</smiles><smiles>CCOC(=O)CS/C(Cl)=C(/C(Cl)=C(Cl)Cl)[N+](=O)[O-]</smiles><smiles>[R]C1=CN2C(=NC(n3nnc4ccccc43)=C([N+](=O)[O-])C2=C(Cl)Cl)C=C1</smiles><smiles>COCOOC</smiles><smiles>[R]C1=CN2C(=NC(N3CCN(c4ccc(F)cc4)CC3)=C([N+](=O)[OH2+])C2=C(Cl)Cl)C=C1[R6]#[R16]</smiles>

31a, $\mathrm{R}=\mathrm{H}, 81 \%$ 31b, $\mathrm{R}=\mathrm{Cl}, 86 \%$

$32 \mathrm{a}, \mathrm{R}=\mathrm{H}, 88 \%$
32b, R $=\mathrm{Cl}, 95 \%$
2 equiv $\mathrm{HSCH}_{2} \mathrm{CO}_{2} \mathrm{Et}$

3 equiv $\mathrm{NaOEt}$, EtOH, rt

33a, $\mathrm{R}=\mathrm{H}, 97 \%$

33b, $\mathrm{R}=\mathrm{Cl}, 93 \%$

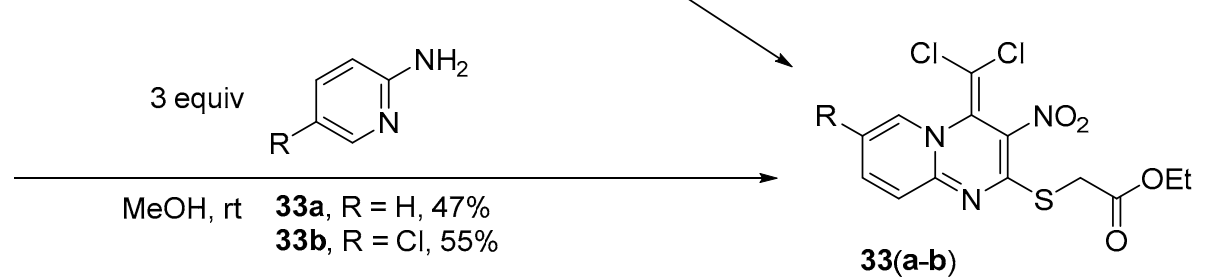

Scheme 9. Synthesis of 4H-pyrido[1,2-a]pyrimidines 31-33.

\subsubsection{Benzo[h]quinolines}

In the course of our studies on polyhalogenated nitrobutadienes, a new ring closure approach to benzo[h]quinolines was also developed [61]. Starting from nitrodiene $\mathbf{1}$ in three steps, the target benzo[ $h]$ quinolines with a unique substitution pattern at the pyridine ring were obtained in good yields. In detail, after mono substitution of one chlorine group in diene $\mathbf{1}$ sulfanes $\mathbf{3 4 a} \mathbf{a}, \mathbf{b}$ were formed as single isomers each in yields of about $80 \%$ according to the literature [62] for the benzyl derivative $34 \mathbf{a}$ and literature [9] for the 4-chlorophenyl derivative $34 \mathbf{b}$. In a second step, we synthesized the aminothiobutadienes $\mathbf{3 5 a}, \mathbf{b}$ by interaction of sulfane $\mathbf{3 4} \mathbf{a}, \mathbf{b}$ with an excess of 1 -naphthylamine in methanol at $-10{ }^{\circ} \mathrm{C}$ to rt. Dienes $35 \mathbf{a}, \mathbf{b}$ were also formed (76-85\% yield) as single $E$-isomers due to the stable six membered hydrogen bridge between the amino and nitro group. Finally, the ring closure at optimum conditions (twofold excess of triethylamine as a base) proceeded under formation of the expected benzo[ $h$ ]quinolines $\mathbf{3 6 a}, \mathbf{b}$ in good yields (76-85\%). The assumed mechanism for the formation 
of benzo[h]quinolines is depicted in the literature [61]. Benzo[h]quinolines are a precious class of organic compounds and show interesting biological properties [63-67]. Oxidation of quinolines $\mathbf{3 6 a}, \mathbf{b}$ with excess of hydrogen peroxide in a mixture of acetic acid and chloroform lead to the formation of sulfoxides $\mathbf{3 7 a} \mathbf{a}, \mathbf{b}$ in $89-91 \%$ yield. The sulfinyl group is known to be a good leaving group [68-70]. Indeed, treatment of sulfoxide $37 \mathrm{a}$ with an excess of pyrrolidine in toluene at $100{ }^{\circ} \mathrm{C}$ furnished amino derivative 38 in $77 \%$ yield (Scheme 10).
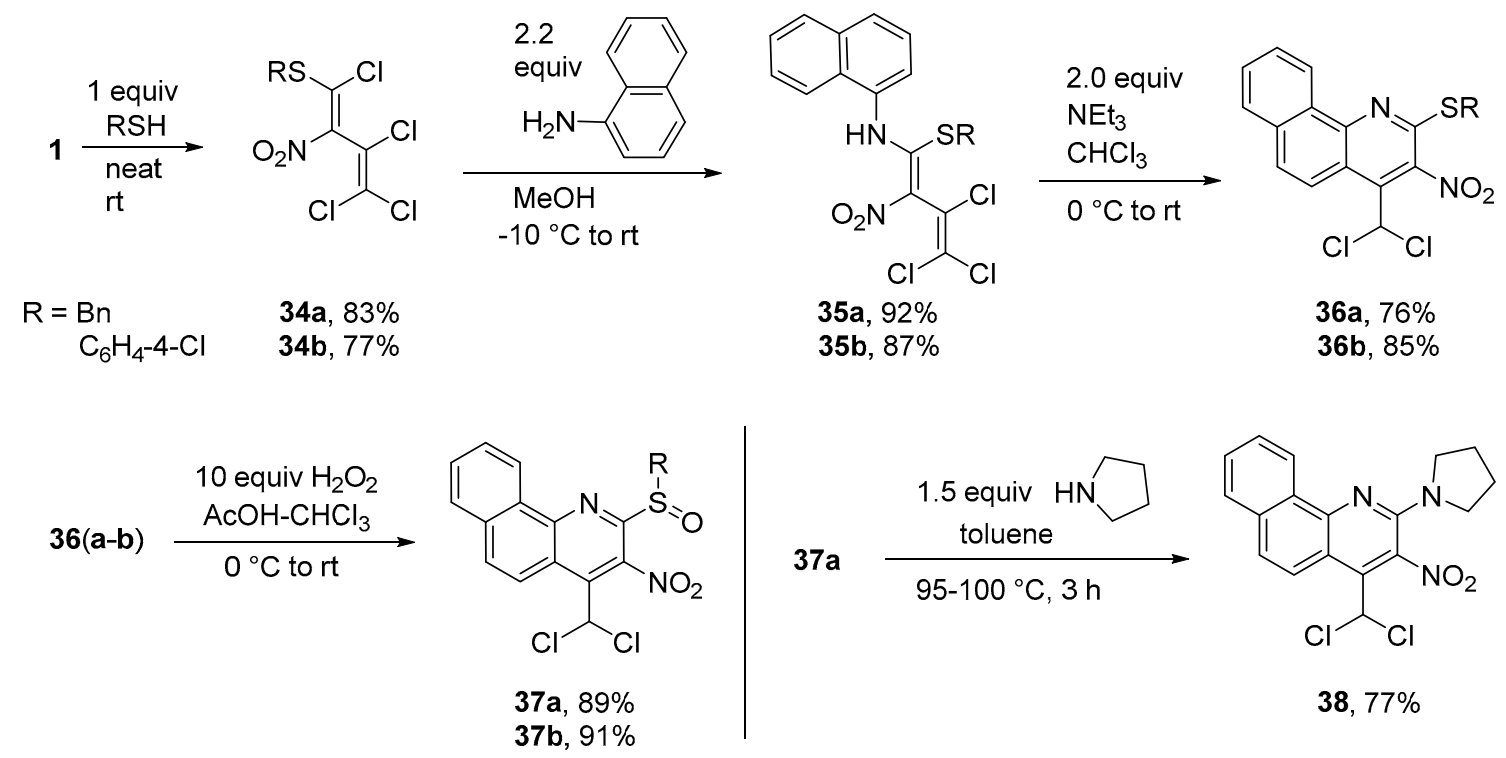

Scheme 10. Synthesis of benzo[h]quinolines $\mathbf{3 6 - 3 8}$ and nitrodienes $\mathbf{3 4}, \mathbf{3 5}$.

\subsubsection{Isothiazoles}

The isothiazole 39 was obtained from nitrodiene 1 upon treatment with elemental sulfur at $200{ }^{\circ} \mathrm{C}$ [71]. Subsequent reaction with fuming nitric acid provided the 4,5-dichloroisothiazole-3-carboxylic acid (40) [72], which could be easily converted into the corresponding acid chloride $\mathbf{4 2}$ with thionyl chloride (93\% yield) [28]. Acid $\mathbf{4 0}$ reacts with ethyl 2-bromoacetate in the presence of sodium ethanolate under reflux conditions to ester 41 in $58 \%$ yield. The esterification of acid chloride 42 with a fourfold excess of 2,2,2-trifluoroethan-1-ol in refluxing THF resulted in the formation of a 2,2,2-trifluoroethyl 4,5-dichloroisothiazole-3-carboxylate (43) (64\% yield). Acid chloride 42 smoothly reacted with aromatic and aliphatic amines to provide the corresponding amides $44 a-d$ in $68-95 \%$ yield. The reaction of chloride 42 with 2 equivalents of 1-(6-chloropyridin-3-yl)- $\mathrm{N}$-methyl-methanamine in THF furnished amide 44e as mixture of two rotamers in relation 10: 6 with a total yield of $86 \%$. An alternative way to obtain amide 44e is the interaction of carbohydrazide 44a with 1-(6-chloropyridin-3-yl)- $N$-methylmethanamine at harder reactions conditions (DMSO, $95-100{ }^{\circ} \mathrm{C}$ ). In this case, amide 44e is formed as a mixture of two rotamers (relation 10:6), with a total yield of 69\%. Reacting hydrazide 44a with a fourfold excess of morpholine (DMSO, 90-95 ${ }^{\circ} \mathrm{C}$ ) did not lead to the formation of a product similar to amide 44e. Instead, upon substitution of a chlorine group in 5 position of the heterocyclic ring, an isothiazole 45 was formed (78\% yield). These amides $44 a-\mathbf{a}$ and 45 are interesting candidates for biological testing, as amides of 4-chloroisothiazol-3-carboxylic acid have been shown to exhibit high antibacterial activity [73-75]. Finally, we investigated the interaction of amides $44 a-d$ and ester 41 with S-nucleophiles such as 4-chlorobenzenethiol. In all cases, reaction products at 5 position of the heterocyclic ring were formed. Under optimized reaction conditions, the yields of the 5-((4-chlorophenyl)thio)isothiazoles 46a-e were in the range of $67-89 \%$ (Scheme 11). 
<smiles>Clc1snc(C(Cl)(Cl)Cl)c1Cl</smiles>

$39,55 \%$ from 1

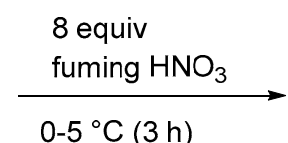

to $10-15^{\circ} \mathrm{C}, 1 \mathrm{~d}$<smiles>O=C(O)c1nsc(Cl)c1Cl</smiles>

40, $90 \%$

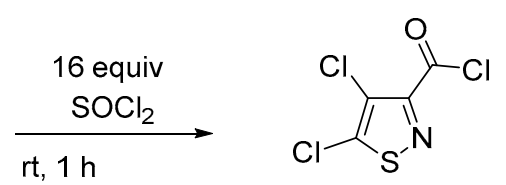

42, $93 \%$

to reflux, $4 \mathrm{~h}$
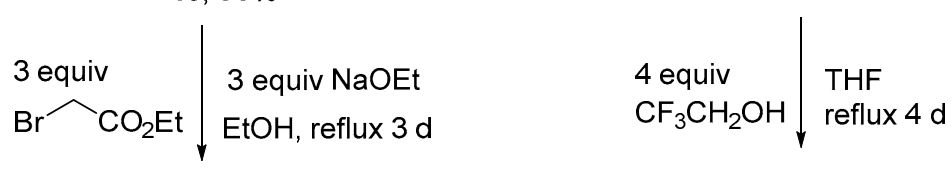<smiles>CCOC(=O)COC(=O)c1nsc(Cl)c1Cl</smiles>

$41,58 \%$<smiles>O=C(OCC(F)(F)F)c1nsc(Cl)c1Cl</smiles>

43, $64 \%$
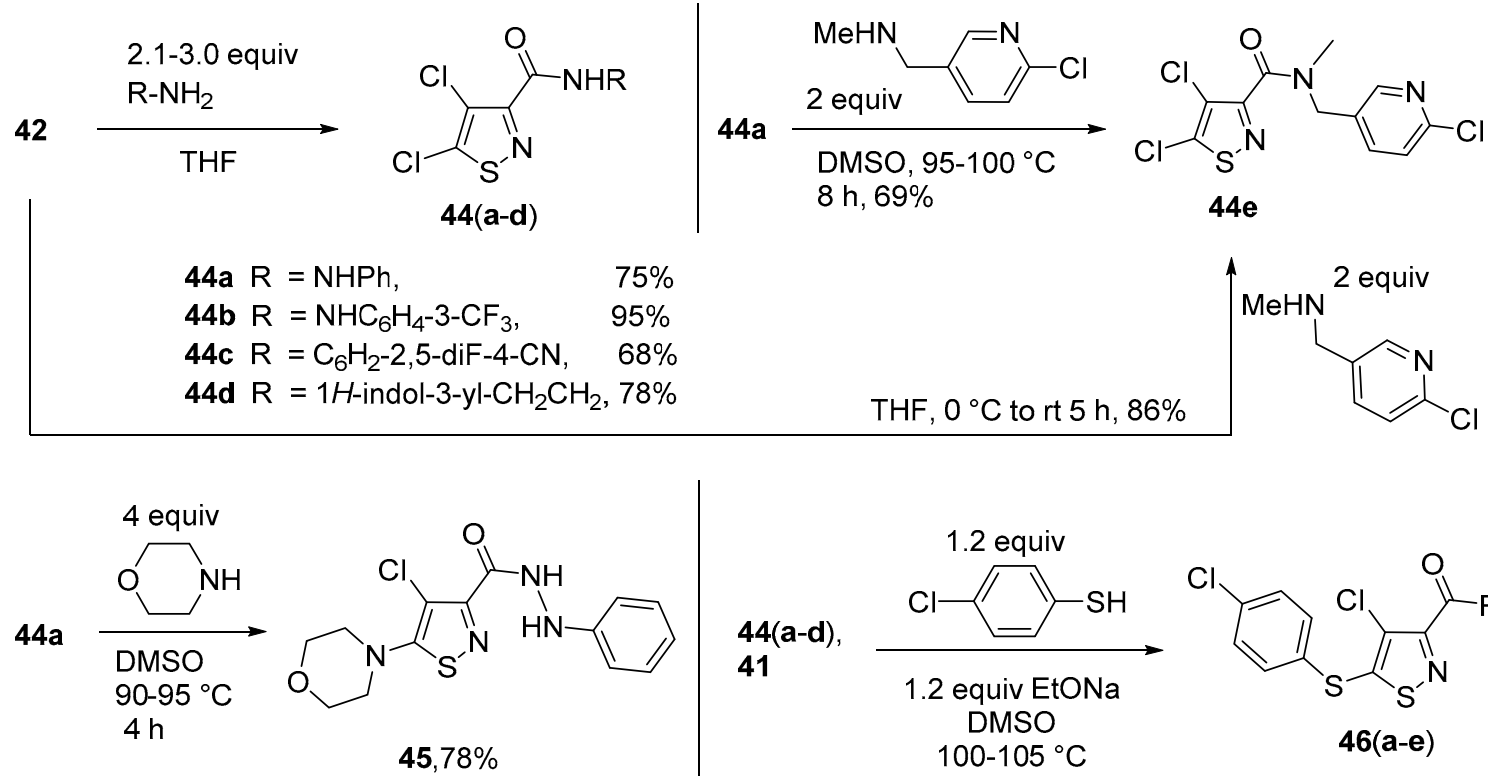

44(a-d) 41<smiles>[R]C(=O)c1nsc(Sc2ccc(Cl)cc2)c1Cl</smiles>

46(a-e)<smiles>CNCc1ccc(Cl)nc1</smiles>
46a $\mathrm{R}=\mathrm{NHNHPh}$,
$67 \%$
46b $\mathrm{R}=\mathrm{NHNHC}_{6} \mathrm{H}_{4}-3-\mathrm{CF}_{3}, \quad 70 \%$
46c $\mathrm{R}=\mathrm{NHC}_{6} \mathrm{H}_{2}-2,5-\mathrm{diF}-4-\mathrm{CN}, \quad 83 \%$
46d $\mathrm{R}=1 \mathrm{H}$-indol-3-yl- $\mathrm{CH}_{2} \mathrm{CH}_{2} \mathrm{NH}, 89 \%$
46e $\mathrm{R}=\mathrm{OCH}_{2} \mathrm{CO}_{2} \mathrm{Et}$,
$68 \%$

Scheme 11. Synthesis of isothiazoles 39-46.

\subsubsection{4,5-Dihydroisoxazoles}

Recently, we developed a novel, fairly general method for the synthesis of dihydroisoxazoles with a chlorinated side chain in 3-position, starting from gem-dichloronitroalkenes [76]. In particular, reaction of nitrodiene 1 with a base leads to the formation of 2,3,3-trichloroprop-2-enenitrile oxide. Trapping of this nitrile oxide with an alkene resulted in 4,5-dihydroisoxazole derivatives 47-50, due to a 1,3-dipolar cycloaddition. In detail, reaction of nitrodiene $\mathbf{1}$ with prop-1-en-2-ylbenzene at optimized conditions furnished 4,5-dihydroisoxazole 47 in $76 \%$ yield. The interaction of the acrylonitrile oxide $\mathbf{1}$ with tert-butyl acrylate and 2-ethylhexyl acrylate at similar reactions conditions provided alkyl 3-(1,2,2-trichlorovinyl)-4,5-dihydroisoxazole-5-carboxylates 48 and 49 (65 and 60\% yield). Upon treatment of diene $\mathbf{1}$ with cyclohexene, the hexahydrobenzo- $[d]$ isoxazole $\mathbf{5 0}$ was isolated in $41 \%$ yield (Scheme 12). The yields of $\mathbf{4 7 - 4 9}$ are quite similar to certain compounds in [76], whereas for $\mathbf{5 0}$ (in 
analogy to the reaction with cyclopentene [76]), the yield is lower as expected. 4,5-Dihydroisoxazoles were found to exhibit potent cytotoxic, antineoplastic [77], and antimalarial activity [78]. Additionally, they can be used as androgenic or antiandrogenic agents or androgen receptor modulators [79] and show antibacterial and antifungal activities [80].<smiles>OCC(Cl)=C(Cl)Cl</smiles>

from 1

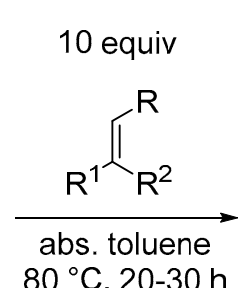

$80^{\circ} \mathrm{C}, 20-30 \mathrm{~h}$

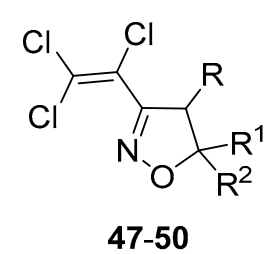

47, $\mathrm{R}=\mathrm{H}, \mathrm{R}^{1}=\mathrm{Me}, \mathrm{R}^{2}=\mathrm{Ph}, 76 \%$

48, $\mathrm{R}=\mathrm{R}^{1}=\mathrm{H}, \mathrm{R}^{2}=\mathrm{CO}_{2} \mathrm{Bu}-t, 65 \%$

49, $\mathrm{R}=\mathrm{R}^{1}=\mathrm{H}, \mathrm{R}^{2}=\mathrm{CO}_{2} \mathrm{CH}_{2} \mathrm{CH}(\mathrm{Et}) \mathrm{Bu}, 60 \%$

50, $\mathrm{R}-\mathrm{R}^{2}=\left(\mathrm{CH}_{2}\right)_{4}, 41 \%$

Scheme 12. Synthesis of 4,5-dihydroisoxazoles 47-50.

\subsubsection{Thiophenes}

In the course of studying nitroperchlorobutadiene $\mathbf{1}$ as a versatile building block for the directed synthesis of a range of persubstituted heterocycles, we also developed a three-step synthesis to persubstituted 3-amino-4-nitrothiophenes [81]. Incorporating both, an enamine and a thioketene unit, these thiophenes are very electron-rich heterocycles with a unique substitution pattern. Starting from 1, the piperazine derivative $\mathbf{5 2}$ was obtained in $90 \%$ yield via the dithiolane $\mathbf{5 1}$. The push-pull substituted thiophene 53 was efficiently accessible in $85 \%$ yield upon treatment of dithiolane 52 with sodium hydroxide using DMSO as solvent. The regioselective ipso-formylation of the 2-chloro-thiophene 53 under Vilsmeier-Haack conditions led to the carbaldehyde 54 (64\% yield), according to [82].

The next successive steps were performed with the most stable derivative 3-morpholino-4-nitro-5-(vinylthio)thiophene-2-carbaldehyde (55) [82]. Knoevenagel condensation of thiophene 55 with malononitrile in ethanol in the presence of a catalytic amount of sodium ethanolate gave the gem-dicyanovinylthiophene 56 in $68 \%$ yield [83]. Oxidation of the vinylsulfanylthiophene 56 with threefold excess of hydrogen peroxide in acetic acid at $50-55{ }^{\circ} \mathrm{C}$ furnished sulfone 57 in $82 \%$ yield. Interaction of carbaldehyde 55 with an excess of dimedone, (2,4,6-trichlorophenyl)hydrazine and Meldrum's acid in methanol led to the formation of bis(5,5-dimethylcyclohexane-1,3-dione) 58, vinylsulfanyl-thiophene 59 and 1,3-dioxane-4,6-dione 60, respectively (Scheme 13).

2-Chloro-3-amino-4-nitro-5-(vinylsulfanyl)thiophenes similarly to compound $\mathbf{5 3}$ were identified as anti-HIV compounds to treat drug-resistant retroviral infections [84] and show antiviral activity [85]. Other 3(4)-nitrothiophenes can be used in fungicidal and/or bactericidal compositions [86], and show insecticidal and acaricidal activity [87].

\subsection{Biological Activity of the Polyhalonitrobutadiene Derivatives}

Evaluation of the biological activity of the chosen polyhalonitrobutadiene derivatives showed that most of them did not display antibacterial or cytotoxic effects, i.e., residual growth or viability after incubation for 1 and 3 days, respectively, were higher than $50 \%$. Tables with all primary screening data are shown in Supplementary Figures S204-S205. None of the derivatives showed an antibacterial activity against the uropathogenic Escherichis coli strain UPEC 796, whereas some had antibacterial activity against Staphylococcus aureus. The cytotoxic activity of nine compounds could be proven, as these compounds had $\mathrm{IC}_{50}$-values $<50 \mu \mathrm{M}$ in the viability assay. Among those compounds was the "conjugate" $\mathbf{2 3 b}$ of the pyrazole 22 and the thiazolidinone $\mathbf{1 9 b}$. Whereas the compound series 21 was more or less completely inactive, introduction of the pyrazole group proved successful. In particular, the introduction of a $\mathrm{CF}_{3}$ substituent resulted in a compound with significant cytotoxicity $\left(\mathrm{IC}_{50}=6.2 \pm 1.8 \mu \mathrm{M}\right)$. Similarly, among the pyrimidines 27,28 , the most potent derivatives were those with the aromatic residues at the nitropyrimidine-core, namely $27 \mathrm{c}$ and 28 with $\mathrm{IC}_{50}$-values of $1.5 \pm$ $0.4 \mu \mathrm{M}$ and $1.05 \pm 0.2 \mu \mathrm{M}$, respectively. The non-aromatic nature of the ring next to the pyrimidine core in $\mathbf{2 7 f}$ prevented the cytotoxic activity. Following the synthesis route of the pyridopyrimidines 
32 and 33 revealed that the precursor with the leaving group benzotriazole was the only cytotoxic compound $\left(\mathrm{IC}_{50}=6.0\right.$ and $\left.5.7 \pm 1.0 \mu \mathrm{M}\right)$, and that cytotoxicity was lost, when the benzotriazole group was replaced. In addition, the benzo[h]quinolines 36, 38 lost the cytotoxic activity, which was still observed for the intermediate naphthalene-aminothiobutadiene 35. All tested derivatives of both the groups of isothiazoles and dihydroisoxazoles were inactive, whereas among the thiophenes, the derivatives with a cyclic dione residue 58 and 60 represented cytotoxic compounds $\left(\mathrm{IC}_{50}=3.1 \pm 0.4\right.$ $\mu \mathrm{M}$ and $20.1 \pm 3.9 \mu \mathrm{M})$. Obviously, the morpholino-nitrothiophene structure was not sufficient for biological activity as compound 59 was completely inactive.
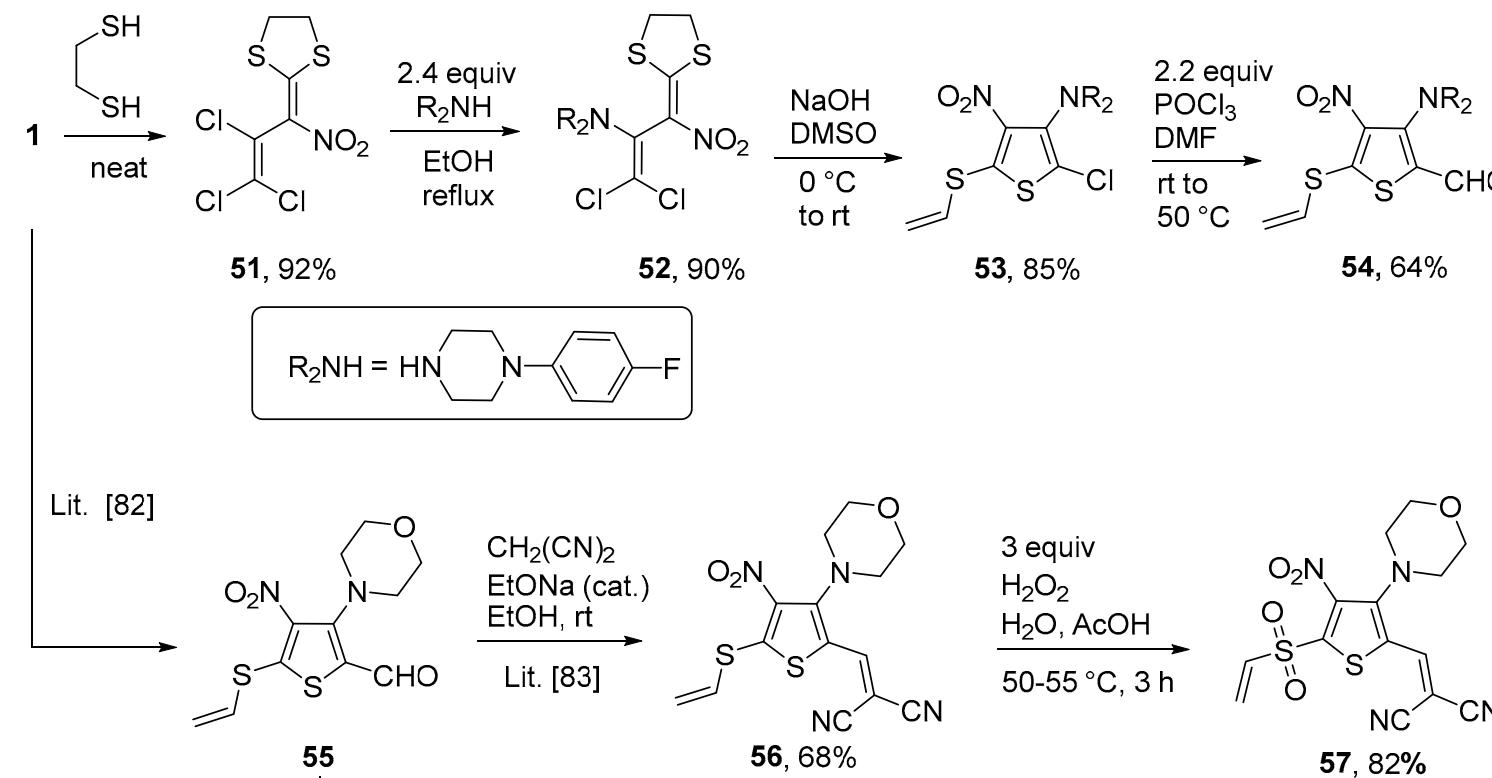

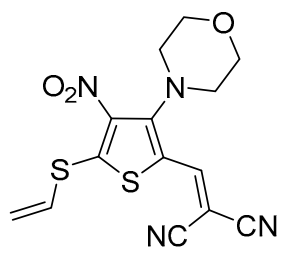

$56,68 \%$

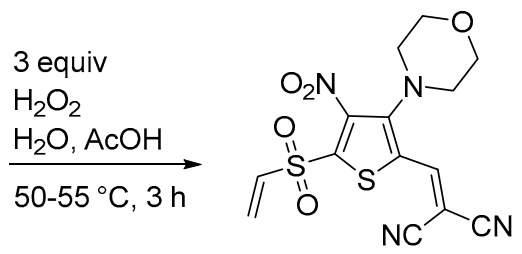

$57,82 \%$<smiles>C=CSc1sc(C(C2C(=O)CC(C)(C)CC2=O)C2C(=O)CC(C)(C)CC2=O)c(N2CCOCC2)c1[N+](=O)[O-]</smiles>

$58,73 \%$<smiles>C=CSc1sc(/C=N/Nc2c(Cl)cc(Cl)cc2Cl)c(N2CCOCC2)c1[N+](=O)[O-]</smiles>

$59,86 \%$<smiles>C=CSc1sc(C=C2C(=O)OC(C)(C)OC2=O)c(N2CCOCC2)c1[N+](=O)[O-]</smiles>

$60,78 \%$

i) 2.2 equiv dimedone, pyridine (cat.), $\mathrm{MeOH}$, rt to $35-40^{\circ} \mathrm{C}, 2 \mathrm{~d}$

ii) 3.0 equiv (2,4,6-trichlorophenyl)hydrazine, $\mathrm{MeOH}$, reflux $3 \mathrm{~h}$

iii) 2.0 equiv Meldrum's acid, piperidine (cat.), $\mathrm{MeOH}, \mathrm{rt}, 3 \mathrm{~d}$

Scheme 13. Synthesis of thiophenes 53-60 and dithiolanes 51, 52.

\section{Experimental}

\subsection{General Information}

General Remarks: Solvents and reagents were used as received from commercial sources without further purification. TLC was performed with Merck aluminum-backed TLC plates with silica gel 60, F254. Flash column chromatography was performed with Macherey-Nagel silica gel 60 M (0.040-0.063 $\mathrm{mm}$ ) with appropriate mixtures of petroleum ether (PE, boiling range $60-70{ }^{\circ} \mathrm{C}$ ) and ethyl acetate as eluents. Melting points (m.p.) were determined in capillary tubes with a Büchi B-520 instrument and 
were not corrected. FTIR spectra were recorded with a Bruker "Alpha-T" spectro-meter with solid compounds measured as $\mathrm{KBr}$ pellets. ATR-IR spectra were measured on the same instrument with a Bruker "Alpha Platinum ATR" single reflection diamond ATR module. ${ }^{1} \mathrm{H}$ NMR and ${ }^{13} \mathrm{C}$ NMR spectra at 600 and $150 \mathrm{MHz}$, respectively, were recorded with an "Avance III" $600 \mathrm{MHz}$ FT-NMR spectrometer (Bruker, Rheinstetten, Germany). ${ }^{1} \mathrm{H}$ NMR and ${ }^{13} \mathrm{C}$ NMR spectra at 400 and $100 \mathrm{MHz}$, respectively, were recorded with an "Avance" $400 \mathrm{MHz}$ FT-NMR spectrometer (also Bruker). ${ }^{1} \mathrm{H}$ NMR and ${ }^{13} \mathrm{C}$ NMR spectra at 200 and $50 \mathrm{MHz}$, respectively, were recorded with an DPX 200 spectrometer (also Bruker). ${ }^{14} \mathrm{~N}$ and ${ }^{15} \mathrm{~N}$ NMR spectra were measured at their appropriate resonance frequency on the aforementioned spectrometers; ${ }^{15} \mathrm{~N}$ measurements were taken as gs- ${ }^{1} \mathrm{H}_{1}{ }^{15} \mathrm{~N}-\mathrm{HSQC}$ or $-\mathrm{HMBC}$ experiments with inverse detection. ${ }^{1} \mathrm{H}$ and ${ }^{13} \mathrm{C}$ NMR spectra were referenced to the residual solvent peak: $\mathrm{CDCl}_{3}, \delta=7.26\left({ }^{1} \mathrm{H}\right)$ and $77.0 \mathrm{ppm}\left({ }^{13} \mathrm{C}\right)$; DMSO- $\mathrm{d}_{6}, \delta=2.50\left({ }^{1} \mathrm{H}\right)$, and $39.7 \mathrm{ppm}\left({ }^{13} \mathrm{C}\right)$. Mass spectra were obtained with a Hewlett-Packard MS 5989B spectrometer, usually in direct mode with electron impact $(70 \mathrm{eV})$. For chlorinated and brominated compounds, all peak values of molecular ions and fragments refer to the isotope ${ }^{35} \mathrm{Cl}$ and ${ }^{79} \mathrm{Br}$. High resolution mass spectra were recorded with a Waters mass spectrometer "VG Autospec" (EI), with a WATERS mass spectrometer "Q-Tof Premier" coupled with a Waters "Acquity UPLC" (ESI), or with a Micromass mass spectrometer "LCT" coupled with a Waters "Alliance 2965 HPLC" (ESI) at the Institute of Organic Chemistry, Leibniz University of Hannover and at the Georg-August University of Göttingen.

\subsection{Synthesis}

Pentachloro-2-nitro-1,3-butadiene (1) was prepared from 2H-pentachloro-1,3-butadiene in $53 \%$ yield (b.p. $69-71{ }^{\circ} \mathrm{C} / 1$ mbar) according to the literature $[3,4] . \quad(Z)$ and (E)-4-Bromotetrachloro-2-nitrobuta-1,3-diene (2) was obtained from (Z)- and (E)-1-bromotetrachlorobuta-1,3-diene in $56 \%$ yield (b.p. $84-86{ }^{\circ} \mathrm{C} / 1.3$ mbar) [5]. (Z)-1,1,4-Trichloro-2,4-dinitrobuta-1,3-diene (3) was synthetized from the (Z)- and (E)-1-bromo-1,4,4-trichlorobuta-1,3-diene in 18\% yield, m.p. 70-71 ${ }^{\circ} \mathrm{C}[6]$.

Synthesis of 4-methyl-2-(2,3,3-trichloro-1-nitroprop-2-en-1-ylidene)-2,3-dihydro-1,3-benzoxazole (4a) (General method). At $-40^{\circ} \mathrm{C}$, a solution of nitrodiene $1(2.71 \mathrm{~g}, 10.0 \mathrm{mmol})$ in $5 \mathrm{~mL}$ methanol $(\mathrm{MeOH})$ was added dropwise to a suspension of 2-amino-3-methylphenol (3.94 g, $32.0 \mathrm{mmol})$ in $30 \mathrm{~mL} \mathrm{MeOH}$ within 5 min. The resulting mixture was kept for $1 \mathrm{~h}$ at this temperature, and was then allowed to reach room temperature (r.t.). After $5 \mathrm{~h}$ stirring, the mixture was poured into a cold solution $\left(0{ }^{\circ} \mathrm{C}\right)$ of $5 \mathrm{~mL}$ conc. $\mathrm{HCl}$ in $250 \mathrm{~mL}$ of water. After $20 \mathrm{~min}$, the precipitate was filtered off, washed with cold water $(3 \times 40$ $\mathrm{mL})$ and diethyl ether $(2 \times 10 \mathrm{~mL})$. Drying in vacuo gave $2.57 \mathrm{~g}$ of oxazole $4 \mathrm{a}$, yield $80 \%$, yellowish solid m.p. $149-151{ }^{\circ} \mathrm{C}$; IR (KBr) $v_{\max }=3089,1612,1382,1079,968,520 \mathrm{~cm}^{-1} ;{ }^{1} \mathrm{H}$ NMR $(200 \mathrm{MHz}$, $\left.\mathrm{CDCl}_{3}\right), \delta=2.61\left(3 \mathrm{H}, \mathrm{s}, \mathrm{CH}_{3} \mathrm{Ph}\right), 7.21-7.33(2 \mathrm{H}, \mathrm{m}, \mathrm{H} \mathrm{Ph}), 7.36-7.41(1 \mathrm{H}, \mathrm{m}, \mathrm{H} \mathrm{Ph}), 11.78(1 \mathrm{H}, \mathrm{s}, \mathrm{NH})$ ppm; ${ }^{13} \mathrm{C} \mathrm{NMR}\left(50 \mathrm{MHz}, \mathrm{CDCl}_{3}\right) \delta=16.6\left(\mathrm{CH}_{3}\right), 107.0\left(\mathrm{C} \mathrm{NO}_{2}\right), 108.7(\mathrm{CH}), 120.2\left(\mathrm{CCl}_{2}\right), 123.4(\mathrm{C} \mathrm{Me})$, $125.4(\mathrm{CH}), 127.4,127.6(\mathrm{CH}), 128.2,146.6,159.0 \mathrm{ppm} ; \mathrm{MS} \mathrm{m} / z\left(I_{\text {rel }}, \%\right): 320\left[\mathrm{M}^{+}\right](4), 285\left[\mathrm{M}-\mathrm{Cl}^{+}\right.$ (10), $274\left[\mathrm{M}-N O_{2}\right]^{+}$(65), $239\left[\mathrm{M}-\mathrm{Cl}-\mathrm{NO}_{2}\right]^{+}(100) ; \mathrm{HRMS}\left(\mathrm{ESI}^{-}\right) \mathrm{m} / \mathrm{z}$ calcd for $\mathrm{C}_{11} \mathrm{H}_{6} \mathrm{~N}_{2} \mathrm{O}_{3} \mathrm{Cl}_{3}[\mathrm{M}-\mathrm{H}]^{-}$: 318.9444; found: 318.9446 .

5-Methyl-2-(2,3,3-trichloro-1-nitroprop-2-en-1-ylidene)-2,3-dihydro-1,3-benzoxazole (4b). Same procedure as for 4a, but using 2-amino-4-methylphenol (3.94 g, $32.0 \mathrm{mmol})$. Yield $2.18 \mathrm{~g}(68 \%)$, yellowish solid, m.p. 168-170 ${ }^{\circ} \mathrm{C}$. IR $(\mathrm{KBr}) v_{\max }=3380,1612,1434,1375,1056,922 \mathrm{~cm}^{-1} .{ }^{1} \mathrm{H}$ NMR $\left(400 \mathrm{MHz}, \mathrm{DMSO}-\mathrm{d}_{6}\right)$ $\delta=2.42\left(3 \mathrm{H}, \mathrm{s}, \mathrm{CH}_{3} \mathrm{Ph}\right), 7.20(1 \mathrm{H}, \mathrm{d}, J=8.3 \mathrm{~Hz}, \mathrm{H} \mathrm{Ph}), 7.36(1 \mathrm{H}, \mathrm{s}, \mathrm{H} \mathrm{Ph}), 7.69(1 \mathrm{H}, \mathrm{d}, J=8.3 \mathrm{~Hz}, \mathrm{H}$ $\mathrm{Ph}), 13.64(1 \mathrm{H}, \mathrm{s}, \mathrm{NH}) \mathrm{ppm} ;{ }^{13} \mathrm{C}$ NMR $\left(100 \mathrm{MHz}, \mathrm{DMSO}-\mathrm{d}_{6}\right) \delta=21.2\left(\mathrm{CH}_{3}\right), 105.4\left(\mathrm{C} \mathrm{NO}_{2}\right), 111.0(\mathrm{CH})$, $113.6(\mathrm{CH}), 122.1\left(\mathrm{CCl}_{2}\right), 126.0(\mathrm{CH}), 126.6,129.8,136.4,144.8,158.0(\mathrm{NCO}) \mathrm{ppm}$. MS m/z $\left(I_{\text {rel }}, \%\right): 320$ $\left[\mathrm{M}^{+}\right]$(2), $285[\mathrm{M}-\mathrm{Cl}]^{+}(4), 274\left[\mathrm{M}-\mathrm{NO}_{2}\right]^{+}(55), 239\left[\mathrm{M}-\mathrm{Cl}_{-}-\mathrm{NO}_{2}\right]^{+}(100), 204\left[\mathrm{M}-2 \mathrm{Cl}-\mathrm{NO}_{2}\right]^{+}(14) ; \mathrm{HRMS}$ $\left(\mathrm{ESI}^{-}\right) \mathrm{m} / \mathrm{z}$ calcd for $\mathrm{C}_{11} \mathrm{H}_{6} \mathrm{~N}_{2} \mathrm{O}_{3} \mathrm{Cl}_{3}[\mathrm{M}-\mathrm{H}]^{-}:$318.9453; found: 318.9444 .

Synthesis of [\{1-(1,3-benzoxazol-2-yl)-3,3-dichloro-2-[4-(dimethylamino)pyridinium-1-yl]prop-2-en-1-ylidene\}(oxido)-l5-azanylloxidanide (5a) (General method). To a suspension of benzoxazole $4 \mathbf{b}$ ( $0.322 \mathrm{~g}$, 
$1.00 \mathrm{mmol}$ ) in $15 \mathrm{~mL} \mathrm{MeOH}$ at $0{ }^{\circ} \mathrm{C}$, a solution of 4-dimethylaminopyridine (DMAP) (0.257 $\mathrm{g}, 2.1$ $\mathrm{mmol}$ ) in $3 \mathrm{~mL} \mathrm{MeOH}$ was added dropwise. The mixture was stirred for $1 \mathrm{~h}$ at $0{ }^{\circ} \mathrm{C}$ and at r.t. for 12 h. Subsequently, the precipitate was filtered off at $0^{\circ} \mathrm{C}$ and washed with water $(2 \times 10 \mathrm{~mL})$ and cold $\mathrm{MeOH}(5 \mathrm{~mL})$. Finally, the product 5a was dried in vacuo. Yield $0.301 \mathrm{~g}(74 \%)$, yellowish solid, m.p. 189-190 ${ }^{\circ} \mathrm{C}$. IR (ATR) $v_{\max }=1641,1531,1355,1139,1055,790 \mathrm{~cm}^{-1} ;{ }^{1} \mathrm{H}$ NMR $\left(400 \mathrm{MHz}, \mathrm{DMSO}-\mathrm{d}_{6}\right) \delta=$ $2.38\left(3 \mathrm{H}, \mathrm{s}, \mathrm{CH}_{3} \mathrm{Ph}\right), 3.23\left(6 \mathrm{H}, \mathrm{s}, \mathrm{N}\left(\mathrm{CH}_{3}\right)_{2}\right), 6.99(1 \mathrm{H}, \mathrm{d}, J=7.8 \mathrm{~Hz}, \mathrm{H} \mathrm{Ph}), 7.06(2 \mathrm{H}, \mathrm{d}, J=6.7 \mathrm{~Hz}, \mathrm{H}$ pyr), $7.33(1 \mathrm{H}, \mathrm{s}, \mathrm{H} \mathrm{Ph}), 7.43(1 \mathrm{H}, \mathrm{d}, J=7.9 \mathrm{~Hz}, \mathrm{H} \mathrm{Ph}), 8.29(2 \mathrm{H}, \mathrm{d}, J=6.7 \mathrm{~Hz}, \mathrm{H}$ pyr $)$ ppm; ${ }^{13} \mathrm{C} \mathrm{NMR}$ $\left(100 \mathrm{MHz}, \mathrm{DMSO}-\mathrm{d}_{6}\right) \delta=39.7\left(\mathrm{CH}_{3}\right), 40.2\left(\mathrm{NCH}_{3}\right), 103.7,107.3(\mathrm{CH}), 109.3(\mathrm{CH}), 117.8(\mathrm{CH}), 122.7$, $123.6(\mathrm{CH}), 133.2,135.8,142.3(\mathrm{CH}), 142.5,147.8,156.4,160.9 \mathrm{ppm} ; \mathrm{MS} \mathrm{m} / \mathrm{z}\left(\mathrm{I}_{\mathrm{rel}}, \%\right): 406\left[\mathrm{M}^{+}\right](1), 273$ [M-benzoxazole-H] $^{+}$(5), 238 [M-benzoxazole-HCl] $^{+}$(3), 122 [DMAP] $^{+}$(73), 100 (100); HRMS $\left(\mathrm{ESI}^{+}\right)$ $m / z$ calcd for $\mathrm{C}_{18} \mathrm{H}_{17} \mathrm{~N}_{4} \mathrm{O}_{3} \mathrm{Cl}_{2}[\mathrm{M}+\mathrm{H}]^{+}$: 407.0672; found: 407.0675 .

[\{1-(1,3-Benzoxazol-2-yl)-3,3-dichloro-2-[4-(morpholin-4-yl)pyridinium-1-yl]prop-2-en-1-ylidene\} (oxido)-15-azanylloxidanide (5b). Following the typical procedure for 5a, using $4 \mathbf{b}(0.322 \mathrm{~g}$, $1.00 \mathrm{mmol})$ and 4-(4-morpholinyl)pyridine $(0.345 \mathrm{~g}, 2.1 \mathrm{mmol})$ at $-18{ }^{\circ} \mathrm{C}$ and holding at this temperature for $3 \mathrm{~h}$. Yield $0.270 \mathrm{~g}(62 \%)$, yellowish solid, m.p. 180-181 ${ }^{\circ} \mathrm{C}$. IR (ATR) $v_{\max }=1640,1548$, 1348, 1253, 1156, $792 \mathrm{~cm}^{-1} .{ }^{1} \mathrm{H}$ NMR $\left(400 \mathrm{MHz}, \mathrm{DMSO}-\mathrm{d}_{6}\right) \delta=2.38\left(3 \mathrm{H}, \mathrm{s}, \mathrm{CH}_{3} \mathrm{Ph}\right), 3.73(8 \mathrm{H}, \mathrm{s}, \mathrm{H}$ morph), $7.00(1 \mathrm{H}, \mathrm{dd}, J=8.1 \mathrm{~Hz}, J=1.3 \mathrm{~Hz}, \mathrm{H} \mathrm{Ph}), 7.25(2 \mathrm{H}, \mathrm{d}, J=7.8, \mathrm{H}$ pyr), $7.34(1 \mathrm{H}, \mathrm{s}, \mathrm{H} \mathrm{Ph}), 7.43$ $(1 \mathrm{H}, \mathrm{d}, J=8.2, \mathrm{H} \mathrm{Ph}), 8.34(2 \mathrm{H}, \mathrm{d}, J=7.6, \mathrm{H}$ pyr $) \mathrm{ppm} ;{ }^{13} \mathrm{C} \mathrm{NMR}\left(100 \mathrm{MHz}, \mathrm{DMSO}-\mathrm{d}_{6}\right) \delta=21.3\left(\mathrm{CH}_{3}\right)$, $46.6\left(\mathrm{~N}\left(\mathrm{CH}_{2}\right)_{2}\right), 65.6\left(\mathrm{O}\left(\mathrm{CH}_{2}\right)_{2}\right), 103.8,107.5(\mathrm{CH}), 109.3(\mathrm{CH}), 117.8(\mathrm{CH}), 122.9,123.9(\mathrm{CH}), 133.2,135.7$, 142.5, $143.0(\mathrm{CH}), 147.8,156.1,160.8$ ppm; MS m/z (I $\left.I_{\text {rel }} \%\right): 448\left[\mathrm{M}^{+}\right]$(1), 325 [M-HCl-morpholine $]^{+}(1)$,

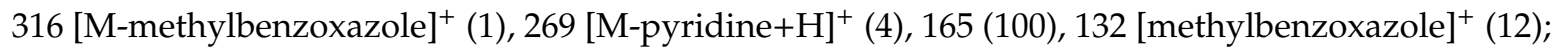
HRMS $\left(\mathrm{ESI}^{+}\right) \mathrm{m} / \mathrm{z}$ calcd for $\mathrm{C}_{20} \mathrm{H}_{19} \mathrm{~N}_{4} \mathrm{O}_{4} \mathrm{Cl}_{2}[\mathrm{M}+\mathrm{H}]^{+}:$449.0778; found: 449.0780 .

1-(Pyridin-4-yl)pyrrolidin-2-one as starting material for the synthesis of azinate $5 \mathrm{c}$ was obtained from 4-aminopyridine and 4-chlorobutanoyl chloride according to the literature [88]. Yield 65\%, colorless liquid. ${ }^{1} \mathrm{H} \mathrm{NMR}\left(600 \mathrm{MHz}, \mathrm{CDCl}_{3}\right) \delta=2.17\left(2 \mathrm{H}, \mathrm{t}, J=7.7 \mathrm{~Hz}, \mathrm{CH}_{2}\right.$ pyrro), $2.61\left(2 \mathrm{H}, \mathrm{t}, J=8.2 \mathrm{~Hz}, \mathrm{CH}_{2}\right.$ pyrro), $3.82\left(2 \mathrm{H}, \mathrm{t}, J=7.1 \mathrm{~Hz}, \mathrm{CH}_{2}\right.$ pyrro), $7.57(2 \mathrm{H}, \mathrm{d}, J=4.9 \mathrm{~Hz}, \mathrm{H}$ pyr $), 8.50(2 \mathrm{H}, \mathrm{d}, J=4.9 \mathrm{~Hz}, \mathrm{H}$ pyr) ppm; ${ }^{13} \mathrm{C}$ NMR $\left(150 \mathrm{MHz}, \mathrm{CDCl}_{3}\right) \delta=17.6\left(\mathrm{CH}_{2}\right), 32.8\left(\mathrm{CH}_{2}\right), 47.4\left(\mathrm{CH}_{2}\right), 112.8(\mathrm{CH}), 145.8,150.4$ $(\mathrm{CH}), 175.2(\mathrm{CO}) \mathrm{ppm} ; \mathrm{MS} m / z\left(\mathrm{I}_{\mathrm{rel}}, \%\right): 162\left[\mathrm{M}^{+}\right](31), 147[\mathrm{M}-\mathrm{OH}]^{+}(25), 119\left[\mathrm{M}-\mathrm{CH}_{2} \mathrm{CHO}\right]^{+}(10), 107$ $\left[\mathrm{M}-\mathrm{CH}_{2} \mathrm{CH}_{2} \mathrm{CO}+\mathrm{H}\right]^{+}(100)$.

[\{1-(1,3-Benzoxazol-2-yl)-3,3-dichloro-2-[4-(2-oxopyrrolidin-1-yl)pyridinium-1-yl]prop-2-en-1-ylidene\}(oxido)-15-azanyl]oxidanide (5c). Same procedure as for 5a, using 1-(pyridin-4-yl)pyrrolidin-2-one (0.341 $\mathrm{g}, 2.1 \mathrm{mmol})$ at $-18{ }^{\circ} \mathrm{C}$ and holding this temperature for $2 \mathrm{~h}$. Stirring was continued for $24 \mathrm{~h}$ at r.t. Yield 67\%, yellow solid, m.p. $157-158^{\circ} \mathrm{C}$. IR (ATR) $v_{\max }=1733,1627,1550,1349,1257,1157 \mathrm{~cm}^{-1} .{ }^{1} \mathrm{H}$ NMR $\left(600 \mathrm{MHz}, \mathrm{DMSO}_{6}\right) \delta=2.13\left(2 \mathrm{H}, \mathrm{t}, J=7.6 \mathrm{~Hz}, \mathrm{CH}_{2}\right.$ pyrro), $2.39\left(3 \mathrm{H}, \mathrm{s}, \mathrm{CH}_{3} \mathrm{Ph}\right), 2.67(2 \mathrm{H}, \mathrm{t}, J=$ $8.0 \mathrm{~Hz}, \mathrm{CH}_{2}$ pyrro), $3.99\left(2 \mathrm{H}, \mathrm{t}, J=7.2 \mathrm{~Hz}, \mathrm{CH}_{2}\right.$ pyrro), $7.01(1 \mathrm{H}, \mathrm{dd}, J=8.2 \mathrm{~Hz}, J=0.8 \mathrm{~Hz}, \mathrm{H} \mathrm{Ph}), 7.35$ (1H, s, H Ph), 7.45 (1H, d, J = 8.2 Hz, H Ph), 8.25 (2H, d, J = 7.0, H pyr), 8.99 (2H, d, J = 7.5 Hz, H pyr) ppm; ${ }^{13} \mathrm{C}$ NMR $\left(150 \mathrm{MHz}, \mathrm{DMSO}-\mathrm{d}_{6}\right) \delta=17.1\left(\mathrm{CH}_{2}\right), 21.2\left(\mathrm{CH}_{3}\right), 32.7\left(\mathrm{CH}_{2}\right), 48.1\left(\mathrm{CH}_{2}\right), 104.0,109.4$ (CH), $113.9(\mathrm{CH}), 117.9(\mathrm{CH}), 123.8(\mathrm{CH}), 124.0,133.3,136.2,142.5,145.7(\mathrm{CH}), 147.8,152.6,160.5,177.5$ ppm; ${ }^{15} \mathrm{~N}$ NMR (43.4 MHz, DMSO-d ${ }_{6}$, doped with nitromethane $\left.(0.0 \mathrm{ppm})\right) \delta=-187.0$ (N-pyridine), -235.2 (N-pyrrolidine) ppm, other N-atoms could not be detected; MS m/z ( $\left.I_{\text {rel }}, \%\right): 446\left[\mathrm{M}^{+}\right](1), 411$

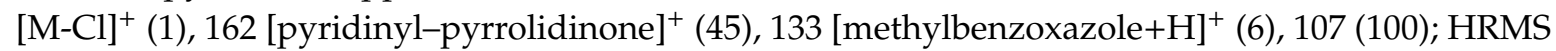
$\left(\mathrm{ESI}^{+}\right) \mathrm{m} / z$ calcd for $\mathrm{C}_{20} \mathrm{H}_{17} \mathrm{~N}_{4} \mathrm{O}_{4} \mathrm{Cl}_{2}[\mathrm{M}+\mathrm{H}]^{+}$: 447.0621 ; found: 447.0623 .

[\{1-(1,3-Benzoxazol-2-yl)-3,3-dichloro-2-[3-(1-methylpyrrolidin-2-yl)pyridinium-1-yl]prop-2-en-1-ylidene\}(oxido)- $\lambda^{5}$-azanyl]oxidanide (5d). To a suspension of benzoxazole $4 \mathbf{b}(0.322 \mathrm{~g}, 1.0 \mathrm{mmol})$ in $\mathrm{MeOH}(10$ $\mathrm{mL})$ at $-18{ }^{\circ} \mathrm{C}$, a solution of (-)-nicotine $(0.324 \mathrm{~g}, 2.0 \mathrm{mmol})$ in $\mathrm{MeOH}(5 \mathrm{~mL})$ was added dropwise. Subsequently, the mixture was stirred at $-18{ }^{\circ} \mathrm{C}$ for $2 \mathrm{~h}$ and at r.t. for $18 \mathrm{~h}$. After completion of the reaction water $(10 \mathrm{~mL}), \mathrm{NaHCO}_{3}(0.184 \mathrm{~g}, 2.2 \mathrm{mmol})$ were added and the mixture was stirred for 10 min, following extraction with chloroform $(3 \times 15 \mathrm{~mL})$. The combined organic phases were dried over 
calcium chloride and purified by column chromatography using DCM-petroleum ether (1:1). The product $5 \mathrm{~d}$ was dried in vacuo. Yield $0.237 \mathrm{~g}(53 \%)$, yellow solid, m.p. $96-98{ }^{\circ} \mathrm{C}$. IR (ATR) $v_{\max }=$ $1622,1516,1354,1259,1141,1062 \mathrm{~cm}^{-1} .{ }^{1} \mathrm{H} \mathrm{NMR}\left(400 \mathrm{MHz}, \mathrm{CDCl}_{3}\right) \delta=1.64-1.77\left(1 \mathrm{H}, \mathrm{m}, \mathrm{CH}_{2}\right.$ pyrro), 1.83-2.01 (2H, m, NCH $), 2.26\left(3 \mathrm{H}, \mathrm{s}, \mathrm{NCH}_{3}\right.$ pyrro), $2.43\left(3 \mathrm{H}, \mathrm{s}, \mathrm{Ph}_{-} \mathrm{CH}_{3}\right), 2.43-2.49\left(1 \mathrm{H}, \mathrm{m}, \mathrm{CH}_{2}\right.$ pyrro), 3.24-3.34 (1H, m, $\mathrm{CH}_{2}$ pyrro), 3.42-3.57 $\left(1 \mathrm{H}, \mathrm{m}, \mathrm{CH}_{2}\right.$ pyrro), 3.63-3.89 $\left(1 \mathrm{H}, \mathrm{m}, \mathrm{CH}_{2}\right.$ pyrro $), 7.00(1 \mathrm{H}$, $\left.\mathrm{dd}, J=1.0 \mathrm{~Hz}, J=8.2 \mathrm{~Hz}, \mathrm{CH}_{3} \mathrm{C}-\mathrm{C} \underline{\mathrm{H}}\right), 7.37(1 \mathrm{H}, \mathrm{s}, \mathrm{H} \mathrm{Ph}), 7.39(1 \mathrm{H}, \mathrm{d}, J=8.2 \mathrm{~Hz}, \mathrm{OCCH}), 7.96(1 \mathrm{H}, \mathrm{dd}, J$ $=5.6 \mathrm{~Hz}, J=7.8 \mathrm{~Hz}, \mathrm{H} \mathrm{pyr}), 8.48(1 \mathrm{H}, \mathrm{d}, J=7.8 \mathrm{~Hz}, \mathrm{H}$ pyr $), 9.03(1 \mathrm{H}, \mathrm{d}, J=5.6 \mathrm{~Hz}, \mathrm{H} \mathrm{pyr}), 9.15(1 \mathrm{H}, \mathrm{s}$, $\mathrm{H}$ pyr) ppm; ${ }^{13} \mathrm{C}$ NMR $\left(100 \mathrm{MHz}, \mathrm{CDCl}_{3}\right) \delta=21.5\left(\mathrm{CH}_{3} \mathrm{Ph}\right), 23.1\left(\mathrm{CH}_{2}\right), 35.8\left(\mathrm{CH}_{2}\right), 40.3\left(\mathrm{CH}_{3}\right.$ pyrro $)$, 56.6 $\left(\mathrm{CH}_{2}\right), 67.1$ (CH pyrro), 104.6 $\left(\mathrm{CNO}_{2}\right), 109.8(\mathrm{CH} \mathrm{Ph}), 118.0(\mathrm{CH} \mathrm{Ph}), 124.0(\mathrm{CH} \mathrm{Ph}), 127.0(\mathrm{CH}$ pyr$)$, $128.5\left(\mathrm{CCl}_{2}\right), 133.7\left(\mathrm{CCH}_{3}\right), 136.3,142.2,143.6(\mathrm{CH}$ pyr $), 145.2(\mathrm{CH}$ pyr $), 145.5(\mathrm{CH}$ pyr $), 145.7,148.3$ (C-pyrro), 160.1 (NCO) ppm; MS (ESI ${ }^{+}$) $\mathrm{m} / z$ calcd for $\mathrm{C}_{21} \mathrm{H}_{21} \mathrm{~N}_{4} \mathrm{O}_{3} \mathrm{Cl}_{2}[\mathrm{M}+\mathrm{H}]^{+}$: 447.1; found 447.1.

2-[3-Bromo-2,3-dichloro-1-nitroprop-2-en-1-ylidene]-4-methyl-2,3-dihydro-1,3-benzoxazole (6). Same proce-dure as for $4 \mathrm{a}$, but starting from $2(316 \mathrm{mg}, 1.00 \mathrm{mmol}$, a $47: 53$ mixture of isomers) using 2-amino-3-methylphenol (394 mg, $3.20 \mathrm{mmol})$. A 1:1 mixture of isomers was obtained. Yield 73\%, yellowish solid, m.p. $145-146{ }^{\circ} \mathrm{C}$. IR $(\mathrm{KBr}) v_{\max }=3329,1620,1571\left(\mathrm{NO}_{2}\right), 1376,1320,1047 \mathrm{~cm}^{-1} ;{ }^{1} \mathrm{H}$ NMR (200 MHz, $\left.\mathrm{CDCl}_{3}\right) \delta=2.61\left(3 \mathrm{H}, \mathrm{s}, \mathrm{CH}_{3} \mathrm{Ph}\right), 7.22-7.41(3 \mathrm{H}, \mathrm{m}, \mathrm{H} \mathrm{Ph}), 11.77(1 \mathrm{H}, \mathrm{s}, \mathrm{NH}) \mathrm{ppm}$; ${ }^{13} \mathrm{C} \mathrm{NMR}\left(50 \mathrm{MHz}, \mathrm{CDCl}_{3}\right) \delta=16.6\left(\mathrm{CH}_{3}\right) ; 106.9\left(\mathrm{CNO}_{2}\right) ; 108.7(\mathrm{CH}) ; 114.9,115.8(\mathrm{CClBr}) ; 121.8,123.7$ (CCl); $123.4(\mathrm{C} \mathrm{Me}) ; 125.4(\mathrm{CH}), 127.4,127.6(\mathrm{CH}), 146.7,158.7,158.9(\mathrm{NCO}) \mathrm{ppm}$; MS m/z (I rel $\left._{1}, \%\right)$ : $364\left[\mathrm{M}^{+}\right](2), 329[\mathrm{M}-\mathrm{Cl}]^{+}(4), 318\left[\mathrm{M}-\mathrm{NO}_{2}\right]^{+}(19), 285[\mathrm{M}-\mathrm{Br}]^{+}(25), 283\left[\mathrm{M}-\mathrm{Cl}_{-}-\mathrm{NO}_{2}\right]^{+}(50), 239$ [M-Br-NO$]^{+}$(100); HRMS (ESI ${ }^{-}$) $\mathrm{m} / \mathrm{z}$ calcd for $\mathrm{C}_{11} \mathrm{H}_{6} \mathrm{~N}_{2} \mathrm{O}_{3} \mathrm{Cl}_{2} \mathrm{Br}[\mathrm{M}-\mathrm{H}]^{-}: 362.8944$; found: 362.8948 .

2-((Z)-3-chloro-1,3-dinitroallylidene)-5-methyl-2,3-dihydro-1H-benzo[d]imidazole (7a). Same procedure as for $4 a$, but starting from $3(247 \mathrm{mg}$, $1.00 \mathrm{mmol}$, only Z-isomer) using 4-methylbenzene-1,2-diamine (257 $\mathrm{mg}, 2.10 \mathrm{mmol})$. Yield $93 \%$, red solid, m.p. $163-165^{\circ} \mathrm{C}$. IR $(\mathrm{KBr}) v_{\max }=3330,1602,1577\left(\mathrm{NO}_{2}\right), 1410$, $1268,987 \mathrm{~cm}^{-1} .{ }^{1} \mathrm{H}$ NMR spectrum $\left(200 \mathrm{MHz}, \mathrm{DMSO}_{6}\right) \delta=2.49\left(3 \mathrm{H}, \mathrm{s}, \mathrm{CH}_{3} \mathrm{Ph}\right), 7.36(1 \mathrm{H}, \mathrm{d}, J=8.5$ $\mathrm{Hz}, \mathrm{H} \mathrm{Ph}), 7.58(1 \mathrm{H}, \mathrm{s}, \mathrm{H} \mathrm{Ph}), 7.67(1 \mathrm{H}, \mathrm{d}, J=8.5 \mathrm{~Hz}, \mathrm{H} \mathrm{Ph}), 9.14(1 \mathrm{H}, \mathrm{s}, \mathrm{CH}), 14.45(2 \mathrm{H}, \mathrm{s}, \mathrm{NH}) \mathrm{ppm}$; ${ }^{13} \mathrm{C}$ NMR $\left(50 \mathrm{MHz}, \mathrm{DMSO}-\mathrm{d}_{6}\right) \delta=21.4\left(\mathrm{CH}_{3}\right), 106.9\left(\mathrm{C} \mathrm{NO}_{2}\right), 113.4(\mathrm{CH}), 113.6(\mathrm{CH}), 120.1\left(\mathrm{CClNO}_{2}\right)$, $127.5(\mathrm{CH}), 129.1$ (C Me), $129.2(\mathrm{CH}), 131.2,136.1,142.3(\mathrm{NCN}) \mathrm{ppm} ; \mathrm{MS}$ m/z (I rel $\left._{1} \%\right): 296\left[\mathrm{M}^{+}\right]$(5), 250 $\left[\mathrm{M}-N O_{2}\right]^{+}(55), 233\left[\mathrm{M}-\mathrm{NO}_{2}-\mathrm{OH}\right]^{+}(12), 204\left[\mathrm{M}-2 \mathrm{NO}_{2}\right]^{+}(60), 169\left[\mathrm{M}-2 \mathrm{NO}_{2}-\mathrm{Cl}\right]^{+}$(48), 157 (100); HRMS (ESI ${ }^{-}$) $\mathrm{m} / z$ calcd for $\mathrm{C}_{11} \mathrm{H}_{8} \mathrm{~N}_{4} \mathrm{O}_{4} \mathrm{Cl}[\mathrm{M}-\mathrm{H}]^{-}:$295.0240; found: 295.0248 .

2-(3-Chloro-1,3-dinitroprop-2-en-1-ylidene)-5-methyl-2,3-dihydro-1,3-benzoxazole (7b). Same procedure as for 4a, but using 3 (247 mg, $1.00 \mathrm{mmol}$, only Z-isomer) and 2-amino-4-methylphenol (394 mg, 3.20 mmol). Yield $67 \%$, yellow solid, m.p. $110-112{ }^{\circ} \mathrm{C}$. IR $(\mathrm{KBr}) v_{\max }=3250,1602,1545\left(\mathrm{NO}_{2}\right), 1289,1065$, $871 \mathrm{~cm}^{-1} .{ }^{1} \mathrm{H}$ NMR $\left(200 \mathrm{MHz}, \mathrm{DMSO}-\mathrm{d}_{6}\right) \delta=2.45\left(3 \mathrm{H}, \mathrm{s}, \mathrm{CH}_{3} \mathrm{Ph}\right), 7.25(1 \mathrm{H}, \mathrm{d}, J=8.3 \mathrm{~Hz}, \mathrm{H} \mathrm{Ph}), 7.55$ $(1 \mathrm{H}, \mathrm{s}, \mathrm{H} \mathrm{Ph}), 7.63(1 \mathrm{H}, \mathrm{d}, J=8.5 \mathrm{~Hz}, \mathrm{H} \mathrm{Ph}), 9.09(1 \mathrm{H}, \mathrm{s}, \mathrm{CH}), 10.66(1 \mathrm{H}, \mathrm{s}, \mathrm{NH}) ;{ }^{13} \mathrm{C} \mathrm{NMR}(50 \mathrm{MHz}$, DMSO-d $\left.{ }_{6}\right) \delta=21.2\left(\mathrm{CH}_{3}\right), 110.8(\mathrm{CH}), 112.5\left(\mathrm{CNO}_{2}\right), 118.5\left(\mathrm{CClNO}_{2}\right), 119.1(\mathrm{CH}), 126.8(\mathrm{CH}), 129.5$ $(\mathrm{CH}), 134.5(\mathrm{C} \mathrm{Me}), 139.5,148.2,157.2(\mathrm{NCO}) \mathrm{ppm} ; \mathrm{MS} \mathrm{m} / z\left(I_{\text {rel }} \%\right): 297\left[\mathrm{M}^{+}\right](3), 251\left[\mathrm{M}-\mathrm{NO}_{2}\right]^{+}$ (45), $234\left[\mathrm{M}-\mathrm{NO}_{2}-\mathrm{OH}\right]^{+}$(13), 158 (100); $\mathrm{HRMS}\left(\mathrm{ESI}^{-}\right) \mathrm{m} / \mathrm{z}$ calcd for $\mathrm{C}_{11} \mathrm{H}_{7} \mathrm{~N}_{3} \mathrm{O}_{5} \mathrm{Cl}[\mathrm{M}-\mathrm{H}]^{-}:$296.0072; found: 296.0070 .

2-(3-Chloro-1,3-dinitroprop-2-en-1-ylidene)-4-methyl-2,3-dihydro-1,3-benzoxazole (7c). Same procedure as for $4 a$, using 3 ( $247 \mathrm{mg}, 1.00 \mathrm{mmol}$, only Z-isomer) and 2-amino-3-methylphenol (394 mg, $3.20 \mathrm{mmol}$ ). Yield 85\%, yellow solid, m.p. $102-103^{\circ} \mathrm{C}$. IR $(\mathrm{KBr}) v_{\max }=3254,1625,1579\left(\mathrm{NO}_{2}\right), 1520,1077,646$ $\mathrm{cm}^{-1} .{ }^{1} \mathrm{H}$ NMR $\left(200 \mathrm{MHz}, \mathrm{DMSO}-\mathrm{d}_{6}\right) \delta=2.53\left(3 \mathrm{H}, \mathrm{s}, \mathrm{CH}_{3} \mathrm{Ph}\right), 7.19(1 \mathrm{H}, \mathrm{d}, J=7.5 \mathrm{~Hz}, \mathrm{H} \mathrm{Ph}), 7.30$ $(1 \mathrm{H}, \mathrm{t}, J=7.5 \mathrm{~Hz}, \mathrm{H} \mathrm{Ph}), 7.52(1 \mathrm{H}, \mathrm{d}, J=8.0 \mathrm{~Hz}, \mathrm{H} \mathrm{Ph}), 9.20(1 \mathrm{H}, \mathrm{s}, \mathrm{CH}), 10.19(1 \mathrm{H}, \mathrm{s}, \mathrm{NH}) \mathrm{ppm} ;{ }^{13} \mathrm{C}$ NMR (50 MHz, DMSO-d 6$) \delta=16.5\left(\mathrm{CH}_{3}\right), 108.5(\mathrm{CH}), 113.6\left(\mathrm{CNO}_{2}\right), 115.9\left(\mathrm{CClNO}_{2}\right), 125.0(\mathrm{CH}), 125.5$

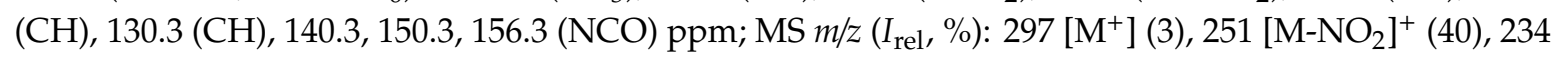
[M-NO$\left.{ }_{2}-\mathrm{OH}\right]^{+}$(12), 158 (100); $\mathrm{MS}\left(\mathrm{ESI}^{-}\right) \mathrm{m} / z$ calcd for $\mathrm{C}_{11} \mathrm{H}_{7} \mathrm{~N}_{3} \mathrm{O}_{5} \mathrm{Cl}[\mathrm{M}-\mathrm{H}]^{-}:$296.0; found: 296.0.

Synthesis of 2-(3-bromo-2,3-dichloro-1-nitroprop-2-en-1-ylidene)-1,3-diphenylimidazolidine (8b) (General method). To a solution of $N, N^{\prime}$-diphenylethane-1,2-diamine $(0.446 \mathrm{~g}, 2.1 \mathrm{mmol})$ in $10 \mathrm{~mL} \mathrm{MeOH}$ at 
$-40{ }^{\circ} \mathrm{C}$ a solution of nitrodiene $2(0.316 \mathrm{~g}, 1.0 \mathrm{mmol})$ in $5 \mathrm{~mL} \mathrm{MeOH}$ was added dropwise. After $1 \mathrm{~h}$ of stirring at $-40{ }^{\circ} \mathrm{C}$, the solution was allowed to reach r.t. and stirred for another $5 \mathrm{~h}$. The precipitate was filtered off, washed with water $(3 \times 20 \mathrm{~mL}), \mathrm{MeOH}(1 \times 10 \mathrm{~mL})$, diethyl ether $(2 \times 10 \mathrm{~mL})$ and dried in vacuo. A 1:1 mixture of isomers was obtained. Yield $0.410 \mathrm{~g}(90 \%)$, yellow solid, m.p. $222-223$ ${ }^{\circ} \mathrm{C}$. IR $(\mathrm{KBr}) v_{\max }=3455,3059,1594,1523,1295,761 \mathrm{~cm}^{-1} .{ }^{1} \mathrm{H}$ NMR $\left(200 \mathrm{MHz}, \mathrm{DMSO}-\mathrm{d}_{6}\right) \delta=4.37$ $\left(4 \mathrm{H}, \mathrm{s}, \mathrm{CH}_{2}\right), 7.25-7.47(10 \mathrm{H}, \mathrm{m}, \mathrm{H} \mathrm{Ph}) \mathrm{ppm} ;{ }^{13} \mathrm{C}$ NMR $\left(50 \mathrm{MHz}, \mathrm{DMSO}-\mathrm{d}_{6}\right) \delta=51.0,50.9\left(\mathrm{CH}_{2}\right), 104.4$, $105.9\left(\mathrm{CNO}_{2}\right), 107.2,107.7(\mathrm{CBrCl}), 122.4,122.5(\mathrm{CH}), 127.0(\mathrm{CH}), 127.8,129.5,129.6(\mathrm{CH}), 139.9,140.0$ (NC Ph), 155.6, 155.7 (NCN) ppm; MS m/z (I rel $\left._{1} \%\right): 453\left[\mathrm{M}^{+}\right]$(3), $418[\mathrm{M}-\mathrm{Cl}]^{+}$(3), $374[\mathrm{M}-\mathrm{Br}]^{+}(45)$, 339 [M-Cl-Br] $^{+}(8), 279$ [M-C $\left.\mathrm{Cl}_{2} \mathrm{Cl}_{2} \mathrm{Br}\right]^{+}(100)$; HRMS $\left(\mathrm{ESI}^{+}\right) \mathrm{m} / \mathrm{z}$ calcd for $\mathrm{C}_{18} \mathrm{H}_{15} \mathrm{~N}_{3} \mathrm{O}_{2} \mathrm{Cl}_{2} \mathrm{Br}[\mathrm{M}+\mathrm{H}]^{+}$: 453.9719; found: 453.9728 .

2-(3-Chloro-1,3-dinitroprop-2-en-1-ylidene)-1,3-diphenylimidazolidine (8c). Same procedure as for $\mathbf{8 b}$, using dinitrodiene $3(0.247 \mathrm{~g}, 1.0 \mathrm{mmol})$. Yield $86 \%$, yellow solid, m.p. $236-237^{\circ} \mathrm{C}$. IR $(\mathrm{KBr}) v_{\max }=3054$, 1570, 1491, 1349, 1193, $999 \mathrm{~cm}^{-1} .{ }^{1} \mathrm{H}$ NMR $\left(200 \mathrm{MHz}, \mathrm{CDCl}_{3}\right) \delta=4.58\left(4 \mathrm{H}, \mathrm{s}, \mathrm{CH}_{2}\right), 7.29-7.33(4 \mathrm{H}, \mathrm{m}, \mathrm{H}$ $\mathrm{Ph}), 7.39-7.45(6 \mathrm{H}, \mathrm{m}, \mathrm{H} \mathrm{Ph}), 8.69(1 \mathrm{H}, \mathrm{s}, \mathrm{CH}) \mathrm{ppm} ;{ }^{13} \mathrm{C}$ NMR $\left(50 \mathrm{MHz}, \mathrm{DMSO}_{6}\right) \delta=50.6\left(\mathrm{CH}_{2}\right), 105.1$, $117.8\left(\mathrm{CClNO}_{2}\right), 123.2(\mathrm{CH}), 128.4(\mathrm{CH}), 129.0(\mathrm{CH}), 130.0(\mathrm{CH}), 136.7(\mathrm{NC} \mathrm{Ph}), 158.7(\mathrm{NCN}) \mathrm{ppm} ; \mathrm{MS}$ $m / z\left(I_{\text {rel }}, \%\right): 386\left[\mathrm{M}^{+}\right](20), 340\left[\mathrm{M}-\mathrm{NO}_{2}\right]^{+}(60), 305\left[\mathrm{M}-\mathrm{NO}_{2}-\mathrm{Cl}\right]^{+}(3), 281\left[\mathrm{M}-\left(\mathrm{CH}=\mathrm{CClNO}_{2}\right)+\mathrm{H}\right]^{+}(20)$, $247\left[\mathrm{M}-(\mathrm{CH}=\mathrm{CClNO})_{2}-2 \mathrm{OH}\right]^{+}(100)$; $\mathrm{HRMS}\left(\mathrm{ESI}^{+}\right) \mathrm{m} / z$ calcd for $\mathrm{C}_{18} \mathrm{H}_{16} \mathrm{~N}_{4} \mathrm{O}_{4} \mathrm{Cl}[\mathrm{M}+\mathrm{H}]^{+}$: 387.0855; found: 387.0865 .

Synthesis of 2-[3,3-dichloro-2-nitro-1-(pyrrolidin-1-yl)prop-2-en-1-ylidene]-1,3-diphenylimidazolidine (9). To a suspension of imidazolidine $8 \mathrm{a}(0.387 \mathrm{~g}, 1.0 \mathrm{mmol})$ in $10 \mathrm{~mL} \mathrm{MeOH}$ at r.t. a solution of pyrrolidine $(0.356 \mathrm{~g}, 5.0 \mathrm{mmol})$ in $5 \mathrm{~mL} \mathrm{MeOH}$ was added. Subsequently, the mixture was stirred for $3 \mathrm{~h}$ at r.t. and $4 \mathrm{~h}$ at reflux. After cooling to $10{ }^{\circ} \mathrm{C}$, the $\mathrm{pH}$ was adjusted to $6-7$ with $\mathrm{HCl}(5 \%)$. The resulting precipitate was filtered off and washed with water $(3 \times 20 \mathrm{~mL}), \mathrm{MeOH}(2 \times 10 \mathrm{~mL})$ and dried in vacuo. Yield $0.223 \mathrm{~g}(50 \%)$, yellow solid, m.p. $156-159^{\circ} \mathrm{C}$. IR $(\mathrm{KBr}) v_{\max }=2968,2868,1597,1497$, 1347, $908 \mathrm{~cm}^{-1} .{ }^{1} \mathrm{H}$ NMR $\left(200 \mathrm{MHz}, \mathrm{DMSO}_{6}\right) \delta=1.52-1.56\left(4 \mathrm{H}, \mathrm{m}, \mathrm{CH}_{2} \mathrm{pyr}\right), 2.68-2.73(4 \mathrm{H}, \mathrm{m}$, $\mathrm{CH}_{2}$ pyr), $4.29\left(4 \mathrm{H}, \mathrm{s}, \mathrm{CH}_{2} \mathrm{im}\right), 7.21-7.44(10 \mathrm{H}, \mathrm{m}, \mathrm{H} \mathrm{Ph}) \mathrm{ppm} ;{ }^{13} \mathrm{C}$ NMR $\left(50 \mathrm{MHz}, \mathrm{DMSO}-\mathrm{d}_{6}\right) \delta=$ $25.0\left(\mathrm{CH}_{2}\right), 50.1\left(\mathrm{CH}_{2}\right), 51.0\left(\mathrm{CH}_{2}\right), 98.6\left(\mathrm{CCl}_{2}\right), 104.7\left(\mathrm{CNO}_{2}\right), 122.8(\mathrm{CH}), 126.7(\mathrm{CH}), 129.2(\mathrm{CH})$,

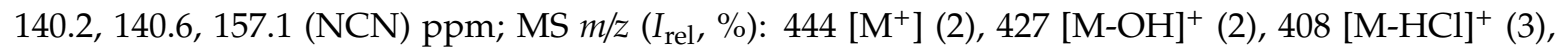

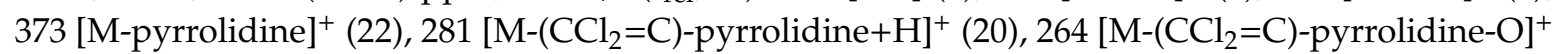
(85), 248 [M-( $\left.\mathrm{CCl}_{2}=\mathrm{C}\right)$-pyrrolidine-2O] ${ }^{+}(100)$; HRMS $\left(\mathrm{ESI}^{+}\right) \mathrm{m} / z$ calcd for $\mathrm{C}_{22} \mathrm{H}_{23} \mathrm{~N}_{4} \mathrm{O}_{2} \mathrm{Cl}_{2}[\mathrm{M}+\mathrm{H}]^{+}$: 445.1193; found: 445.1190 .

Synthesis of ethyl 1-(1,1-dichloro-3-\{1-[(6-chloropyridin-3-yl)methyl]imidazolidin-2-ylidene\}-3 -nitroprop-1-en-2-yl)piperidine-4-carboxylate (12a) (General method). To a suspension of compound 11a $(0.384 \mathrm{~g}, 1.0 \mathrm{mmol})$ and piperidine-4-carboxylic acid ethyl ester $(0.393 \mathrm{~g}, 2.5 \mathrm{mmol})$ in $15 \mathrm{~mL} \mathrm{MeOH}$ at r.t., a solution of sodium ethanolate $(0.177 \mathrm{~g}, 2.6 \mathrm{mmol})$ in $5 \mathrm{~mL} \mathrm{MeOH}$ was added, and stirred for $24 \mathrm{~h}$ at $35^{\circ} \mathrm{C}$. At r.t. 3 drops of conc. $\mathrm{HCl}$ were added before adding $50 \mathrm{~mL}$ cold water. The resulting precipitate was filtered off and washed with cold $\mathrm{MeOH}(2 \times 7 \mathrm{~mL})$ and water $(3 \times 10 \mathrm{~mL})$. The product was dried in vacuo. Yield $0.303 \mathrm{~g}(60 \%)$, yellow solid, m.p. $60-62{ }^{\circ} \mathrm{C}$. IR (KBr) $v_{\max }=$ $3319,1726,1561,1319,1177,1043 \mathrm{~cm}^{-1} .{ }^{1} \mathrm{H}$ NMR $(200 \mathrm{MHz}$, DMSO-d 6$) \delta=1.16\left(3 \mathrm{H}, \mathrm{t}, J=6.9 \mathrm{~Hz}, \mathrm{CH}_{3}\right.$ Et), $1.53-1.76\left(4 \mathrm{H}, \mathrm{m}, \mathrm{CH}_{2}\right.$ pip), 2.28-2.42 (1H, m, $\mathrm{CH}$ pip), 2.73 (4H, s, $\mathrm{CH}_{2}$ pip), 3.35 (2H, s, $\left.\mathrm{CH}_{2} \mathrm{imi}\right)$, $3.67\left(2 \mathrm{H}, \mathrm{s}, \mathrm{CH}_{2} \mathrm{imi}\right), 4.06\left(2 \mathrm{H}, \mathrm{q}, J=6.9 \mathrm{~Hz}, \mathrm{CH}_{2} \mathrm{Et}\right), 4.37-4.47(2 \mathrm{H}, \mathrm{m}$, pyr-CH $-\mathrm{imi}), 7.56(1 \mathrm{H}, \mathrm{d}, J=$ $8.1 \mathrm{~Hz}, \mathrm{H}$ pyr $), 7.81(1 \mathrm{H}, \mathrm{d}, J=8.2 \mathrm{~Hz}, \mathrm{H}$ pyr $), 8.36(1 \mathrm{H}, \mathrm{s}, \mathrm{H}$ pyr $), 9.43(1 \mathrm{H}, \mathrm{s}, \mathrm{NH}) \mathrm{ppm} ;{ }^{13} \mathrm{C} \mathrm{NMR}(50$ MHz, DMSO-d 6$) \delta=14.3\left(\mathrm{CH}_{3}\right), 28.4\left(\mathrm{CH}_{2}\right), 40.5(\mathrm{CH}), 42.2\left(\mathrm{CH}_{2}\right), 48.0\left(\mathrm{CH}_{2}\right.$ pip $), 48.4\left(\mathrm{CH}_{2}\right), 49.8$ $\left(\mathrm{CH}_{2}\right), 60.1\left(\mathrm{CH}_{2}\right), 102.8\left(\mathrm{CNO}_{2}\right), 103.6\left(\mathrm{CCl}_{2}\right), 124.4(\mathrm{CH}), 131.4,138.8(\mathrm{CH}), 141.3,148.9(\mathrm{CH}), 149.7$, 160.1, $174.4 \mathrm{ppm} ; \mathrm{MS} \mathrm{m} / z\left(\mathrm{I}_{\text {rel }}, \%\right): 503\left[\mathrm{M}^{+}\right]$(3), $467[\mathrm{M}-\mathrm{HCl}]^{+}$(3), 377 [M-CH$_{2}$ pyridine $^{+}$(2), 254 [M- $_{2} \mathrm{Cl}_{2}$-piperidine] ${ }^{+}(18), 126\left[\mathrm{CH}_{2}\right.$ pyridine] $\left.{ }^{+}(100) ; \mathrm{HRMS}_{(\mathrm{ESI}}{ }^{-}\right) \mathrm{m} / z$ calcd for $\mathrm{C}_{20} \mathrm{H}_{23} \mathrm{~N}_{5} \mathrm{O}_{4} \mathrm{Cl}_{3}$ [M $-\mathrm{H}^{-}:$: 502.0821; found: 502.0818 . 
2-(1,1-Dichloro-3-\{1-[(6-chloropyridin-3-yl)methyl]imidazolidin-2-ylidene\}-3-nitroprop-1-en-2-yl)-

1,2,3,4-tetrahydroisoquinoline (12b). Same procedure as for 12a, using 11a $(0.384 \mathrm{~g}, 1.00 \mathrm{mmol})$ and 1,2,3,4-tetrahydroisoquinoline $(0.368 \mathrm{~g}, 2.50 \mathrm{mmol})$ and stirring for $4 \mathrm{~h}$ at $50{ }^{\circ} \mathrm{C}$. Yield $85 \%$, orange solid, m.p. $146-148^{\circ} \mathrm{C}$. IR $(\mathrm{KBr}) v_{\max }=3318,2895,1559,1460,1320,749 \mathrm{~cm}^{-1}$. ${ }^{1} \mathrm{H}$ NMR spectrum (200 MHz, $\left.\mathrm{CDCl}_{3}\right) \delta=2.70-3.16$ (2H, m, $\mathrm{CH}_{2}$ pip), 3.30-3.55 (2H, m, $\mathrm{CH}_{2}$ pip), 3.59-3.90 (4H, m, $\mathrm{CH}_{2}$ imi), $4.08\left(1 \mathrm{H}, \mathrm{d}, \mathrm{J}=15.2 \mathrm{~Hz}, \mathrm{NCH}_{2}\right) ; 4.26-4.38\left(2 \mathrm{H}, \mathrm{m}, \mathrm{CH}_{2} \mathrm{pip}\right), 4.67\left(1 \mathrm{H}, \mathrm{d}, \mathrm{J}=15.2 \mathrm{~Hz}, \mathrm{NCH}_{2}\right)$, 6.91-6.97 (1H, m, H Ph), 7.07-7.15 (3H, m, H Ph), $7.32(1 \mathrm{H}, \mathrm{d}, \mathrm{J}=8.2 \mathrm{~Hz}, \mathrm{H}$ pyr), $7.60(1 \mathrm{H}, \mathrm{dd}, \mathrm{J}=8.3 \mathrm{~Hz}$, $\mathrm{J}=2.5 \mathrm{~Hz}, \mathrm{H}$ pyr $), 8.30(1 \mathrm{H}, \mathrm{d}, \mathrm{J}=2.4 \mathrm{~Hz}, \mathrm{H}$ pyr $), 9.56(1 \mathrm{H}, \mathrm{s}, \mathrm{NH}) \mathrm{ppm} .{ }^{13} \mathrm{C} \mathrm{NMR}\left(50 \mathrm{MHz}, \mathrm{CDCl}_{3}\right) \delta$ $=29.7\left(\mathrm{CH}_{2}\right), 41.7\left(\mathrm{CH}_{2}\right), 48.7\left(\mathrm{CH}_{2}\right), 49.6\left(\mathrm{CH}_{2}\right), 49.9\left(\mathrm{CH}_{2}\right), 51.3\left(\mathrm{CH}_{2}\right), 105.1,105.7,124.7(\mathrm{CH}), 125.7$ (CH), $126.7(\mathrm{CH}), 126.3(\mathrm{CH}), 128.8(\mathrm{CH}), 129.6,133.9,134.9,138.2(\mathrm{CH}), 139.7,148.9(\mathrm{CH}), 151.6,160.2$ ppm; MS m/z (I $\left.\mathrm{rel}_{\mathrm{rel}} \%\right): 479\left[\mathrm{M}^{+}\right](2), 443[\mathrm{M}-\mathrm{HCl}]^{+}(3), 407[\mathrm{M}-2(\mathrm{HCl})]^{+}(5), 254\left[\mathrm{M}-\mathrm{C}_{2} \mathrm{Cl}_{2}\right.$-isoquinoline $+\mathrm{H}^{+}(7), 126$ [Cl-pyr-CH$]^{+}(100)$; HRMS $\left(\mathrm{ESI}^{-}\right) \mathrm{m} / \mathrm{z}$ calcd for $\mathrm{C}_{21} \mathrm{H}_{19} \mathrm{~N}_{5} \mathrm{O}_{2} \mathrm{Cl}_{3}[\mathrm{M}-\mathrm{H}]^{-}: 478.0610$; found: 478.0606 .

2-[(1,1-Dichloro-3-\{1-[(6-chloropyridin-3-yl)methyl]imidazolidin-2-ylidene\}-3-nitroprop-1-en-2-yl)sulfanyl]ethanol (12c). Same procedure as for 12a, using 11a $(0.384 \mathrm{~g}, 1.00 \mathrm{mmol})$, 2-mercaptoethanol $(0.086 \mathrm{~g}$, $1.1 \mathrm{mmol})$ and sodium ethanolate $(0.082 \mathrm{~g}, 1.2 \mathrm{mmol})$ in $\mathrm{EtOH}$. The mixture was stirred for $12 \mathrm{~h}$ at 40 ${ }^{\circ} \mathrm{C}$. Yield $0.268 \mathrm{~g}(63 \%)$, orange solid, m.p. $185-187^{\circ} \mathrm{C}$. IR $(\mathrm{KBr}) v_{\max }=3220,2974,1575,1299,1125$, $844 \mathrm{~cm}^{-1} .{ }^{1} \mathrm{H}$ NMR spectrum $\left(200 \mathrm{MHz}, \mathrm{DMSO}_{6}\right) \delta=2.77\left(2 \mathrm{H}, \mathrm{t}, J=6.4 \mathrm{~Hz}, \mathrm{SCH}_{2}\right), 3.53(2 \mathrm{H}, \mathrm{t}, J$ $\left.=6.4 \mathrm{~Hz}, \underline{\mathrm{CH}}_{2} \mathrm{OH}\right), 3.73\left(4 \mathrm{H}, \mathrm{s}, \mathrm{CH}_{2}\right), 4.30\left(2 \mathrm{H}, \mathrm{s}, \mathrm{NCH}_{2}\right), 4.70(1 \mathrm{H}, \mathrm{s}, \mathrm{OH}), 7.53(1 \mathrm{H}, \mathrm{d}, J=8.3 \mathrm{~Hz})$, $7.75(1 \mathrm{H}, \mathrm{dd}, J=8.3 \mathrm{~Hz}, J=2.5 \mathrm{~Hz}), 8.33(1 \mathrm{H}, \mathrm{d}, J=2.3 \mathrm{~Hz}), 9.55(1 \mathrm{H}, \mathrm{s}, \mathrm{NH}) \mathrm{ppm} ;{ }^{13} \mathrm{C} \mathrm{NMR}(50 \mathrm{MHz}$, DMSO-d $\left.{ }_{6}\right) \delta=34.9\left(\mathrm{CH}_{2}\right), 42.4\left(\mathrm{CH}_{2}\right), 48.9\left(\mathrm{CH}_{2}\right), 50.8\left(\mathrm{CH}_{2}\right), 60.1\left(\mathrm{CH}_{2}\right), 102.8\left(\mathrm{CNO}_{2}\right), 115.1\left(\mathrm{CCl}_{2}\right)$, $124.3(\mathrm{CH}), 131.6,131.9,138.1(\mathrm{CH}), 148.0(\mathrm{CH}), 149.5,159.7 \mathrm{ppm} ; \mathrm{MS} \mathrm{m} / \mathrm{z}\left(\mathrm{I}_{\text {rel }}, \%\right): 424\left[\mathrm{M}^{+}\right](1), 254$

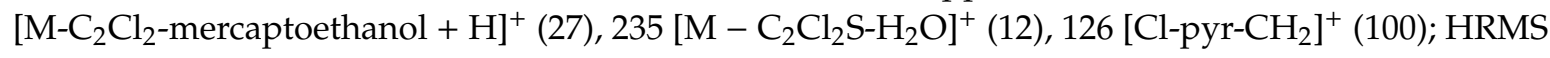
$\left(\mathrm{ESI}^{-}\right) \mathrm{m} / \mathrm{z}$ calcd for $\mathrm{C}_{14} \mathrm{H}_{14} \mathrm{~N}_{4} \mathrm{O}_{3} \mathrm{SCl}_{3}[\mathrm{M}-\mathrm{H}]^{-}:$422.9858; found: 422.9854 .

1,1-Dichloro-3-\{3-[(6-chloropyridin-3-yl)methyl]-1,3-oxazolidin-2-ylidene $\}-N, N$-dimethyl-3-nitroprop-1en-2-amine (12d). Same procedure as for 12a, using pyridine $\mathbf{1 1 b}(0.385 \mathrm{~g}, 1.00 \mathrm{mmol})$ and dimethylamine $(0.366 \mathrm{~g}, 5.00 \mathrm{mmol})$ and stirring at r.t. for $5 \mathrm{~h}$. Yield $70 \%$, yellow solid, m.p. $127-129^{\circ} \mathrm{C}$. IR (KBr) $v_{\max }=2868,1597,1561,1298,1118,903 \mathrm{~cm}^{-1} .{ }^{1} \mathrm{H}$ NMR $\left(400 \mathrm{MHz}, \mathrm{DMSO}-\mathrm{d}_{6}\right) \delta=2.59(6 \mathrm{H}, \mathrm{s}$, $\left.\mathrm{NCH}_{3}\right), 3.96-4.10\left(2 \mathrm{H}, \mathrm{m}, \mathrm{CH}_{2}\right.$ oxa $), 4.52-4.81\left(4 \mathrm{H}, \mathrm{m}, \mathrm{CH}_{2}\right.$ oxa, $\left.\mathrm{NCH}_{2}\right), 7.59(1 \mathrm{H}, \mathrm{d}, J=7.7 \mathrm{~Hz}, \mathrm{H}$ pyr $)$, $7.91(1 \mathrm{H}, \mathrm{d}, J=7.5 \mathrm{~Hz}, \mathrm{H}$ pyr $), 8.41(1 \mathrm{H}, \mathrm{s}, \mathrm{H}$ pyr $) \mathrm{ppm} ;{ }^{13} \mathrm{C}$ NMR $\left(100 \mathrm{MHz}, \mathrm{DMSO}-\mathrm{d}_{6}\right) \delta=42.0\left(\mathrm{CH}_{3}\right)$, $49.3\left(\mathrm{CH}_{2}\right), 50.8\left(\mathrm{CH}_{2}\right), 68.2\left(\mathrm{CH}_{2}\right), 104.0,105.1,124.4(\mathrm{CH}), 130.0,139.8(\mathrm{CH}), 142.1,149.8(\mathrm{CH}), 150.1$,

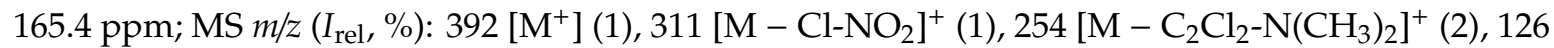
[Cl-pyr-CH $]_{2}^{+}$(100); HRMS (ESI ${ }^{+}$) $m / z$ calcd for $\mathrm{C}_{14} \mathrm{H}_{16} \mathrm{~N}_{4} \mathrm{O}_{3} \mathrm{Cl}_{3}[\mathrm{M}+\mathrm{H}]^{+}$: 393.0283; found: 393.0280.

Synthesis of 1-[(4-bromo-1,3,4-trichloro-2-nitrobuta-1,3-dien-1-yl)sulfanyl]-4-fluorobenzene (13). To a solution of nitrodiene $2(0.316 \mathrm{~g}, 1.0 \mathrm{mmol})$ in dry DCM $(15 \mathrm{~mL})$ at $0{ }^{\circ} \mathrm{C}$, a solution of 4-fluorothiophenole $(0.128$ $\mathrm{g}, 1.0 \mathrm{mmol}$ ) in $5 \mathrm{~mL}$ of dry DCM was added and stirred for $3 \mathrm{~h}$. After reaching r.t. and further stirring for $24 \mathrm{~h}$ the mixture was concentrated and water $(20 \mathrm{~mL})$ was added before extraction with chloroform $(3 \times 10 \mathrm{~mL})$. The product was purified by column chromatography using petroleum ether-ethyl acetate (10:1) and dried in vacuo. A 1:1 mixture of isomers was obtained. Yield $0.301 \mathrm{~g}(74 \%)$, yellow solid, m.p. $98-99^{\circ} \mathrm{C}$. IR (ATR) $v_{\max }=1587,1527,1292,1223,815,526 \mathrm{~cm}^{-1} .{ }^{1} \mathrm{H}$ NMR $\left(400 \mathrm{MHz}, \mathrm{CDCl}_{3}\right) \delta=$ 7.11-7.25 (2H, m, H Ph), 7.48-7.64 (2H, m, H Ph) ppm; $\left.{ }^{13} \mathrm{C} \mathrm{NMR} \mathrm{(101} \mathrm{MHz,} \mathrm{CDCl}_{3}\right) \delta=115.2,116.4$ (CClBr), 117.0, $117.2(\mathrm{CH}), 124.0,124.1,124.2,124.5,138.3,138.4(\mathrm{CH}), 157.3,157.8(\mathrm{CClS}), 163.4,165.9$ (CF) ppm, $\mathrm{CNO}_{2}$ could not be detected; MS m/z (Irel \%): $405\left[\mathrm{M}^{+}\right](1), 370[\mathrm{M}-\mathrm{Cl}]^{+}(1), 326[\mathrm{M}-\mathrm{Br}]^{+}$ (6), $291[\mathrm{M}-\mathrm{Br}-\mathrm{Cl}]^{+}$(3), 127 [S-Ph-F] ${ }^{+}(100)$; $\mathrm{HRMS}\left(\mathrm{ESI}^{+}\right) \mathrm{m} / z$ calcd for $\mathrm{C}_{10} \mathrm{H}_{5} \mathrm{NO}_{2} \mathrm{Cl}_{3} \mathrm{SBrF}[\mathrm{M}+\mathrm{H}]^{+}$: 405.8269; found: 405.8286 .

Synthesis of 5-(((2E)-2-(3-bromo-2,3-dichloro-1-nitroallylidene)imidazolidin-1-yl)methyl)-2-chloropyridine (14) and 1,1'-[(4-bromo-3,4-dichloro-2-nitrobuta-1,3-diene-1,1-diyl)disulfanediyl]bis(4-fluorobenzene) (15). A solution of $\mathrm{N}$-[(6-chloropyridin-3-yl)methyl]ethane-1,2-diamine $10 \mathrm{a}(0.515 \mathrm{~g}, 3.0 \mathrm{mmol})$ in $\mathrm{MeOH}$ 
$(5 \mathrm{~mL})$ was added to a suspension of diene $13(0.407 \mathrm{~g}, 1.0 \mathrm{mmol}$, a $1: 1$ mixture of isomers $)$ in $\mathrm{MeOH}$ $(10 \mathrm{~mL})$ at $-10^{\circ} \mathrm{C}$ and stirred for $1 \mathrm{~h}$ at the same temperature. The precipitated bisthiodiene $\mathbf{1 5}$ was filtered off, washed with water and cold $\mathrm{MeOH}(2 \times 3 \mathrm{~mL})$, and dried under reduced pressure to yield diene 15. The collected filtrates were carefully neutralized by means of hydrochloric acid and stirred with additional $50 \mathrm{~mL}$ of water. Again, the solid was filtered off, and then washed with water and diethyl ether $(3 \times 5 \mathrm{~mL})$. Recrystallization from methanol gave imidazolidine 14 .

Imidazolidine 14. A 1: 1 mixture of isomers was obtained. Yield $0.189 \mathrm{~g}(44 \%)$, white solid, m.p. 172-173 ${ }^{\circ} \mathrm{C}$. IR $(\mathrm{KBr}) v_{\max }=3312,3055,2917,1588,1420,1137,824 \mathrm{~cm}^{-1} .{ }^{1} \mathrm{H}$ NMR $\left(200 \mathrm{MHz}, \mathrm{DMSO}-\mathrm{d}_{6}\right) \delta=$ 3.67-3.90 (4H, m, CH $\mathrm{CH}_{2}$ ), 4.46-4.60 (2H, m, NCH- -pyr), 7.48-7.60 (1H, m, H pyr), 7.71-7.81 (1H, m, H pyr), 8.24-8.39 (1H, m, H pyr), $9.41(1 \mathrm{H}, \mathrm{s}, \mathrm{NH}) \mathrm{ppm} ;{ }^{13} \mathrm{C}$ NMR $\left(50 \mathrm{MHz}, \mathrm{DMSO}-\mathrm{d}_{6}\right) \delta=42.7\left(\mathrm{CH}_{2} \mathrm{imi}\right)$, $49.4\left(\mathrm{NCH}_{2}\right.$-pyr), 50.8 ( $\left.\mathrm{CH}_{2} \mathrm{imi}\right), 103.4,105.2\left(\mathrm{CNO}_{2}\right), 113.0,113.8(\mathrm{CClBr}), 124.3,124.4(\mathrm{CH}), 126.6$, $128.7,131.4,131.5,137.9,138.0(\mathrm{CH}), 147.9,148.0,149.6,159.6,159.7(\mathrm{NCN}) \mathrm{ppm} ; \mathrm{MS} m / z\left(I_{\mathrm{rel}}, \%\right): 426$ $\left[\mathrm{M}^{+}\right](1), 380\left[\mathrm{M}-N O_{2}\right]^{+}(1), 347[\mathrm{M}-\mathrm{Br}]^{+}(20), 314[\mathrm{M}-\mathrm{pyr}]^{+}$(3), $312[\mathrm{M}-\mathrm{Br}-\mathrm{Cl}]^{+}$(3), $254\left[\mathrm{M}-\mathrm{C}_{2} \mathrm{Cl}_{2} \mathrm{Br}+\mathrm{H}\right]^{+}$ (35), 126 [Cl-pyr-CH$]^{+}$(100); $\mathrm{HRMS}\left(\mathrm{ESI}^{+}\right) \mathrm{m} / \mathrm{z}$ calcd for $\mathrm{C}_{12} \mathrm{H}_{9} \mathrm{~N}_{4} \mathrm{O}_{2} \mathrm{Cl}_{3} \mathrm{Br}[\mathrm{M}-\mathrm{H}]^{-}$: 424.8980; found: 424.8967.

Bisthiodiene 15. A 1: 1 mixture of isomers was obtained. Yield $0.150 \mathrm{~g}(30 \%)$, yellow solid, m.p. 141-142 ${ }^{\circ} \mathrm{C}$. IR (ATR) $v_{\max }=1589,1489,1293,1230,823,505 \mathrm{~cm}^{-1} .{ }^{1} \mathrm{H} \mathrm{NMR}\left(400 \mathrm{MHz}, \mathrm{CDCl}_{3}\right) \delta=$ 6.84-7.01 (6H, m, H Ph), 7.05-7.17 (2H, m, H Ph) ppm; ${ }^{13} \mathrm{C} \mathrm{NMR}\left(101 \mathrm{MHz}, \mathrm{CDCl}_{3}\right) \delta=115.1,117.2$ (CClBr), 116.3-116.7 (CH Ph), 123.4, 125.5, 126.1 ( $\left.\mathrm{C}_{\mathrm{Ph}}-\mathrm{S}\right), 133.4,133.5,136.5,136.6,136.7$ (C Ph), 138.3, $138.4\left(\mathrm{CNO}_{2}\right), 159.1,159.5(\mathrm{CSS}), 163.1\left(\mathrm{CF}, J_{\mathrm{C}, \mathrm{F}}=251.5 \mathrm{~Hz}\right), 163.1\left(\mathrm{CF}, J_{\mathrm{C}, \mathrm{F}}=252.3 \mathrm{~Hz}\right) \mathrm{ppm} ; \mathrm{MS}, \mathrm{m} / z\left(I_{\mathrm{rel}}\right.$, \%): $497\left[\mathrm{M}^{+}\right](2), 462[\mathrm{M}-\mathrm{Cl}]^{+}(2), 418[\mathrm{M}-\mathrm{Br}]^{+}(30), 383[\mathrm{M}-\mathrm{Br}-\mathrm{Cl}]^{+}(3), 372\left[\mathrm{M}-\mathrm{Br}_{-} \mathrm{NO}_{2}\right]^{+}(4), 127$ [S-Ph-F] ${ }^{+}(100) ; \mathrm{HRMS}\left(\mathrm{ESI}^{+}\right) \mathrm{m} / z$ calcd for $\mathrm{C}_{16} \mathrm{H}_{9} \mathrm{NO}_{2} \mathrm{BrCl}_{2} \mathrm{~F}_{2} \mathrm{~S}_{2}[\mathrm{M}+\mathrm{H}]^{+}:$497.8598; found 497.8588.

Alternative synthesis of diene 15: To a solution of bromonitrodiene 2 ( $0.316 \mathrm{~g}, 1.0 \mathrm{mmol}$, a 47 : 53 mixture of isomers) and 4-fluorobenzenethiol $(0.256 \mathrm{~g}, 2.0 \mathrm{mmol})$ in $\mathrm{MeOH}(10 \mathrm{~mL})$ at $0{ }^{\circ} \mathrm{C}$, a solution of sodium methanolate $(0.108 \mathrm{~g}, 2.0 \mathrm{mmol})$ in $\mathrm{MeOH}(5 \mathrm{~mL})$ was added. The solution was stirred for $3 \mathrm{~h}$ at $0{ }^{\circ} \mathrm{C}$ and at r.t. for $1 \mathrm{~d}$. Subsequently, the solution was concentrated and the precipitate filtered off and washed with diluted $\mathrm{HCl}(5 \mathrm{~mL})$ and cold $\mathrm{MeOH}(2 \times 2 \mathrm{~mL})$. A mixture of both isomers was obtained. Yield of bisthiodiene 15 is $0.414 \mathrm{~g}$ (83\%, a 1:1 mixture of isomers).

N,N'-Bis[(2-chloro-1,3-thiazol-5-yl)methyl]ethane-1,2-diamine (16) was prepared according to the literature [13] in 70\% yield.

Synthesis of 5,5'-\{[2-(3-bromo-2,3-dichloro-1-nitroprop-2-en-1-ylidene)imidazolidine-1,3-diyl] dimethanediyl\}-bis(2-chloro-1,3-thiazole) (17). To a solution of diene $2(0.166 \mathrm{~g}, 0.5 \mathrm{mmol})$ in a mixture of $\mathrm{MeOH}$ and water $(10 \mathrm{~mL}, 10: 1)$ at $0^{\circ} \mathrm{C} N, N^{\prime}$-bis[(2-chloro-1,3-thiazol-5-yl)methyl]ethane-1,2-diamine (16) $(0.170 \mathrm{~g}, 0.5 \mathrm{mmol})$ and $\mathrm{Na}_{2} \mathrm{CO}_{3}(0.112 \mathrm{~g}, 2.0 \mathrm{mmol})$ were added carefully. After $1 \mathrm{~h}$ at $0{ }^{\circ} \mathrm{C}$, the mixture was allowed to reach r.t. and stirred for another $5 \mathrm{~h}$. Subsequently, the mixture was concentrated in vacuo and the resulting precipitate was filtered off and washed with cold $\mathrm{MeOH}(2 \times 5$ $\mathrm{mL})$, water $(2 \times 5 \mathrm{~mL})$ and again $\mathrm{MeOH}(1 \times 5 \mathrm{~mL})$. A 1: 1 mixture of isomers was obtained. Yield 0.252 $\mathrm{g}(89 \%)$, white solid, $\mathrm{mp} 162-163{ }^{\circ} \mathrm{C}$. IR (ATR) $v_{\max }=1569,1533,1325,1143,1046,770 \mathrm{~cm}^{-1} .{ }^{1} \mathrm{H} \mathrm{NMR}$ $\left(400 \mathrm{MHz}, \mathrm{DMSO}_{6}\right) \delta=3.73-3.96\left(4 \mathrm{H}, \mathrm{m}, \mathrm{CH}_{2}\right), 4.58-4.80\left(4 \mathrm{H}, \mathrm{m}, \mathrm{imi}-\mathrm{CH}_{2}\right), 7.69(2 \mathrm{H}, \mathrm{s}, \mathrm{CH}) \mathrm{ppm}$; ${ }^{13} \mathrm{C}$ NMR $\left(100 \mathrm{MHz}, \mathrm{DMSO}-\mathrm{d}_{6}\right) \delta=45.2,45.4\left(\mathrm{CH}_{2} \mathrm{imi}\right), 45.2,45.4\left(\mathrm{CH}_{2} \mathrm{imi}\right), 47.2,47.3\left(\mathrm{imi}^{-} \mathrm{CH}_{2}\right), 98.1$, $99.7\left(\mathrm{CNO}_{2}\right), 109.7,110.6(\mathrm{CClBr}), 127.0,128.8(\mathrm{CCl}), 133.6,133.7$ (SC thiaz), 141.8, 141.9 (NC thiaz), 151.7, 151.8 (NCS), 161.6, 161.8 (NCN) ppm; MS m/z (I rel $\%$ ): $563\left[\mathrm{M}^{+}\right](1), 527[\mathrm{M}-\mathrm{Cl}]^{+}(1), 484[\mathrm{M}$ $-\mathrm{Br}]^{+}(1), 132$ [thiazole-Cl-CH$]^{+}(100)$; HRMS $\left(\mathrm{ESI}^{+}\right) \mathrm{m} / \mathrm{z}$ calcd for $\mathrm{C}_{14} \mathrm{H}_{11} \mathrm{~N}_{5} \mathrm{O}_{2} \mathrm{Cl}_{4} \mathrm{BrS}_{2}[\mathrm{M}+\mathrm{H}]^{+}$: 563.8286; found: 563.8284 .

Ethyl [(1,3,4,4-tetrachloro-2-nitrobuta-1,3-dien-1-yl)sulfanyl]acetate (18) was prepared according to the literature [18] in $80 \%$ yield. 
Synthesis of 3-[4-(propan-2-yl)phenyl]-2-(2,3,3-trichloro-1-nitroprop-2-en-1-ylidene)-1,3-thiazolidin-4-one (19a) (General method). To a suspension of the acetate $18(0.355 \mathrm{~g}, 1.0 \mathrm{mmol})$ in $\mathrm{MeOH}(3 \mathrm{~mL})$ at $-18{ }^{\circ} \mathrm{C}$ a solution of 4-(propan-2-yl)aniline (0.297 g, $2.2 \mathrm{mmol}$ ) in $\mathrm{MeOH}$ (3 mL) was added dropwise within 10 min. The mixture was stirred for $3 \mathrm{~h}$ at $-18^{\circ} \mathrm{C}$ and $12 \mathrm{~h}$ at r.t. before it was concentrated. The resulting precipitate was filtered off and washed with cold $\mathrm{MeOH}(1 \times 5 \mathrm{~mL})$, water $(2 \times 5 \mathrm{~mL})$ and again $\mathrm{MeOH}$ $(2 \times 5 \mathrm{~mL})$. The product was dried in vacuo. Yield $0.298 \mathrm{~g}(73 \%)$, beige solid, m.p. $208-209{ }^{\circ} \mathrm{C}$. IR (ATR) $v_{\max }=2962,1758,1520,1289,1167,686 \mathrm{~cm}^{-1} .{ }^{1} \mathrm{H}$ NMR $\left(400 \mathrm{MHz}, \mathrm{DMSO}-\mathrm{d}_{6}\right) \delta=1.22(3 \mathrm{H}, \mathrm{d}, J=6.9$ $\mathrm{Hz}, i-\mathrm{Pr}), 1.23(3 \mathrm{H}, \mathrm{d}, J=6.9 \mathrm{~Hz}, i-\mathrm{Pr}), 2.95(1 \mathrm{H}$, sep, $J=6.8 \mathrm{~Hz}, i-\mathrm{Pr}), 4.13\left(1 \mathrm{H}, \mathrm{d}, J=18.7 \mathrm{~Hz}, \mathrm{SCH}_{2}\right)$, $4.17\left(1 \mathrm{H}, \mathrm{d}, J=18.7 \mathrm{~Hz}, \mathrm{SCH}_{2}\right), 7.28-7.33(2 \mathrm{H}, \mathrm{m}, \mathrm{H} \mathrm{Ar}), 7.34-7.40$ (2H, m, H Ar) ppm; ${ }^{13} \mathrm{C} \mathrm{NMR}(100$ MHz, DMSO-d 6$) \delta=23.6,23.8\left(\mathrm{CH}_{3}\right), 32.5\left(\mathrm{CH}_{2}\right), 33.6(\mathrm{CH} i-\mathrm{Pr}), 121.3\left(\mathrm{CCl}_{2}\right), 121.4\left(\mathrm{CNO}_{2}\right), 126.9$ (CH), $127.3(\mathrm{CH}), 127.4(\mathrm{CH}), 128.4,128.8(\mathrm{CH}), 132.1,150.6,165.8(\mathrm{NCS}), 174.1(\mathrm{C}=\mathrm{O}) \mathrm{ppm}$; MS m/z $\left(I_{\text {rel }}, \%\right): 406\left[\mathrm{M}^{+}\right](10), 389[\mathrm{M}-\mathrm{OH}]^{+}(5), 371[\mathrm{M}-\mathrm{Cl}]^{+}(5), 278\left[\mathrm{M}-\mathrm{C}_{2} \mathrm{Cl}_{3}+\mathrm{H}\right]^{+}(15), 261\left[\mathrm{M}-\mathrm{C}_{2} \mathrm{Cl}_{3}-\mathrm{O}\right]^{+}$ (100); $\mathrm{MS}\left(\mathrm{ESI}^{-}\right) \mathrm{m} / \mathrm{z}$ calcd for $\mathrm{C}_{15} \mathrm{H}_{12} \mathrm{~N}_{2} \mathrm{O}_{3} \mathrm{Cl}_{3} \mathrm{~S}$ 405.0; found: 405.0 .

2-(2,3,3-Trichloro-1-nitroprop-2-en-1-ylidene)-3-[4-(trifluoromethyl)phenyl]-1,3-thiazolidin-4-one (19b). Same procedure as for 19a, using 4-(trifluoromethyl)aniline $(0.355 \mathrm{~g}, 2.2 \mathrm{mmol})$ at $0{ }^{\circ} \mathrm{C}$ for $3 \mathrm{~h}$ and $15 \mathrm{~h}$ at r.t.. Yield $0.330 \mathrm{~g}(76 \%)$, yellowish solid, m.p. 228-229 ${ }^{\circ} \mathrm{C}$. IR (ATR) $v_{\max }=3058,1737,1515,1285,1170$, $691 \mathrm{~cm}^{-1} .{ }^{1} \mathrm{H}$ NMR $\left(400 \mathrm{MHz}, \mathrm{DMSO}-\mathrm{d}_{6}\right) \delta=4.13\left(1 \mathrm{H}, \mathrm{d}, J=18.7 \mathrm{~Hz}, \mathrm{SCH}_{2}\right), 4.20(1 \mathrm{H}, \mathrm{d}, J=18.7 \mathrm{~Hz}$, $\left.\mathrm{SCH}_{2}\right), 7.67-7.74(2 \mathrm{H}, \mathrm{m}, \mathrm{H} \mathrm{Ar}), 7.91-7.98(2 \mathrm{H}, \mathrm{m}, \mathrm{H} \mathrm{Ar}) \mathrm{ppm} ;{ }^{13} \mathrm{C} \mathrm{NMR}\left(400 \mathrm{MHz}, \mathrm{DMSO}-\mathrm{d}_{6}\right) \delta=32.8$ $\left(\mathrm{CH}_{2}\right), 121.2\left(\mathrm{CNO}_{2}\right), 121.3\left(\mathrm{CCl}_{2}\right), 123.9\left(J_{\mathrm{C}, \mathrm{F}}=272.4 \mathrm{~Hz}, \mathrm{CF}_{3}\right), 126.2\left(J_{\mathrm{C}, \mathrm{F}}=3.9 \mathrm{~Hz}, \mathrm{CH}\right), 126.6\left(J_{\mathrm{C}, \mathrm{F}}=\right.$ $4.2 \mathrm{~Hz}, \mathrm{CH}), 128.9(\mathrm{CH}), 129.1,130.2(\mathrm{CH}), 130.7\left(J_{\mathrm{C}, \mathrm{F}}=32.3 \mathrm{~Hz}, \mathrm{CF}_{3}-\mathrm{C}\right), 138.1,165.4(\mathrm{NCS}), 173.9(\mathrm{C}=\mathrm{O})$

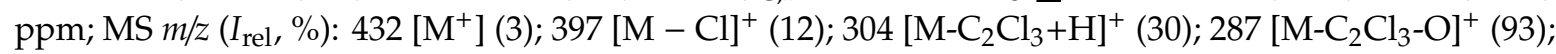
145 (100); HRMS (ESI ${ }^{-}$) m/z calcd for $\mathrm{C}_{13} \mathrm{H}_{5} \mathrm{~N}_{2} \mathrm{O}_{3} \mathrm{Cl}_{3} \mathrm{SF}_{3}[\mathrm{M}-\mathrm{H}]^{-}$: 430.9044; found: 430.9062.

Synthesis of 3-(hydrazinylidenemethyl)-4-nitro-N-[4-(propan-2-yl)phenyl]-1H-pyrazol-5-amine (20a). To a stirred suspension of thiazolidinone 19a $(0.408 \mathrm{~g}, 1.0 \mathrm{mmol})$ in $\mathrm{MeOH}(10 \mathrm{~mL})$ at $-18{ }^{\circ} \mathrm{C}$, a solution of hydrazine hydrate $(0.400 \mathrm{~g}, 8.0 \mathrm{mmol})$ in $\mathrm{MeOH}(5 \mathrm{~mL})$ was added dropwise within $5 \mathrm{~min}$. Subsequently, the mixture was allowed to reach $0{ }^{\circ} \mathrm{C}$. After $5 \mathrm{~h}$, and $18 \mathrm{~h}$ at r.t., the solution was concentrated and neutralized to $\mathrm{pH} 7$ with $\mathrm{HCl}(5 \%)$, then extracted with ethyl acetate $(3 \times 10 \mathrm{~mL})$. The combined organic phases were purified over a short column chromatography using ethyl acetate-petroleum ether (1:1). Subsequently, the obtained solution was concentrated and the resulting precipitate was filtered and washed with diethyl ether $(2 \times 3 \mathrm{~mL})$. Yield $0.150 \mathrm{~g}(52 \%)$, red solid, m.p. $194-195^{\circ} \mathrm{C}$. IR $($ ATR $) v_{\max }=$ $3365,2956,1597,1562,1461,1350,579 \mathrm{~cm}^{-1} .{ }^{1} \mathrm{H}$ NMR $\left(400 \mathrm{MHz}, \mathrm{DMSO}-\mathrm{d}_{6}\right) \delta=1.18(6 \mathrm{H}, \mathrm{d}, J=6.9 \mathrm{~Hz}$, $\mathrm{CH}_{3}$ i-Pr), $2.83(1 \mathrm{H}$, sep, $J=6.8 \mathrm{~Hz}, \mathrm{CH}$-Pr $), 7.16(2 \mathrm{H}, \mathrm{d}, J=8.5 \mathrm{~Hz}, \mathrm{H} \mathrm{Ar}), 7.61(2 \mathrm{H}, \mathrm{d}, J=8.4 \mathrm{~Hz}, \mathrm{H}$ Ar), $8.07(1 \mathrm{H}, \mathrm{s}, \mathrm{NCH}), 8.15\left(2 \mathrm{H}, \mathrm{s}, \mathrm{N}-\mathrm{NH}_{2}\right), 8.53\left(1 \mathrm{H}, \mathrm{s}, \mathrm{NH}\right.$ anil), $13.29(1 \mathrm{H}, \mathrm{s}, \mathrm{NH}$ pyr $)$ ppm; ${ }^{13} \mathrm{C} \mathrm{NMR}$ $\left(100 \mathrm{MHz}, \mathrm{DMSO}-\mathrm{d}_{6}\right) \delta=24.2\left(\mathrm{CH}_{3}\right), 32.9(\mathrm{CH} i$-Pr $), 117.4\left(\mathrm{CNO}_{2}\right), 118.0(2 \times \mathrm{CH} \mathrm{Ph}), 122.7(\mathrm{NCH})$, $126.7(2 \times \mathrm{CH} \mathrm{Ph}), 138.3,138.7,141.4,147.4(\mathrm{NNHC}) \mathrm{ppm} ; \mathrm{MS} m / z\left(I_{\mathrm{rel}}, \%\right): 288\left[\mathrm{M}^{+}\right](60), 273$ [M $\left.\mathrm{CH}_{3}\right]^{+}(100), 256$ [M-CH$\left._{3}-\mathrm{OH}\right]^{+}$(5), $245[\mathrm{M}-i-\mathrm{Pr}]^{+}$(8), 169 [M-NH-Ph-i-Pr] $^{+}$(5); HRMS (ESI ${ }^{-}$) m/z calcd for $\mathrm{C}_{13} \mathrm{H}_{15} \mathrm{~N}_{6} \mathrm{O}_{2}[\mathrm{M}-\mathrm{H}]^{-}$: 287.1261; found: 287.1272 .

Synthesis of 3-(hydrazinylidenemethyl)-4-nitro-N-[4-(trifluoromethyl)phenyl]-1H-pyrazol-5-amine (20b). To a stirred suspension of thiazolidinone $19 \mathrm{~b}(0.434 \mathrm{~g}, 1.0 \mathrm{mmol})$ in $\mathrm{MeOH}(10 \mathrm{~mL})$ at $-18^{\circ} \mathrm{C}$, a solution of hydrazine hydrate $(0.250 \mathrm{~g}, 5.0 \mathrm{mmol})$ in $\mathrm{MeOH}(5 \mathrm{~mL})$ was added dropwise within $5 \mathrm{~min}$. Subsequently, the mixture was allowed to reach $0{ }^{\circ} \mathrm{C}$. After $5 \mathrm{~h}$ with stirring at $0{ }^{\circ} \mathrm{C}$ and $18 \mathrm{~h}$ at r.t., the solution was concentrated and the resulting precipitate filtered off. Washing the product with cold $\mathrm{MeOH}(2 \times 5$ $\mathrm{mL}$ ) and drying in vacuo yielded the pyrazole 20b. Yield $0.145 \mathrm{~g}(46 \%)$, yellow solid, m.p. 248-250 ${ }^{\circ} \mathrm{C}$. IR (ATR) $v_{\max }=3440,1602,1516,1327,1160,832 \mathrm{~cm}^{-1} .{ }^{1} \mathrm{H}$ NMR $\left(400 \mathrm{MHz}, \mathrm{DMSO}-\mathrm{d}_{6}\right) \delta=7.63$ $(2 \mathrm{H}, \mathrm{d}, J=8.7 \mathrm{~Hz}, \mathrm{H} \mathrm{Ph}), 7.91(2 \mathrm{H}, \mathrm{d}, J=8.6 \mathrm{~Hz}, \mathrm{H} \mathrm{Ph}), 8.07(1 \mathrm{H}, \mathrm{s}, \mathrm{NCH}), 8.21\left(2 \mathrm{H}, \mathrm{s}, \mathrm{NH}_{2}\right), 8.97(1 \mathrm{H}$, s, Ph-NH), 13.45 (1H, s, NH Pyr) ppm; ${ }^{13} \mathrm{C}$ NMR (100 MHz, DMSO-d 6 ) $\delta=117.6$ (2× CH Ph), 117.8 $\left(\mathrm{CNO}_{2}\right), 121.0\left(J_{\mathrm{C}, \mathrm{F}}=32.1 \mathrm{~Hz}, \mathrm{CCF}_{3}\right), 122.2\left(J_{\mathrm{C}, \mathrm{F}}=270.7 \mathrm{~Hz}, \mathrm{CF}_{3}\right), 122.5(\mathrm{NCH}), 126.2\left(J_{\mathrm{C}, \mathrm{F}}=3.7 \mathrm{~Hz}\right.$, $2 \times \mathrm{CH}$ Ph), 139.0, 144.2, $146.4(\mathrm{NNHC})$ ppm;. MS m/z (I rel $\%): 314\left[\mathrm{M}^{+}\right](2), 270\left[\mathrm{M}_{-} \mathrm{CH}_{2}=\mathrm{N}-\mathrm{NH}_{2}\right]^{+}$ 
(3), $254\left[\mathrm{M}-\mathrm{CH}_{2}=\mathrm{N}-\mathrm{NH}_{2}-\mathrm{O}\right]^{+}(14), 161\left[\mathrm{CF}_{3}-\mathrm{Ph}-\mathrm{NH}_{2}\right]^{+}$(60), $142\left[\mathrm{CF}_{2}-\mathrm{Ph}-\mathrm{NH}_{2}\right]^{+}$(27), 96 (100); HRMS (ESI ${ }^{-}$) $\mathrm{m} / \mathrm{z}$ calcd for $\mathrm{C}_{11} \mathrm{H}_{8} \mathrm{~N}_{6} \mathrm{O}_{2} \mathrm{~F}_{3}[\mathrm{M}-\mathrm{H}]^{-}$: 313.0665; found: 313.0666 .

Synthesis of 5-(furan-2-ylmethylidene)-3-[4-(propan-2-yl)phenyl]-2-(2,3,3-trichloro-1-nitroprop-2-en1-ylidene)-1,3-thiazolidin-4-one (21a) (General method). To a suspension of thiazolidinone 19a (0.408 g, 1.0 $\mathrm{mmol})$ in acetic acid $(15 \mathrm{~mL})$ furan-2-carbaldehyde $(0.115 \mathrm{~g}, 1.2 \mathrm{mmol})$ and triethylamine $(0.152 \mathrm{~g}, 1.5$ $\mathrm{mmol}$ ) were added. The resulting mixture was refluxed for $4 \mathrm{~h}$. After concentration of this mixture in vacuo to a volume of about $3 \mathrm{~mL}$ and cooling to r.t., the resulting precipitate was filtered off and washed with water $(2 \times 10 \mathrm{~mL})$ and cold $\mathrm{MeOH}(1 \times 2 \mathrm{~mL})$. The product was dried in vacuo. Yield $0.466 \mathrm{~g}(96 \%)$, orange solid, m.p. $228-229^{\circ} \mathrm{C}$. IR (ATR) $v_{\max }=2958,1718,1598,1519,1167,764 \mathrm{~cm}^{-1}$. ${ }^{1} \mathrm{H}$ NMR $\left(400 \mathrm{MHz}, \mathrm{DMSO}_{-} \mathrm{d}_{6}\right) \delta=1.24\left(3 \mathrm{H}, \mathrm{d}, J=6.9 \mathrm{~Hz}, \mathrm{CH}_{3} i-\mathrm{Pr}\right), 1.25\left(3 \mathrm{H}, \mathrm{d}, J=6.9 \mathrm{~Hz}, \mathrm{CH}_{3} i-\mathrm{Pr}\right)$, $2.97(1 \mathrm{H}$, sep, $J=6.8 \mathrm{~Hz}, \mathrm{CH} i-\mathrm{Pr}), 6.86(1 \mathrm{H}, \mathrm{dd}, J=1.8 \mathrm{~Hz}, J=3.6 \mathrm{~Hz}, \mathrm{H}$ fur $), 7.34(1 \mathrm{H}, \mathrm{d}, J=3.5 \mathrm{~Hz}, \mathrm{H}$ fur), 7.37-7.45 $(4 \mathrm{H}, \mathrm{m}, \mathrm{H} \mathrm{Ar}), 7.90(1 \mathrm{H}, \mathrm{s},=\mathrm{CH}), 8.30(1 \mathrm{H}, \mathrm{d}, J=1.7 \mathrm{~Hz}, \mathrm{H}$ fur $) \mathrm{ppm} ;{ }^{13} \mathrm{C}$ NMR $(100$ MHz, DMSO-d $\left.\mathrm{d}_{6}\right) \delta=23.6\left(\mathrm{CH}_{3}\right), 23.9\left(\mathrm{CH}_{3}\right), 33.6\left(\mathrm{CH} i\right.$-Pr), $114.4(\mathrm{CH}), 116.7,120.8\left(\mathrm{CNO}_{2}\right), 121.0$

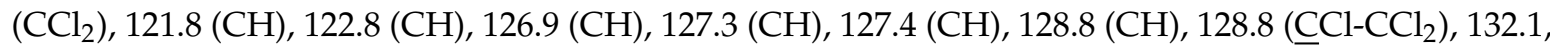
$149.4(\mathrm{CH}), 149.9,150.8,158.5(\mathrm{NCS}), 166.5(\mathrm{C}=\mathrm{O}) \mathrm{ppm}$; MS m/z (I $\left.\mathrm{rel}_{\mathrm{rel}} \%\right): 484$ [M $\left.^{+}\right](6), 449[\mathrm{M}-\mathrm{Cl}]^{+}(2)$, $339\left[\mathrm{M}-\mathrm{C}_{2} \mathrm{Cl}_{3}-\mathrm{O}\right]^{+}(75), 325$ [M-furan-CH=C=S-Cl]$]^{+}(7), 124$ [furan-CH=C=S] ${ }^{+}(100)$. HRMS $\left(\mathrm{ESI}^{+}\right) \mathrm{m} / \mathrm{z}$ calcd for $\mathrm{C}_{20} \mathrm{H}_{15} \mathrm{~N}_{2} \mathrm{O}_{4} \mathrm{SCl}_{3} \mathrm{Na}[\mathrm{M}+\mathrm{Na}]^{+}$: 506.9716; found: 506.9712 .

3-[4-(Propan-2-yl)phenyl]-5-(thiophen-2-ylmethylidene)-2-(2,3,3-trichloro-1-nitroprop-2-en-1-ylidene) -1,3-thiazolidin-4-one (21b). Same procedure as for 21a, using thiophene-2-carbaldehyde (0.135 g, 1.2 $\mathrm{mmol})$. After filtration of the product, it was further purified by column chromatography using chloroform. Yield $0.462 \mathrm{~g}(92 \%)$, dark yellow solid, m.p. $221-223^{\circ} \mathrm{C}$. IR (ATR) $v_{\max }=2963,1717,1586$, $1525,1258,729 \mathrm{~cm}^{-1} .{ }^{1} \mathrm{H}$ NMR $\left(400 \mathrm{MHz}, \mathrm{CDCl}_{3}\right) \delta=1.29\left(6 \mathrm{H}, \mathrm{d}, J=6.7 \mathrm{~Hz}, \mathrm{CH}_{3} i-\mathrm{Pr}\right), 2.99(1 \mathrm{H}$, sep, $J=6.9 \mathrm{~Hz}, \mathrm{CH} i-\mathrm{Pr}), 7.16-7.20(1 \mathrm{H}, \mathrm{m}, \mathrm{H} \mathrm{Ar}), 7.24-7.27(1 \mathrm{H}, \mathrm{m}, \mathrm{H}$ thien), 7.27-7.30 (1H, m, H Ar), 7.35-7.39 (2H, m, H Ar), $7.56(1 \mathrm{H}, \mathrm{d}, J=3.7 \mathrm{~Hz}, \mathrm{H}$ thien), $7.78(1 \mathrm{H}, \mathrm{d}, J=5.0 \mathrm{~Hz}, \mathrm{H}$ thien), $8.12(1 \mathrm{H}, \mathrm{s}$, $=\mathrm{CH}) \mathrm{ppm} ;{ }^{13} \mathrm{C} \mathrm{NMR}\left(100 \mathrm{MHz}, \mathrm{CDCl}_{3}\right) \delta=23.5\left(\mathrm{CH}_{3}\right), 23.7\left(\mathrm{CH}_{3}\right), 34.1(\mathrm{CH} i-\mathrm{Pr}), 117.6,120.6\left(\mathrm{CCl}_{2}\right)$, 122.6 $\left(\mathrm{CNO}_{2}\right), 126.8(\mathrm{CH}), 127.4(\mathrm{CH}), 127.8(\mathrm{CH}), 127.9(\mathrm{CH}), 129.1(\mathrm{CH}), 129.6(\mathrm{CH}), 129.7\left(\mathrm{CClCCl}_{2}\right)$, 131.6, $133.6(\mathrm{CH}), 134.8(\mathrm{CH}), 137.6,151.4(i-\mathrm{Pr}-\mathrm{C}), 155.3(\mathrm{NCS}), 166.7$ (C=O) ppm; MS m/z ( $\left.I_{\text {rel }}, \%\right): 500$

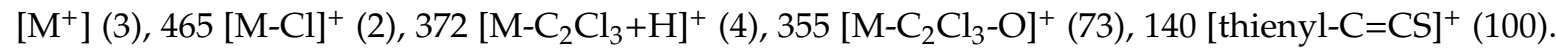
HRMS $\left(\mathrm{ESI}^{+}\right.$) $\mathrm{m} / \mathrm{z}$ calcd for $\mathrm{C}_{20} \mathrm{H}_{15} \mathrm{~N}_{2} \mathrm{O}_{3} \mathrm{Cl}_{3} \mathrm{~S}_{2} \mathrm{Na}[\mathrm{M}+\mathrm{Na}]^{+}$: 522.9487; found: 522.9482.

5-[(1-Methyl-1H-pyrrol-2-yl)methylidene]-3-[4-(propan-2-yl)phenyl]-2-(2,3,3-trichloro-1-nitroprop-2 -en-1-ylidene)-1,3-thiazolidin-4-one (21c). Same procedure as for 21a, using 1-methyl-1H-pyrrole-2-carbaldehyde $(0.328 \mathrm{~g}, 3.0 \mathrm{mmol})$ and triethylamine $(0.304 \mathrm{~g}, 3.0 \mathrm{mmol})$. The mixture was refluxed for $6 \mathrm{~h}$. Yield $0.459 \mathrm{~g}(92 \%)$, red solid, m.p. $244-246{ }^{\circ} \mathrm{C}$. IR (ATR) $v_{\max }=2986$, $1700,1584,1520,1273,745 \mathrm{~cm}^{-1} .{ }^{1} \mathrm{H}$ NMR $\left(400 \mathrm{MHz}, \mathrm{DMSO}_{-} \mathrm{d}_{6}\right) \delta=1.24\left(3 \mathrm{H}, \mathrm{d}, \mathrm{J}=6.9 \mathrm{~Hz}, \mathrm{CH}_{3} \mathrm{i}-\mathrm{Pr}\right)$, $1.25\left(3 \mathrm{H}, \mathrm{d}, \mathrm{J}=6.9 \mathrm{~Hz}, \mathrm{CH}_{3} \mathrm{i}-\mathrm{Pr}\right), 2.98\left(1 \mathrm{H}, \mathrm{sep}, \mathrm{J}=6.9 \mathrm{~Hz}, \mathrm{CH}\right.$ i-Pr), $3.83\left(3 \mathrm{H}, \mathrm{s}, \mathrm{NCH}_{3}\right), 6.47(1 \mathrm{H}, \mathrm{dd}, \mathrm{J}=$ $2.6 \mathrm{~Hz}, \mathrm{~J}=4.2 \mathrm{~Hz}, \mathrm{H}$ pyrr), 6.88 (1H, dd, J = $1.1 \mathrm{~Hz}, \mathrm{~J}=4.2 \mathrm{~Hz}, \mathrm{H}$ pyrr), 7.36-7.44 (5H, m, 4H Ph, H pyrr), $7.84(1 \mathrm{H}, \mathrm{s},=\mathrm{CH}) \mathrm{ppm} ;{ }^{13} \mathrm{C}$ NMR $\left(100 \mathrm{MHz}, \mathrm{DMSO}-\mathrm{d}_{6}\right) \delta=23.6\left(\mathrm{CH}_{3}\right), 23.9\left(\mathrm{CH}_{3} \mathrm{i}-\mathrm{Pr}\right), 33.6\left(\mathrm{NCH}_{3}\right)$, $34.3\left(\mathrm{CH}\right.$ i-Pr), $111.5\left(\mathrm{CCl}_{2}\right), 111.9(\mathrm{CH}), 118.0(\mathrm{CH}), 120.2\left(\mathrm{CNO}_{2}\right), 121.2,124.9(\mathrm{CH}), 126.9(\mathrm{CH}), 127.4$ $(2 \times \mathrm{CH}), 128.0,128.6,128.7(\mathrm{CH}), 131.8(\mathrm{CH}), 132.2,150.7(\mathrm{i}-\mathrm{Pr}-\mathrm{C}), 157.5(\mathrm{NCS}), 166.5(\mathrm{C}=\mathrm{O}) \mathrm{ppm}$; MS $\mathrm{m} / \mathrm{z}\left(\mathrm{I}_{\mathrm{rel}}, \%\right): 497\left[\mathrm{M}^{+}\right](10), 462[\mathrm{M}-\mathrm{Cl}]^{+}(1), 361$ [M-pyrrol-CH=C=S+H]$]^{+}(3), 352\left[\mathrm{M}_{-} \mathrm{C}_{2} \mathrm{Cl}_{3}-\mathrm{CH}_{4}\right]^{+}(25)$, 137 [Me-pyrrol-CH=C=S] ${ }^{+}(100) ; \mathrm{HRMS}\left(\mathrm{ESI}^{+}\right) \mathrm{m} / \mathrm{z}$ calcd for $\mathrm{C}_{21} \mathrm{H}_{18} \mathrm{~N}_{3} \mathrm{O}_{3} \mathrm{Cl}_{3} \mathrm{SNa}[\mathrm{M}+\mathrm{Na}]^{+}: 520.0032$; found: 520.0018 .

\section{5-(Furan-2-ylmethylidene)-2-(2,3,3-trichloro-1-nitroprop-2-en-1-ylidene)-3-[4-(trifluoromethyl)} phenyl]-1,3-thiazolidin-4-one (21d). Same procedure as for 21a, using thiazolidinone $\mathbf{2 1 b}(0.433$ $\mathrm{g}, 1.0 \mathrm{mmol})$, furan-2-carbaldehyde $(0.115 \mathrm{~g}, 1.2 \mathrm{mmol})$ and triethylamine $(0.152 \mathrm{~g}, 1.5 \mathrm{mmol})$. Yield $0.374 \mathrm{~g}(73 \%)$, orange solid, m.p. $227-229^{\circ} \mathrm{C}$. IR (ATR) $v_{\max }=3130,1720,1525,1286,1162,622 \mathrm{~cm}^{-1}$. ${ }^{1} \mathrm{H}$ NMR $\left(400 \mathrm{MHz}, \mathrm{CDCl}_{3}\right) \delta=6.67(1 \mathrm{H}, \mathrm{dd}, \mathrm{J}=1.8 \mathrm{~Hz}, \mathrm{~J}=3.6 \mathrm{~Hz}, \mathrm{H}$ fur $), 6.98(1 \mathrm{H}, \mathrm{d}, \mathrm{J}=3.6 \mathrm{~Hz}, \mathrm{H}$ fur), 7.41-7.45 (1H, m, H Ar), 7.50-7.55 (1H, m, H Ar), $7.72(1 \mathrm{H}, \mathrm{s},=\mathrm{CH}), 7.78-7.84(2 \mathrm{H}, \mathrm{m}, \mathrm{H} \mathrm{Ar}), 7.85$ 
$(1 \mathrm{H}, \mathrm{d}, \mathrm{J}=1.8 \mathrm{~Hz}, \mathrm{H}$ fur $) \mathrm{ppm} ;{ }^{13} \mathrm{C}$ NMR $\left(100 \mathrm{MHz}, \mathrm{CDCl}_{3}\right) \delta=113.9(\mathrm{CH}), 116.9,120.4(\mathrm{CH}), 120.6$ $\left(\mathrm{CCl}_{2}\right), 123.4\left(\mathrm{~J}_{\mathrm{C}, \mathrm{F}}=272.8 \mathrm{~Hz}, \mathrm{CF}_{3}\right), 122.5\left(\mathrm{CNO}_{2}\right), 122.9(\mathrm{CH}), 126.4\left(\mathrm{~J}_{\mathrm{C}, \mathrm{F}}=3.7 \mathrm{~Hz}, \mathrm{CH}\right), 126.9\left(\mathrm{~J}_{\mathrm{C}, \mathrm{F}}=\right.$ $3.7 \mathrm{~Hz}, \mathrm{CH}), 127.8(\mathrm{CH}), 128.8(\mathrm{CH}), 130.2\left(\mathrm{CClCCl}_{2}\right), 132.6\left(\mathrm{~J}_{\mathrm{C}, \mathrm{F}}=33.0 \mathrm{~Hz}, \mathrm{CF}_{3} \underline{\mathrm{C}}\right), 137.2,148.0(\mathrm{CH})$, 149.9, 156.0, $166.4(\mathrm{C}=\mathrm{O}) \mathrm{ppm}$; MS m/z (I $\left.\mathrm{I}_{\mathrm{rel}}, \%\right): 510\left[\mathrm{M}^{+}\right]$(3), $475[\mathrm{M}-\mathrm{Cl}]^{+}(1), 429\left[\mathrm{M}-\mathrm{Cl}^{-\mathrm{NO}_{2}}\right]^{+}(1), 382$

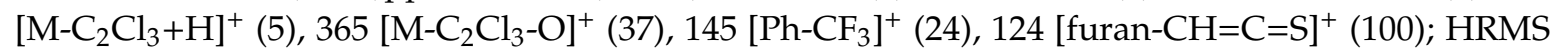
$\left(\mathrm{ESI}^{+}\right) \mathrm{m} / \mathrm{z}$ calcd for $\mathrm{C}_{18} \mathrm{H}_{8} \mathrm{~N}_{2} \mathrm{O}_{4} \mathrm{Cl}_{3} \mathrm{SF}_{3} \mathrm{Na}[\mathrm{M}+\mathrm{Na}]^{+}:$532.9120; found: 532.9117 .

5-(Thiophen-2-ylmethylidene)-2-(2,3,3-trichloro-1-nitroprop-2-en-1-ylidene)-3-[4-(trifluoromethyl)phenyl]-1,3-thiazolidin-4-one (21e). Same procedure as for 21a, using thiazolidinone $\mathbf{2 1 b}(0.433$ $\mathrm{g}, 1.0 \mathrm{mmol})$, thiophene-2-carbaldehyde $(0.135 \mathrm{~g}, 1.2 \mathrm{mmol})$ and triethylamine $(0.152 \mathrm{~g}, 1.5 \mathrm{mmol})$. After filtration, the product was purified by column chromatography using chloroform. The fraction containing the product was dried, suspended in $\mathrm{MeOH}(2 \mathrm{~mL})$, filtered, and the solid washed with cold $\mathrm{MeOH}(1 \times 2 \mathrm{~mL})$. Yield $0.449 \mathrm{~g}(85 \%)$, orange solid, m.p. $245-246{ }^{\circ} \mathrm{C}$. IR (ATR) $v_{\max }=3076,1699$, $1522,1281,1126,730 \mathrm{~cm}^{-1} .{ }^{1} \mathrm{H}$ NMR $\left(400 \mathrm{MHz}, \mathrm{CDCl}_{3}\right) \delta=7.28(1 \mathrm{H}, \mathrm{dd}, \mathrm{J}=3.8 \mathrm{~Hz}, \mathrm{~J}=5.0 \mathrm{~Hz}, \mathrm{H}$ thien), 7.43-7.45 $(1 \mathrm{H}, \mathrm{m}, \mathrm{H} \mathrm{Ar}), 7.52-7.54(1 \mathrm{H}, \mathrm{m}, \mathrm{H} \mathrm{Ar}), 7.58(1 \mathrm{H}, \mathrm{d}, \mathrm{J}=3.7 \mathrm{~Hz}, \mathrm{H}$ thien), 7.79-7.84 (2H, $\mathrm{m}, \mathrm{H} \mathrm{Ar}), 7.84-7.86(1 \mathrm{H}, \mathrm{m}, \mathrm{H}$ thien $), 8.15(1 \mathrm{H}, \mathrm{s},=\mathrm{CH}) \mathrm{ppm} ;{ }^{13} \mathrm{C} \mathrm{NMR}\left(100 \mathrm{MHz}, \mathrm{CDCl}_{3}\right) \delta=116.9$, $120.4\left(\mathrm{CCl}_{2}\right), 123.3\left(\mathrm{~J}_{\mathrm{C}, \mathrm{F}}=271.9 \mathrm{~Hz}, \mathrm{CF}_{3}\right), 126.4\left(\mathrm{~J}_{\mathrm{C}, \mathrm{F}}=3.9 \mathrm{~Hz}, \mathrm{CH}\right), 126.9\left(\mathrm{~J}_{\mathrm{C}, \mathrm{F}}=4.2 \mathrm{~Hz}, \mathrm{CH}\right), 127.8$ $(\mathrm{CH}), 128.8(\mathrm{CH}), 129.3(\mathrm{CH}), 130.3(\mathrm{CH}), 130.4\left(\mathrm{CClCCl}_{2}\right), 132.3\left(\mathrm{~J}_{\mathrm{C}, \mathrm{F}}=32.0 \mathrm{~Hz}, \mathrm{CF}_{3}-\underline{\mathrm{C}}\right), 134.1(\mathrm{CH})$, $135.3(\mathrm{CH}), 137.2,137.4,154.5(\mathrm{NCS}), 166.2(\mathrm{C}=\mathrm{O}) \mathrm{ppm}, \mathrm{CNO}_{2}$ could not be detected; $\mathrm{MS} \mathrm{m} / z\left(\mathrm{I}_{\text {rel }}, \%\right)$ :

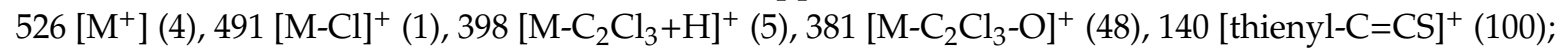
HRMS $\left(\mathrm{ESI}^{+}\right) \mathrm{m} / \mathrm{z}$ calcd for $\mathrm{C}_{18} \mathrm{H}_{8} \mathrm{~N}_{2} \mathrm{O}_{3} \mathrm{Cl}_{3} \mathrm{~S}_{2} \mathrm{~F}_{3} \mathrm{Na}[\mathrm{M}+\mathrm{Na}]^{+}: 548.8892$; found, $\mathrm{m} / z$ : 548.8888 .

5-[(1-Methyl-1H-pyrrol-2-yl)methylidene]-2-(2,3,3-trichloro-1-nitroprop-2-en-1-ylidene)-3- [4-(tri fluoro-methyl)-phenyl]-1,3-thiazolidin-4-one (21f). Same procedure as for 21a, using thiazolidinone $\mathbf{2 1 b}$ $(0.433 \mathrm{~g}, 1.0 \mathrm{mmol}), 1$-methyl-1H-pyrrole-2-carbaldehyde $(0.262 \mathrm{~g}, 2.4 \mathrm{mmol})$ and triethylamine $(0.152$ $\mathrm{g}, 1.5 \mathrm{mmol})$. The mixture was refluxed for $8 \mathrm{~h}$. Yield $0.289 \mathrm{~g}(55 \%)$, red solid, shows fluorescence, m.p. $241-242{ }^{\circ} \mathrm{C}$. IR (ATR) $v_{\max }=1688,1589,1521,1269,1064,620 \mathrm{~cm}^{-1} .{ }^{1} \mathrm{H}$ NMR $(400 \mathrm{MHz}$, DMSO-d 6$) \delta$ $=3.84\left(3 \mathrm{H}, \mathrm{s}, \mathrm{NCH}_{3}\right), 6.48(1 \mathrm{H}, \mathrm{dd}, \mathrm{J}=2.3 \mathrm{~Hz}, \mathrm{~J}=4.1 \mathrm{~Hz}, \mathrm{H}$ pyrr $), 6.90(1 \mathrm{H}, \mathrm{dd}, \mathrm{J}=1.3 \mathrm{~Hz}, \mathrm{~J}=4.1 \mathrm{~Hz}, \mathrm{H}$ pyrr), $7.39(1 \mathrm{H}, \mathrm{dd}, \mathrm{J}=1.3 \mathrm{~Hz}, \mathrm{~J}=2.3 \mathrm{~Hz}, \mathrm{H}$ pyrr $), 7.78-7.86(2 \mathrm{H}, \mathrm{m}, \mathrm{H} \mathrm{Ar}), 7.87(1 \mathrm{H}, \mathrm{s},=\mathrm{CH}), 7.94-8.00$ $(2 \mathrm{H}, \mathrm{m}, \mathrm{H} \mathrm{Ph}) \mathrm{ppm} ;{ }^{13} \mathrm{C}$ NMR $\left(100 \mathrm{MHz}, \mathrm{DMSO}_{-} \mathrm{d}_{6}\right) \delta=34.3\left(\mathrm{NCH}_{3}\right), 111.3,112.1(\mathrm{CH}), 118.2(\mathrm{CH})$, $120.0\left(\mathrm{CNO}_{2}\right), 121.2\left(\mathrm{CCl}_{2}\right), 123.9\left(\mathrm{~J}_{\mathrm{C}, \mathrm{F}}=273.1 \mathrm{~Hz}, \mathrm{CF}_{3}\right), 125.1(\mathrm{CH}), 126.2(\mathrm{CH}), 126.7(\mathrm{CH}), 128.0,129.0$ $(\mathrm{CH}), 129.2,130.1(\mathrm{CH}), 130.8\left(\mathrm{~J}_{\mathrm{C}, \mathrm{F}}=32.3 \mathrm{~Hz}, \mathrm{CCF}_{3}\right), 132.0(\mathrm{CH}), 138.2,157.2(\mathrm{NCS}), 166.2(\mathrm{C}=\mathrm{O}) \mathrm{ppm}$; MS m/z (I $\left.\mathrm{I}_{\mathrm{rel}}, \%\right): 523\left[\mathrm{M}^{+}\right](5), 458\left[\mathrm{M}-\mathrm{F}-\mathrm{NO}_{2}\right]^{+}(2), 378\left[\mathrm{M}-\mathrm{Ph}-\mathrm{CF}_{3}\right]^{+}(12), 287$ (100), $145\left[\mathrm{Ph}-\mathrm{CF}_{3}\right]^{+}(90)$; HRMS (ESI ${ }^{+}$) $\mathrm{m} / z$ calcd for $\mathrm{C}_{19} \mathrm{H}_{11} \mathrm{~N}_{3} \mathrm{O}_{3} \mathrm{Cl}_{3} \mathrm{SF}_{3} \mathrm{Na}\left[\mathrm{M}+\mathrm{Na}^{+}\right.$: 545.9436; found: 545.9433 .

1-Methyl-4-nitro-3-(morpholin-4-yl)-1H-pyrazole-5-carbaldehyde (22) was prepared according to the literature [26] in $48 \%$ yield.

Synthesis of 5-\{[3-(Morpholin-4-yl)-4-nitro-1H-pyrazol-5-yl]methylidene\}-3-[4-(propan-2-yl)phenyl]-2(2,3,3-trichloro-1-nitropropylidene)-1,3-thiazolidin-4-one (23a) (General method). To a suspension of thiazolidinone 19a $(0.408 \mathrm{~g}, 1.0 \mathrm{mmol})$ in acetic acid $(10 \mathrm{~mL})$, a solution of 1-methyl-3-(morpholin-4-yl)-4-nitro- $1 H$-pyrazole-5-carbaldehyde $(0.240 \mathrm{~g}, 1.0 \mathrm{mmol})$ in acetic acid $(5 \mathrm{~mL})$ and triethylamine $(0.152 \mathrm{~g}, 1.5 \mathrm{mmol})$ was added. Subsequently, the mixture was refluxed for $4 \mathrm{~h}$. After cooling, the solvent was removed and $\mathrm{MeOH}(5 \mathrm{~mL})$ added. The resulting precipitate was filtered off and washed with cold $\mathrm{MeOH}(2 \times 5 \mathrm{~mL})$. The product was dried in vacuo. Yield $0.592 \mathrm{~g}$ (94\%), orange solid, mixture of two isomers (100: 16), m.p. $212-213{ }^{\circ} \mathrm{C}$. IR (ATR) $v_{\max }=2961,1735$, $1535,1290,1178,683 \mathrm{~cm}^{-1} .{ }^{1} \mathrm{H}$ NMR major isomer $\left(400 \mathrm{MHz}, \mathrm{DMSO}-\mathrm{d}_{6}\right) \delta=1.21(6 \mathrm{H}, \mathrm{d}, J=6.9 \mathrm{~Hz}$, $\left.\mathrm{CH}_{3} i-\mathrm{Pr}\right), 2.99(1 \mathrm{H}, \mathrm{sep}, J=6.8 \mathrm{~Hz}, \mathrm{CH} i-\mathrm{Pr}), 3.22-3.28\left(4 \mathrm{H}, \mathrm{m}, \mathrm{NCH}_{2}\right), 3.72-3.78\left(4 \mathrm{H}, \mathrm{m}, \mathrm{OCH}_{2}\right), 3.85$ $\left(3 \mathrm{H}, \mathrm{s}, \mathrm{NCH}_{3}\right), 7.33-7.53(4 \mathrm{H}, \mathrm{m}, \mathrm{H} \mathrm{Ar}), 7.90(1 \mathrm{H}, \mathrm{s},=\mathrm{CH}) \mathrm{ppm} ;{ }^{13} \mathrm{C}$ NMR major isomer $(100 \mathrm{MHz}$, DMSO-d $\left._{6}\right) \delta=23.5,23.8\left(\mathrm{CH}_{3} i-\mathrm{Pr}\right), 33.6(\mathrm{CH} i-\mathrm{Pr}), 39.0\left(\mathrm{NCH}_{3}\right), 49.9\left(\mathrm{NCH}_{2}\right), 65.9\left(\mathrm{OCH}_{2}\right), 120.1$, $121.4(\mathrm{CH}), 121.8,123.5\left(\mathrm{CNO}_{2}\right), 127.0(\mathrm{CH}), 127.4(2 \times \mathrm{CH}), 128.9(\mathrm{CH}), 129.5,130.9,131.7,137.4,151.1$

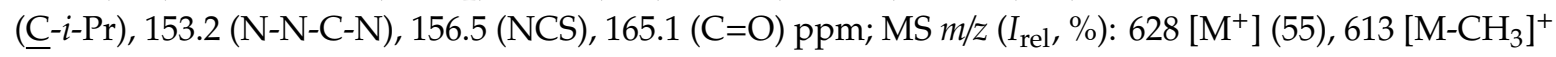


(83), $598\left[\mathrm{M}-2\left(\mathrm{CH}_{3}\right)\right]^{+}(22), 582\left[\mathrm{M}-\mathrm{NO}_{2}\right]^{+}(20), 499\left[\mathrm{M}-\mathrm{C}_{2} \mathrm{Cl}_{3}\right]^{+}$(10), 146 (100); HRMS (ESI $\left.{ }^{+}\right) \mathrm{m} / \mathrm{z}$ calcd for $\mathrm{C}_{24} \mathrm{H}_{23} \mathrm{~N}_{6} \mathrm{O}_{6} \mathrm{Cl}_{3} \mathrm{SNa}$ [M + Na] $]^{+}$: 651.0363; found: 651.0360 .

5-\{[3-(Morpholin-4-yl)-4-nitro-1H-pyrazol-5-yl]methylidene\}-2- $\quad$ (2,3,3-trichloro-1-nitropropylidene) -3-[4-(trifluoromethyl)phenyl]-1,3-thiazolidin-4-one (23b). Same procedure as for $\mathbf{2 3 a}$, using thiazolidinone $19 b$ (0.434 g, $1.0 \mathrm{mmol}), 1$-methyl-3-(morpholin-4-yl)-4-nitro-1H-pyrazole-5-carbaldehyde (0.240 g, 1.0 $\mathrm{mmol})$ and triethylamine $(0.152 \mathrm{~g}, 1.5 \mathrm{mmol})$. The mixture was refluxed for $2 \mathrm{~h}$. Yield $0.636 \mathrm{~g}(97 \%)$, mixture of two isomers (100: 17), orange solid, m.p. $226-227^{\circ} \mathrm{C}$. IR (ATR) $v_{\max }=1733,1538,1291,1171$, $1065,630 \mathrm{~cm}^{-1} .{ }^{1} \mathrm{H}$ NMR major isomer $\left(400 \mathrm{MHz}, \mathrm{DMSO}_{6}\right) \delta=3.22-3.29\left(4 \mathrm{H}, \mathrm{m}, \mathrm{NCH}_{2}\right), 3.72-3.79$ $\left(4 \mathrm{H}, \mathrm{m}, \mathrm{OCH}_{2}\right), 3.85\left(3 \mathrm{H}, \mathrm{s}, \mathrm{NCH}_{3}\right), 7.81-8.04(4 \mathrm{H}, \mathrm{m}, 4 \mathrm{H} \mathrm{Ar}), 7.93(1 \mathrm{H}, \mathrm{s},=\mathrm{CH}) \mathrm{ppm} ;{ }^{13} \mathrm{C} \mathrm{NMR} \mathrm{major}^{2}$ isomer $\left(100 \mathrm{MHz}, \mathrm{DMSO}-\mathrm{d}_{6}\right) \delta=39.1\left(\mathrm{NCH}_{3}\right), 49.9\left(\mathrm{NCH}_{2}\right), 65.9\left(\mathrm{OCH}_{2}\right), 120.1,121.6(\mathrm{pyr}-\mathrm{CH}), 121.7$ $(\mathrm{CH}), 123.9\left(\mathrm{~J}_{\mathrm{C}, \mathrm{F}}=272.7 \mathrm{~Hz}, \mathrm{CF}_{3}\right), 123.6\left(\mathrm{CNO}_{2}\right), 126.3\left(\mathrm{~J}_{\mathrm{C}, \mathrm{F}}=3.9 \mathrm{~Hz}, \mathrm{CH}\right), 126.8\left(\mathrm{~J}_{\mathrm{C}, \mathrm{F}}=3.9 \mathrm{~Hz}, \mathrm{CH}\right)$, $129.0(\mathrm{CH}), 130.2,130.3(\mathrm{CH}), 130.8,131.2\left(\mathrm{~J}_{\mathrm{C}, \mathrm{F}}=32.1 \mathrm{~Hz}, \mathrm{CCF}_{3}\right), 137.3,137.7,153.2,156.2(\mathrm{NCS}), 164.9$ $(\mathrm{C}=\mathrm{O}) \mathrm{ppm} ; \mathrm{MS} m / z\left(\mathrm{I}_{\mathrm{rel}}, \%\right): 654\left[\mathrm{M}^{+}\right](35), 639\left[\mathrm{M}-\mathrm{CH}_{3}\right]^{+}(60), 619[\mathrm{M}-\mathrm{Cl}]^{+}(8), 145\left[\mathrm{Ph}_{-} \mathrm{CF}_{3}\right]^{+}(50)$, 127 (100); $\mathrm{HRMS}\left(\mathrm{ESI}^{+}\right.$) $\mathrm{m} / \mathrm{z}$ calcd for $\mathrm{C}_{22} \mathrm{H}_{16} \mathrm{~N}_{6} \mathrm{O}_{6} \mathrm{Cl}_{3} \mathrm{SF}_{3} \mathrm{Na}[\mathrm{M}+\mathrm{Na}]^{+}$: 676.9767; found: 676.9769 .

1,1'-(3,4,4-Trichloro-2-nitrobuta-1,3-diene-1,1-diyl)bis(1H-benzotriazole) (24) was prepared according to the literature [28] in $76 \%$ yield.

Synthesis of N-[1-(1H-Benzotriazol-1-yl)-3,4,4-trichloro-2-nitrobuta-1,3-dien-1-yl]-2-methoxyaniline (25a) (General method). To a suspension of azole $24(0.437 \mathrm{~g}, 1.0 \mathrm{mmol})$ in $\mathrm{MeOH}(10 \mathrm{~mL})$ at $0{ }^{\circ} \mathrm{C}$, 2-methoxyaniline $(0.129 \mathrm{~g}, 1.05 \mathrm{mmol})$ was slowly added. The mixture was allowed to reach r.t. and stirred for another $3 \mathrm{~h}$. After evaporation of the solvent, $\mathrm{HCl}(10 \%, 10 \mathrm{~mL})$ was added and the resulting sludge was stirred for $20 \mathrm{~min}$. The precipitate was then filtered off and washed with $\mathrm{HCl}$ $(10 \%, 5 \mathrm{~mL})$, cold water $(5 \mathrm{~mL})$ and cold $\mathrm{Et}_{2} \mathrm{O}(2 \times 5 \mathrm{~mL})$. The product was dried in vacuo. Yield 0.392 $\mathrm{g}(89 \%)$, yellow solid, m.p. $128-129^{\circ} \mathrm{C}$. IR (KBr) $v_{\max }=1621,1580,1463,1252,1023,753 \mathrm{~cm}^{-1} .{ }^{1} \mathrm{H}$ $\operatorname{NMR}\left(200 \mathrm{MHz}, \mathrm{CDCl}_{3}\right) \delta=3.91\left(3 \mathrm{H}, \mathrm{s}, \mathrm{OCH}_{3}\right), 6.15(1 \mathrm{H}, \mathrm{dd}, J=1.3 \mathrm{~Hz}, J=8.0 \mathrm{~Hz}, \mathrm{H} \mathrm{Ar}), 6.46(1 \mathrm{H}$, ddd $J=7.7 \mathrm{~Hz}, J=1.2 \mathrm{~Hz}, J=7.5 \mathrm{~Hz}, \mathrm{H} \mathrm{Ar}), 6.82(1 \mathrm{H}, \mathrm{dd}, J=1.5 \mathrm{~Hz}, J=8.3 \mathrm{~Hz}, \mathrm{H} \mathrm{Ar}), 7.00(1 \mathrm{H}$, $\mathrm{ddd}, J=8.3 \mathrm{~Hz}, J=1.4 \mathrm{~Hz}, J=7.5 \mathrm{~Hz}, \mathrm{H} \mathrm{Ar}), 7.27-7.42(3 \mathrm{H}, \mathrm{m}, \mathrm{H}$ Bzt), 8.02-8.06 (1H, m, H Bzt), 11.65 $(1 \mathrm{H}, \mathrm{s}, \mathrm{NH}) \mathrm{ppm} ;{ }^{13} \mathrm{C} \mathrm{NMR}\left(50 \mathrm{MHz}, \mathrm{CDCl}_{3}\right) \delta=55.9\left(\mathrm{OCH}_{3}\right), 109.8(\mathrm{CH} \mathrm{Bzt}), 111.3(\mathrm{CH} \mathrm{Ar}), 120.6$ (CH Ar), 120.9 (CH Bzt), 122.2 (CH Ar), $123.8(\mathrm{CNH}), 125.3$ (CH Bzt), 128.6 (CH Ar), 129.5 (CH Bzt), $131.9,145.3(\mathrm{NCN}), 145.9,151.4\left(\mathrm{COCH}_{3}\right) \mathrm{ppm}$, three $\mathrm{C}$ signals from butadiene-chain due to their low intensity could not be detected; $\mathrm{MS} \mathrm{m} / \mathrm{z}\left(\mathrm{I}_{\mathrm{rel}}, \%\right): 439\left[\mathrm{M}^{+}\right](4), 403[\mathrm{M}-\mathrm{HCl}]^{+}(3), 358\left[\mathrm{M}-\mathrm{Cl}_{-} \mathrm{NO}_{2}\right]^{+}(3)$, $331\left[\mathrm{M}-\mathrm{Ph}-\mathrm{OCH}_{3}\right]^{+}(6), 122\left[\mathrm{H}_{2} \mathrm{NPhOCH}_{3}\right]^{+}(100)$; HRMS $\left(\mathrm{ESI}^{+}\right) \mathrm{m} / z$ calcd for $\mathrm{C}_{17} \mathrm{H}_{13} \mathrm{~N}_{5} \mathrm{O}_{3} \mathrm{Cl}_{3}[\mathrm{M}+$ $\mathrm{H}]^{+}$: 440.0079; found: 440.0077 .

N-[1-(1H-Benzotriazol-1-yl)-3,4,4-trichloro-2-nitrobuta-1,3-dien-1-yl]-2-ethoxyaniline (25b). Same procedure as for 25a, using 2-ethoxyaniline ( $0.144 \mathrm{~g}, 1.05 \mathrm{mmol})$. Yield $0.355 \mathrm{~g}(78 \%)$, orange solid, m.p. 145-146 ${ }^{\circ} \mathrm{C}$. IR (ATR) $v_{\max }=1620,1583,1471,1241,1147,741 \mathrm{~cm}^{-1} .{ }^{1} \mathrm{H}$ NMR $\left(400 \mathrm{MHz}, \mathrm{CDCl}_{3}\right) \delta=1.56(3 \mathrm{H}, \mathrm{t}$, $\left.J=7.0 \mathrm{~Hz}, \mathrm{OCH}_{2} \mathrm{CH}_{3}\right), 4.15\left(2 \mathrm{H}, \mathrm{q}, J=6.7 \mathrm{~Hz}, \mathrm{OCCH}_{2} \mathrm{CH}_{3}\right), 5.99(1 \mathrm{H}, \mathrm{dd}, J=1.0 \mathrm{~Hz}, J=8.0 \mathrm{~Hz}, \mathrm{H} \mathrm{Ar})$, $6.41(1 \mathrm{H}, \mathrm{ddd}, J=7.6 \mathrm{~Hz}, J=1.0 \mathrm{~Hz}, J=7.9 \mathrm{~Hz}, \mathrm{H} \mathrm{Ar}), 6.82(1 \mathrm{H}, \mathrm{dd}, J=8.3 \mathrm{~Hz}, J=0.9 \mathrm{~Hz}, \mathrm{H} \mathrm{Ar}), 6.97$ $(1 \mathrm{H}, \mathrm{ddd}, J=8.0 \mathrm{~Hz}, J=1.2 \mathrm{~Hz}, J=7.8 \mathrm{~Hz}, \mathrm{H} \mathrm{Ar}), 7.31(1 \mathrm{H}, \mathrm{d}, J=8.0 \mathrm{~Hz}, \mathrm{H} \mathrm{Bzt}), 7.37(\mathrm{ddd}, J=7.0 \mathrm{~Hz}, J$ $=1.1 \mathrm{~Hz}, J=8.1 \mathrm{~Hz}, \mathrm{H} \mathrm{Bzt}), 7.43(1 \mathrm{H}, \mathrm{t}, J=7.36 \mathrm{~Hz}, \mathrm{H} \mathrm{Bzt}), 8.06(1 \mathrm{H}, \mathrm{d}, J=8.2 \mathrm{~Hz}, \mathrm{H} \mathrm{Bzt}) \mathrm{ppm} ;{ }^{13} \mathrm{C}$ NMR $\left(100 \mathrm{MHz}, \mathrm{CDCl}_{3}\right) \delta=14.7\left(\mathrm{CH}_{3}\right), 64.7\left(\mathrm{OCH}_{2}\right), 109.8(\mathrm{CH}), 112.2(\mathrm{CH}), 120.6(\mathrm{CH}), 120.7(\mathrm{CH})$, $121.5(\mathrm{CH}), 124.0,125.3(\mathrm{CH}), 128.3(\mathrm{CH}), 129.5(\mathrm{CH}), 131.9,145.3,145.6,150.6 \mathrm{ppm}$, three $\mathrm{C}$ signals from butadiene-chain due to their low intensity could not be detected; MS $\mathrm{m} / z\left(I_{\text {rel }}, \%\right): 453\left[\mathrm{M}^{+}\right](1)$, $417\left[\mathrm{M}-\mathrm{HCl}^{+}(1), 390\left[\mathrm{M}-\mathrm{Cl}-\mathrm{N}_{2}\right]^{+}\right.$(1), $135\left[\mathrm{NPhOEt}_{2}\right]^{+}$(38), 100 (100); HRMS $\left(\mathrm{ESI}^{+}\right) \mathrm{m} / \mathrm{z}$ calcd for $\mathrm{C}_{18} \mathrm{H}_{15} \mathrm{~N}_{5} \mathrm{O}_{3} \mathrm{Cl}_{3}[\mathrm{M}+\mathrm{H}]^{+}$: 454.0235; found: 454.0234 .

N'-[1-(1H-Benzotriazol-1-yl)-3,4,4-trichloro-2-nitrobuta-1,3-dien-1-yl]-N,N-dimethylbenzene-1,4-di-amine (25c). Same procedure as for 25a, using $\mathrm{N}, \mathrm{N}$-dimethylbenzene-1,4-diamine $(0.143 \mathrm{~g}, 1.05 \mathrm{mmol})$ keeping the reaction mixture at $0{ }^{\circ} \mathrm{C}$ until completion. Yield $0.413 \mathrm{~g}(91 \%)$, dark red solid, m.p. $147-148{ }^{\circ} \mathrm{C}$. IR (ATR) $v_{\max }=2891,1620,1491,1358,1168,818 \mathrm{~cm}^{-1} .{ }^{1} \mathrm{H} \mathrm{NMR}\left(400 \mathrm{MHz}, \mathrm{CDCl}_{3}\right) \delta=2.81(6 \mathrm{H}, \mathrm{s}$, 
$\left.\mathrm{NCH}_{3}\right), 6.31(2 \mathrm{H}, \mathrm{d}, J=9.0 \mathrm{~Hz}, \mathrm{H} \mathrm{Ar}), 6.60(2 \mathrm{H}, \mathrm{d}, J=9.0 \mathrm{~Hz}, \mathrm{H} \mathrm{Ar}), 7.35-7.44(3 \mathrm{H}, \mathrm{m}, \mathrm{H} \mathrm{Bzt}), 8.04(1 \mathrm{H}$, $\mathrm{d}, J=8.4 \mathrm{~Hz}, \mathrm{H}$ Bzt $), 11.88(1 \mathrm{H}, \mathrm{s}, \mathrm{NH}) \mathrm{ppm} ;{ }^{13} \mathrm{C} \mathrm{NMR}\left(100 \mathrm{MHz}, \mathrm{CDCl}_{3}\right) \delta=40.1\left(\mathrm{CH}_{3}\right), 109.8(\mathrm{CH})$, 112.2 (CH), 117.0, $120.6(\mathrm{CH}), 120.9,123.8(\mathrm{CH}), 125.2(\mathrm{CH}), 128.0,129.3(\mathrm{CH}), 129.5,145.3,146.8,149.4$ $\mathrm{ppm}$, three $\mathrm{C}$ signals from butadiene-chain due to their low intensity could not be detected; MS $\mathrm{m} / \mathrm{z}$ $\left.\left(I_{\text {rel }}, \%\right): 452\left[\mathrm{M}^{+}\right](2), 416[\mathrm{M}-\mathrm{Cl}]^{+}(1), 388\left[\mathrm{M}-\mathrm{HCl}-\mathrm{N}_{2}\right]^{+}(1), 119[\mathrm{Bzt}+\mathrm{H}]^{+}(100) ; \mathrm{HRMS}_{(\mathrm{ESI}}{ }^{+}\right) \mathrm{m} / \mathrm{z}$ calcd for $\mathrm{C}_{18} \mathrm{H}_{16} \mathrm{~N}_{6} \mathrm{O}_{2} \mathrm{Cl}_{3}[\mathrm{M}+\mathrm{H}]^{+}$: 453.0395; found: 453.0395 .

1-[3,4,4-Trichloro-1-(2,3-dihydro-1H-indol-1-yl)-2-nitrobuta-1,3-dien-1-yl]-1H-benzotriazole (25f). Same procedure as for $25 \mathrm{a}$, using $1 H$-indoline $(0.125 \mathrm{~g}, 1.05 \mathrm{mmol})$. Yield $0.354 \mathrm{~g}(87 \%)$, yellow solid, m.p. 178-179 ${ }^{\circ} \mathrm{C}$. IR (ATR) $v_{\max }=3065,1533,1295,1023,905,655 \mathrm{~cm}^{-1} .{ }^{1} \mathrm{H}$ NMR $\left(400 \mathrm{MHz}, \mathrm{CDCl}_{3}\right) \delta=$ 3.34-3.46 (2H, m, $\mathrm{CH}_{2}$ ind), 3.89-4.25 (2H, $\mathrm{m}, \mathrm{CH}_{2}$ ind), 5.06-6.38 (1H, s, $\mathrm{H}$ ind $), 6.66-7.00(1 \mathrm{H}, \mathrm{m}, \mathrm{H}$ Bzt), $7.06(1 \mathrm{H}, \mathrm{s}, \mathrm{H}$ ind $), 7.29-7.36(1 \mathrm{H}, \mathrm{m}, \mathrm{H}$ ind $), 7.40-7.88$ (3H, $\mathrm{m}, 2 \mathrm{H}$ Bzt, $1 \mathrm{H}$ ind $), 8.16(1 \mathrm{H}, \mathrm{d}, J$ $=8.1 \mathrm{~Hz}, \mathrm{H}$ Bzt $)$ ppm; ${ }^{13} \mathrm{C} \mathrm{NMR}\left(100 \mathrm{MHz}, \mathrm{CDCl}_{3}\right) \delta=29.2\left(\mathrm{CH}_{2}\right), 54.5\left(\mathrm{CH}_{2}\right), 113.9\left(\mathrm{CNO}_{2}\right), 121.1$ $(2 \times \mathrm{CH}), 123.2,125.9(2 \times \mathrm{CH}), 126.0(\mathrm{CH}), 127.9(\mathrm{CH}), 128.0(\mathrm{CH}), 130.0(\mathrm{CH}), 131.8\left(\mathrm{CCl}_{2}\right), 141.2$ $(\mathrm{NCN}), 146.1,146.2 \mathrm{ppm} ; \mathrm{MS} \mathrm{m} / \mathrm{z}\left(\mathrm{I}_{\mathrm{rel}}, \%\right): 435\left[\mathrm{M}^{+}\right](12), 289$ [M-indolin- $\left._{2}\right]^{+}(34), 271$ [M-Bzt- $\left.^{+} \mathrm{NO}_{2}\right]^{+}$ (79), 118 [benzotriazole] ${ }^{+}(100)$; HRMS $\left(\mathrm{ESI}^{+}\right) \mathrm{m} / \mathrm{z}$ calcd for $\mathrm{C}_{18} \mathrm{H}_{13} \mathrm{~N}_{5} \mathrm{O}_{2} \mathrm{Cl}_{3}[\mathrm{M}+\mathrm{H}]^{+}: 436.0129$; found: 436.0127.

Synthesis of 5-Methoxy-8-(2,3,3-trichloro-1-nitroprop-2-en-1-ylidene)-7-azabicyclo[4.2.0]octa-1,3,5- triene (26a) (General method). A suspension of azole $25 \mathrm{a}(0.441 \mathrm{~g}, 1.0 \mathrm{mmol})$ in $\mathrm{MeOH}(10 \mathrm{~mL})$ was stirred at reflux for $5 \mathrm{~h}$. Subsequently, the mixture was concentrated and cooled to $0{ }^{\circ} \mathrm{C}$. The precipitate was filtered off and washed with diethyl ether $(2 \times 5 \mathrm{~mL})$. Yield $0.119 \mathrm{~g}(37 \%)$, yellow solid, m.p. $168-169^{\circ} \mathrm{C}$. IR $(\mathrm{KBr})$ $v_{\max }=3112,1637,1536,1395,1083,859 \mathrm{~cm}^{-1} .{ }^{1} \mathrm{H} \mathrm{NMR}\left(200 \mathrm{MHz}, \mathrm{DMSO}-\mathrm{d}_{6}\right) \delta=3.96\left(3 \mathrm{H}, \mathrm{s}, \mathrm{OCH}_{3}\right)$, $7.30-7.42(2 \mathrm{H}, \mathrm{m}, \mathrm{CH}), 7.74(1 \mathrm{H}, \mathrm{dd}, J=2.3 \mathrm{~Hz}, J=7.6 \mathrm{~Hz}, \mathrm{CH}), 12.40(1 \mathrm{H}, \mathrm{s}, \mathrm{NH}) \mathrm{ppm} ;{ }^{13} \mathrm{C}$ NMR $(50$ MHz, DMSO-d 6 ) $\delta=57.0\left(\mathrm{OCH}_{3}\right), 110.9(\mathrm{CH}), 114.3(\mathrm{CH}), 119.5,123.9(\mathrm{CH}), 124.0,125.9,130.6,134.0$,

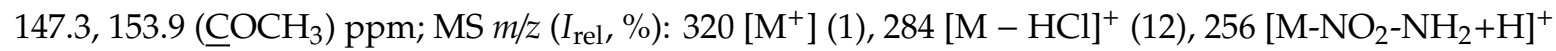
(40), 64 (100); $\mathrm{HRMS}\left(\mathrm{ESI}^{+}\right.$) $\mathrm{m} / \mathrm{z}$ calcd for $\mathrm{C}_{11} \mathrm{H}_{8} \mathrm{~N}_{2} \mathrm{O}_{3} \mathrm{Cl}_{3}[\mathrm{M}+\mathrm{H}]^{+}: 320.9595$; found: 320.9594 .

5-Ethoxy-8-(2,3,3-trichloro-1-nitroprop-2-en-1-ylidene)-7-azabicyclo[4.2.0]octa-1,3,5-triene (26b). Same procedure as for 26a, using azole 25b $(0.455,1.0 \mathrm{mmol})$ and refluxing in EtOH for $10 \mathrm{~h}$. Yield $0.091 \mathrm{~g}(27 \%)$, yellow solid, m.p. 204-205 ${ }^{\circ} \mathrm{C}$. IR (KBr) $v_{\max }=2992,1647,1533,1378,1234,554 \mathrm{~cm}^{-1}$. ${ }^{1} \mathrm{H}$ NMR $\left(400 \mathrm{MHz}, \mathrm{DMSO}_{6}\right) \delta=1.42\left(3 \mathrm{H}, \mathrm{t}, J=7.0 \mathrm{~Hz}, \mathrm{OCH}_{2} \mathrm{CH}_{3}\right), 4.16-4.26\left(2 \mathrm{H}, \mathrm{m}, \mathrm{OCH}_{2} \mathrm{CH}_{3}\right)$, 7.30-7.38 (2H, m, CH), $7.71(1 \mathrm{H}, \mathrm{dd}, J=1.5 \mathrm{~Hz}, J=8.2 \mathrm{~Hz}, \mathrm{CH}), 12.37(1 \mathrm{H}, \mathrm{s}, \mathrm{NH}) \mathrm{ppm} ;{ }^{13} \mathrm{C}$ NMR $(100$ MHz, DMSO-d $\left.{ }_{6}\right) \delta=14.5\left(\mathrm{CH}_{3}\right), 65.4\left(\mathrm{OCH}_{2}\right), 110.7(\mathrm{CH}), 114.9(\mathrm{CH}), 119.5,123.9(\mathrm{CH}), 124.0,125.9$, 130.6, 133.9, 146.5, 153.9 ppm; MS m/z (I rel $\left._{1} \%\right): 334\left[\mathrm{M}^{+}\right]$(3), $299[\mathrm{M}-\mathrm{Cl}]^{+}(52), 271$ [M-Cl-EtO+H] ${ }^{+}$ (100); HRMS $\left(\right.$ ESI $\left.^{+}\right) \mathrm{m} / z$ calcd for $\mathrm{C}_{12} \mathrm{H}_{10} \mathrm{~N}_{2} \mathrm{O}_{3} \mathrm{Cl}_{3}[\mathrm{M}+\mathrm{H}]^{+}$: 334.9752; found: 334.9750.

Synthesis of N,N-Dimethyl-8-(2,3,3-trichloro-1-nitroprop-2-en-1-ylidene)-7-azabicyclo[4.2.0]octa-1,3,5trien-3-amine (26c). A suspension of azole $25 \mathrm{c}(0.454 \mathrm{~g}, 1.0 \mathrm{mmol})$ in THF $(20 \mathrm{~mL})$ was stirred at reflux for $10 \mathrm{~h}$. After evaporation of the solvent, dilute $\mathrm{HCl}(10 \mathrm{~mL})$ was added and the mixture stirred for $15 \mathrm{~min}$. Subsequently, the mixture was extracted with chloroform and purified by column chromatography using petroleum ether - ethyl acetate (1:1). Yield $0.084 \mathrm{~g}(25 \%)$, red solid, m.p. 171-173 ${ }^{\circ} \mathrm{C}$. IR (KBr) $v_{\max }=2856,1567,1369,1063,846,550 \mathrm{~cm}^{-1} .{ }^{1} \mathrm{H}$ NMR $\left(400 \mathrm{MHz}, \mathrm{DMSO}-\mathrm{d}_{6}\right) \delta=2.97(6 \mathrm{H}$, $\left.\mathrm{s}, \mathrm{N}\left(\mathrm{CH}_{3}\right)_{2}\right), 7.22(1 \mathrm{H}, \mathrm{d}, J=2.0 \mathrm{~Hz}, \mathrm{CH}), 7.32(1 \mathrm{H}, \mathrm{d}, J=9.0 \mathrm{~Hz}, \mathrm{CH}), 7.36(1 \mathrm{H}, \mathrm{dd}, J=2.1 \mathrm{~Hz}, J=9.2$ $\mathrm{Hz}, \mathrm{CH}), 12.65(1 \mathrm{H}, \mathrm{s}, \mathrm{NH}) \mathrm{ppm} ;{ }^{13} \mathrm{C}$ NMR $\left(100 \mathrm{MHz}, \mathrm{DMSO}-\mathrm{d}_{6}\right) \delta=40.3(\mathrm{Me}), 98.6(\mathrm{CH}), 117.8(\mathrm{CH})$, $119.9,121.1(\mathrm{CH}), 124.0,125.6,130.9,133.5,147.5\left(\mathrm{CNMe}_{2}\right), 153.1 \mathrm{ppm} ; \mathrm{MS} \mathrm{m} / z\left(I_{\text {rel }}, \%\right): 333\left[\mathrm{M}^{+}\right](27)$, $298[\mathrm{M}-\mathrm{Cl}]^{+}(40), 270\left[\mathrm{M}-\mathrm{NO}_{2}-\mathrm{NH}_{3}\right]^{+}(100) ; \mathrm{HRMS}\left(\mathrm{ESI}^{+}\right) \mathrm{m} / z$ calcd for $\mathrm{C}_{12} \mathrm{H}_{11} \mathrm{~N}_{3} \mathrm{O}_{2} \mathrm{Cl}_{3}[\mathrm{M}+\mathrm{H}]^{+}$: 333.9917; found: 333.9919.

Synthesis of N'-[6-(Dichloromethyl)-2-methyl-5-nitropyrimidin-4-yl]-N, N-dimethylbenzene-1,4- diamine (27c). To a solution of the nitrobutadiene $25 \mathrm{c}(0.454 \mathrm{~g}, 1.0 \mathrm{mmol})$ and acetamidine hydrochloride $(0.284 \mathrm{~g}, 3.0$ mmol $)$ in $20 \mathrm{~mL}$ dry THF, sodium hydride $(0.160 \mathrm{~g}, 4.0 \mathrm{mmol}, 60 \%)$ was added at $0{ }^{\circ} \mathrm{C}$. The solution was thoroughly stirred for $1 \mathrm{~h}$ at $0{ }^{\circ} \mathrm{C}$ and then at r.t. for $2 \mathrm{~d}$. After evaporation of the solvent and 
addition of $10 \% \mathrm{HCl}(5 \mathrm{~mL})$ the precipitate was filtered off and washed with $10 \% \mathrm{HCl}(2 \times 10 \mathrm{~mL})$, cold water $(10 \mathrm{~mL})$, cold $\mathrm{MeOH}(2 \times 5 \mathrm{~mL})$ and dried in vacuo. Yield $0.232 \mathrm{~g}(65 \%)$, yellow solid, m.p. 124-125 ${ }^{\circ} \mathrm{C}$. IR $(\mathrm{KBr}) v_{\max }=3321,1612,1573\left(\mathrm{NO}_{2}\right), 1517,1204,746 \mathrm{~cm}^{-1} .{ }^{1} \mathrm{H}$ NMR $\left(400 \mathrm{MHz}, \mathrm{CDCl}_{3}\right)$ $\delta=2.64\left(3 \mathrm{H}, \mathrm{s}, \mathrm{CCH}_{3}\right), 2.99\left(6 \mathrm{H}, \mathrm{s}, \mathrm{NCH}_{3}\right), 6.74(2 \mathrm{H}, \mathrm{d}, J=9.0 \mathrm{~Hz}, \mathrm{H} \mathrm{Ar}), 7.40(2 \mathrm{H}, \mathrm{d}, J=8.9 \mathrm{~Hz}, \mathrm{H} \mathrm{Ar})$, $7.42\left(1 \mathrm{H}, \mathrm{s}, \mathrm{CHCl}_{2}\right), 9.73(1 \mathrm{H}, \mathrm{s}, \mathrm{NH}) \mathrm{ppm} ;{ }^{13} \mathrm{C} \mathrm{NMR}\left(100 \mathrm{MHz}, \mathrm{CDCl}_{3}\right) \delta=26.5\left(\mathrm{CH}_{3}\right), 40.5\left(\mathrm{NCH}_{3}\right)$, $66.8\left(\mathrm{CHCl}_{2}\right), 112.3(\mathrm{CH}), 122.8,124.5(\mathrm{CH}), 125.3,148.9,153.2,160.6,171.0 \mathrm{ppm} ; \mathrm{MS} \mathrm{m} / z\left(I_{\text {rel }}, \%\right): 355$ $\left[\mathrm{M}^{+}\right]$(39), 136 [phenylendiamine+H] $]^{+}(100)$; HRMS $\left(\mathrm{ESI}^{+}\right) \mathrm{m} / z$ calcd for $\mathrm{C}_{14} \mathrm{H}_{16} \mathrm{~N}_{5} \mathrm{O}_{2} \mathrm{Cl}_{2}[\mathrm{M}+\mathrm{H}]^{+}$: 356.0676; found: 356.0674 .

6-(Dichloromethyl)-N,N,2-trimethyl-5-nitropyrimidin-4-amine (27d). Same procedure as for 27c, using diene $25 \mathrm{~d}(0.363 \mathrm{~g}, 1.0 \mathrm{mmol})$. The precipitate was further purified by column chromatography using

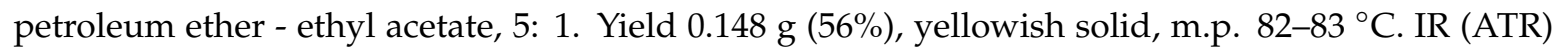
$v_{\max }=3048,1579\left(\mathrm{NO}_{2}\right), 1500,1338\left(\mathrm{NO}_{2}\right), 1195,746 \mathrm{~cm}^{-1} .{ }^{1} \mathrm{H} \mathrm{NMR}\left(400 \mathrm{MHz}, \mathrm{CDCl}_{3}\right) \delta=2.61$ $\left(3 \mathrm{H}, \mathrm{s}, \mathrm{CCH}_{3}\right), 3.09\left(6 \mathrm{H}, \mathrm{s}, \mathrm{NCH}_{3}\right), 6.98\left(1 \mathrm{H}, \mathrm{s}, \mathrm{CHCl}_{2}\right) ;{ }^{13} \mathrm{C} \mathrm{NMR}\left(100 \mathrm{MHz}, \mathrm{CDCl}_{3}\right) \delta=26.1\left(\mathrm{CH}_{3}\right)$, $39.3\left(\mathrm{NCH}_{3}\right), 65.4\left(\mathrm{CHCl}_{2}\right), 125.6\left(\mathrm{CNO}_{2}\right), 154.7,156.2,168.1 \mathrm{ppm} ; \mathrm{MS} \mathrm{m} / z\left(I_{\mathrm{rel}}, \%\right): 264\left[\mathrm{M}^{+}\right]$(55), 218 $\left[\mathrm{M}-\mathrm{NO}_{2}\right]^{+}(30), 182\left[\mathrm{M}-\mathrm{CHCl}_{2}+\mathrm{H}\right]^{+}(63), 135\left[\mathrm{M}-\mathrm{CHCl}_{2}-\mathrm{NO}_{2}\right]^{+}$(52). HRMS (ESI ${ }^{+}$) $\mathrm{m} / z$ calcd for $\mathrm{C}_{8} \mathrm{H}_{11} \mathrm{~N}_{4} \mathrm{O}_{2} \mathrm{Cl}_{2}[\mathrm{M}+\mathrm{H}]^{+}$: 265.0254; found: 265.0255 .

N-[6-(Dichloromethyl)-2-methyl-5-nitropyrimidin-4-yl]quinolin-8-amine (27e). Same procedure as for 27c, using azole $25 \mathrm{e}(0.462 \mathrm{~g}, 1.0 \mathrm{mmol})$ as starting material. Yield $0.178 \mathrm{~g}(49 \%)$, yellow solid, m.p. 217-219 ${ }^{\circ} \mathrm{C}$. IR $(\mathrm{KBr}) v_{\max }=3262,1573\left(\mathrm{NO}_{2}\right), 1298\left(\mathrm{NO}_{2}\right), 1208,766,590 \mathrm{~cm}^{-1} .{ }^{1} \mathrm{H} \mathrm{NMR}\left(400 \mathrm{MHz}, \mathrm{CDCl}_{3}\right) \delta=$ $2.82\left(3 \mathrm{H}, \mathrm{s}, \mathrm{CCH}_{3}\right), 7.37\left(1 \mathrm{H}, \mathrm{s}, \mathrm{CHCl}_{2}\right), 7.52(1 \mathrm{H}, \mathrm{dd}, J=8.3 \mathrm{~Hz}, J=4.2 \mathrm{~Hz}, \mathrm{H}$ quin), 7.58-7.63 $(2 \mathrm{H}, \mathrm{m}$, $\mathrm{H}$ quin), $8.21(1 \mathrm{H}, \mathrm{dd}, J=8.3 \mathrm{~Hz}, J=1.6 \mathrm{~Hz}, \mathrm{H}$ quin), $8.94(1 \mathrm{H}, \mathrm{dd}, J=4.2 \mathrm{~Hz}, J=1.6 \mathrm{~Hz}, \mathrm{H}$ quin), 9.04-9.07 (1H, m, H quin), $12.17(1 \mathrm{H}, \mathrm{s}, \mathrm{NH}) \mathrm{ppm} ;{ }^{13} \mathrm{C} \mathrm{NMR}\left(100 \mathrm{MHz}, \mathrm{CDCl}_{3}\right) \delta=26.6\left(\mathrm{CH}_{3}\right), 66.4$ $\left(\mathrm{CHCl}_{2}\right), 118.4(\mathrm{CH}), 122.0(\mathrm{CH}), 122.93(\mathrm{CH}), 124.6,127.0(\mathrm{CH}), 128.1,134.0,136.4(\mathrm{CH}), 139.3,149.0$ (CH), 152.1, 159.7, $170.9 \mathrm{ppm}$; MS m/z (I $\left.\mathrm{Iel}_{\text {rel }} \%\right): 363\left[\mathrm{M}^{+}\right](15), 317\left[\mathrm{M}-\mathrm{NO}_{2}\right]^{+}(100), 281\left[\mathrm{M}-\mathrm{CHCl}_{2}\right]^{+}$ (25), $235\left[\mathrm{M}\right.$ - quin] ${ }^{+}(12), 128$ [quinoline] $^{+}$(45); HRMS $\left(\mathrm{ESI}^{+}\right) \mathrm{m} / z$ calcd for $\mathrm{C}_{15} \mathrm{H}_{12} \mathrm{~N}_{5} \mathrm{O}_{2} \mathrm{Cl}_{2}[\mathrm{M}+\mathrm{H}]^{+}$: 364.0368; found: 364.0368 .

1-[6-(Dichloromethyl)-2-methyl-5-nitropyrimidin-4-yl]-2,3-dihydro-1H-indole (27f). Same procedure as for 27c, using diene $25 \mathrm{f}(0.437 \mathrm{~g}, 1.0 \mathrm{mmol})$, DMSO $(25 \mathrm{~mL})$ and $\mathrm{NaOH}(30 \%, 4 \mathrm{mmol})$. Yield $0.216 \mathrm{~g}(64 \%)$, yellow solid, m.p. $132-133^{\circ} \mathrm{C}$. IR (ATR) $v_{\max }=3048,1562\left(\mathrm{NO}_{2}\right), 1529,1340\left(\mathrm{NO}_{2}\right), 1209,768 \mathrm{~cm}^{-1} .{ }^{1} \mathrm{H}$ $\operatorname{NMR}\left(400 \mathrm{MHz}, \mathrm{CDCl}_{3}\right) \delta=2.72\left(3 \mathrm{H}, \mathrm{s}, \mathrm{CCH}_{3}\right), 3.20(2 \mathrm{H}, \mathrm{t}, J=7.9 \mathrm{~Hz}, \mathrm{H}$ ind $), 3.86(2 \mathrm{H}, \mathrm{t}, J=7.9 \mathrm{~Hz}, \mathrm{H}$ ind), $7.04\left(1 \mathrm{H}, \mathrm{s}, \mathrm{CHCl}_{2}\right), 7.11(1 \mathrm{H}, \mathrm{t}, J=7.4 \mathrm{~Hz}, \mathrm{H}$ ind $), 7.24-7.29(2 \mathrm{H}, \mathrm{m}, \mathrm{H}$ ind $), 7.93(1 \mathrm{H}, \mathrm{d}, J=8.1 \mathrm{~Hz}$, $\mathrm{H}$ ind) ppm; ${ }^{13} \mathrm{C} \mathrm{NMR}\left(100 \mathrm{MHz}, \mathrm{CDCl}_{3}\right) \delta=26.0(\mathrm{CH}), 28.8\left(\mathrm{CH}_{2}\right), 50.2\left(\mathrm{CH}_{2}\right), 65.3\left(\mathrm{CHCl}_{2}\right), 117.6$ $(\mathrm{CH}), 124.8(\mathrm{CH}), 125.1(\mathrm{CH}), 126.4,127.1(\mathrm{CH}), 132.3,142.1,151.6,156.8,168.7 \mathrm{ppm}$; MS m/z (I Irel $_{1} \%$ ): $338\left[\mathrm{M}^{+}\right](100), 323\left[{\left.\mathrm{M}-\mathrm{CH}_{3}\right]^{+}}^{+}(13), 257\left[\mathrm{M}-\mathrm{NO}_{2}-\mathrm{Cl}\right](48), 118\right.$ [indole $^{+}(24) ; \mathrm{HRMS}\left(\mathrm{ESI}^{+}\right) \mathrm{m} / \mathrm{z}$ calcd for $\mathrm{C}_{14} \mathrm{H}_{13} \mathrm{~N}_{4} \mathrm{O}_{2} \mathrm{Cl}_{2}[\mathrm{M}+\mathrm{H}]^{+}$: 339.0416; found: 339.0413 .

Synthesis of 1-[6-(Dichloromethyl)-2-methyl-5-nitropyrimidin-4-yl]-1H-indole (28). A solution of pyrimidine $27 \mathrm{f}(0.400 \mathrm{~g}, 1.2 \mathrm{mmol})$ and DDQ $(0.600 \mathrm{~g}, 2.7 \mathrm{mmol})$ in toluene $(10 \mathrm{~mL})$ was heated to reflux for 5 $\mathrm{h}$, allowed to cool down to r.t., and the precipitate filtered off. The filtrate was purified by column chromatography using petroleum ether-ethyl acetate, 25: 1 . The product was dried in vacuo. Yield $0.267 \mathrm{~g}(66 \%)$, m.p. $103-104{ }^{\circ} \mathrm{C}$. IR (ATR) $v_{\max }=3022,1589\left(\mathrm{NO}_{2}\right), 1342,1261\left(\mathrm{NO}_{2}\right), 1099,745 \mathrm{~cm}^{-1}$. ${ }^{1} \mathrm{H}$ NMR $\left(600 \mathrm{MHz}, \mathrm{CDCl}_{3}\right) \delta=2.92\left(3 \mathrm{H}, \mathrm{s}, \mathrm{CCH}_{3}\right), 6.79(1 \mathrm{H}, \mathrm{dd}, J=3.7 \mathrm{~Hz}, \mathrm{~J}=0.7 \mathrm{~Hz}, \mathrm{H}$ ind $), 7.01$ $\left(1 \mathrm{H}, \mathrm{s}, \mathrm{CHCl}_{2}\right), 7.12(1 \mathrm{H}, \mathrm{d}, J=3.7 \mathrm{~Hz}, \mathrm{H}$ ind $), 7.30(1 \mathrm{H}, \mathrm{t}, J=8.0 \mathrm{~Hz}, \mathrm{H}$ ind $), 7.36(1 \mathrm{H}, \mathrm{t}, J=8.4 \mathrm{~Hz}, \mathrm{H}$ ind), $7.63(1 \mathrm{H}, \mathrm{d}, J=8.2 \mathrm{~Hz}, \mathrm{H}$ ind $), 8.10(1 \mathrm{H}, \mathrm{d}, J=9.1 \mathrm{~Hz}, \mathrm{H}$ ind $) \mathrm{ppm} ;{ }^{13} \mathrm{C} \mathrm{NMR}\left(150 \mathrm{MHz}, \mathrm{CDCl}_{3}\right) \delta$ $=26.2\left(\mathrm{CH}_{3}\right), 64.8\left(\mathrm{CHCl}_{2}\right), 110.9(\mathrm{CH}), 114.1(\mathrm{CH}), 121.6(\mathrm{CH}), 123.8(\mathrm{CH}), 124.5(\mathrm{CH}), 124.9(\mathrm{CH})$, 128.3 130.4, 135.4, 150.5, 157.6, 170.5 ppm; MS m/z (I rel, \%): $336\left[\mathrm{M}^{+}\right](100), 291\left[\mathrm{M}+\mathrm{H}_{-N_{2}}\right]^{+}(42)$, $238\left[\mathrm{M}_{-} \mathrm{CH}_{3}-\mathrm{CHCl}_{2}\right]^{+}(48), 116$ [indole] $^{+}(62)$; $\mathrm{HRMS}\left(\mathrm{ESI}^{+}\right) \mathrm{m} / \mathrm{z}$ calcd for $\mathrm{C}_{14} \mathrm{H}_{11} \mathrm{~N}_{4} \mathrm{O}_{2} \mathrm{Cl}_{2}[\mathrm{M}+\mathrm{H}]^{+}$: 337.0259; found: 337.0261 . 
Synthesis of 1-[1-(1H-Benzotriazol-1-yl)-3,4,4-trichloro-2-nitrobuta-1,3-dien-1-yl]-4-(4-chlorophenyl)piperidin-4-ol (25g). Same procedure as for 25a, using 4-(4-chlorophenyl)piperidin-4-ol (0.223 g, 1.05 mmol). Yield $0.482 \mathrm{~g}(91 \%)$, yellowish solid, m.p. $149-150{ }^{\circ} \mathrm{C}$. IR (ATR) $v_{\max }=2931,1565,1498,1290$, 1005, $749 \mathrm{~cm}^{-1}$. ${ }^{1} \mathrm{H}$ NMR (400 MHz, $\left.\mathrm{CDCl}_{3}\right) \delta=1.83-2.03$ (2H, m, H pip), 2.33-2.61 (2H, m, H pip), 2.95-3.25 (2H, m, NCH 2$), 3.76-4.08\left(3 \mathrm{H}, \mathrm{m}, \mathrm{OH}+\mathrm{NCH}_{2}\right), 7.35-7.38(2 \mathrm{H}, \mathrm{m}, \mathrm{H}$ Ar), 7.45-7.47 (2H, m, H Ar), 7.47-7.79 (3H, m, H Bzt), 8.10-8.13 (1H, m, H Bzt) ppm; $\left.{ }^{13} \mathrm{C} \mathrm{NMR} \mathrm{(100} \mathrm{MHz,} \mathrm{CDCl}_{3}\right) \delta=38.3$ $\left(\mathrm{CH}_{2}\right), 47.3\left(\mathrm{CH}_{2}\right), 70.4(\mathrm{COH}), 110.4(\mathrm{CH}), 111.89\left(\mathrm{CNO}_{2}\right), 120.9(\mathrm{CH}), 124.8(\mathrm{CCl}), 125.9(2 \times \mathrm{CH})$, $126.0(\mathrm{CH}), 126.2\left(\mathrm{CCl}_{2}\right), 128.8(2 \times \mathrm{CH}), 130.6(\mathrm{CH}), 132.4,133.6(\mathrm{CCl}), 144.8,146.1,148.0(\mathrm{CNN}) \mathrm{ppm}$;

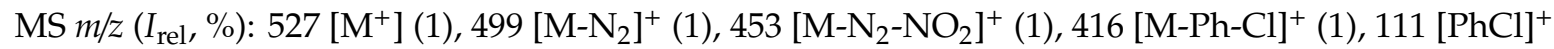
(20), 91 (100); HRMS (ESI ${ }^{+}$) $m / z$ calcd for $\mathrm{C}_{21} \mathrm{H}_{18} \mathrm{~N}_{5} \mathrm{O}_{3} \mathrm{Cl}_{4}[\mathrm{M}+\mathrm{H}]^{+}$: 538.0158; found: 528.0156.

\{4-[1-(1H-Benzotriazol-1-yl)-3,4,4-trichloro-2-nitrobuta-1,3-dien-1-yl]piperazin-1-yl\}(tetrahydrofuran-2yl)-methanone (25h). Same procedure as for 25a, using piperazin-1-yl(tetrahydrofuran-2-yl)methanone $(0.193 \mathrm{~g}, 1.05 \mathrm{mmol})$. Yield $0.426 \mathrm{~g}(85 \%)$, yellow solid, m.p. $170-172{ }^{\circ} \mathrm{C}$. IR $(\mathrm{KBr}) v_{\max }=2871,1657$, $1560,1290,1006,747 \mathrm{~cm}^{-1} .{ }^{1} \mathrm{H}$ NMR $\left(200 \mathrm{MHz}, \mathrm{CDCl}_{3}\right) \delta=1.80-2.11\left(3 \mathrm{H}, \mathrm{m}, \mathrm{CH}_{2}\right.$ fur $), 2.30-2.49(1 \mathrm{H}$, $\mathrm{m}, \mathrm{CH}_{2}$ fur $), 3.18-3.60\left(4 \mathrm{H}, \mathrm{m}, \mathrm{NCH}_{2}\right), 3.72-4.41\left(6 \mathrm{H}, \mathrm{m}, 2 \mathrm{NCH}_{2}, \mathrm{OCH}_{2}\right), 4.52-4.67(1 \mathrm{H}, \mathrm{m}, \mathrm{OCH})$, 7.48-7.80 (3H, m, H Bzt), 8.17-8.20 (1H, m, H Bzt) ppm; $\left.{ }^{13} \mathrm{C} \mathrm{NMR} \mathrm{(50} \mathrm{MHz,} \mathrm{CDCl}\right) \delta 25.7\left(\mathrm{CH}_{2}\right), 27.7$ $\left(\mathrm{CH}_{2}\right), 41.8\left(\mathrm{NCH}_{2}\right), 45.2\left(\mathrm{NCH}_{2}\right), 50.6\left(\mathrm{NCH}_{2}\right), 69.2\left(\mathrm{OCH}_{2}\right), 76.1(\mathrm{OCH}), 110.2(\mathrm{CH}), 116.2\left(\mathrm{CCl}_{2}\right)$, $120.6\left(\mathrm{CNO}_{2}\right), 121.1(\mathrm{CH}), 125.5,126.1(\mathrm{CH}), 129.9,130.7(\mathrm{CH}), 146.3,147.5(\mathrm{CNN}), 169.7(\mathrm{C}=\mathrm{O}) \mathrm{ppm}$; MS m/z (I $\left.I_{\text {rel }} \%\right): 500\left[\mathrm{M}^{+}\right](1), 465[\mathrm{M}-\mathrm{Cl}]^{+}(1), 426\left[\mathrm{M}-\mathrm{NO}_{2}-\mathrm{N}_{2}\right]^{+}(1), 337\left[\mathrm{M}-\mathrm{Bzt}-\mathrm{NO}_{2}+\mathrm{H}\right]^{+}(4), 119[\mathrm{Bzt}$ $+\mathrm{H}]^{+}(16), 92$ (100); HRMS $\left(\mathrm{ESI}^{+}\right) \mathrm{m} / \mathrm{z}$ calcd for $\mathrm{C}_{19} \mathrm{H}_{20} \mathrm{~N}_{6} \mathrm{O}_{4} \mathrm{Cl}_{3}[\mathrm{M}+\mathrm{H}]^{+}$: 501.0606; found: 501.0605 .

1-\{3,4,4-Trichloro-1-[4-(3-chlorophenyl)piperazin-1-yl]-2-nitrobuta-1,3-dien-1-yl\}-1H-benzotriazole (25i). Same procedure as for 25a, using 1-(3-methylphenyl)piperazine (0.207 g, $1.05 \mathrm{mmol})$. Yield $0.452 \mathrm{~g}$ (88\%), yellow solid, m.p. $68-70{ }^{\circ} \mathrm{C}$. IR $(\mathrm{KBr}) v_{\max }=2916,1594,1560,1291,942,768 \mathrm{~cm}^{-1} .{ }^{1} \mathrm{H}$ NMR $(200$ $\left.\mathrm{MHz}_{,} \mathrm{CDCl}_{3}\right) \delta=3.41-3.61\left(8 \mathrm{H}, \mathrm{m}, \mathrm{NCH}_{2}\right), 6.75-6.90(1 \mathrm{H}, \mathrm{m}, \mathrm{H} \mathrm{Ar}), 6.91-6.98$ (2H, m, H Ar), 7.18-7.24 $(1 \mathrm{H}, \mathrm{m}, \mathrm{H} \mathrm{Ar}), 7.40-7.68(3 \mathrm{H}, \mathrm{m}, \mathrm{H} \mathrm{Bzt}), 8.16(1 \mathrm{H}, \mathrm{d}, J=8.1 \mathrm{~Hz}, \mathrm{H} \mathrm{Bzt}) \mathrm{ppm} ;{ }^{13} \mathrm{C} \mathrm{NMR}\left(50 \mathrm{MHz}, \mathrm{CDCl}_{3}\right)$ $\delta=49.2\left(\mathrm{NCH}_{2}\right), 49.4\left(\mathrm{NCH}_{2}\right), 110.3(\mathrm{CH}), 115.1(\mathrm{CH}), 117.2(\mathrm{CH}), 121.1(\mathrm{CH}), 121.6(\mathrm{CH}), 124.3\left(\mathrm{CCl}_{2}\right)$, $125.3(\mathrm{CCl}), 126.1(\mathrm{CH}), 130.4(\mathrm{CH}), 130.7(\mathrm{CH}), 132.4,135.2(\mathrm{CCl}), 146.3,147.6(\mathrm{CNN}), 150.7 \mathrm{ppm}$, $\mathrm{CNO}_{2}$ could not be detected; $\mathrm{MS} m / z\left(I_{\mathrm{rel}}, \%\right): 512\left[\mathrm{M}^{+}\right](2), 449\left[\mathrm{M}-\mathrm{Cl}-\mathrm{N}_{2}\right]^{+}(1), 401[\mathrm{M}-\mathrm{PhCl}]^{+}(2), 138$ $[\mathrm{PhClNCH}]^{+}(100)$; HRMS $\left(\mathrm{ESI}^{+}\right) \mathrm{m} / z$ calcd for $\mathrm{C}_{20} \mathrm{H}_{17} \mathrm{~N}_{6} \mathrm{O}_{2} \mathrm{Cl}_{4}[\mathrm{M}+\mathrm{H}]^{+}$: 512.0078; found: 512.0075 .

Synthesis of 4-(4-Chlorophenyl)-1-[5-(dichloromethyl)-1-methyl-4-nitro-1H-pyrazol-3-yl]piperidin-4-ol (29a) (General method). To a suspension of azole $25 \mathrm{~g}(0.529 \mathrm{~g}, 1.0 \mathrm{mmol})$ in $\mathrm{MeOH}(10 \mathrm{~mL})$ at $-10{ }^{\circ} \mathrm{C}$, methylhydrazine $(0.921 \mathrm{~g}, 2.0 \mathrm{mmol})$ was added dropwise. After $2 \mathrm{~h}$, the solution was allowed reach r.t. and stirred for $1 \mathrm{~d}$. The solution was then concentrated and $10 \% \mathrm{HCl}(5 \mathrm{~mL})$ was added. The resulting precipitate was filtered off, washed with water, and purified by column chromatography using chloroform. Yield $0.311 \mathrm{~g}(74 \%)$, yellow solid, m.p. 95-96 ${ }^{\circ} \mathrm{C}$. IR (ATR) $v_{\max }=2951,1553,1484$, 1348, 1024, $746 \mathrm{~cm}^{-1} .{ }^{1} \mathrm{H}$ NMR $\left(400 \mathrm{MHz}, \mathrm{CDCl}_{3}\right) \delta=1.65(1 \mathrm{H}, \mathrm{s}, \mathrm{OH}), 1.78-1.85\left(2 \mathrm{H}, \mathrm{m}, \mathrm{CCH}_{2}\right), 2.28$ $\left(2 \mathrm{H}, \mathrm{ddd}, J=13.1 \mathrm{~Hz}, J=4.2 \mathrm{~Hz}, J=13.1 \mathrm{~Hz}, \mathrm{CCH}_{2}\right), 3.33(2 \mathrm{H}, \mathrm{ddd}, J=12.5 \mathrm{~Hz}, J=2.5 \mathrm{~Hz}, J=12.5 \mathrm{~Hz}$, $\left.\mathrm{NCH}_{2}\right), 3.51-3.59\left(2 \mathrm{H}, \mathrm{m}, \mathrm{NCH}_{2}\right), 4.12\left(3 \mathrm{H}, \mathrm{s}, \mathrm{NCH}_{3}\right), 7.34(2 \mathrm{H}, \mathrm{d}, J=8.7 \mathrm{~Hz}, \mathrm{H}$ Ar $), 7.47(2 \mathrm{H}, \mathrm{d}, J=8.7$ $\mathrm{Hz}, \mathrm{H}$ Ar), $7.88\left(1 \mathrm{H}, \mathrm{s}, \mathrm{CHCl}_{2}\right) \mathrm{ppm} ;{ }^{13} \mathrm{C} \mathrm{NMR}\left(100 \mathrm{MHz}, \mathrm{CDCl}_{3}\right) \delta=38.0\left(2 \times \mathrm{CH}_{2}\right), 39.7\left(\mathrm{NCH}_{3}\right), 46.0$ $\left(2 \times \mathrm{NCH}_{2}\right), 57.8\left(\mathrm{CHCl}_{2}\right), 71.1(\mathrm{HO}-\mathrm{C}), 120.4\left(\mathrm{CNO}_{2}\right), 126.1(\mathrm{CH}), 129.2(\mathrm{CH}), 133.0(\mathrm{CCl}), 137.9,146.5$, 152.8 (NC-pyr) ppm; MS m/z (I $\left.\mathrm{I}_{\text {rel }}, \%\right): 418\left[\mathrm{M}^{+}\right](3), 401[\mathrm{M}-\mathrm{OH}]^{+}(6), 383[\mathrm{M}-\mathrm{Cl}]^{+}(5), 367[\mathrm{M}-\mathrm{Cl}-\mathrm{O}]^{+}$ (4), $347[\mathrm{M}-\mathrm{Cl}-\mathrm{HCl}]^{+}(4), 100$ (100); $\mathrm{HRMS}\left(\mathrm{ESI}^{+}\right) \mathrm{m} / \mathrm{z}$ calcd for $\mathrm{C}_{16} \mathrm{H}_{18} \mathrm{~N}_{4} \mathrm{O}_{3} \mathrm{Cl}_{3}[\mathrm{M}+\mathrm{H}]^{+}: 419.0439$; found: 419.0437.

\{4-[5-(Dichloromethyl)-1-methyl-4-nitro-1H-pyrazol-3-yl]piperazin-1-yl\}(tetrahydrofuran-2-yl)methanone (29b). Same procedure as for 29a, using diene $25 \mathrm{~h}(0.502 \mathrm{~g}, 1.0 \mathrm{mmol})$ and DCM as the eluent. Yield $0.310 \mathrm{~g}(79 \%)$, yellowish oil. IR (ATR) $v_{\max }=2954,1647,1551,1341,1198,745 \mathrm{~cm}^{-1} .{ }^{1} \mathrm{H}$ NMR $(400$ $\left.\mathrm{MHz}_{2} \mathrm{CDCl}_{3}\right) \delta=1.86-2.12\left(3 \mathrm{H}, \mathrm{m}, \mathrm{CH}_{2}\right.$ Fur $), 2.25-2.35\left(1 \mathrm{H}, \mathrm{m}, \mathrm{CH}_{2}\right.$ Fur $), 3.17-3.31\left(4 \mathrm{H}, \mathrm{m}, \mathrm{NCH}_{2}\right)$, 3.63-3.73 (2H, m, NCH $), 3.79-3.98\left(4 \mathrm{H}, \mathrm{m}, \mathrm{NCH}_{2}, \mathrm{OCH}_{2}\right), 4.10\left(3 \mathrm{H}, \mathrm{s}, \mathrm{NCH}_{3}\right), 4.64(1 \mathrm{H}, \mathrm{dd}, J=5.6$ 
$\mathrm{Hz}, J=7.5 \mathrm{~Hz}, \mathrm{COCHO}), 7.84\left(1 \mathrm{H}, \mathrm{s}, \mathrm{CHCl}_{2}\right) \mathrm{ppm} ;{ }^{13} \mathrm{C} \mathrm{NMR}\left(100 \mathrm{MHz}, \mathrm{CDCl}_{3}\right) \delta=25.7\left(\mathrm{CH}_{2}\right), 28.4$ $\left(\mathrm{CH}_{2}\right), 39.7\left(\mathrm{NCH}_{3}\right), 41.6\left(\mathrm{NCH}_{2}\right), 45.0\left(\mathrm{NCH}_{2}\right), 49.6\left(\mathrm{NCH}_{2}\right), 50.0\left(\mathrm{NCH}_{2}\right), 57.6(\mathrm{CH}), 69.1\left(\mathrm{OCH}_{2}\right), 75.9$ $(\mathrm{OCH}), 120.5\left(\mathrm{CNO}_{2}\right), 138.1,152.1,170.1(\mathrm{C}=\mathrm{O}) \mathrm{ppm}$; MS m/z (I rel $\left._{1} \%\right): 391\left[\mathrm{M}^{+}\right](100), 374[\mathrm{M}-\mathrm{OH}]^{+}$

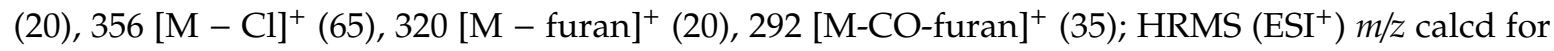
$\mathrm{C}_{14} \mathrm{H}_{20} \mathrm{~N}_{5} \mathrm{O}_{4} \mathrm{Cl}_{2}[\mathrm{M}+\mathrm{H}]^{+}$: 392.0887; found: 392.0889 .

1-(3-Chlorophenyl)-4-[5-(dichloromethyl)-1-methyl-4-nitro-1H-pyrazol-3-yl]piperazine (29c). Same proce-dure as for $29 \mathrm{a}$, using diene $25 \mathrm{i}(0.514 \mathrm{~g}, 1.0 \mathrm{mmol})$ and a mixture of petroleum ether and ethyl acetate (5: 1 ) as the eluent. Yield $0.299 \mathrm{~g}(74 \%)$, yellow solid, m.p. $162-163{ }^{\circ} \mathrm{C}$. IR (ATR) $v_{\max }$ $=2832,1552,1475,1338,936,737 \mathrm{~cm}^{-1} .{ }^{1} \mathrm{H}$ NMR $\left(400 \mathrm{MHz}, \mathrm{CDCl}_{3}\right) \delta=3.31-3.36\left(4 \mathrm{H}, \mathrm{m}, \mathrm{NCH}_{2}\right)$, 3.38-3.43 (4H, m, NCH 2$), 4.13\left(3 \mathrm{H}, \mathrm{s}, \mathrm{NCH}_{3}\right), 6.81-6.86(2 \mathrm{H}, \mathrm{m}, \mathrm{H} \mathrm{Ar}), 6.93(1 \mathrm{H}, \mathrm{t}, J=2.1 \mathrm{~Hz}, \mathrm{H} \mathrm{Ar})$, $7.18(1 \mathrm{H}, \mathrm{t}, J=8.0 \mathrm{~Hz}, \mathrm{H} \mathrm{Ar}), 7.87\left(1 \mathrm{H}, \mathrm{s}, \mathrm{CHCl}_{2}\right) \mathrm{ppm} ;{ }^{13} \mathrm{C} \mathrm{NMR}\left(100 \mathrm{MHz}, \mathrm{CDCl}_{3}\right) \delta=39.7\left(\mathrm{CH}_{3}\right)$, $48.5\left(\mathrm{NCH}_{2}\right), 49.5\left(\mathrm{NCH}_{2}\right), 57.7\left(\mathrm{CHCl}_{2}\right), 114.1(\mathrm{CH}), 116.1(\mathrm{CH}), 119.7(\mathrm{CH}), 120.5\left(\mathrm{CNO}_{2}\right), 130.1(\mathrm{CH})$, 135.0 (CCl), 138.0, 152.2 (CN), $152.3(\mathrm{CN}) \mathrm{ppm}$; MS m/z (I Irel $\left._{1} \%\right): 403\left[\mathrm{M}^{+}\right](45), 357\left[\mathrm{M}-\mathrm{NO}_{2}\right]^{+}(6)$, $322\left[\mathrm{M}-\mathrm{NO}_{2}-\mathrm{Cl}\right]^{+}(22), 138[\mathrm{PhClNCH}]^{+}(100)$; $\mathrm{HRMS}\left(\mathrm{ESI}^{+}\right) \mathrm{m} / z$ calcd for $\mathrm{C}_{15} \mathrm{H}_{17} \mathrm{~N}_{5} \mathrm{O}_{2} \mathrm{Cl}_{3}[\mathrm{M}+\mathrm{H}]^{+}$: 404.0442; found: 404.0444 .

Synthesis of 3-[4-(3-Chlorophenyl)piperazin-1-yl]-1-methyl-4-nitro-1H-pyrazole-5-carbaldehyde (30). Pyrazole 29c $(0.405 \mathrm{~g}, 1.0 \mathrm{mmol})$ was suspended in $10 \mathrm{~mL}$ sulfuric acid $(25 \%)$ and heated to $95-100{ }^{\circ} \mathrm{C}$ for $10 \mathrm{~h}$. After cooling to r.t., the solution was extracted with chloroform $(3 \times 10 \mathrm{~mL})$ and purified by column chromatography using petroleum ether - ethyl acetate (5: 1). The product was dried in vacuo. Yield $0.199 \mathrm{~g}(57 \%)$, yellow solid, m.p. $159-160^{\circ} \mathrm{C}$. IR (ATR) $v_{\max }=2827,1683,1546,1474,1236,775 \mathrm{~cm}^{-1}$. ${ }^{1} \mathrm{H}$ NMR $\left(400 \mathrm{MHz}, \mathrm{CDCl}_{3}\right) \delta=3.32-3.38\left(4 \mathrm{H}, \mathrm{m}, \mathrm{NCH}_{2}\right), 3.45-3.5\left(4 \mathrm{H}, \mathrm{m}, \mathrm{NCH}_{2}\right), 6.81-6.87(2 \mathrm{H}, \mathrm{m}, \mathrm{H}$ $\mathrm{Ph}), 6.93(1 \mathrm{H}, \mathrm{t}, J=2.0 \mathrm{~Hz}, \mathrm{H} \mathrm{Ph}), 7.19(1 \mathrm{H}, \mathrm{t}, J=8.1 \mathrm{~Hz}, \mathrm{H} \mathrm{Ph}), 10.43(1 \mathrm{H}, \mathrm{s}, \mathrm{CHO}) \mathrm{ppm} ;{ }^{13} \mathrm{C} \mathrm{NMR}(100$ $\left.\mathrm{MHz}, \mathrm{CDCl}_{3}\right) \delta=40.9\left(\mathrm{NCH}_{3}\right), 48.5\left(2 \times \mathrm{NCH}_{2}\right), 49.4\left(2 \times \mathrm{NCH}_{2}\right), 114.2(\mathrm{CH}), 116.1(\mathrm{CH}), 119.8(\mathrm{CH})$, $125.7\left(\mathrm{CNO}_{2}\right), 130.1(\mathrm{CH}), 135.0(\mathrm{CCl}), 135.2(\underline{\mathrm{CCHO}}), 151.9,152.1,181.8(\mathrm{CHO}) \mathrm{ppm}$; MS m/z (I $\left.\mathrm{rel}, \%\right)$ :

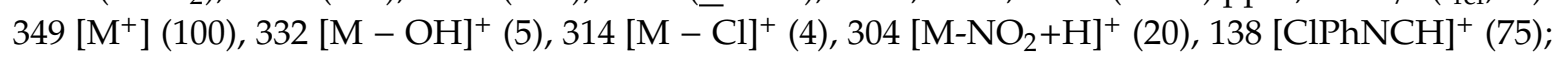
HRMS (ESI ${ }^{+}$) $m / z$ calcd for $\mathrm{C}_{15} \mathrm{H}_{17} \mathrm{~N}_{5} \mathrm{O}_{3} \mathrm{Cl}[\mathrm{M}+\mathrm{H}]^{+}$: 350.1014; found: 350.1016 .

Synthesis of 2-(1H-Benzotriazol-1-yl)-4-(dichloromethylidene)-3-nitro-4H-pyrido[1,2-a]pyrimidine (31a) (General method). To a solution of azole $24(0.437 \mathrm{~g}, 1.0 \mathrm{mmol})$ in THF $(10 \mathrm{~m}), 2$-aminopyridine $(0.282 \mathrm{~g}, 3.0 \mathrm{mmol})$ was added at r.t. The solution was stirred for $8 \mathrm{~h}$, then concentrated, and the residue treated with dilute $\mathrm{HCl}$ for $1 \mathrm{~h}$, filtered off and washed with water $(2 \times 10 \mathrm{~mL})$ and cold $\mathrm{MeOH}(5$ $\mathrm{mL})$. The product was dried in vacuo. Yield $0.304 \mathrm{~g}(81 \%)$, red solid, m.p. $158-160{ }^{\circ} \mathrm{C}$. IR $(\mathrm{KBr}) v_{\max }=$ $3082,1628,1552,1434,1307,1215 \mathrm{~cm}^{-1} .{ }^{1} \mathrm{H}$ NMR $\left(200 \mathrm{MHz}, \mathrm{DMSO}-\mathrm{d}_{6}\right) \delta=7.50-7.64(2 \mathrm{H}, \mathrm{m}, \mathrm{H} \mathrm{Ar})$, 7.64-7.75 (1H, m, H Bzt), 7.87 (1H, d, J = 8.3 Hz, H Ar), 7.90-7.95 (1H, m, H Bzt), $8.21(1 \mathrm{H}, \mathrm{d}, J=7.8$ $\mathrm{Hz}, \mathrm{H}$ Bzt), 8.22-8.32 (1H, m, H Bzt), $8.99\left(1 \mathrm{H}, \mathrm{dd}, J=6.8 \mathrm{~Hz}, 0.9 \mathrm{~Hz}, \mathrm{H}\right.$ Ar) ppm; ${ }^{13} \mathrm{C}$ NMR $(50 \mathrm{MHz}$, DMSO-d $\left._{6}\right) \delta=102.3\left(\mathrm{CCl}_{2}\right), 113.0(\mathrm{CH}), 119.2(\mathrm{CH}), 120.0(\mathrm{CH}), 121.3\left(\mathrm{CNO}_{2}\right), 123.7(\mathrm{CH}), 125.5(\mathrm{CH})$, 126.3, $129.6(\mathrm{CH}), 132.3,138.8(\mathrm{CH}), 143.6(\mathrm{CH}), 145.6,148.0,151.3 \mathrm{ppm} ; \mathrm{MS} \mathrm{m} / \mathrm{z}\left(\mathrm{I}_{\text {rel }}, \%\right): \mathrm{m} / \mathrm{z}(\%)=374$ [M $^{+}$] (1), 256 [M-benzotriazole] ${ }^{+}(14), 219$ (18), 119 [benzotriazole] (14), 92 (100); HRMS (ESI ${ }^{+}$) m/z calcd for $\mathrm{C}_{15} \mathrm{H}_{9} \mathrm{~N}_{6} \mathrm{O}_{2} \mathrm{Cl}_{2}[\mathrm{M}+\mathrm{H}]^{+}$: 375.0164; found: 375.0164 .

2-(1H-Benzotriazol-1-yl)-7-chloro-4-(dichloromethylidene)-3-nitro-4H-pyrido[1,2-a]pyrimidine (31b). Same procedure as for 31a, using 2-amino-5-chloropyridine $(0.386 \mathrm{~g}, 3.0 \mathrm{mmol})$ and heating the reaction mixture to $45{ }^{\circ} \mathrm{C}$. Yield $0.352 \mathrm{~g}(86 \%)$, orange solid, m.p. $182-184{ }^{\circ} \mathrm{C}$. IR $(\mathrm{KBr}) v_{\max }=3066,1505,1437$, $1318,1238,1222 \mathrm{~cm}^{-1} .{ }^{1} \mathrm{H}$ NMR $\left(200 \mathrm{MHz}, \mathrm{DMSO}_{-} \mathrm{d}_{6}\right) \delta=7.55(1 \mathrm{H}, \mathrm{t}, J=7.9 \mathrm{~Hz}, \mathrm{H}$ Bzt), $7.71(1 \mathrm{H}, \mathrm{t}, J=$ $8.1 \mathrm{~Hz}, \mathrm{H}$ Bzt), $7.86(1 \mathrm{H}, \mathrm{d}, J=9.3 \mathrm{~Hz}, \mathrm{H}$ pyr), $7.92(1 \mathrm{H}, \mathrm{d}, J=8.2 \mathrm{~Hz}, \mathrm{H}$ Bzt), $8.23(1 \mathrm{H}, \mathrm{d}, J=8.2 \mathrm{~Hz}, \mathrm{H}$ Bzt), $8.35(1 \mathrm{H}, \mathrm{dd}, J=9.4 \mathrm{~Hz}, J=2.3 \mathrm{~Hz}, \mathrm{H} \mathrm{pyr}), 9.35(1 \mathrm{H}, \mathrm{d}, J=2.3 \mathrm{~Hz}, \mathrm{H}$ pyr $)$ ppm; ${ }^{13} \mathrm{C}$ NMR (50 MHz, DMSO-d $\left.{ }_{6}\right) \delta=102.8\left(\mathrm{CCl}_{2}\right), 112.9(\mathrm{CH}), 119.9(\mathrm{CH}), 122.2\left(\mathrm{CNO}_{2}\right), 124.7(\mathrm{CCl}), 124.8(\mathrm{CH}), 125.6$ (CH), $129.6(\mathrm{CH}), 132.3,136.6(\mathrm{CH}), 143.1(\mathrm{CH}), 145.6,147.7(\mathrm{NC}), 150.6 \mathrm{ppm} ; \mathrm{MS} \mathrm{m} / \mathrm{z}\left(\mathrm{I}_{\text {rel }}, \%\right): 408\left[\mathrm{M}^{+}\right]$ (1), $380\left[\mathrm{M}-\mathrm{N}_{2}\right]^{+}(1), 373[\mathrm{M}-\mathrm{Cl}]^{+}(2), 334\left[\mathrm{M}-\mathrm{N}_{2}-\mathrm{NO}_{2}\right]^{+}$(7), 112 (100); HRMS (ESI ${ }^{+}$) m/z calcd for $\mathrm{C}_{15} \mathrm{H}_{8} \mathrm{~N}_{6} \mathrm{O}_{2} \mathrm{Cl}_{3}[\mathrm{M}+\mathrm{H}]^{+}$: 408.9769; found: 408.9768 . 
Synthesis of 4-(dichloromethylidene)-2-[4-(4-fluorophenyl)piperazin-1-yl]-3-nitro-4H-pyrido[1,2-a]pyrimi- dine (32a) (General method). To a solution of pyrimidine 31a $(0.375 \mathrm{~g}, 1 \mathrm{mmol})$ in $\mathrm{MeOH}(10 \mathrm{~mL})$ 1-(4-fluorophenyl)piperazine $(0.216 \mathrm{~g}, 1.2 \mathrm{mmol})$ was added and the mixture stirred at $40{ }^{\circ} \mathrm{C}$ for $5 \mathrm{~h}$. Subsequently, the mixture was cooled to $0{ }^{\circ} \mathrm{C}$ and treated with dilute $\mathrm{HCl}$ for $1 \mathrm{~h}$. The precipitate was filtered off and washed with water $(2 \times 5 \mathrm{~mL})$ and cold $\mathrm{MeOH}(5 \mathrm{~mL})$. The product was dried in vacuo. Yield $0.423 \mathrm{~g}(97 \%)$, yellow solid, m.p. $126-127^{\circ} \mathrm{C} . \mathrm{IR}(\mathrm{KBr}) v_{\max }=1639,1551,1504,1253$, $1145,933 \mathrm{~cm}^{-1} .{ }^{1} \mathrm{H}$ NMR $\left(200 \mathrm{MHz}, \mathrm{DMSO}-\mathrm{d}_{6}\right) \delta=3.15-3.50\left(4 \mathrm{H}, \mathrm{m}, \mathrm{NCH}_{2}\right), 3.78-4.31\left(4 \mathrm{H}, \mathrm{m}, \mathrm{NCH}_{2}\right)$, 6.78-7.18 (5H, m, H Pyr, 4H Ph-F), $7.31(1 \mathrm{H}, \mathrm{d}, J=8.6 \mathrm{~Hz}, \mathrm{H}$ pyr), $7.94(1 \mathrm{H}, \mathrm{ddd}, J=8.6 \mathrm{~Hz}, J=1.3 \mathrm{~Hz}$, $J=7.3 \mathrm{~Hz}, \mathrm{H}$ pyr), $8.60(1 \mathrm{H}, \mathrm{dd}, J=7.2 \mathrm{~Hz}, J=1.3 \mathrm{~Hz}, \mathrm{H}$ pyr $)$ ppm; ${ }^{13} \mathrm{C}$ NMR $\left(50 \mathrm{MHz}, \mathrm{DMSO}-\mathrm{d}_{6}\right)$ $\delta=49.5\left(4 \times \mathrm{NCH}_{2}\right), 94.9\left(\mathrm{CCl}_{2}\right), 114.7(\mathrm{CH}), 115.6\left(J_{\mathrm{C}, \mathrm{F}}=21.6 \mathrm{~Hz}, 2 \times \mathrm{CH} \mathrm{Ph}\right), 118.1\left(J_{\mathrm{C}, \mathrm{F}}=7.9 \mathrm{~Hz}\right.$, $2 \times \mathrm{CH} \mathrm{Ph}), 120.5\left(\mathrm{CNO}_{2}\right), 122.7(\mathrm{CH}), 128.2\left(\mathrm{CCCl}_{2}\right), 137.1(\mathrm{CH}), 142.0(\mathrm{CH}), 147.3(\mathrm{NC} \mathrm{Ph}), 151.5$, $156.6\left(J_{\mathrm{C}, \mathrm{F}}=235.9 \mathrm{~Hz}, \mathrm{CF}\right), 156.1(\mathrm{NCN}) \mathrm{ppm} ; \mathrm{MS} \mathrm{m} / z\left(I_{\mathrm{rel}}, \%\right): 435\left[^{+} \mathrm{M}^{+}\right](7), 256[\mathrm{M}-\mathrm{morph}-\mathrm{Ph}-\mathrm{F}]^{+}(10)$, 179 [Morph-Ph-F] $]^{+}$(15), 95 (100); HRMS (ESI ${ }^{+}$) $m / z$ calcd for $\mathrm{C}_{19} \mathrm{H}_{17} \mathrm{~N}_{5} \mathrm{O}_{2} \mathrm{Cl}_{2} \mathrm{~F}[\mathrm{M}+\mathrm{H}]^{+}: 436.0738$; found: 436.0736 .

7-Chloro-4-(dichloromethylidene)-2-[4-(4-fluorophenyl)piperazin-1-yl]-3-nitro-4H-pyrido[1,2-a]pyrimidine (32b). Same procedure as for 32a, using compound $31 \mathbf{b}(0.410 \mathrm{~g}, 1.0 \mathrm{mmol})$ but without adding $\mathrm{HCl}$. Yield $0.447 \mathrm{~g}(95 \%)$, yellow solid, m.p. $141-142{ }^{\circ} \mathrm{C}$. IR $(\mathrm{KBr}) v_{\max }=1635,1563,1493,1373,1237,827$ $\mathrm{cm}^{-1} .{ }^{1} \mathrm{H}$ NMR $\left(200 \mathrm{MHz}, \mathrm{DMSO}_{6}\right) \delta=3.14-3.29\left(4 \mathrm{H}, \mathrm{m}, \mathrm{NCH}_{2}\right), 3.50-4.33\left(4 \mathrm{H}, \mathrm{m}, \mathrm{NCH}_{2}\right), 6.95-7.14$ $(4 \mathrm{H}, \mathrm{m}, \mathrm{H} \mathrm{Ph}), 7.31(1 \mathrm{H}, \mathrm{d}, J=9.4 \mathrm{~Hz}, \mathrm{H}$ pyr), $7.99(1 \mathrm{H}, \mathrm{dd}, J=9.5 \mathrm{~Hz}, J=2.3 \mathrm{~Hz}, \mathrm{H} \mathrm{pyr}) ; 8.92(1 \mathrm{H}, \mathrm{d}, J$ $=8.9 \mathrm{~Hz}, \mathrm{H}$ pyr $)$ ppm; ${ }^{13} \mathrm{C} \mathrm{NMR}\left(50 \mathrm{MHz}, \mathrm{DMSO}-\mathrm{d}_{6}\right) \delta=49.4\left(4 \times \mathrm{NCH}_{2}\right), 94.7\left(\mathrm{CCl}_{2}\right), 115.3\left(J_{\mathrm{C}, \mathrm{F}}=\right.$ $21.9 \mathrm{~Hz}, 2 \times \mathrm{FCC} H), 117.6\left(\mathrm{~J}_{\mathrm{C}, \mathrm{F}}=7.7 \mathrm{~Hz}, 2 \times \mathrm{CH} \mathrm{Ph}\right), 120.1(\mathrm{CCl}), 121.1\left(\mathrm{CNO}_{2}\right), 123.9(\mathrm{CH}), 127.6$, $134.7(\mathrm{NCH}), 141.8(\mathrm{CH}), 147.3\left(J_{\mathrm{C}, \mathrm{F}}=1.8 \mathrm{~Hz}, \mathrm{NC} \mathrm{Ph}\right), 150.4,156.2\left(J_{\mathrm{C}, \mathrm{F}}=236.4 \mathrm{~Hz}, \mathrm{CF}\right), 156.0 \mathrm{ppm} ; \mathrm{MS}$

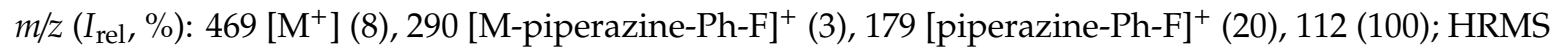
$\left(\mathrm{ESI}^{+}\right) \mathrm{m} / z$ calcd for $\mathrm{C}_{19} \mathrm{H}_{16} \mathrm{~N}_{5} \mathrm{O}_{2} \mathrm{Cl}_{3} \mathrm{~F}[\mathrm{M}+\mathrm{H}]^{+}: 470.0348$; found: 470.0350 .

Synthesis of Ethyl \{[4-(dichloromethylidene)-3-nitro-4H-pyrido[1,2-a]pyrimidin-2-yl]sulfanyl\}acetate (33a) (General method). To a solution of pyrimidine 31a $(0.375 \mathrm{~g}, 1.0 \mathrm{mmol})$ in EtOH $(10 \mathrm{~mL})$, ethyl 2-mercaptoacetate $(0.240 \mathrm{~g}, 2.0 \mathrm{mmol})$ and sodium ethanolate $(0.204 \mathrm{~g}, 3.0 \mathrm{mmol})$ were added. The solution was stirred at r.t. for $3 \mathrm{~d}$. Subsequently, dilute $\mathrm{HCl}(5 \mathrm{~mL})$ was added under stirring for 30 min. The resulting precipitate was filtered off, washed with water $(2 \times 5 \mathrm{~mL})$ and cold $\mathrm{MeOH}(3 \mathrm{~mL})$, and dried in vacuo. Yield $0.365 \mathrm{~g}(97 \%)$.

Alternative synthesis of pyrimidine 33a from nitrodiene 18. To a solution of nitrobutadiene $18(0.355 \mathrm{~g}, 1.0$ $\mathrm{mmol})$ in $\mathrm{MeOH}(10 \mathrm{~mL})$ 2-aminopyridine $(0.282 \mathrm{~g}, 3.0 \mathrm{mmol})$ was added. After stirring at r.t. for $1 \mathrm{~d}$, the solution was concentrated and the resulting precipitate filtered off, washed with water $(2 \times 3 \mathrm{~mL})$, cold $\mathrm{MeOH}(3 \mathrm{~mL})$ and dried in vacuo. Yield $0.177 \mathrm{~g}(47 \%)$ of 33a, orange solid, m.p. $147-148{ }^{\circ} \mathrm{C}$. IR $\left(\right.$ ATR) $\nu_{\max }=1731,1597,1451,1202,1141,764 \mathrm{~cm}^{-1} .{ }^{1} \mathrm{H} \mathrm{NMR}\left(400 \mathrm{MHz}, \mathrm{CDCl}_{3}\right) \delta=1.27(3 \mathrm{H}, \mathrm{t}, J=7.1$ $\left.\mathrm{Hz}, \mathrm{OCH}_{2} \mathrm{CH}_{3}\right) ; 3.85\left(2 \mathrm{H}, \mathrm{s}, \mathrm{SCH}_{2}\right), 4.20\left(2 \mathrm{H}, \mathrm{q}, J=7.1 \mathrm{~Hz}, \mathrm{OCH}_{2}\right), 7.00(1 \mathrm{H}, \mathrm{ddd}, J=1.3 \mathrm{~Hz}, J=6.9 \mathrm{~Hz}$, $J=6.9 \mathrm{~Hz}, \mathrm{CH}), 7.23(1 \mathrm{H}, \mathrm{ddd}, J=1.2 \mathrm{~Hz}, J=0.6 \mathrm{~Hz}, J=9.0 \mathrm{~Hz}, \mathrm{CH}), 7.72(1 \mathrm{H}, \mathrm{ddd}, J=1.7 \mathrm{~Hz}, J=7.1$ $\mathrm{Hz}, J=8.8 \mathrm{~Hz}, \mathrm{CH}), 8.04(1 \mathrm{H}, \mathrm{ddd}, J=1.6 \mathrm{~Hz}, J=0.7 \mathrm{~Hz}, J=6.9 \mathrm{~Hz}, \mathrm{NCH}) \mathrm{ppm} ;{ }^{13} \mathrm{C} \mathrm{NMR}(100 \mathrm{MHz}$, $\left.\mathrm{CDCl}_{3}\right) \delta=14.2\left(\mathrm{CH}_{3}\right), 33.7\left(\mathrm{SCH}_{2}\right), 61.5\left(\mathrm{OCH}_{2}\right), 114.5(\mathrm{CH}), 117.1\left(\mathrm{CCl}_{2}\right), 123.0(\mathrm{CH}), 125.1\left(\mathrm{CNO}_{2}\right)$, $135.6(\mathrm{NCH}), 140.0(\mathrm{CH}), 149.7(\mathrm{NCN}), 164.1(\mathrm{SCN}), 169.3(\mathrm{C}=\mathrm{O}) \mathrm{ppm}, \mathrm{NCCHCl}_{2}$ could not be detected; MS $m / z\left(I_{\text {rel }} \%\right): 375\left[\mathrm{M}^{+}\right](10), 340[\mathrm{M}-\mathrm{Cl}]^{+}(9), 288\left[\mathrm{M}-\mathrm{CH}_{2} \mathrm{CO}_{2} \mathrm{Et}\right]^{+}(25), 209\left[\mathrm{M}-\mathrm{HNO}_{2}-\mathrm{SCH}_{2} \mathrm{CO}_{2} \mathrm{Et}\right]^{+}$ (95), 149 (100); HRMS (ESI ${ }^{+}$) m/z calcd for $\mathrm{C}_{13} \mathrm{H}_{11} \mathrm{~N}_{3} \mathrm{O}_{4} \mathrm{Cl}_{2} \mathrm{SNa}[\mathrm{M}+\mathrm{Na}]^{+}$: 397.9745; found: 397.9746.

Ethyl \{[7-chloro-4-(dichloromethylidene)-3-nitro-4H-pyrido[1,2-a]pyrimidin-2-yl]sulfanyl\}acetate (33b). Same procedure as for 33a,but starting from compounds $\mathbf{3 1 b}$ (Yield $0.382 \mathrm{~g}, 93 \%$ ), or $\mathbf{1 8}$ (Yield 0.226 g, 55\%), orange solid, m.p. $171-172{ }^{\circ} \mathrm{C}$. IR (ATR) $v_{\max }=3074,1727,1626,1489,1235,741 \mathrm{~cm}^{-1} .{ }^{1} \mathrm{H}$ NMR $(200$ $\left.\mathrm{MHz} \mathrm{CDCl}_{3}\right) \delta=1.27\left(3 \mathrm{H}, \mathrm{t}, J=7.1 \mathrm{~Hz}, \mathrm{CH}_{3}\right), 3.83\left(2 \mathrm{H}, \mathrm{s}, \mathrm{SCH}_{2}\right), 4.19\left(2 \mathrm{H}, \mathrm{q}, J=7.2 \mathrm{~Hz}, \mathrm{OCH}_{2}\right), 7.17$ $(1 \mathrm{H}, \mathrm{dd}, J=9.4 \mathrm{~Hz}, J=0.5 \mathrm{~Hz}, \mathrm{CH}), 7.63(1 \mathrm{H}, \mathrm{dd}, J=9.5 \mathrm{~Hz}, J=2.3 \mathrm{~Hz}, \mathrm{CH}), 8.05(1 \mathrm{H}, \mathrm{dd}, J=2.3 \mathrm{~Hz}, J=$ $0.6 \mathrm{~Hz}, \mathrm{NCH}) \mathrm{ppm} ;{ }^{13} \mathrm{C} \mathrm{NMR}\left(50 \mathrm{MHz}, \mathrm{CDCl}_{3}\right) \delta=14.3\left(\mathrm{CH}_{3}\right), 33.6\left(\mathrm{SCH}_{2}\right), 61.6\left(\mathrm{OCH}_{2}\right), 117.5\left(\mathrm{CCl}_{2}\right)$, 
121.4 $\left(\mathrm{CNO}_{2}\right), 122.2(\mathrm{CCl}), 123.2(\mathrm{CH}), 124.4(\mathrm{NC}), 133.0(\mathrm{CH}), 140.5(\mathrm{CH}), 148.2,163.7(\mathrm{SC}), 169.2(\mathrm{C}=\mathrm{O})$ ppm; MS m/z (I $\left.I_{\text {rel }}, \%\right): 409\left[\mathrm{M}^{+}\right](10), 373[\mathrm{M}-\mathrm{HCl}]^{+}(18), 363\left[\mathrm{M}-\mathrm{NO}_{2}\right]^{+}(15), 321\left[\mathrm{M}-\mathrm{CH}_{3} \mathrm{CO}_{2} \mathrm{Et}\right]^{+}(45)$, 242 (100); HRMS (ESI ${ }^{+}$) $\mathrm{m} / z$ calcd for $\mathrm{C}_{13} \mathrm{H}_{10} \mathrm{~N}_{3} \mathrm{O}_{4} \mathrm{Cl}_{3} \mathrm{SNa}[\mathrm{M}+\mathrm{Na}]^{+}: 431.9355$; found: 431.9357.

Synthesis of N-[1-(benzylsulfanyl)-3,4,4-trichloro-2-nitrobuta-1,3-dien-1-yl]naphthalen-1-amine (35a) (General method). To a solution of diene $34 \mathrm{a}(0.359 \mathrm{~g}, 1.0 \mathrm{mmol})$ in $\mathrm{MeOH}(10 \mathrm{~mL})$ at $-10^{\circ} \mathrm{C}$, naphthalen-1-amine $(0.315 \mathrm{~g}, 2.2 \mathrm{mmol})$ was added. The solution was kept at $-10{ }^{\circ} \mathrm{C}$ for $2 \mathrm{~h}$ and then allowed to warm up to r.t. for $8 \mathrm{~h}$. Subsequently, the solution was concentrated and the resulting precipitate was filtered off, washed with water $(2 \times 5 \mathrm{~mL})$ and cold $\mathrm{MeOH}(5 \mathrm{~mL})$, and dried in vacuo. Yield $0.428 \mathrm{~g}(92 \%)$, green-yellow solid, m.p. $132-133^{\circ} \mathrm{C}$. IR (ATR) $v_{\max }=1536,1332,1149,939,768,702 \mathrm{~cm}^{-1} .{ }^{1} \mathrm{H} \mathrm{NMR}$ $\left(400 \mathrm{MHz}, \mathrm{CDCl}_{3}\right) \delta=3.47\left(2 \mathrm{H}, \mathrm{q}, J=12.4 \mathrm{~Hz}, \mathrm{SCH}_{2}\right), 6.86(2 \mathrm{H}, \mathrm{dd}, J=7.7 \mathrm{~Hz}, J=1.7 \mathrm{~Hz}, \mathrm{H} \mathrm{Ph})$, 7.14-7.25 (3H, m, H Ar), 7.57 (1H, dd, J = 7.8 Hz, J = 7.8 Hz, H Ph), 7.59-7.65 (2H, m, H Ph), $7.73(1 \mathrm{H}, \mathrm{d}$, $J=7.4 \mathrm{~Hz}, \mathrm{H} \mathrm{Ar}), 7.93(1 \mathrm{H}, \mathrm{d}, J=8.2 \mathrm{~Hz}, \mathrm{H} \mathrm{Ar}), 7.94-7.99(2 \mathrm{H}, \mathrm{m}, \mathrm{H} \mathrm{Ar}), 13.32(1 \mathrm{H}, \mathrm{s}, \mathrm{NH}) \mathrm{ppm} ;{ }^{13} \mathrm{C}$ $\operatorname{NMR}\left(100 \mathrm{MHz}, \mathrm{CDCl}_{3}\right) \delta=38.5\left(\mathrm{CH}_{2}\right), 121.5(\mathrm{CH}), 122.0\left(\mathrm{CNO}_{2}\right), 122.9(\mathrm{CH}), 123.9\left(\mathrm{CCl}_{2}\right), 125.4(\mathrm{CH})$, 127.2 $(\mathrm{CH}), 127.8(\mathrm{CH}), 128.0(\mathrm{CCl}), 128.2(\mathrm{CH}), 128.5,128.6(\mathrm{CH}), 128.7(2 \times \mathrm{CH}), 128.7(\mathrm{CH}), 128.9(2 \times$ $\mathrm{CH}), 133.4,134.1,134.2,159.7$ (NCS) ppm; MS m/z (I $\left.\mathrm{I}_{\text {rel }}, \%\right): 464\left[\mathrm{M}^{+}\right](1), 447[\mathrm{M}-\mathrm{OH}]^{+}(1), 418[\mathrm{M}-$ $\left.\mathrm{NO}_{2}\right]^{+}(1), 127$ [naphthalene] $]^{+}(11), 91\left[\mathrm{PhCH}_{2}\right]^{+}(100)$; HRMS (ESI $\left.{ }^{+}\right) \mathrm{m} / z$ calcd for $\mathrm{C}_{21} \mathrm{H}_{16} \mathrm{~N}_{2} \mathrm{O}_{2} \mathrm{Cl}_{3} \mathrm{~S}[\mathrm{M}$ $+\mathrm{H}]^{+}$: 464.9993; found: 464.9991 .

$N$-\{3,4,4-Trichloro-1-[(4-chlorophenyl)sulfanyl]-2-nitrobuta-1,3-dien-1-yl\}naphthalen-1-amine (35b). Same procedure as for 33a, but starting from 34b. Yield $0.423 \mathrm{~g}(87 \%)$, green-yellow solid, m.p. $185-186{ }^{\circ} \mathrm{C}$. IR (ATR) $v_{\max }=1538,1472,1345,1161,824,767 \mathrm{~cm}^{-1} .{ }^{1} \mathrm{H} \mathrm{NMR}\left(400 \mathrm{MHz}, \mathrm{CDCl}_{3}\right) \delta=6.68(2 \mathrm{H}, \mathrm{d}, J=$ $8.8 \mathrm{~Hz}, \mathrm{H} \mathrm{Ar}), 6.71(2 \mathrm{H}, \mathrm{d}, J=8.8 \mathrm{~Hz}, \mathrm{H} \mathrm{Ar}), 7.33-7.43(3 \mathrm{H}, \mathrm{m}, \mathrm{H} \mathrm{Ar}), 7.48(1 \mathrm{H}, \mathrm{ddd}, J=6.8 \mathrm{~Hz}, J=$ $1.2 \mathrm{~Hz}, J=8.1 \mathrm{~Hz}, \mathrm{H} \mathrm{Ar}), 7.55(1 \mathrm{H}, \mathrm{d}, J=8.5 \mathrm{~Hz}, \mathrm{H} \mathrm{Ar}), 7.71-7.80(2 \mathrm{H}, \mathrm{m}, \mathrm{H} \mathrm{Ar}), 11.96(1 \mathrm{H}, \mathrm{s}, \mathrm{NH})$ ppm; ${ }^{13} \mathrm{C} \mathrm{NMR}\left(100 \mathrm{MHz}, \mathrm{CDCl}_{3}\right) \delta=121.4,121.3(\mathrm{CH}), 123.8\left(\mathrm{CNO}_{2}\right), 124.6(\mathrm{CH}), 124.9(\mathrm{CH}), 125.6$, $126.8(\mathrm{CH}), 126.9(\mathrm{CH}), 128.2(\mathrm{CH}), 128.4,128.5,128.7(\mathrm{CH}), 129.1(2 \times \mathrm{CH}), 133.2,133.6,134.3(2 \times \mathrm{CH})$,

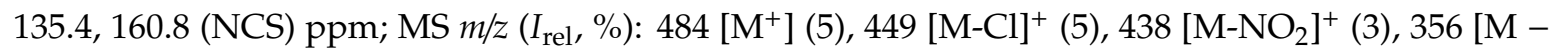
naphthalene] ${ }^{+}(2), 143$ [naphthylamine] $^{+}(60), 127$ [naphthalene] $^{+}(100) ; \mathrm{HRMS}\left(\mathrm{ESI}^{+}\right) \mathrm{m} / \mathrm{z}$ calcd for $\mathrm{C}_{20} \mathrm{H}_{13} \mathrm{~N}_{2} \mathrm{O}_{2} \mathrm{Cl}_{4} \mathrm{~S}[\mathrm{M}+\mathrm{H}]^{+}$: 484.9446; found: 484.9443 .

Synthesis of 2-(Benzylsulfanyl)-4-(dichloromethyl)-3-nitrobenzo[h]quinoline (36a) (General method). To a solution of diene $35 \mathrm{a}(0.466 \mathrm{~g}, 1.0 \mathrm{mmol})$ in chloroform $(10 \mathrm{~mL})$ at $0{ }^{\circ} \mathrm{C}$, triethylamine $(0.202 \mathrm{~g}, 2.0$ mmol) was added. The solution was kept at $0{ }^{\circ} \mathrm{C}$ for $2 \mathrm{~h}$ and then allowed to warm to r.t. for $3 \mathrm{~h}$. Subsequently, the solution was concentrated and diluted with $5 \mathrm{~mL} \mathrm{MeOH}$. The resulting precipitate was filtered off, washed with $10 \%$ aq. $\mathrm{HCl}(2 \times 5 \mathrm{~mL})$, water $(2 \times 5 \mathrm{~mL})$ and cold $\mathrm{MeOH}(5 \mathrm{~mL})$, and dried in vacuo to give benzoquinoline 36a. Yield $0.326 \mathrm{~g}(76 \%)$, yellow solid, m.p. $173-174{ }^{\circ} \mathrm{C}$. IR (ATR) $v_{\max }=3033,1541,1336,1198,828,711 \mathrm{~cm}^{-1} .{ }^{1} \mathrm{H} \mathrm{NMR}\left(400 \mathrm{MHz}, \mathrm{CDCl}_{3}\right) \delta=4.78\left(2 \mathrm{H}, \mathrm{s}, \mathrm{SCH}_{2}\right)$, $7.12\left(1 \mathrm{H}, \mathrm{s}, \mathrm{CHCl}_{2}\right), 7.27-7.36(3 \mathrm{H}, \mathrm{m}, \mathrm{H} \mathrm{Ph}), 7.48-7.52(2 \mathrm{H}, \mathrm{m}, \mathrm{H} \mathrm{Ph}), 7.57(1 \mathrm{H}, \mathrm{ddd}, J=7.2 \mathrm{~Hz}, J=$ $1.1 \mathrm{~Hz}, J=7.9 \mathrm{~Hz}, \mathrm{H} \mathrm{Ar}), 7.79(1 \mathrm{H}, \mathrm{ddd}, J=7.3 \mathrm{~Hz}, J=1.4 \mathrm{~Hz}, J=7.3 \mathrm{~Hz}, \mathrm{H} \mathrm{Ar}), 7.95(2 \mathrm{H}, \mathrm{d}, J=9.0$ $\mathrm{Hz}, \mathrm{H} \mathrm{Ar}), 8.64(1 \mathrm{H}, \mathrm{d}, J=9.4 \mathrm{~Hz}, \mathrm{H} \mathrm{Ar}), 9.18(1 \mathrm{H}, \mathrm{dd}, J=7.6 \mathrm{~Hz}, J=0.8 \mathrm{~Hz}, \mathrm{H} \mathrm{Ar}) \mathrm{ppm} ;{ }^{13} \mathrm{C} \mathrm{NMR}$ $\left(100 \mathrm{MHz}, \mathrm{CDCl}_{3}\right) \delta=35.8\left(\mathrm{SCH}_{2}\right), 63.4\left(\mathrm{CHCl}_{2}\right), 119.2,122.3(\mathrm{CH}), 125.4(\mathrm{CH}), 127.7(\mathrm{CH}), 127.9(\mathrm{CH})$, $128.0(\mathrm{CH}), 128.5(\mathrm{CH}), 128.7(2 \times \mathrm{CH}), 129.0(2 \times \mathrm{CH}), 130.1(\mathrm{CH}), 130.4,134.2,134.8,136.1,139.4,147.7$, $\left.149.8 \mathrm{ppm} ; \mathrm{MS} \mathrm{m} / \mathrm{z}\left(\mathrm{I}_{\mathrm{rel}}, \%\right): 428\left[\mathrm{M}^{+}\right](2), 344\left[\mathrm{M}-\mathrm{CH}_{2} \mathrm{Cl}_{2}\right]^{+}(2), 91\left[\mathrm{PhCH}_{2}\right]^{+}(100) ; \mathrm{HRMS}_{(\mathrm{ESI}}{ }^{+}\right) \mathrm{m} / \mathrm{z}$ calcd for $\mathrm{C}_{21} \mathrm{H}_{14} \mathrm{~N}_{2} \mathrm{O}_{2} \mathrm{Cl}_{2} \mathrm{SNa}[\mathrm{M}+\mathrm{Na}]^{+}$: 451.0051; found: 451.0050 .

2-[(4-Chlorophenyl)sulfanyl]-4-(dichloromethyl)-3-nitrobenzo[h]quinoline (36b). Same procedure as for 36a, but starting from $35 \mathrm{~b}$. Yield $0.382 \mathrm{~g}(85 \%)$, yellow solid, m.p. $162-163^{\circ} \mathrm{C}$. IR (ATR) $v_{\max }=3073,1572$, $1541,1388,1028,739 \mathrm{~cm}^{-1} .{ }^{1} \mathrm{H}$ NMR $\left(400 \mathrm{MHz}, \mathrm{CDCl}_{3}\right) \delta=7.19\left(1 \mathrm{H}, \mathrm{s}, \mathrm{CHCl}_{2}\right), 7.53(2 \mathrm{H}, \mathrm{d}, J=8.6 \mathrm{~Hz}$, $\mathrm{H} \mathrm{Ph}), 7.58(1 \mathrm{H}, \mathrm{ddd}, J=7.1 \mathrm{~Hz}, J=1.3 \mathrm{~Hz}, J=8.3 \mathrm{~Hz}, \mathrm{H} \mathrm{Ph}), 7.63(2 \mathrm{H}, \mathrm{d}, J=8.6 \mathrm{~Hz}, \mathrm{H} \mathrm{Ph}), 7.72(1 \mathrm{H}$, ddd $, J=7.0 \mathrm{~Hz}, J=1.1 \mathrm{~Hz}, J=8.1 \mathrm{~Hz}, \mathrm{H} \mathrm{Ph}), 7.88(1 \mathrm{H}, \mathrm{d}, J=7.9 \mathrm{~Hz}, \mathrm{H} \mathrm{Ph}), 7.92(1 \mathrm{H}, \mathrm{d}, J=9.3 \mathrm{~Hz}, \mathrm{H} \mathrm{Ph})$, $8.32(1 \mathrm{H}, \mathrm{d}, J=8.3 \mathrm{~Hz}, \mathrm{H} \mathrm{Ph}), 8.61(1 \mathrm{H}, \mathrm{d}, J=9.3 \mathrm{~Hz}, \mathrm{H} \mathrm{Ph}) \mathrm{ppm} ;{ }^{13} \mathrm{C} \mathrm{NMR}\left(100 \mathrm{MHz}, \mathrm{CDCl}_{3}\right) \delta=63.4$ $\left(\mathrm{CHCl}_{2}\right), 119.8,122.0(\mathrm{CH}), 125.3(\mathrm{CH}), 127.2,127.8(\mathrm{CH}), 128.0(\mathrm{CH}), 129.1(\mathrm{CH}), 129.6(2 \times \mathrm{CH}), 130.1$ 
(CH), 130.4 (SC Ph), $134.0(\mathrm{CCl}), 135.4,136.4\left(\mathrm{Cl}_{2} \mathrm{CHC}\right), 137.5(2 \times \mathrm{CH}), 138.9\left(\mathrm{CNO}_{2}\right), 147.8(\mathrm{NC}), 149.8$ (NCS) ppm; MS m/z (I rel, \%): $448\left[\mathrm{M}^{+}\right](28), 413[\mathrm{M}-\mathrm{Cl}]^{+}(7), 364\left[\mathrm{M}-\mathrm{CHCl}_{2}-\mathrm{NO}_{2}+\mathrm{H}\right]^{+}(15), 175(100)$, $111\left[_{\mathrm{PhCl}}{ }^{+}(55)\right.$; HRMS (ESI $\left.{ }^{+}\right) \mathrm{m} / z$ calcd for $\mathrm{C}_{20} \mathrm{H}_{11} \mathrm{~N}_{2} \mathrm{O}_{2} \mathrm{Cl}_{3} \mathrm{SNa}[\mathrm{M}+\mathrm{Na}]^{+}$: 470.9505; found: 470.9504 .

Synthesis of 2-(Benzylsulfinyl)-4-(dichloromethyl)-3-nitrobenzo[h]quinoline (37a) (General method). To a solution of quinoline $36 \mathrm{a}(0.429 \mathrm{~g}, 1.0 \mathrm{mmol})$ in chloroform $(5 \mathrm{~mL})$ and glacial acetic acid $(2 \mathrm{~mL})$ at $0{ }^{\circ} \mathrm{C}$, hydrogen peroxide $(1.13 \mathrm{~g}, 10.0 \mathrm{mmol}, 30 \%$ aq. $)$ was added dropwise. After $3 \mathrm{~h}$ at $0{ }^{\circ} \mathrm{C}$, the solution was allowed to reach r.t. and stirred additionally for $2 \mathrm{~d}$. The solution was then extracted with chloroform $(3 \times 30 \mathrm{~mL})$, washed with water $(2 \times 50 \mathrm{~mL})$, dried with $\mathrm{CaCl}_{2}$ and dried in vacuo. Column chromatography was carried out with a solvent ratio of 2: 1 (petroleum ether-ethyl acetate). Yield $0.396 \mathrm{~g}(89 \%)$, orange solid, m.p. $138-139{ }^{\circ} \mathrm{C}$. IR (ATR) $v_{\max }=1697,1533,1353,1076,830,695$ $\mathrm{cm}^{-1} .{ }^{1} \mathrm{H}$ NMR $\left(400 \mathrm{MHz}, \mathrm{CDCl}_{3}\right) \delta=4.57\left(2 \mathrm{H}, \mathrm{q}, J=12.7 \mathrm{~Hz}, \mathrm{SCH}_{2}\right), 7.25-7.31\left(6 \mathrm{H}\right.$ in all, $\mathrm{m}, \mathrm{CHCl}_{2}$ and 5H Ph overlapped), 7.81-7.92 (2H, m, H Ar), $8.02(1 \mathrm{H}, \mathrm{dd}, J=7.5 \mathrm{~Hz}, J=1.5 \mathrm{~Hz}, \mathrm{H} \mathrm{Ar}), 8.15(1 \mathrm{H}, \mathrm{d}$, $J=9.4 \mathrm{~Hz}, \mathrm{H} \mathrm{Ar}), 8.76(1 \mathrm{H}, \mathrm{d}, J=9.4 \mathrm{~Hz}, \mathrm{H} \mathrm{Ar}), 9.31(1 \mathrm{H}, \mathrm{dd}, J=7.7 \mathrm{~Hz}, J=1.0 \mathrm{~Hz}, \mathrm{H} \mathrm{Ar}) \mathrm{ppm} ;{ }^{13} \mathrm{C}$ $\operatorname{NMR}\left(100 \mathrm{MHz}, \mathrm{CDCl}_{3}\right) \delta=62.2\left(\mathrm{CH}_{2}\right), 62.5\left(\mathrm{CHCl}_{2}\right), 122.0(\mathrm{CH}), 123.3,126.2(\mathrm{CH}), 128.0(\mathrm{CH}), 128.6$ $(\mathrm{CH}), 128.7(\mathrm{CH}), 128.8(2 \times \mathrm{CH}), 129.5,130.4(2 \times \mathrm{CH}), 130.5,131.1(\mathrm{CH}), 131.9(\mathrm{CH}), 134.0,135.9,138.8$ $\left(\mathrm{CNO}_{2}\right), 148.3,153.2$ ppm; MS m/z (I $\left.\mathrm{I}_{\mathrm{rel}}, \%\right): 444\left[\mathrm{M}^{+}\right](10), 428[\mathrm{M}-\mathrm{O}]^{+}(5), 360\left[\mathrm{M}-\mathrm{CH}_{2} \mathrm{Cl}_{2}\right](5), 96$ (100); HRMS (ESI ${ }^{+}$) $m / z$ calcd for $\mathrm{C}_{21} \mathrm{H}_{14} \mathrm{~N}_{2} \mathrm{O}_{3} \mathrm{Cl}_{2} \mathrm{SNa}[\mathrm{M}+\mathrm{Na}]^{+}$: 467.0000; found: 466.9998 .

2-[(4-Chlorophenyl)sulfinyl]-4-(dichloromethyl)-3-nitrobenzo[h]quinoline (37b). Same procedure as for 37a, but starting from $36 \mathrm{~b}(0.450 \mathrm{~g}, 1.0 \mathrm{mmol})$. Yield $0.424 \mathrm{~g}(91 \%)$, yellow solid, m.p. $166-168{ }^{\circ} \mathrm{C}$. IR (ATR) $v_{\max }=3082,1546,1342,1092,828,750 \mathrm{~cm}^{-1} .{ }^{1} \mathrm{H}$ NMR $\left(400 \mathrm{MHz}, \mathrm{CDCl}_{3}\right) \delta=7.20\left(1 \mathrm{H}, \mathrm{s}, \mathrm{CHCl}_{2}\right), 7.50$ $(2 \mathrm{H}, \mathrm{d}, J=8.6 \mathrm{~Hz}, \mathrm{H} \mathrm{Ar}), 7.84-7.91(2 \mathrm{H}, \mathrm{m}, \mathrm{H} \mathrm{Ar}), 7.95-8.03(3 \mathrm{H}, \mathrm{m}, \mathrm{H} \mathrm{Ar}), 8.12(1 \mathrm{H}, \mathrm{d}, J=9.4 \mathrm{~Hz}, \mathrm{H}$ Ar), $8.71(1 \mathrm{H}, \mathrm{d}, J=9.4 \mathrm{~Hz}, \mathrm{H} \mathrm{Ar}), 9.28-9.33(1 \mathrm{H}, \mathrm{m}, \mathrm{H} \mathrm{Ar}) \mathrm{ppm} ;{ }^{13} \mathrm{C} \mathrm{NMR}\left(100 \mathrm{MHz}, \mathrm{CDCl}_{3}\right) \delta=62.4$ $\left(\mathrm{CHCl}_{2}\right), 122.0(\mathrm{CH}), 123.3,125.8(\mathrm{CH}), 127.0(2 \times \mathrm{CH}), 128.2(\mathrm{CH}), 128.8(\mathrm{CH}), 129.7(2 \times \mathrm{CH}), 130.5$, $131.0(\mathrm{CH}), 132.0(\mathrm{CH}), 134.0,136.0,138.0\left(\mathrm{CNO}_{2}\right), 138.3,141.1,148.2,153.4(\mathrm{NCS}) \mathrm{ppm} ; \mathrm{MS} m / z\left(I_{\text {rel }}, \%\right)$ : $464\left[\mathrm{M}^{+}\right](2), 418\left[\mathrm{M}-\mathrm{NO}_{2}\right]^{+}(2), 365\left[\mathrm{M}-\mathrm{O}-\mathrm{CHCl}_{2}\right]^{+}(38), 335\left[\mathrm{M}-\mathrm{CHCl}_{2}-\mathrm{NO}_{2}\right]^{+}(12), 111[\mathrm{PhCl}]^{+}(50)$, 100 (100); HRMS (ESI ${ }^{+}$) $\mathrm{m} / z$ calcd for $\mathrm{C}_{20} \mathrm{H}_{11} \mathrm{~N}_{2} \mathrm{O}_{3} \mathrm{Cl}_{3} \mathrm{SNa}[\mathrm{M}+\mathrm{Na}]^{+}$: 486.9454; found: 486.9454 .

Synthesis of 4-(Dichloromethyl)-3-nitro-2-(pyrrolidin-1-yl)benzo[h]quinoline (38). To a solution of quinoline $37 \mathrm{a}(0.445 \mathrm{~g}, 1.0 \mathrm{mmol})$ in toluene $(10 \mathrm{~mL})$, pyrrolidine $(0.107 \mathrm{~g}, 1.5 \mathrm{mmol})$ was added and the mixture heated to $100{ }^{\circ} \mathrm{C}$ for $3 \mathrm{~h}$. After cooling, the crude product was purified by column chromatography using petroleum ether - ethyl acetate (10: 1). Yield $0.290 \mathrm{~g} \mathrm{(77 \% ),} \mathrm{red} \mathrm{solid,} \mathrm{m.p.} 169-170{ }^{\circ} \mathrm{C}$. IR $(\mathrm{ATR}) v_{\max }=2884,1584,1506,1359,821,734 \mathrm{~cm}^{-1} .{ }^{1} \mathrm{H}$ NMR $\left(400 \mathrm{MHz}, \mathrm{CDCl}_{3}\right) \delta=1.99-2.09(4 \mathrm{H}, \mathrm{m}$, $\left.\mathrm{NCCH}_{2}\right), 3.61-3.71\left(4 \mathrm{H}, \mathrm{m}, \mathrm{NCH}_{2}\right), 7.07\left(1 \mathrm{H}, \mathrm{s}, \mathrm{CHCl}_{2}\right), 7.61-7.71(3 \mathrm{H}, \mathrm{m}, \mathrm{H} \mathrm{Ar}), 7.85(1 \mathrm{H}, \mathrm{dd}, J=7.6$ $\mathrm{Hz}, J=1.3 \mathrm{~Hz}, \mathrm{H} \mathrm{Ar}), 8.50(1 \mathrm{H}, \mathrm{d}, J=9.3 \mathrm{~Hz}, \mathrm{H} \mathrm{Ar}), 9.03\left(1 \mathrm{H}, \mathrm{d}, J=7.9 \mathrm{~Hz}, \mathrm{H}\right.$ Ar) ppm; ${ }^{13} \mathrm{C}$ NMR $(100$ $\left.\mathrm{MHz}_{2} \mathrm{CDCl}_{3}\right) \delta=25.5\left(2 \times \mathrm{NCCH}_{2}\right), 48.1\left(2 \times \mathrm{NCH}_{2}\right), 64.0\left(\mathrm{CHCl}_{2}\right), 113.9,122.6(\mathrm{CH}), 124.1(\mathrm{CH}), 125.5$ $(\mathrm{CH}), 126.6(\mathrm{CH}), 127.6(\mathrm{CH}), 129.2(\mathrm{CH}), 130.2,130.4\left(\mathrm{CNO}_{2}\right), 134.3,135.6,145.5,147.5 \mathrm{ppm}$; MS m/z

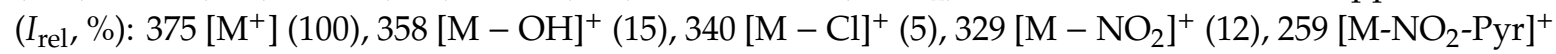
(30); HRMS (ESI ${ }^{+}$) m/z calcd for $\mathrm{C}_{18} \mathrm{H}_{15} \mathrm{~N}_{3} \mathrm{O}_{2} \mathrm{Cl}_{2} \mathrm{Na}[\mathrm{M}+\mathrm{Na}]^{+}$: 398.0439; found: 398.0440 .

Synthesis of 2-ethoxy-2-oxoethyl 4,5-dichloro-1,2-thiazole-3-carboxylate (41). To a solution of isothiazole 40 $(0.198 \mathrm{~g}, 1.0 \mathrm{mmol})$ and ethyl bromoacetate $(0.501 \mathrm{~g}, 3.0 \mathrm{mmol})$ in EtOH $(10 \mathrm{~mL})$, sodium ethanolate $(0.204 \mathrm{~g}, 3.0 \mathrm{mmol})$ was added at r.t. The mixture was heated to reflux for $3 \mathrm{~d}$. The product was extracted with chloroform $(3 \times 10 \mathrm{~mL})$ and purified through a short column chromatography using petroleum ether - ethyl acetate $(10: 1)$. Yield $0.165 \mathrm{~g}(58 \%)$, yellowish solid, m.p. $31-33^{\circ} \mathrm{C} . \mathrm{IR}(\mathrm{KBr})$ $v_{\max }=2983,1743,1427,1193,1038,947 \mathrm{~cm}^{-1} .{ }^{1} \mathrm{H}$ NMR $\left(200 \mathrm{MHz}, \mathrm{CDCl}_{3}\right) \delta=1.31(3 \mathrm{H}, \mathrm{t}, J=7.1 \mathrm{~Hz}$, $\left.\mathrm{OCH}_{2} \underline{\mathrm{CH}}_{3}\right), 4.28\left(2 \mathrm{H}, \mathrm{q}, J=7.1 \mathrm{~Hz}, \mathrm{OCH}_{2} \mathrm{CH}_{3}\right), 4.91\left(2 \mathrm{H}, \mathrm{s}, \mathrm{OCH}_{2} \mathrm{C}=\mathrm{O}\right) \mathrm{ppm} ;{ }^{13} \mathrm{C} \mathrm{NMR}(50 \mathrm{MHz}$, $\left.\mathrm{CDCl}_{3}\right) \delta=14.0\left(\mathrm{CH}_{3}\right), 61.7\left(\mathrm{OCH}_{2} \mathrm{CH}_{3}, \underline{\mathrm{OC}}_{2} \mathrm{C}=\mathrm{O}\right), 126.1(\mathrm{CCl}), 150.8(\mathrm{SCCl}), 153.1(\mathrm{C}=\mathrm{N}), 158.2,166.7$; MS $m / z\left(I_{\mathrm{rel}}, \%\right): 283\left[\mathrm{M}^{+}\right](18), 238[\mathrm{M}-\overline{\mathrm{OEt}}]^{+}(15), 210\left[\mathrm{M}-\mathrm{CO}_{2} \mathrm{Et}\right]^{+}(12), 180\left[\mathrm{M}-\mathrm{OCH}_{2} \mathrm{CO}_{2} \mathrm{Et}\right]^{+}$ (100); HRMS (ESI ${ }^{+}$) m/z calcd for $\mathrm{C}_{8} \mathrm{H}_{7} \mathrm{NO}_{4} \mathrm{Cl}_{2} \mathrm{SNa}[\mathrm{M}+\mathrm{Na}]^{+}: 305.9371$; found: 305.9370 . 
Synthesis of 2,2,2-Trifluoroethyl 4,5-dichloro-1,2-thiazole-3-carboxylate (43). A solution of isothiazole 42 $(0.217 \mathrm{~g}, 1.0 \mathrm{mmol})$ and 2,2,2-trifluoroethanol $(0.300 \mathrm{~g}, 4.0 \mathrm{mmol})$ in $20 \mathrm{~mL}$ dry THF was refluxed for $4 \mathrm{~d}$. After cooling to r.t., the solution was concentrated, diluted with water $(20 \mathrm{~mL})$, extracted with hexane $(3 \times 20 \mathrm{~mL})$, and washed with water $(2 \times 20 \mathrm{~mL})$. Subsequently, the product was purified by column chromatography (hexane). Yield $0.179 \mathrm{~g}(64 \%)$, white solid, m.p. $51-52{ }^{\circ} \mathrm{C}$. IR $(\mathrm{KBr}) v_{\max }=$ $\mathrm{cm}^{-1}: 1741,1430,1358,1167,1091,745 .{ }^{1} \mathrm{H} \mathrm{NMR}\left(200 \mathrm{MHz} \mathrm{CDCl}_{3}\right) \delta=4.61\left(2 \mathrm{H}, \mathrm{q}, J=8.2 \mathrm{~Hz}, \mathrm{OCH}_{2}\right)$; ${ }^{13} \mathrm{C} \mathrm{NMR}\left(50 \mathrm{MHz}, \mathrm{CDCl}_{3}\right) \delta=61.3\left(\mathrm{JC}_{\mathrm{C}, \mathrm{F}}=37.1 \mathrm{~Hz}, \mathrm{CH}_{2}\right), 122.6\left(\mathrm{~J}_{\mathrm{C}, \mathrm{F}}=276.0 \mathrm{~Hz}, \mathrm{CF}_{3}\right), 126.3(\mathrm{CCl})$, $151.3(\mathrm{C}=\mathrm{N}), 152.3(\mathrm{SCCl}), 157.3(\mathrm{C}=\mathrm{O}) \mathrm{ppm} ; \mathrm{MS} \mathrm{m} / \mathrm{z}\left(\mathrm{I}_{\mathrm{rel}}, \%\right): 279$ [M $\left.^{+}\right](15), 244[\mathrm{M}-\mathrm{Cl}]^{+}(2), 197$ $\left[\mathrm{M}-\mathrm{CH}_{2} \mathrm{CF}_{3}+\mathrm{H}\right]^{+}(12), 180\left[\mathrm{M}-\mathrm{OCH}_{2} \mathrm{CF}_{3}\right]^{+}(100), 153\left[\mathrm{M}-\mathrm{CO}_{2} \mathrm{CH}_{2} \mathrm{CF}_{3}+\mathrm{H}\right]^{+}(40) ; \mathrm{HRMS}\left(\mathrm{ESI}^{+}\right) \mathrm{m} / \mathrm{z}$ calcd for $\mathrm{C}_{6} \mathrm{H}_{2} \mathrm{NO}_{2} \mathrm{Cl}_{2} \mathrm{SF}_{3} \mathrm{Na}[\mathrm{M}+\mathrm{Na}]^{+}$: 301.9033; found: 301.9035 .

Synthesis of 4,5-Dichloro-N'-phenyl-1,2-thiazole-3-carbohydrazide (44a) (General method). To a suspension of phenylhydrazine $(0.324 \mathrm{~g}, 3.0 \mathrm{mmol})$ in dry THF $(10 \mathrm{~mL})$, the isothiazole $42(0.216 \mathrm{~g}, 1.0 \mathrm{mmol})$ was added at r.t. The mixture was stirred for $1 \mathrm{~d}$. After removal of the solvent, the residue was treated with cold $\mathrm{HCl}(10 \%)$ and the resulting precipitate filtered off. Subsequently, it was washed with cold water $(3 \times 5 \mathrm{~mL})$ and $\mathrm{Et}_{2} \mathrm{O}(2 \times 3 \mathrm{~mL})$. The product was dried in vacuo. Yield $0.216 \mathrm{~g}(75 \%)$, light brown solid, m.p. $145-147^{\circ} \mathrm{C}$. IR $(\mathrm{KBr}) v_{\max }=3254,1678,1605,1351,884,746 \mathrm{~cm}^{-1} .{ }^{1} \mathrm{H}$ NMR $\left(200 \mathrm{MHz}, \mathrm{CDCl}_{3}\right) \delta$ $=6.88-6.96(3 \mathrm{H}, \mathrm{m}, \mathrm{H} \mathrm{Ph}), 7.20-7.26(3 \mathrm{H}, \mathrm{m}, \mathrm{NH}, 2 \mathrm{H} \mathrm{Ph}), 8.81(1 \mathrm{H}, \mathrm{s}, \mathrm{NH}) \mathrm{ppm} ;{ }^{13} \mathrm{C} \mathrm{NMR}(50 \mathrm{MHz}$, $\left.\mathrm{CDCl}_{3}\right) \delta=113.8(2 \times \mathrm{CH}), 121.6(\mathrm{CH}), 125.3(\mathrm{CCl}), 129.2(2 \times \mathrm{CH}), 147.3,150.9(\mathrm{SCCl}), 155.3,158.9$

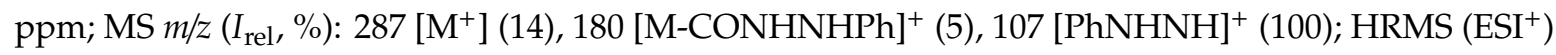
$m / z$ calcd for $\mathrm{C}_{10} \mathrm{H}_{7} \mathrm{~N}_{3} \mathrm{OCl}_{2} \mathrm{SNa}[\mathrm{M}+\mathrm{Na}]^{+}$: 309.9585 ; found: 309.9585 .

4,5-Dichloro-N'-[3-(trifluoromethyl)phenyl]-1,2-thiazole-3-carbohydrazide (44b). Same procedure as for 44a, but using 3-trifluoromethylphenylhydrazine $(0.370 \mathrm{~g}, 2.1 \mathrm{mmol})$ and stirring the mixture at reflux for 4 h. Yield $0.338 \mathrm{~g}$ (95\%), yellowish solid, m.p. 152-153 ${ }^{\circ} \mathrm{C}$. IR (KBr) $v_{\max }=3300,1691,1497,1340,1161$, $798 \mathrm{~cm}^{-1} .{ }^{1} \mathrm{H}$ NMR (200 MHz, DMSO-d 6 ) $\delta=7.03-7.09$ (3H, m, H Ar), 7.34-7.49 (1H, m, H Ar), 8.57 $(1 \mathrm{H}, \mathrm{s}, \mathrm{NH}), 10.79(1 \mathrm{H}, \mathrm{s}, \mathrm{NH}) \mathrm{ppm} ;{ }^{13} \mathrm{C} \mathrm{NMR}\left(50 \mathrm{MHz}, \mathrm{DMSO}-\mathrm{d}_{6}\right) \delta=108.2\left(J_{\mathrm{C}, \mathrm{F}}=3.9 \mathrm{~Hz}, \mathrm{CH}\right), 115.2$ $\left(J_{\mathrm{C}, \mathrm{F}}=4.0 \mathrm{~Hz}, \mathrm{CH}\right), 116.1(\mathrm{CH}), 124.6\left(J_{\mathrm{C}, \mathrm{F}}=271.5 \mathrm{~Hz}, \mathrm{CF}_{3}\right), 123.0(\mathrm{CCl}), 129.7\left(J_{\mathrm{C}, \mathrm{F}}=31.3 \mathrm{~Hz}, \mathrm{CCF}_{3}\right)$,

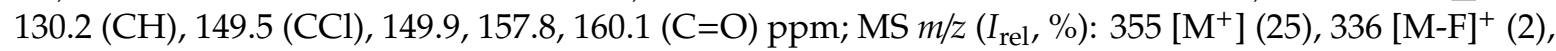
180 [M-CF $\left._{3}-\mathrm{Ph}-\mathrm{NHNH}\right]^{+}(60), 175$ [CF $\left._{3}-\mathrm{Ph}-\mathrm{NHNH}\right]^{+}(100), 152$ [M-CONHNH-Ph-CF $\left._{3}\right]^{+}$(10); HRMS $\left(\mathrm{ESI}^{+}\right) \mathrm{m} / \mathrm{z}$ calcd for $\mathrm{C}_{11} \mathrm{H}_{6} \mathrm{~N}_{3} \mathrm{OCl}_{2} \mathrm{SF}_{3} \mathrm{Na}[\mathrm{M}+\mathrm{Na}]^{+}: 377.9458$; found: 377.9459 .

4,5-Dichloro-N-(4-cyano-2,5-difluorophenyl)-1,2-thiazole-3-carboxamide (44c). Same procedure as for 44a, but using 4-cyano-2,5-difluoro-aniline $(0.354 \mathrm{~g}, 2.3 \mathrm{mmol})$ and stirring the mixture at reflux for $6 \mathrm{~h}$. Yield $0.227 \mathrm{~g}(68 \%)$, white solid, m.p. $164-166^{\circ} \mathrm{C}$. IR (KBr) $v_{\max }=3361,2237$ (CN), 1715, 1528, 1190, $673 \mathrm{~cm}^{-1} .{ }^{1} \mathrm{H}$ NMR $\left(200 \mathrm{MHz}, \mathrm{CDCl}_{3}\right) \delta=7.41(1 \mathrm{H}, \mathrm{dd}, J=9.8 \mathrm{~Hz}, J=5.4 \mathrm{~Hz}, \mathrm{H} \mathrm{Ar}), 8.57(1 \mathrm{H}, \mathrm{dd}$, $J=10.6 \mathrm{~Hz}, J=6.2 \mathrm{~Hz}, \mathrm{H} \mathrm{Ar}), 9.49(1 \mathrm{H}, \mathrm{s}, \mathrm{NH}) \mathrm{ppm} ;{ }^{13} \mathrm{C} \mathrm{NMR}\left(50 \mathrm{MHz}, \mathrm{CDCl}_{3}\right) \delta=95.6\left(J_{\mathrm{C}, \mathrm{F}}=\right.$ $18.8 \mathrm{~Hz}, 9.6 \mathrm{~Hz}, \underline{\mathrm{C}}(\mathrm{CN})), 108.6\left(J_{\mathrm{C}, \mathrm{F}}=28.7 \mathrm{~Hz}, 1.5 \mathrm{~Hz}, \mathrm{CH}\right), 112.9\left(J_{\mathrm{C}, \mathrm{F}}=2.3 \mathrm{~Hz}, \mathrm{C}(\underline{\mathrm{CN}})\right), 118.3\left(J_{\mathrm{C}, \mathrm{F}}=\right.$ $24.5 \mathrm{~Hz}, 2.3 \mathrm{~Hz}, \mathrm{CH}), 125.7(\mathrm{CCl}), 131.7$ (JC,F $=11.9 \mathrm{~Hz}, \mathrm{NC} \mathrm{Ph}), 147.5\left(J_{\mathrm{C}, \mathrm{F}}=244.0 \mathrm{~Hz}, 2.8 \mathrm{~Hz}, \mathrm{CF}\right)$, $152.1(\mathrm{CCl}), 154.6,156.3(\mathrm{C}=\mathrm{O}), 160.1\left(J_{\mathrm{C}, \mathrm{F}}=255.3 \mathrm{~Hz}, 2.3 \mathrm{~Hz}, \mathrm{CF}\right) \mathrm{ppm} ; \mathrm{MS} m / z\left(I_{\mathrm{rel}}, \%\right): 333$ [M $\left.{ }^{+}\right]$ (22), 180 [M-NH-Ph-F $\left.{ }_{2}-(\mathrm{CN})\right]^{+}$(100), 152 [M-CONH-Ph-F $\left.2_{2}(\mathrm{CN})\right]^{+}(15) ; \mathrm{HRMS}\left(\mathrm{ESI}^{+}\right) \mathrm{m} / z$ calcd for $\mathrm{C}_{11} \mathrm{H}_{3} \mathrm{~N}_{3} \mathrm{OCl}_{2} \mathrm{SF}_{2} \mathrm{Na}[\mathrm{M}+\mathrm{Na}]^{+}$: 355.9240; found: 355.9241 .

4,5-Dichloro-N-[2-(1H-indol-3-yl)ethyl]-1,2-thiazole-3-carboxamide (44d). Same procedure as for 44a, but using tryptamine $(0.352 \mathrm{~g}, 2.2 \mathrm{mmol})$ and triethylamine $(0.223 \mathrm{~g}, 2.1 \mathrm{mmol})$. Yield $0.265 \mathrm{~g}(78 \%)$, white solid, m.p. $183-185^{\circ} \mathrm{C}$. IR $(\mathrm{KBr}) v_{\max }=3302,1650,1537,1349,946,738 \mathrm{~cm}^{-1}$. ${ }^{1} \mathrm{H} \mathrm{NMR}(200 \mathrm{MHz}$, $\left.\mathrm{CDCl}_{3}\right) \delta=3.08\left(2 \mathrm{H}, \mathrm{t}, J=8.1 \mathrm{~Hz}\right.$, Ind- $\left.\mathrm{CH}_{2}\right), 3.72-3.82\left(2 \mathrm{H}, \mathrm{m}, \mathrm{NCH}_{2}\right), 7.07(1 \mathrm{H}, \mathrm{s}, \mathrm{NHC}=\mathrm{O}), 7.11-7.25$ $(3 \mathrm{H}, \mathrm{m}, 2 \mathrm{H} \mathrm{Ar}, \mathrm{NCH}), 7.37(1 \mathrm{H}, \mathrm{d}, J=7.7 \mathrm{~Hz}, \mathrm{H} \mathrm{Ar}), 7.63(1 \mathrm{H}, \mathrm{d}, J=7.7 \mathrm{~Hz}, \mathrm{H} \mathrm{Ar}), 8.15(1 \mathrm{H}, \mathrm{s}, \mathrm{NH})$ ppm; ${ }^{13} \mathrm{C} \mathrm{NMR}\left(50 \mathrm{MHz}, \mathrm{CDCl}_{3}\right) \delta=25.3\left(\mathrm{CH}_{2}\right), 39.6\left(\mathrm{CH}_{2}\right), 111.2(\mathrm{CH}), 112.6,118.7(\mathrm{CH}), 119.5(\mathrm{CH})$, $122.1(\mathrm{CH}, \mathrm{CCl}), 122.2(\mathrm{CH}), 127.2,136.4,150.4(\mathrm{CCl}), 156.7,159.0(\mathrm{C}=\mathrm{O}) \mathrm{ppm}$; MS m/z $\left(I_{\mathrm{rel}}, \%\right): 339$ $\left[\mathrm{M}^{+}\right]$(4), $180\left[\mathrm{M}-\mathrm{NHCH}_{2} \text {-ind }\right]^{+}$(5), 143 [ind-CHCH$]^{+}$(100), 130 [ind-CH$]^{+}(91) ; \mathrm{HRMS}\left(\mathrm{ESI}^{+}\right) \mathrm{m} / z$ calcd for $\mathrm{C}_{14} \mathrm{H}_{11} \mathrm{~N}_{3} \mathrm{OCl}_{2} \mathrm{SNa}[\mathrm{M}+\mathrm{Na}]^{+}$: 361.9900; found: 361.9898 . 
4,5-Dichloro-N-[(6-chloropyridin-3-yl)methyl]-N-methyl-1,2-thiazole-3-carboxamide (44e). Same proce-dure as for 44a, but using isothiazole 42 and 1-(6-chloropyridin-3-yl)-N-methylmethanamine (0.313 g, 2.0 $\mathrm{mmol})$ at $0{ }^{\circ} \mathrm{C}$ and then stirring at r.t. for $5 \mathrm{~h}$. Yield $0.290 \mathrm{~g}(86 \%)$.

Alternative synthesis of thiazole 44e: To a suspension of 4,5-dichloro- $N$ '-phenyl-1,2-thiazole-3-carbohydrazide (44a) (0.288 g, $1.0 \mathrm{mmol})$ in DMSO (10 $\mathrm{mL}), 1$-(6-chloropyridin-3-yl)- $N$-methylmethanamine $(0.313 \mathrm{~g}, 2.0 \mathrm{mmol})$ was added. The mixture was heated to $100{ }^{\circ} \mathrm{C}$ for $8 \mathrm{~h}$. After cooling to r.t., water $(50 \mathrm{~mL})$ was added and the mixture extracted with chloroform $(3 \times 15 \mathrm{~mL})$. Subsequently, the product was purified by column chromatography using petroleum ether-ethyl acetate (3: 1). The product was dried in vacuo. Yield $0.232 \mathrm{~g} \mathrm{(69 \% ),} \mathrm{a} \mathrm{mixture}$ of two rotamers in relation 10: 6, yellowish solid, m.p. $43-45^{\circ} \mathrm{C}$. IR (KBr) $v_{\max }=2934,1651,1460$, $1349,1106,834 \mathrm{~cm}^{-1} .{ }^{1} \mathrm{H} \mathrm{NMR}\left(600 \mathrm{MHz} \mathrm{CDCl}_{3}\right)$ for major isomer $\delta=2.90\left(3 \mathrm{H}, \mathrm{s}, \mathrm{NCH}_{3}\right), 4.73(2 \mathrm{H}, \mathrm{s}$, $\left.\mathrm{NCH}_{2}\right), 7.34(1 \mathrm{H}, \mathrm{d}, J=8.2 \mathrm{~Hz}, \mathrm{H}$ pyr $), 7.71(1 \mathrm{H}, \mathrm{dd}, J=2.5 \mathrm{~Hz}, J=8.3 \mathrm{~Hz}, \mathrm{H}$ pyr $), 8.37(1 \mathrm{H}, \mathrm{d}, J=2.3$ $\mathrm{Hz}, \mathrm{H}$ pyr) ppm; minor isomer $\delta=3.02\left(1.8 \mathrm{H}, \mathrm{s}, \mathrm{NCH}_{3}\right), 4.48\left(1.2 \mathrm{H}, \mathrm{s}, \mathrm{NCH}_{2}\right), 7.34(0.6 \mathrm{H}, \mathrm{d}, J=8.2$ $\mathrm{Hz}, \mathrm{H}$ pyr), $7.72(0.6 \mathrm{H}, \mathrm{dd}, J=2.5 \mathrm{~Hz}, J=8.3 \mathrm{~Hz}, \mathrm{H}$ pyr $), 8.28(0.6 \mathrm{H}, \mathrm{d}, J=2.3 \mathrm{~Hz}, \mathrm{H}$ pyr $)$ ppm; ${ }^{13} \mathrm{C}$ NMR $\left(150 \mathrm{MHz}, \mathrm{CDCl}_{3}\right)$ for major isomer $\delta=35.7\left(\mathrm{NCH}_{3}\right), 47.8\left(\mathrm{NCH}_{2}\right), 122.5(\mathrm{CCl}), 124.6(\mathrm{CH}), 130.7$ $\left(\mathrm{CH}_{2} \mathrm{C}\right), 138.8(\mathrm{CH}), 149.2(\mathrm{CH}), 149.6(\mathrm{CCl}), 151.2(\mathrm{NCCl}), 159.6,162.8(\mathrm{C}=\mathrm{O})$ ppm; minor isomer $\delta=$ $32.8\left(\mathrm{NCH}_{3}\right), 51.2\left(\mathrm{NCH}_{2}\right), 123.2(\mathrm{CCl}), 124.5(\mathrm{CH}), 130.3\left(\mathrm{CH}_{2} \mathrm{C}\right), 138.1(\mathrm{CH}), 148.9(\mathrm{CH}), 149.8(\mathrm{CCl})$, $151.5(\mathrm{NCCl}), 159.5,162.4(\mathrm{C}=\mathrm{O}) \mathrm{ppm} ;{ }^{15} \mathrm{~N} \mathrm{NMR}\left(43.4 \mathrm{MHz}, \mathrm{CDCl}_{3}\right.$, doped with nitromethane $(0.0$ ppm)) $\delta=-267.5$ (NMe, minor isomer), -266.3 (NMe, major isomer), -75.7 (NCCl, major isomer), -75.5

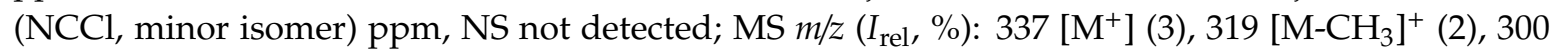
$[\mathrm{M}-\mathrm{Cl}]^{+}(6), 180$ [M-CH$_{3}-\mathrm{N}-\mathrm{CH}_{2}$-isothiazole $]^{+}$(14), 155 [isothiazole-N-CH$\left._{2}-\mathrm{CH}_{3}\right]^{+}(100) ; \mathrm{HRMS}_{(\mathrm{ESI}}^{+}$) $m / z$ calcd for $\mathrm{C}_{11} \mathrm{H}_{8} \mathrm{~N}_{3} \mathrm{OCl}_{3} \mathrm{SNa}[\mathrm{M}+\mathrm{Na}]^{+}: 357.9351$; found: 357.9352.

4-Chloro-5-(morpholin-4-yl)-N'-phenyl-1,2-thiazole-3-carbohydrazide (45). Following the alternative procedure for azole $44 \mathrm{e}$ using morpholine $(0.348 \mathrm{~g}, 4.0 \mathrm{mmol})$. The mixture was stirred at $90-95^{\circ} \mathrm{C}$ for 4 h. Yield $0.264 \mathrm{~g}(78 \%)$, yellowish solid, m.p. $163-164{ }^{\circ} \mathrm{C}$. IR (ATR) $v_{\max }=3294,1695,1494,1403,1115$, $688 \mathrm{~cm}^{-1}$. ${ }^{1} \mathrm{H}$ NMR $\left(400 \mathrm{MHz} \mathrm{CDCl}_{3}\right) \delta=3.32-3.41\left(4 \mathrm{H}, \mathrm{m}, \mathrm{NCH}_{2}\right), 3.82-3.91\left(4 \mathrm{H}, \mathrm{m}, \mathrm{OCH}_{2}\right), 6.24$ (1H, s, Ph-NH), 6.88-6.95 (3H, m, H Ph), 7.21-7.26 (2H, m, H Ph), 8.78 (1H, s, CONH) ppm; ${ }^{13} \mathrm{C} \mathrm{NMR}$ $\left(100 \mathrm{MHz}_{,} \mathrm{CDCl}_{3}\right) \delta=50.8\left(2 \times \mathrm{NCH}_{2}\right), 66.0\left(2 \times \mathrm{OCH}_{2}\right), 108.9,113.8(2 \times \mathrm{CH}), 121.4(\mathrm{CH}), 129.2(2 \times$ $\mathrm{CH}), 147.7,156.0(\mathrm{NCC}=\mathrm{O}), 160.1(\mathrm{C}=\mathrm{O}), 173.1$ (morph-C $)$ ppm; MS m/z (I $\left.\mathrm{rel}_{1} \%\right): 338\left[\mathrm{M}^{+}\right](75), 303$ $[\mathrm{M}-\mathrm{Cl}]^{+}$(8), 231 [M-PhNHNH] $^{+}$(35), 197 [M-PhNHNH-Cl+H] $^{+}$(100), 107 [PhNHNH] $^{+}$(60); HRMS $\left(\mathrm{ESI}^{+}\right) \mathrm{m} / \mathrm{z}$ calcd for $\mathrm{C}_{14} \mathrm{H}_{15} \mathrm{~N}_{4} \mathrm{O}_{2} \mathrm{ClSNa}[\mathrm{M}+\mathrm{Na}]^{+}$: 361.0502; found: 361.0502 .

Synthesis of 4-chloro-5-[(4-chlorophenyl)sulfanyl]-N'-phenyl-1,2-thiazole-3-carbohydrazide (46a) (General method). To a solution of isothiazole $44 \mathrm{a}(0.288 \mathrm{~g}, 1.0 \mathrm{mmol})$ in DMSO $(10 \mathrm{~mL})$, 4-chlorothiophenol $(0.173 \mathrm{~g}, 1.2 \mathrm{mmol})$ and sodium ethanolate $(0.082 \mathrm{~g}, 1.2 \mathrm{mmol})$ were added. The mixture was stirred at $110{ }^{\circ} \mathrm{C}$ for $8 \mathrm{~h}$. After concentration at $100{ }^{\circ} \mathrm{C}$, it was poured into $50 \mathrm{~mL}$ diluted $\mathrm{HCl}(5 \%)$ and extracted with chloroform $(3 \times 20 \mathrm{~mL})$. The combined organic phases were washed with water, dried over calcium chloride, and purified by column chromatography using petroleum ether-ethyl acetate (2:1). Subsequently, the dried product was washed with cold methanol $(1 \times 3 \mathrm{~mL})$. Yield $0.266 \mathrm{~g}(67 \%)$, white solid, m.p. $169-170{ }^{\circ} \mathrm{C}$. IR (ATR) $\nu_{\max }=3250,1655,1493,1094,896,695 \mathrm{~cm}^{-1} .{ }^{1} \mathrm{H}$ NMR $(400$ $\left.\mathrm{MHz} \mathrm{CDCl}_{3}\right) \delta=5.11(1 \mathrm{H}, \mathrm{s}$ br, Ph-NH), 6.88-6.95 (3H, m, H Ph), 7.20-7.26 (2H, m, H Ph), 7.43 (2H, $\mathrm{d}, J=8.5 \mathrm{~Hz}, \mathrm{H} \mathrm{Ar}), 7.53(2 \mathrm{H}, \mathrm{d}, J=8.5 \mathrm{~Hz}, \mathrm{H} \mathrm{Ar}), 8.7(1 \mathrm{H}, \mathrm{s}, \mathrm{CONH}) \mathrm{ppm} ;{ }^{13} \mathrm{C} \mathrm{NMR}(100 \mathrm{MHz}$, $\left.\mathrm{CDCl}_{3}\right) \delta=113.8(2 \times \mathrm{CH}), 121.6(\mathrm{CH}), 123.3,128.2(\mathrm{CCl}), 129.2(2 \times \mathrm{CH}), 130.5(2 \times \mathrm{CH}), 134.7(2 \times$ $\mathrm{CH}), 136.7$ (CS-Thiaz), 147.5, 155.5 (CC=O), 159.3, $160.9(\mathrm{C}=\mathrm{O}) \mathrm{ppm} ; \mathrm{MS}$ m/z $\left(\mathrm{I}_{\mathrm{rel}}, \%\right): 395\left[\mathrm{M}^{+}\right](20), 288$

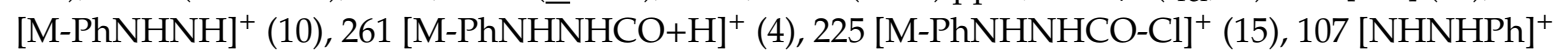
(100); HRMS (ESI ${ }^{+}$) $\mathrm{m} / z$ calcd for $\mathrm{C}_{16} \mathrm{H}_{11} \mathrm{~N}_{3} \mathrm{OCl}_{2} \mathrm{~S}_{2} \mathrm{Na}[\mathrm{M}+\mathrm{Na}]^{+}$: 417.9618; found: 417.9617.

4-Chloro-5-[(4-chlorophenyl)sulfanyl]-N'-[3-(trifluoromethyl)phenyl]-1,2-thiazole-3-carbohydrazide (46b). Same procedure as for $46 \mathrm{a}$, but using $44 \mathrm{~b}(0.356 \mathrm{~g}, 1.0 \mathrm{mmol})$. The product was purified using petroleum ether-ethyl acetate (10: 1). Yield $0.325 \mathrm{~g}(70 \%)$, white solid, m.p. $144-145^{\circ} \mathrm{C}$. IR (ATR) $v_{\max }$ 
$=3246,1662,1476,1338,1123,694 \mathrm{~cm}^{-1} .{ }^{1} \mathrm{H}$ NMR $\left(400 \mathrm{MHz}, \mathrm{CDCl}_{3}\right) \delta=6.39(1 \mathrm{H}, \mathrm{s} \mathrm{br}, \mathrm{NH}), 7.05(1 \mathrm{H}$, $\mathrm{dd}, J=8.0 \mathrm{~Hz}, J=2.1 \mathrm{~Hz}, \mathrm{H} \mathrm{Ar}), 7.11(1 \mathrm{H}, \mathrm{s}, \mathrm{H} \mathrm{Ar}), 7.15(1 \mathrm{H}, \mathrm{d}, J=7.9 \mathrm{~Hz}, \mathrm{H} \mathrm{Ar}), 7.32(1 \mathrm{H}, \mathrm{t}, J=8.0 \mathrm{~Hz}$, $\mathrm{H} \mathrm{Ar}), 7.44(2 \mathrm{H}, \mathrm{d}, J=8.6 \mathrm{~Hz}, \mathrm{H} \mathrm{Ar}), 7.54(2 \mathrm{H}, \mathrm{d}, J=8.6 \mathrm{~Hz}, \mathrm{H} \mathrm{Ar}), 8.77(1 \mathrm{H}, \mathrm{s}, \mathrm{NH}) .{ }^{13} \mathrm{C}(100 \mathrm{MHz}$, $\left.\mathrm{CDCl}_{3}\right) \delta=110.2\left(\mathrm{~J}_{\mathrm{C}, \mathrm{F}}=4.1 \mathrm{~Hz}, \mathrm{CH}\right), 116.8(\mathrm{CH}), 118.1\left(\mathrm{~J}_{\mathrm{C}, \mathrm{F}}=3.9 \mathrm{~Hz}, \mathrm{CH}\right), 123.9\left(\mathrm{~J}_{\mathrm{C}, \mathrm{F}}=272.5 \mathrm{~Hz}, \mathrm{CF}_{3}\right)$, $123.1(\mathrm{CCl}), 128.0(\mathrm{CCl}), 129.7(\mathrm{CH}), 130.9(2 \times \mathrm{CH}), 131.6\left(\mathrm{~J}_{\mathrm{C}, \mathrm{F}}=32.2 \mathrm{~Hz}, \mathrm{CCF}_{3}\right), 134.8(2 \times \mathrm{CH}), 136.9$

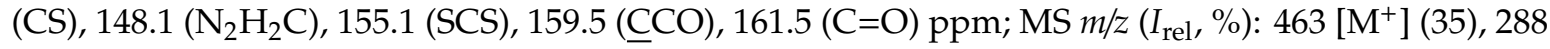
$\left[\mathrm{M}-\mathrm{N}_{2} \mathrm{H}_{2} \mathrm{PhCF}_{3}\right]^{+}$(93), $225\left[\mathrm{M}-\mathrm{CON}_{2} \mathrm{H}_{2} \mathrm{PhCF}_{3}\right]^{+}(80), 145\left[\mathrm{PhCF}_{3}\right]^{+}(100) ; \mathrm{HRMS}\left(\mathrm{ESI}^{+}\right) \mathrm{m} / z$ calcd for $\mathrm{C}_{17} \mathrm{H}_{10} \mathrm{~N}_{3} \mathrm{OCl}_{2} \mathrm{~S}_{2} \mathrm{~F}_{3} \mathrm{Na}[\mathrm{M}+\mathrm{Na}]^{+}:$485.9492; found: 485.9494.

4-Chloro-5-[(4-chlorophenyl)sulfanyl]-N-(4-cyano-2,5-difluorophenyl)-1,2-thiazole-3-carboxamide (46c). Same procedure as for $46 \mathrm{a}$, but using $44 \mathrm{c}(0.334 \mathrm{~g}, 1.0 \mathrm{mmol})$. The product was purified by using petroleum ether-ethyl acetate (10: 1). Yield $0.367 \mathrm{~g}(83 \%)$, white solid, m.p. $152-153{ }^{\circ} \mathrm{C}$. IR (ATR) $v_{\max }=3384$, $2241(\mathrm{CN}), 1703,1473,1196,617 \mathrm{~cm}^{-1} .{ }^{1} \mathrm{H}$ NMR $\left(400 \mathrm{MHz}, \mathrm{CDCl}_{3}\right) \delta=7.24-7.66$ (5H, m, H Ar), 8.57 $\left(1 \mathrm{H}, \mathrm{s}, \mathrm{H}\right.$ Ar), $9.47(1 \mathrm{H}, \mathrm{s}, \mathrm{NH}) \mathrm{ppm} ;{ }^{13} \mathrm{C} \mathrm{NMR}\left(101 \mathrm{MHz}, \mathrm{CDCl}_{3}\right) \delta=95.4\left(J_{\mathrm{C}, \mathrm{F}}=19.8 \mathrm{~Hz}, 11.0 \mathrm{~Hz}, \mathrm{Ar}\right.$ $\underline{\mathrm{CCN}}), 108.6\left(J_{\mathrm{C}, \mathrm{F}}=28.6 \mathrm{~Hz}, \mathrm{CH}\right), 112.9(\mathrm{Ph}-\mathrm{CN}), 118.3\left(J_{\mathrm{C}, \mathrm{F}}=24.2 \mathrm{~Hz}, \mathrm{CH}\right), 123.0(\mathrm{CCl}), 127.6(\mathrm{CCl})$, $130.7(2 \times \mathrm{CH}), 131.9\left(\mathrm{~J}_{\mathrm{C}, \mathrm{F}}=11.7 \mathrm{~Hz}, \mathrm{CNH}\right), 135.0(2 \times \mathrm{CH}), 137.1(\mathrm{SC}), 147.5\left(\mathrm{~J}_{\mathrm{C}, \mathrm{F}}=244.3 \mathrm{~Hz}, \mathrm{CF}\right), 154.9$ (SCS), $156.9(\mathrm{SNC}), 160.2\left(J_{\mathrm{C}, \mathrm{F}}=253.8 \mathrm{~Hz}, \mathrm{CF}\right), 163.0(\mathrm{C}=\mathrm{O}) \mathrm{ppm}$; MS m/z (I $\left.\mathrm{I}_{\mathrm{rel}}, \%\right): 441\left[\mathrm{M}^{+}\right](40), 288$

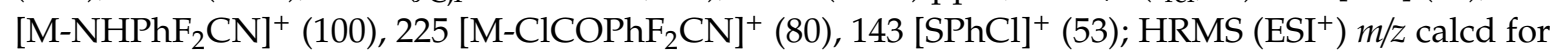
$\mathrm{C}_{17} \mathrm{H}_{7} \mathrm{~N}_{3} \mathrm{OCl}_{2} \mathrm{~S}_{2} \mathrm{~F}_{2} \mathrm{Na}[\mathrm{M}+\mathrm{Na}]^{+}$: 463.9273; found: 463.9271 .

4-Chloro-5-[(4-chlorophenyl)sulfanyl]-N-[2-(1H-indol-3-yl)ethyl]-1,2-thiazole-3-carboxamide (46d). Same procedure as for $46 \mathrm{a}$, but using $44 \mathrm{~d}(0.340 \mathrm{~g}, 1.0 \mathrm{mmol})$. After addition of $\mathrm{HCl}$ the crude product precipitated and was filtered off, washed with diluted $\mathrm{HCl}(3 \mathrm{~mL})$, water $(2 \times 5 \mathrm{~mL})$, cold $\mathrm{MeOH}(3$ $\mathrm{mL}$ ) and dried in vacuo. Yield $0.399 \mathrm{~g}(89 \%)$, white solid, m.p. $176-177^{\circ} \mathrm{C}$. IR (ATR) $v_{\max }=3280,1667$, $1525,1338,821,740 \mathrm{~cm}^{-1} .{ }^{1} \mathrm{H}$ NMR $\left(400 \mathrm{MHz}, \mathrm{DMSO}_{6}\right) \delta=2.88-2.99\left(2 \mathrm{H}, \mathrm{m}, \mathrm{Ind}_{6}-\mathrm{CH}_{2}\right), 3.47-3.58$ $\left(2 \mathrm{H}, \mathrm{m}, \mathrm{NCH}_{2}\right), 6.98(1 \mathrm{H}, \mathrm{t}, J=7.0 \mathrm{~Hz}, \mathrm{H}$ ind $), 7.06(1 \mathrm{H}, \mathrm{t}, J=7.0 \mathrm{~Hz}, \mathrm{H}$ ind $), 7.19(1 \mathrm{H}, \mathrm{s}, \mathrm{H}$ ind $), 7.34$ $(1 \mathrm{H}, \mathrm{d}, J=7.7 \mathrm{~Hz}, \mathrm{H}$ ind $), 7.51-7.73(5 \mathrm{H}, \mathrm{m}, \mathrm{H}$ ind, $4 \mathrm{H} \mathrm{Ar}), 8.84(1 \mathrm{H}, \mathrm{s}, \mathrm{CONH}), 10.82(1 \mathrm{H}, \mathrm{s}, \mathrm{NH}$ ind); ${ }^{13} \mathrm{C}$ NMR $\left(101 \mathrm{MHz}, \mathrm{DMSO}-\mathrm{d}_{6}\right) \delta=25.1$ (ind $\left.-\mathrm{CH}_{2}\right), 39.7\left(\mathrm{NCH}_{2}\right), 111.5,111.6(\mathrm{CH}), 118.4(\mathrm{CH}), 118.5$ (CH), 121.1 (CH), 121.5, $122.8(\mathrm{NCH}), 127.4,128.8,130.6(2 \times \mathrm{CH}), 134.5(2 \times \mathrm{CH}), 135.3(\mathrm{SC}), 136.4$, 158.6 (CCO), 159.4 (SCS), 159.8 (C=O) ppm; MS m/z ( $\left.I_{\text {rel }}, \%\right): 447\left[\mathrm{M}^{+}\right]$(3), 288 [M-NHC ${ }_{2} \mathrm{H}_{4}$-ind] $]^{+}(1)$, $143\left[\mathrm{SPhCl}^{+}(100), 130[\mathrm{IndCH}]^{+}(53) ; \mathrm{HRMS}\left(\mathrm{ESI}^{+}\right) \mathrm{m} / z\right.$ calcd for $\mathrm{C}_{20} \mathrm{H}_{15} \mathrm{~N}_{3} \mathrm{OCl}_{2} \mathrm{~S}_{2} \mathrm{Na}[\mathrm{M}+\mathrm{Na}]^{+}$: 469.9931; found: 469.9931.

2-Ethoxy-2-oxoethyl 4-chloro-5-[(4-chlorophenyl)sulfanyl]-1,2-thiazole-3-carboxylate (46e). Same proce-dure as for 46a, but using $41(0.284 \mathrm{~g}, 1.0 \mathrm{mmol})$. The product was purified by using petroleum ether-ethyl acetate (10: 1). Yield $0.267 \mathrm{~g}(68 \%)$, yellowish solid, m.p. $72-73^{\circ} \mathrm{C}$. IR (ATR) $v_{\max }=1755,1722,1344$, $1198,1084,505 \mathrm{~cm}^{-1} .{ }^{1} \mathrm{H}$ NMR $\left(400 \mathrm{MHz}, \mathrm{CDCl}_{3}\right) \delta=1.28\left(3 \mathrm{H}, \mathrm{t}, J=7.0 \mathrm{~Hz}, \mathrm{OCH}_{2} \mathrm{C}_{3}\right), 4.25(2 \mathrm{H}, \mathrm{q}, J$ $\left.=7.0 \mathrm{~Hz}, \mathrm{OCH}_{2}\right), 4.87\left(2 \mathrm{H}, \mathrm{s}, \mathrm{OCH}_{2}\right), 7.42(2 \mathrm{H}, \mathrm{d}, J=8.7 \mathrm{~Hz}, \mathrm{H} \mathrm{Ar}), 7.51(2 \mathrm{H}, \mathrm{d}, J=8.6 \mathrm{~Hz}, \mathrm{H} \mathrm{Ar}) \mathrm{ppm}$; ${ }^{13} \mathrm{C} \mathrm{NMR}\left(101 \mathrm{MHz}, \mathrm{CDCl}_{3}\right) \delta=14.1\left(\mathrm{CH}_{3}\right), 61.6\left(\mathrm{OCH}_{2} \mathrm{CH}_{3}\right), 61.7\left(\mathrm{COOCH}_{2}\right), 124.0(\mathrm{CCl}), 128.1(\mathrm{CCl})$, $130.5(2 \times \mathrm{CH}), 134.6(2 \times \mathrm{CH}), 136.7(\mathrm{SC} \mathrm{Ar}), 153.5(\mathrm{NC}), 158.6,160.9(\mathrm{C}=\mathrm{O}), 166.8(\mathrm{COOEt}) ; \mathrm{MS} \mathrm{m} / \mathrm{z}$ $\left(I_{\text {rel }}, \%\right): 391\left[\mathrm{M}^{+}\right]$(48), $288\left[\mathrm{M}-\mathrm{OCH}_{2} \mathrm{CO}_{2} \mathrm{Et}\right]^{+}(50), 225\left[\mathrm{M}-\mathrm{CO}_{2} \mathrm{CH}_{2} \mathrm{CO}_{2} \mathrm{Et}-\mathrm{Cl}\right]^{+}(70), 144[\mathrm{ClPhSH}]^{+}$ (100); HRMS (ESI ${ }^{+}$) $\mathrm{m} / z$ calcd for $\mathrm{C}_{14} \mathrm{H}_{11} \mathrm{NO}_{4} \mathrm{Cl}_{2} \mathrm{~S}_{2} \mathrm{Na}[\mathrm{M}+\mathrm{Na}]^{+}$: 413.9404; found: 413.9405 .

Synthesis of 5-methyl-5-phenyl-3-(trichloroethenyl)-4,5-dihydro-1,2-oxazole (47) (General method). To a solution of nitrodiene $\mathbf{1}(0.271 \mathrm{~g}, 1.0 \mathrm{mmol})$ in dry toluene $(10 \mathrm{~mL})$ prop-1-en-2-ylbenzene $(1.182 \mathrm{~g}, 10.0$ mmol), 18-crown-6 (0.066 g, $0.25 \mathrm{mmol})$, activated molar sieves $4 \AA$ ( $0.250 \mathrm{~g})$, and powdered $\mathrm{NaOH}$ $(0.120 \mathrm{~g}, 3.0 \mathrm{mmol})$ were added under nitrogen atmosphere. The mixture was then stirred at $60^{\circ} \mathrm{C}$ for $16 \mathrm{~h}$, and at $80^{\circ} \mathrm{C}$ for another $16 \mathrm{~h}$. After completion of the reaction, the solvent was evaporated, $10 \%$ aq. $\mathrm{HCl}(10 \mathrm{~mL})$ was added, and the mixture was extracted with DCM $(3 \times 10 \mathrm{~mL})$. The crude product was purified by column chromatography using petroleum ether-ethyl acetate (10: 1). Yield $0.221 \mathrm{~g}(76 \%)$, yellow viscous oil. IR (ATR) $v_{\max }=2977,1684,1446,1265,859,697 \mathrm{~cm}^{-1} .{ }^{1} \mathrm{H} \mathrm{NMR}$ $\left(400 \mathrm{MHz}, \mathrm{CDCl}_{3}\right) \delta=1.77\left(3 \mathrm{H}, \mathrm{s}, \mathrm{CH}_{3}\right), 3.43\left(1 \mathrm{H}, \mathrm{d}, J=16.9 \mathrm{~Hz}, \mathrm{ONCCH}_{2}\right), 3.50(1 \mathrm{H}, \mathrm{d}, J=16.9 \mathrm{~Hz}$, 
$\left.\mathrm{ONCH}_{2}\right), 7.37-7.48(5 \mathrm{H}, \mathrm{m}, \mathrm{H} \mathrm{Ph}) \mathrm{ppm} ;{ }^{13} \mathrm{C} \mathrm{NMR}\left(100 \mathrm{MHz}, \mathrm{CDCl}_{3}\right) \delta=27.8\left(\mathrm{CH}_{3}\right), 50.1\left(\mathrm{CH}_{2}\right), 90.3$ $\left(\mathrm{OCCH}_{3}\right), 121.7,124.5(2 \times \mathrm{CH}), 127.7(\mathrm{CH}), 128.6(2 \times \mathrm{CH}), 137.1\left(\mathrm{CCl}_{2}\right), 144.2,152.5(\mathrm{NCCCl}) \mathrm{ppm}$;

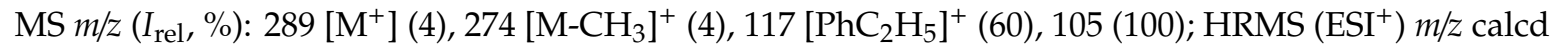
for $\mathrm{C}_{12} \mathrm{H}_{10} \mathrm{NOCl}_{3} \mathrm{Na}[\mathrm{M}+\mathrm{Na}]^{+}$: 311.9726; found: 311.9725 .

tert-Butyl 3-(trichloroethenyl)-4,5-dihydro-1,2-oxazole-5-carboxylate (48). Same procedure as for 47, but using tert-butyl prop-2-enoate $(1.282 \mathrm{~g}, 10.0 \mathrm{mmol})$. Yield $0.195 \mathrm{~g}(65 \%)$, yellowish oil. IR (ATR) $v_{\max }=$ 2981, 1732, 1368, 1232, 1150, $838 \mathrm{~cm}^{-1} .{ }^{1} \mathrm{H}$ NMR $\left(400 \mathrm{MHz} \mathrm{CDCl}_{3}\right) \delta=1.47\left(9 \mathrm{H}, \mathrm{s}, \mathrm{CCH}_{3}\right), 3.53(2 \mathrm{H}, \mathrm{d}$, $\left.J=9.4 \mathrm{~Hz}, \mathrm{ONCCH}_{2}\right), 5.03(1 \mathrm{H}, \mathrm{t}, J=9.4 \mathrm{~Hz}, \mathrm{OCH}) \mathrm{ppm} ;{ }^{13} \mathrm{C} \mathrm{NMR}\left(100 \mathrm{MHz}, \mathrm{CDCl}_{3}\right) \delta=27.8(3 \times$ $\left.\mathrm{CH}_{3}\right), 40.0\left(\mathrm{CH}_{2}\right), 80.2(\mathrm{OCH}), 83.1\left(\mathrm{OCMe}_{3}\right), 120.5(\mathrm{CCl}), 125.2\left(\mathrm{CCl}_{2}\right), 152.3(\mathrm{ONC}), 168.0(\mathrm{C}=\mathrm{O}) \mathrm{ppm}$; ${ }^{15} \mathrm{~N}$ NMR (43.4 MHz, $\mathrm{CDCl}_{3}$, doped with nitromethane (0.0 ppm)) $\delta=7.8 \mathrm{ppm}$; MS $\mathrm{m} / z\left(I_{\text {rel }}, \%\right): 299$ $\left[\mathrm{M}^{+}\right](12), 200\left[\mathrm{M}-\mathrm{CO}_{2} \mathrm{CMe}_{3}\right]^{+}(100), 170\left[\mathrm{M}-\mathrm{C}_{2} \mathrm{Cl}_{3}\right]^{+}(12), 129\left[\mathrm{C}_{2} \mathrm{Cl}_{3}\right]^{+}$(52), $100\left[\mathrm{CO}_{2} \mathrm{CMe}_{3}-\mathrm{H}\right]^{+}$(95); HRMS (ESI ${ }^{+}$) $\mathrm{m} / z$ calcd for $\mathrm{C}_{10} \mathrm{H}_{12} \mathrm{NO}_{3} \mathrm{Cl}_{3} \mathrm{Na}[\mathrm{M}+\mathrm{Na}]^{+}$: 321.9781; found: 321.9781.

2-Ethylhexyl 3-(trichloroethenyl)-4,5-dihydro-1,2-oxazole-5-carboxylate (49). Same procedure as for 47, but using 2-ethylhexyl prop-2-enoate (1.843 g, $10.0 \mathrm{mmol})$. Yield $0.216 \mathrm{~g} \mathrm{(60 \% ),} \mathrm{yellowish} \mathrm{oil.} \mathrm{IR} \mathrm{(ATR)}$ $v_{\max }=2958,2860,1732,1461,1162,851 \mathrm{~cm}^{-1} .{ }^{1} \mathrm{H} \mathrm{NMR}\left(400 \mathrm{MHz}, \mathrm{CDCl}_{3}\right) \delta=0.86-0.88(6 \mathrm{H}, \mathrm{m}, 2 \times$ $\left.\mathrm{CH}_{3}\right), 1.19-1.42\left(8 \mathrm{H}, \mathrm{m}, 4 \times \mathrm{CH}_{2}\right), 1.60-1.64(1 \mathrm{H}, \mathrm{m}, \mathrm{CH}), 3.60\left(1 \mathrm{H}, \mathrm{d}, J=10.7 \mathrm{~Hz}, \mathrm{ONCCH}_{2}\right), 3.61(1 \mathrm{H}$, $\left.\mathrm{d}, J=7.8 \mathrm{~Hz}, \mathrm{ONCCH}_{2}\right), 4.06-4.18\left(2 \mathrm{H}, \mathrm{m}, \mathrm{OCH}_{2}\right), 5.17(1 \mathrm{H}, \mathrm{dd}, J=10.7 \mathrm{~Hz}, J=7.7 \mathrm{~Hz}, \mathrm{OCH}) \mathrm{ppm} ;{ }^{13} \mathrm{C}$ $\operatorname{NMR}\left(100 \mathrm{MHz}, \mathrm{CDCl}_{3}\right) \delta=10.9\left(\mathrm{CH}_{3}\right), 14.0\left(\mathrm{CH}_{3}\right), 22.9\left(\mathrm{CH}_{2}\right), 23.6\left(\mathrm{CH}_{2}\right), 28.8\left(\mathrm{CH}_{2}\right), 30.2\left(\mathrm{CH}_{2}\right), 28.6$ $(\mathrm{CH}), 40.2\left(\mathrm{ONCCH}_{2}\right), 68.5\left(\mathrm{OCH}_{2}\right), 79.6(\mathrm{OCH}), 120.4(\mathrm{CCl}), 125.5\left(\mathrm{CCl}_{2}\right), 152.4(\mathrm{ONC}), 169.2(\mathrm{C}=\mathrm{O})$

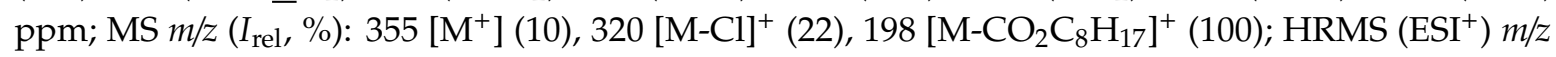
calcd for $\mathrm{C}_{14} \mathrm{H}_{20} \mathrm{NO}_{3} \mathrm{Cl}_{3} \mathrm{Na}[\mathrm{M}+\mathrm{Na}]^{+}$: 378.0407; found: 378.0406 .

3-(Trichloroethenyl)-3a,4,5,6,7,7a-hexahydro-1,2-benzoxazole (50). Same procedure as for 47, but using cyclohexene $(0.821 \mathrm{~g}, 10.0 \mathrm{mmol})$. Yield $0.104 \mathrm{~g}(41 \%)$, yellowish oil. IR (ATR) $v_{\max }=2935,2863,1448$, $912,838,739 \mathrm{~cm}^{-1} .{ }^{1} \mathrm{H}$ NMR $\left(400 \mathrm{MHz}, \mathrm{CDCl}_{3}\right) \delta=1.23-1.43\left(2 \mathrm{H}, \mathrm{m}, \mathrm{CH}_{2}\right), 1.48-1.64\left(3 \mathrm{H}, \mathrm{m}, \mathrm{CH}_{2}\right)$, 1.72-1.82 (1H, m, CH $\left.\mathrm{CH}_{2}\right), 1.83-1.93\left(1 \mathrm{H}, \mathrm{m}, \mathrm{CH}_{2}\right), 2.00-2.09\left(1 \mathrm{H}, \mathrm{m}, \mathrm{CH}_{2}\right), 3.32-3.41(1 \mathrm{H}, \mathrm{m}, \mathrm{CH}), 4.58$ $(1 \mathrm{H}, \mathrm{ddd}, J=8.2 \mathrm{~Hz}, J=4.2 \mathrm{~Hz}, J=4.2 \mathrm{~Hz}, \mathrm{CH}) \mathrm{ppm} ;{ }^{13} \mathrm{C}\left(100 \mathrm{MHz}, \mathrm{CDCl}_{3}\right) \delta=19.9\left(\mathrm{CH}_{2}\right), 21.7\left(\mathrm{CH}_{2}\right)$, $24.8\left(\mathrm{CH}_{2}\right), 25.3\left(\mathrm{CH}_{2}\right), 46.1(\mathrm{CH}), 81.4(\mathrm{CH}), 121.0(\mathrm{CCl}), 124.4\left(\mathrm{CCl}_{2}\right), 159.1(\mathrm{ONC}) \mathrm{ppm} ; \mathrm{MS} \mathrm{m} / \mathrm{z}\left(\mathrm{I}_{\mathrm{rel}}\right.$,

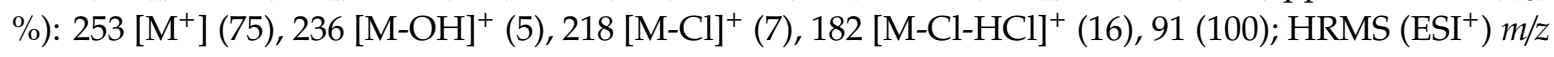
calcd for $\mathrm{C}_{9} \mathrm{H}_{10} \mathrm{NOCl}_{3} \mathrm{Na}[\mathrm{M}+\mathrm{Na}]^{+}:$275.9726; found: 275.9725 .

Synthesis of 1-[1,1-dichloro-3-(1,3-dithiolan-2-ylidene)-3-nitroprop-1-en-2-yl]-4-(4-fluorophenyl)pipera- zine (52). A solution of dithiolane $51(0.292 \mathrm{~g}, 1.0 \mathrm{mmol})$ in $\mathrm{MeOH}(10 \mathrm{~mL})$ and 1-(4-fluorophenyl)piperazine $(0.433 \mathrm{~g}, 2.4 \mathrm{mmol})$ was stirred at reflux for $7 \mathrm{~d}$. After cooling, the solution was concentrated and treated with water for $30 \mathrm{~min}$. The resulting precipitate was filtered off and washed with water $(2 \times 5$ $\mathrm{mL})$. Yield $0.393 \mathrm{~g}(90 \%)$, orange solid, m.p. $47-50{ }^{\circ} \mathrm{C}$. IR $(\mathrm{KBr}) v_{\max }=2825,1509,1272,1226,954,816$ $\mathrm{cm}^{-1} .{ }^{1} \mathrm{H}$ NMR $\left(200 \mathrm{MHz}, \mathrm{CDCl}_{3}\right) \delta=3.08-3.39\left(8 \mathrm{H}, \mathrm{m}, \mathrm{NCH}_{2}\right), 3.48-3.61\left(4 \mathrm{H}, \mathrm{m}, \mathrm{SCH}_{2}\right), 6.80-7.11$ $(4 \mathrm{H}, \mathrm{m}, \mathrm{H} \mathrm{Ar}) \mathrm{ppm} ;{ }^{13} \mathrm{C} \mathrm{NMR}\left(50 \mathrm{MHz}, \mathrm{CDCl}_{3}\right) \delta=37.6\left(\mathrm{SCH}_{2}\right), 40.1\left(\mathrm{SCH}_{2}\right), 49.2\left(2 \times \mathrm{NCH}_{2}\right), 50.6(2$ $\left.\times \mathrm{NCH}_{2}\right), 113.3\left(\mathrm{CCl}_{2}\right), 115.5\left(\mathrm{~J}_{\mathrm{C}, \mathrm{F}}=21.9 \mathrm{~Hz}, 2 \times \mathrm{CH}\right), 118.2\left(\mathrm{~J}_{\mathrm{C}, \mathrm{F}}=7.7 \mathrm{~Hz}, 2 \times \mathrm{CH}\right), 130.9\left(\mathrm{CNO}_{2}\right), 139.0$ $(\mathrm{CN}), 147.9\left(J_{\mathrm{C}, \mathrm{F}}=2.2 \mathrm{~Hz}, \mathrm{NC}\right.$ Ar), $157.3\left(J_{\mathrm{C}, \mathrm{F}}=238.9 \mathrm{~Hz}, \mathrm{CF}\right), 170.4(\mathrm{SCS}) \mathrm{ppm}$; MS m/z (I $\left[\mathrm{M}^{+}\right]$(25), $418[\mathrm{M}-\mathrm{OH}]^{+}(4), 389\left[\mathrm{M}-\mathrm{NO}_{2}\right]^{+}$(7), 179 [F-Ph-piperazine] $^{+}$(15), 122 (100); HRMS m/z calcd for $\mathrm{C}_{16} \mathrm{H}_{16} \mathrm{~N}_{3} \mathrm{O}_{2} \mathrm{FCl}_{2} \mathrm{~S}_{2}[\mathrm{M}]^{+}$: 435.0045; found: 435.0047 .

Synthesis of 1-[2-Chloro-5-(ethenylsulfanyl)-4-nitrothiophen-3-yl]-4-(4-fluorophenyl)piperazine (53). An aqueous solution of $\mathrm{NaOH}(3.0 \mathrm{mmol}, 40 \%)$ was added dropwise to a solution of dithiolane $52(0.436 \mathrm{~g}$, $1.0 \mathrm{mmol})$ in DMSO $(10 \mathrm{~mL})$ at $0{ }^{\circ} \mathrm{C}$. After $1 \mathrm{~h}$ at $0{ }^{\circ} \mathrm{C}$, the mixture was stirred at r.t. for an additional 3 h. Subsequently, cold water was added and the mixture was treated dropwise with diluted $\mathrm{HCl}$ until a precipitate formed. The precipitate was filtered off and washed with water $(2 \times 5 \mathrm{~mL})$ and cold $\mathrm{MeOH}$ (3 mL). Yield $0.340 \mathrm{~g}(85 \%)$, yellowish solid, m.p. 83-86 ${ }^{\circ} \mathrm{C}$. IR $(\mathrm{KBr}) v_{\max }=2829,1543,1511,1322$, $1233,831 \mathrm{~cm}^{-1} .{ }^{1} \mathrm{H}$ NMR $\left(200 \mathrm{MHz}, \mathrm{CDCl}_{3}\right) \delta=3.16-3.26\left(4 \mathrm{H}, \mathrm{m}, \mathrm{NCH}_{2}\right), 3.31-3.47\left(4 \mathrm{H}, \mathrm{m}, \mathrm{NCH}_{2}\right)$, $5.83\left(1 \mathrm{H}, \mathrm{d}, J=9.1 \mathrm{~Hz}, \mathrm{SCHC} \underline{\mathrm{H}}_{2}\right), 8.55\left(1 \mathrm{H}, \mathrm{d}, J=5.9 \mathrm{~Hz}, \mathrm{SCHC}_{2}\right), 6.55(1 \mathrm{H}, \mathrm{dd}, J=16.4 \mathrm{~Hz}, J=9.2$ 
$\mathrm{Hz}, \mathrm{SCH}), 6.86-7.09\left(4 \mathrm{H}, \mathrm{m}, \mathrm{H}\right.$ Ar) ppm; ${ }^{13} \mathrm{C} \mathrm{NMR}\left(50 \mathrm{MHz}, \mathrm{CDCl}_{3}\right) \delta=50.0\left(2 \times \mathrm{NCH}_{2}\right), 51.5(2 \times$ $\left.\mathrm{NCH}_{2}\right), 115.6\left(J_{\mathrm{C}, \mathrm{F}}=21,9 \mathrm{~Hz}, 2 \times \mathrm{CH}\right), 118.6\left(J_{\mathrm{C}, \mathrm{F}}=8.1 \mathrm{~Hz}, 2 \times \mathrm{CH}\right), 119.1(\mathrm{CCl}), 126.2\left(\mathrm{CH}_{2}\right.$ vin $), 126.8$ (CH vin), $139.4\left(\mathrm{CNO}_{2}\right), 141.0(\mathrm{CN}), 143.3(\mathrm{SCS}), 148.5\left(J_{\mathrm{C}, \mathrm{F}}=3.7 \mathrm{~Hz}, \mathrm{NC} \mathrm{Ar}\right), 157.7\left(J_{\mathrm{C}, \mathrm{F}}=234.5 \mathrm{~Hz}\right.$, CF) ppm; MS m/z (I $I_{\text {rel }} \%$ ): $399\left[\mathrm{M}^{+}\right]$(85), $352\left[\mathrm{M}-\mathrm{HNO}_{2}\right]^{+}$(17), 317 [M-HNO $\left._{2}-\mathrm{Cl}\right]^{+}$(4), 122 (100); HRMS $\left(\mathrm{ESI}^{+}\right) \mathrm{m} / \mathrm{z}$ calcd for $\mathrm{C}_{16} \mathrm{H}_{15} \mathrm{~N}_{3} \mathrm{O}_{2} \mathrm{ClFS}_{2}[\mathrm{M}+\mathrm{H}]^{+}$: 399.0278; found: 399.0278 .

Synthesis of 5-(Ethenylsulfanyl)-3-[4-(4-fluorophenyl)piperazin-1-yl]-4-nitrothiophene-2-carbaldehyde (54). A solution of thiophene $53(0.400 \mathrm{~g}, 1.0 \mathrm{mmol})$ and $\mathrm{POCl}_{3}(0.307 \mathrm{~g}, 2.0 \mathrm{mmol})$ in dry DMF was stirred at r.t. for $1 \mathrm{~d}$ and then at $55^{\circ} \mathrm{C}$ for $2 \mathrm{~d}$. After cooling down to $0{ }^{\circ} \mathrm{C}$, cold water $(15 \mathrm{~mL})$ was added and the mixture extracted with chloroform $(3 \times 10 \mathrm{~mL})$. After drying over $\mathrm{CaCl}_{2}$, the crude product was purified by column chromatography using petroleum ether-ethyl acetate (2: 1). Yield $0.252 \mathrm{~g}(64 \%)$, orange solid, m.p. $104-105^{\circ} \mathrm{C}$. IR (ATR) $v_{\max }=2833,1621,1531,1505,1223,997 \mathrm{~cm}^{-1} .{ }^{1} \mathrm{H}$ NMR $(400$ $\left.\mathrm{MHz}, \mathrm{CDCl}_{3}\right) \delta=3.25-3.33\left(4 \mathrm{H}, \mathrm{m}, \mathrm{NCH}_{2}\right), 3.50-3.59\left(4 \mathrm{H}, \mathrm{m}, \mathrm{OCH}_{2}\right), 5.99(2 \mathrm{H}, \mathrm{dd}, J=16.7 \mathrm{~Hz}, J=9.4$ $\mathrm{Hz}, \mathrm{CH}_{2}$ vin $), 6.65(1 \mathrm{H}, \mathrm{dd}, J=16.6 \mathrm{~Hz}, J=9.3 \mathrm{~Hz}, \mathrm{SCH}$ vin $), 6.88-7.03(4 \mathrm{H}, \mathrm{m}, \mathrm{H} \mathrm{Ar}), 10.03(1 \mathrm{H}, \mathrm{s}$, $\mathrm{CHO}) \mathrm{ppm} ;{ }^{13} \mathrm{C} \mathrm{NMR}\left(100 \mathrm{MHz}, \mathrm{CDCl}_{3}\right) \delta=50.7\left(2 \times \mathrm{NCH}_{2}\right), 53.5\left(2 \times \mathrm{OCH}_{2}\right), 115.6,115.7\left(\mathrm{~J}_{\mathrm{C}, \mathrm{F}}=\right.$ $22.2 \mathrm{~Hz}, 2 \times \mathrm{CH}$ Ar), $118.6\left(J_{\mathrm{C}, \mathrm{F}}=7.7 \mathrm{~Hz}, 2 \times \mathrm{CH} \mathrm{Ar}\right), 124.7(\mathrm{CN}), 125.6(\mathrm{SCH}), 128.7\left(\mathrm{CH}_{2} \mathrm{vin}\right), 136.2$ $\left(\mathrm{CNO}_{2}\right), 147.6\left(J_{\mathrm{C}, \mathrm{F}}=2.2 \mathrm{~Hz}, \mathrm{NC} \mathrm{Ar}\right), 150.5(\mathrm{SCS}), 157.6\left(J_{\mathrm{C}, \mathrm{F}}=239.9 \mathrm{~Hz}, \mathrm{CF}\right), 159.8(\mathrm{SC}), 180.2(\mathrm{CHO})$ ppm; MS $m / z\left(I_{\text {rel }} \% \text { \%): } 393\left[\mathrm{M}^{+}\right](15), 376[\mathrm{M}-\mathrm{OH}]^{+}(5), 346\left[\mathrm{M}-\mathrm{HNO}_{2}\right]^{+}(3), 179 \text { [piperazine-Ph-F }\right]^{+}$ (7), 122 (100); $\mathrm{HRMS}\left(\mathrm{ESI}^{+}\right) \mathrm{m} / \mathrm{z}$ calcd for $\mathrm{C}_{17} \mathrm{H}_{16} \mathrm{~N}_{3} \mathrm{FO}_{3} \mathrm{~S}_{2} \mathrm{Na}[\mathrm{M}+\mathrm{Na}]^{+}: 416.0515$; found: 416.0514.

Synthesis of \{[5-(Ethenylsulfonyl)-3-(morpholin-4-yl)-4-nitrothiophen-2-yl]methylidene $\}$ propanedinitrile (57). A solution of thiophene $56(0.348 \mathrm{~g}, 1.0 \mathrm{mmol})$ and hydrogen peroxide $(3.0 \mathrm{mmol}, 35 \%)$ in acetic acid was stirred at $50-55{ }^{\circ} \mathrm{C}$ for $3 \mathrm{~h}$. After cooling, ice was poured into the mixture and the resulting precipitate was filtered off and washed with water $(3 \times 5 \mathrm{~mL})$. Yield $0.312 \mathrm{~g}(82 \%)$, red solid, m.p. 121-123 ${ }^{\circ} \mathrm{C}$. IR (KBr) $v_{\max }=2857,2223(\mathrm{CN}), 1541,1340,1111,857 \mathrm{~cm}^{-1} .{ }^{1} \mathrm{H}$ NMR $\left(200 \mathrm{MHz}, \mathrm{CDCl}_{3}\right) \delta$ $=3.23-3.30\left(4 \mathrm{H}, \mathrm{m}, \mathrm{NCH}_{2}\right), 3.84-3.90\left(4 \mathrm{H}, \mathrm{m}, \mathrm{OCH}_{2}\right), 6.15\left(1 \mathrm{H}, \mathrm{dd}, J=9.3, J=1.4, \mathrm{CH}_{2, \text { cis }}\right.$ vin $), 6.45(1 \mathrm{H}$, $\mathrm{dd}, J=16.2, J=1.3, \mathrm{CH}_{2}$, trans vin), $7.09(1 \mathrm{H}, \mathrm{dd}, J=16.3, J=9.3, \mathrm{CH}$ vin $), 8.02(1 \mathrm{H}, \mathrm{s}, \mathrm{SCCH}) .{ }^{13} \mathrm{C} \mathrm{NMR}$ $\left(50 \mathrm{MHz}, \mathrm{CDCl}_{3}\right) \delta=52.0\left(2 \times \mathrm{NCH}_{2}\right), 67.0\left(2 \times \mathrm{OCH}_{2}\right), 80.3\left(\underline{\mathrm{C}}(\mathrm{CN})_{2}\right), 112.2(2 \times \mathrm{CN}), 113.3,124.1$ ( $\mathrm{CH}_{2}$ vin), 127.4 (SC), 138.5 ( $\mathrm{CH}$ vin), $146.8(\mathrm{SCCH}), 151.4$ (C-morph), $166.1\left(\mathrm{SO}_{2} \mathrm{C}\right), \mathrm{CNO}_{2}$ could not be detected. Mass spectrum, $m / z\left(I_{\text {rel }}, \%\right): 380\left[\overline{\mathrm{M}}^{+}\right](3), 363[\mathrm{M}-\mathrm{OH}]^{+}(20), 346[\mathrm{M}-2(\mathrm{OH})]^{+}(100), 289$

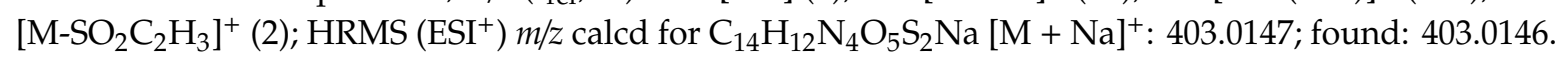

Synthesis of 2,2'-\{[5-(Ethenylsulfanyl)-3-(morpholin-4-yl)-4-nitrothiophen-2-yl]methanediyl\}bis(5,5di-methylcyclohexane-1,3-dione) (58). A solution of thiophene $55(0.300 \mathrm{~g}, 1.0 \mathrm{mmol})$, dimedone (0.308 g, $2.2 \mathrm{mmol})$ and pyridine $(8.0 \mathrm{mg}, 0.1 \mathrm{mmol})$ in $\mathrm{MeOH}(15 \mathrm{~mL})$ was stirred at r.t. for $1 \mathrm{~d}$ and then at $35-40{ }^{\circ} \mathrm{C}$ for $2 \mathrm{~d}$. After cooling to r.t., the mixture was treated with diluted $\mathrm{HCl}$ and the resulting precipitate was filtered off and washed with water $(2 \times 5 \mathrm{~mL})$ and cold $\mathrm{Et}_{2} \mathrm{O}(3 \mathrm{~mL})$. Yield $0.411 \mathrm{~g}$ (73\%), yellow solid, m.p. $159-160{ }^{\circ} \mathrm{C}$. IR $(\mathrm{KBr}) v_{\max }=3243,2961,1717,1626,1542,1068 \mathrm{~cm}^{-1} .{ }^{1} \mathrm{H}$ NMR $\left(200 \mathrm{MHz}, \mathrm{CDCl}_{3}\right) \delta=1.03\left(12 \mathrm{H}, \mathrm{s}, 4 \mathrm{CH}_{3}\right), 2.34\left(8 \mathrm{H}, \mathrm{s}, 4 \mathrm{COCH}_{2}\right), 2.60-3.80\left(10 \mathrm{H}, \mathrm{m}, 2 \mathrm{NCH}_{2}, 2 \mathrm{OCH}_{2}\right.$, $2 \mathrm{COCH}), 5.77\left(2 \mathrm{H}, \mathrm{dd}, J=16.4 \mathrm{~Hz}, J=9.3 \mathrm{~Hz}, \mathrm{CH}_{2}\right.$ vin $), 6.41-6.51(1 \mathrm{H}, \mathrm{m}, \mathrm{SCH}), 6.67(1 \mathrm{H}, \mathrm{dd}, J=$ $16.4 \mathrm{~Hz}, J=9.3 \mathrm{~Hz}, \mathrm{CH}$ vin) ppm; ${ }^{13} \mathrm{C} \mathrm{NMR}\left(50 \mathrm{MHz}, \mathrm{CDCl}_{3}\right) \delta=26.7(\mathrm{SCC} H), 28.1\left(2 \times \mathrm{CH}_{3} \underline{\mathrm{C}}\right), 31.6$ $\left(4 \times \mathrm{CH}_{3}\right), 46.7\left(2 \times \mathrm{NCH}_{2}\right), 49.6\left(4 \times \mathrm{COCH}_{2}\right), 67.4\left(2 \times \mathrm{OCH}_{2}\right), 77.2(2 \times \underline{\mathrm{CC}}=\mathrm{O}), 116.1(\mathrm{SC}), 124.4$ $\left(\mathrm{CH}_{2}\right.$ vin), $127.6(\mathrm{SCH}), 137.2\left(\mathrm{CNO}_{2}\right), 141.1$ (C-morph), $143.2(\mathrm{SCS}), 189.9(4 \times \mathrm{C}=\mathrm{O}) \mathrm{ppm} ; \mathrm{MS} \mathrm{m} / \mathrm{z}\left(I_{\mathrm{rel}}\right.$, \%): $562\left[\mathrm{M}^{+}\right](12), 543\left[\mathrm{M}-\mathrm{H}_{2} \mathrm{O}-\mathrm{H}\right]^{+}(12), 526\left[\mathrm{M}-2\left(\mathrm{H}_{2} \mathrm{O}\right)\right]^{+}(42), 458\left[\mathrm{M}-\mathrm{SC}_{2} \mathrm{H}_{3}-\mathrm{NO}_{2}+\mathrm{H}\right]^{+}$(10); $\mathrm{HRMS}$ $\left(\mathrm{ESI}^{+}\right) \mathrm{m} / z$ calcd for $\mathrm{C}_{27} \mathrm{H}_{34} \mathrm{~N}_{2} \mathrm{O}_{7} \mathrm{~S}_{2} \mathrm{Na}[\mathrm{M}+\mathrm{Na}]^{+}$: 585.1705; found: 585.1705.

Synthesis of 4-[5-(Ethenylsulfanyl)-4-nitro-2-\{[2-(2,4,6-trichlorophenyl)hydrazinylidene]methyl\} thiophen-3-yl]morpholine (59). A solution of thiophene $55(0.300 \mathrm{~g}, 1.0 \mathrm{mmol})$ and (2,4,6-trichlorophenyl)-hydrazine $(0.634 \mathrm{~g}, 3.0 \mathrm{mmol})$ in $\mathrm{MeOH}(15 \mathrm{~mL})$ was refluxed for $3 \mathrm{~h}$. After cooling to r.t., the mixture was treated with diluted $\mathrm{HCl}$ and the resulting precipitate was filtered off and washed with water $(2 \times 5 \mathrm{~mL})$ and cold $\mathrm{MeOH}(3 \mathrm{~mL})$. Yield $0.425 \mathrm{~g}(86 \%)$, orange solid, m.p. $192-194{ }^{\circ} \mathrm{C}$. IR $(\mathrm{KBr}) v_{\max }=3228,1520,1445,1332,1107,851 \mathrm{~cm}^{-1} .{ }^{1} \mathrm{H} \mathrm{NMR}\left(200 \mathrm{MHz}, \mathrm{CDCl}_{3}\right) \delta=$ 
3.05-3.19 (4H, m, NCH $), 3.75-3.82\left(4 \mathrm{H}, \mathrm{m}, \mathrm{OCH}_{2}\right), 5.87\left(2 \mathrm{H}, \mathrm{dd}, J=16.7 \mathrm{~Hz}, J=9.4 \mathrm{~Hz}, \mathrm{CH}_{2}\right.$ vin), 6.65 $(1 \mathrm{H}, \mathrm{dd}, J=16.7 \mathrm{~Hz}, J=9.4 \mathrm{~Hz}, \mathrm{SCH}$ vin $), 7.36(2 \mathrm{H}, \mathrm{s}, \mathrm{H}$ Ar $), 7.64(1 \mathrm{H}, \mathrm{br} \mathrm{s}, \mathrm{NH}), 8.01(1 \mathrm{H}, \mathrm{s}, \mathrm{NCH})$ ppm; ${ }^{13} \mathrm{C}\left(50 \mathrm{MHz}, \mathrm{CDCl}_{3}\right) \delta=51.0\left(2 \times \mathrm{NCH}_{2}\right), 67.5\left(2 \times \mathrm{OCH}_{2}\right), 126.4\left(\mathrm{CH}_{2}\right.$ vin $), 126.8(\mathrm{SCH}$ vin $)$, $127.6(2 \times \mathrm{CCl}), 129.0(2 \times \mathrm{CH}), 129.1(\mathrm{CCl}), 130.1(\mathrm{SC}), 133.3(\mathrm{NCH}), 135.8(\mathrm{NHC}), 139.5\left(\mathrm{CNO}_{2}\right), 142.3$

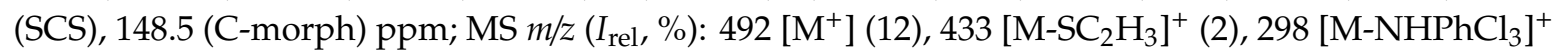
(55), $195\left[\mathrm{H}_{2} \mathrm{NPhCl}_{3}\right]^{+}$(100); HRMS $\left(\mathrm{ESI}^{+}\right) \mathrm{m} / z$ calcd for $\mathrm{C}_{17} \mathrm{H}_{15} \mathrm{~N}_{4} \mathrm{O}_{3} \mathrm{Cl}_{3} \mathrm{~S}_{2} \mathrm{Na}[\mathrm{M}+\mathrm{Na}]^{+}: 514.9549$; found: 514.9547.

Synthesis of 5-\{[5-(Ethenylsulfanyl)-3-(morpholin-4-yl)-4-nitrothiophen-2-yl]methylidene\}-2,2-dimethyl1,3-dioxane-4,6-dione (60). A solution of thiophene $55(0.300 \mathrm{~g}, 1.0 \mathrm{mmol})$, 2,2-dimethyl-1,3-dioxane-4,6-dione $(0.288 \mathrm{~g}, 2.0 \mathrm{mmol})$ and piperidine $(8.5 \mathrm{mg}, 0.1 \mathrm{mmol})$ in $\mathrm{MeOH}(15 \mathrm{~mL})$ was stirred at r.t. for $1 \mathrm{~d}$. Then another $0.288 \mathrm{~g}$ of 2,2-dimethyl-1,3-dioxane-4,6-dione were added and the mixture was stirred for an additional $2 \mathrm{~d}$. Subsequently, the mixture was treated with diluted $\mathrm{HCl}$ and the resulting precipitate was filtered off and washed with water $(2 \times 5 \mathrm{~mL})$ and cold $\mathrm{MeOH}(3 \mathrm{~mL})$. Yield $0.333 \mathrm{~g}(73 \%)$, red solid, m.p. $173-174{ }^{\circ} \mathrm{C}$. IR (ATR) $v_{\max }=1683,1516,1332$, 1197, 1113, $787 \mathrm{~cm}^{-1} .{ }^{1} \mathrm{H}$ NMR $\left(200 \mathrm{MHz}, \mathrm{CDCl}_{3}\right) \delta=1.76\left(6 \mathrm{H}, \mathrm{s}, \mathrm{CH}_{3}\right), 3.33-3.39\left(4 \mathrm{H}, \mathrm{m}, \mathrm{NCH}_{2}\right)$, 3.93-3.96 $\left(4 \mathrm{H}, \mathrm{m}, \mathrm{OCH}_{2}\right), 6.00\left(2 \mathrm{H}, \mathrm{dd}, J=16.8 \mathrm{~Hz}, J=9.5 \mathrm{~Hz}, \mathrm{CH}_{2}\right.$ vin $), 6.75(1 \mathrm{H}, \mathrm{dd}, J=16.8 \mathrm{~Hz}$, $J=9.5 \mathrm{~Hz}, \mathrm{SCH}$ vin $), 8.68(1 \mathrm{H}, \mathrm{s}, \mathrm{SCCH}) \mathrm{ppm} ;{ }^{13} \mathrm{C}\left(200 \mathrm{MHz}, \mathrm{CDCl}_{3}\right) \delta=27.5\left(2 \times \mathrm{CH}_{3}\right), 53.2(2 \times$ $\left.\mathrm{NCH}_{2}\right), 67.1\left(2 \times \mathrm{OCH}_{2}\right), 103.6\left(\mathrm{CMe}_{2}\right), 104.7(\underline{\mathrm{CC}}=\mathrm{O}), 119.5(\mathrm{SC}), 124.9(\mathrm{SCH}$ vin $), 128.7\left(\mathrm{CH}_{2}\right.$ vin $)$, 137.2 $\left(\mathrm{CNO}_{2}\right), 144.1(\mathrm{SCC} H), 156.9$ (SCS), $161.4(\mathrm{C}-\mathrm{morph}), 162.3(\mathrm{C}=\mathrm{O}), 163.4(\mathrm{C}=\mathrm{O}) \mathrm{ppm} ; \mathrm{MS} \mathrm{m} / \mathrm{z}\left(\mathrm{I}_{\text {rel }}\right.$, $\%): 426\left[\mathrm{M}^{+}\right]$(100), $409[\mathrm{M}-\mathrm{OH}]^{+}$(10), $351\left[\mathrm{M}-\mathrm{Me}_{2} \mathrm{CO}_{2} \mathrm{H}\right]^{+}$(24), $295\left[\mathrm{M}-\mathrm{NO}_{2}-\mathrm{morph}+\mathrm{H}\right]^{+}$(27); HRMS $\left(\mathrm{ESI}^{+}\right) \mathrm{m} / \mathrm{z}$ calcd for $\mathrm{C}_{17} \mathrm{H}_{18} \mathrm{~N}_{2} \mathrm{O}_{7} \mathrm{~S}_{2} \mathrm{Na}[\mathrm{M}+\mathrm{Na}]^{+}$: 449.0453; found: 449.0453 .

\subsection{Evaluation of Biological Activity}

For a first evaluation of the biological activity of heterocycles, the influence of the compounds on the growth of bacteria, namely of the Gram-positive strain Staphylococcus aureus SH-1000 and of the Gram-negative uropathogenic strain Escherichia coli UPEC796, and on the viability of mammalian cells, namely the murine fibroblast cell line L929, was evaluated.

Bacteria were cultivated in the complex Lysogeny broth. An aliquot of an overnight culture was diluted with fresh medium to an $\mathrm{OD}_{600}$ (optical density at $600 \mathrm{~nm}$ ) of $0.1-0.2$. This intermediate culture was incubated at $37^{\circ} \mathrm{C}$ with shaking until an $\mathrm{OD}_{600}=0.5$ was reached to generate a culture of exponentially growing cells. The cells were again diluted with fresh medium to an $\mathrm{OD}_{600}=0.2$ to generate the working culture.

In each well of a 96-well plate, $90 \mu 1$ Lysogeny broth were placed, to which $1.8 \mu 1$ of compound solutions were added. The incubation was started after the addition of $90 \mu \mathrm{l}$ of the working culture, resulting in a total volume of $180 \mu \mathrm{l}$. The microtiter plates were incubated at $37^{\circ} \mathrm{C}$. Bacterial growth was followed by determination of the $\mathrm{OD}_{600}$ with the microplate spectrophotometer PowerWave $\mathrm{e}^{\mathrm{TM}}$ (BioTek; Bad Friedrichshall, Germany) in regular time intervals, starting $2 \mathrm{~h}$ after the inoculation. The final value was obtained after a growth period of $24 \mathrm{~h}$.

L929 cells were cultivated in RPMI cell culture medium, supplemented with 10\% FBS (serum) in a cell culture incubator at $37^{\circ} \mathrm{C}$ and $10 \% \mathrm{CO}_{2} .60 \mu \mathrm{l}$ of a cell suspension with $3 * 10^{4}$ cells $/ \mathrm{mL}$ were seeded in each well of an assay-ready 96 well microtiter plate. The assay-ready microtiter plates were prepared from the $10 \mathrm{mM}$ DMSO stock solutions of the compounds with the Echo ${ }^{\circledR} 525$ acoustic liquid handler (Labcyte Inc., USA). The microtiter plates with compounds and cells were incubated at $37{ }^{\circ} \mathrm{C}, 10 \%$ $\mathrm{CO}_{2}$ in a cell culture incubator for three days. The remaining viability of cells was assessed with the alamarBlue ${ }^{\circledR}$ assay [88] (Thermo Fisher Scientific (Waltham, MA, USA) according to the instructions given by the manufacturer, i.e., $5 \mu \mathrm{l}$ of the resazurin-solution were given in each well of the 96 -well plate and incubated for up to $4 \mathrm{~h}$. The turnover was determined via the fluorescence of resorufin $\left(\lambda_{\mathrm{ex}}=\right.$ $540 \mathrm{~nm} ; \lambda_{\mathrm{em}}=600 \mathrm{~nm}$ ) with the multi-mode microplate reader Synergy ${ }^{\mathrm{TM}} 4$ (BioTek).

The primary evaluation of the biological activity was done with a single concentration of the compounds, which was $100 \mu \mathrm{M}$ in the bacterial assays and $10 \mu \mathrm{M}$ in the cell culture assay. Compounds 
were considered to show activity in these assays, when the residual growth or viability, respectively, was reduced to $50 \%$ or less of the growth or viability of an untreated bacterial or cell culture. The activities of these compounds were validated by investigating the influences of compound concentrations and determinations of the $\mathrm{EC}_{50}$ values (concentration resulting in $50 \%$ of the observed effect). Diluted compound solutions were prepared either by manual serial dilutions using eight channel pipettes or again with the Echo ${ }^{\circledR} 525$ transfering varying volumes from the compound plate to the assay plate.

The $\mathrm{EC}_{50}$ values were determined by nonlinear regression with a 4-parameter equation using the respective module from GraphPadPrism.

\section{Conclusions}

Starting from three polyhalogenated nitro-1,3-butadienes, we developed an efficient and practical strategy for the multigram synthesis of the following heterocycles with unique substitution patterns: benzoxazolines, benzimidazolines, imidazolidines, Imidacloprid analogues, thia-zolidinones, pyrimidines, pyrazoles, $4 H$-pyrido[1,2- $a]$ pyrimidines, benzo[ $h]$ quinolines, isothiazoles, dihydroisoxazoles, and thiophenes. Quite some of these heterocycles deserve interest as key units in synthesis, chemical biology, and medicinal chemistry. Successive synthetic modifications of the heterocycles are predictable and feasible.

Supplementary Materials: The following are available online, Figures S1-S203: ${ }^{1} \mathrm{H}-\mathrm{NMR},{ }^{13} \mathrm{C}-\mathrm{NMR},{ }^{15} \mathrm{~N}$, ${ }^{1} \mathrm{H}-\mathrm{HMBC}-\mathrm{NMR}$, and mass spectra. Figures S204 and S205: Biological profiling of compounds 4a-58.

Author Contributions: Conceptualization, D.E.K. and V.A.Z.; Synthesis and spectroscopic identification of the synthesized compounds, V.A.Z. and S.R.K.; Evaluation of the biological activity of the synthesized compounds, U.B. and I.R.; Writing - original draft, V.A.Z. and U.B.; Writing-review \& editing, D.E.K. and U.B. All authors have read and agreed to the published version of the manuscript.

Funding: This research received no external funding.

Acknowledgments: We thank G. Dräger (Leibniz University Hannover, Germany) and H. Frauendorf (Georg-August University Göttingen, Germany) for extensive HRMS measurements, B. Pawletta (Helmholtz Centre for Infection Research, Germany), S. Daenicke (Helmholtz Centre for Infection Research, Germany), and M. Weigert (Clausthal University of Technology) for technical assistance.

Conflicts of Interest: The authors declare no conflict of interest.

\section{References}

1. Kaberdin, R.V.; Potkin, V.I.; Zapol'skii, V.A. Nitrobutadienes and their halogen derivatives: Synthesis and reactions. Russ. Chem. Rev. 1997, 66, 827-842. [CrossRef]

2. Kaberdin, R.V.; Potkin, V.I.; Zapol'skii, V.A. Synthesis and reactions of mixed halogenbuta-1,3-dienes. Russ. Chem. Rev. 1999, 68, 765-779. [CrossRef]

3. Ol'dekop, Y.A.; Kaberdin, R.V. 2-Nitropentachloro-1,3-butadiene. J. Org. Chem. USSR (Engl. Transl.) 1976, 12, 1986.

4. Potkin, V.I.; Zapol'skii, V.A.; Kaberdin, R.V. Nitration of 2-H-pentachloro-1,3-butadiene. Dokl. Natl. Acad. Sci. Belarus 1996, 40, 68-71.

5. Potkin, V.I.; Gogolinskii, V.I.; Nechai, N.I.; Zapol'skii, V.A.; Kaberdin, R.V. Synthesis and some reactions of 4-bromo-2-nitro-1,1,3,4-tetrachloro-1,3-butadiene. Russ. J. Org. Chem. 1995, 31, 1610-1616.

6. Zapol'skii, V.A.; Potkin, V.I.; Kaberdin, R.V. Synthesis of polyhalobutadienes and their functional derivatives from 1,2-dichloroethylene dimer. Russ. J. Org. Chem. 1994, 30, 1435-1445.

7. Pearson, R.G. Hard and Soft Acids and Bases; Dowden, Hutchinson, and Ross: Stroudsburg, PA, USA, 1973.

8. Ol'dekop, Y.A.; Kaberdin, R.V.; Potkin, V.I.; Shingel, I.A. Synthesis of some heterocyclic compounds based on 2-nitropentachloro-1, 3-butadiene. Chem. Inf. 1979, 15, 276-278.

9. Zapol'skii, V.A.; Namyslo, J.C.; Gjikaj, M.; Kaufmann, D.E. Chemistry of polyhalogenated nitrobutadienes, Part 9: Acyclic and heterocyclic nitroenamines and nitroimines from 2-nitroperchlorobuta-1,3-diene. Z. Naturforsch. 2010, 65b, 843-860. [CrossRef]

10. Ehrhardt, T.; Reindl, A.; Freund, A.; Schmidt, R.M.; Sonnewald, U.; Stitt, N.M.; Lein, W.; Boernke, F.; Deist, K. Clp-Protease as Target for Herbicides. WO 2005054283 A3, 1 September 2005. 
11. Zapol'skii, V.A.; Namyslo, J.C.; Gjikaj, M.; Kaufmann, D.E. Chemistry of polyhalogenated nitrobutadienes, 4: Reactions of mono-, bis-, and tris(4-tolylthio) derivatives of 2-nitroperchloro-1,3-butadiene with $\alpha, \beta$-bifunctional nucleophiles. ARKIVOC 2007. [CrossRef]

12. Fischer, R.; Jeschke, P.; Erdelen-Lubos, A.; Loesel, P.; Reckmann, U.; Kaufmann, D.E.; Zapol'skii, V.A. Halogenated Nitrobutadienes for Controlling Animal Pests. U.S. Patent 7332512 B2, 19 February 2008.

13. Zapol'skii, V.A.; Fischer, R.; Namyslo, J.C.; Kaufmann, D.E. Chemistry of polyhalogenated nitrobutadienes, 8: Nitropolychlorobutadienes-Precursors for insecticidal neonicotinoids. Bioorganic Med. Chem. 2009, 17, 4206-4215. [CrossRef]

14. Gazieva, G.A.; Izmest'Ev, A.N. Oxoindolinylidene derivatives of thiazolidin-4-ones: Methods of synthesis and biological activity (review). Chem. Heterocycl. Compd. 2015, 50, 1515-1527. [CrossRef]

15. Devprakash, D.; Bhoi, U.A. A complete review of thiazolidine-4-ones. J. Pharm. Rese. 2011, 4, 2436-2440.

16. Singh, S.P.; Parmar, S.S.; Raman, K.; Stenberg, V.I. Chemistry and biological activity of thiazolidinones. Chem. Rev. 1981, 81, 175-203. [CrossRef]

17. Cunico, W.; Gomes, C.R.B.; Vellasco, W.T., Jr. Chemistry and biological activities of 1,3-Thiazolidin-4-ones. Mini Rev. Org. Chem. 2008, 5, 336-344. [CrossRef]

18. Zapol'skii, V.A.; Namyslo, J.C.; Gjikaj, M.; Kaufmann, D.E. Chemistry of polyhalogenated nitrobutadienes, 14: Efficient synthesis of functionalized (Z)-2-allylidenethiazolidin-4-ones. Beilstein J. Org. Chem. 2014, 10, 1638-1644. [CrossRef]

19. Ibis, C.; Sayil, C.; Deniz, N.G. 1,3,4,4-Tetrachloro-4-(4-chlorophenylsulfanyl)-2-nitrobuta-1,3-diene. Acta Crystallogr. Sect. E Struct. Rep. Online 2006, 62, 800. [CrossRef]

20. Ishida, T.; In, Y.; Inoue, M.; Ueno, Y.; Tanaka, C.; Hamanaka, N. Structural elucidation of epalrestat(ONO-2235), a potent aldose reductase inhibitor, and isomerization of its double bonds. Tetrahedron Lett. 1989, 30, 959-962. [CrossRef]

21. Delgado, P.; Quiroga, J.; Cobo, J.; Low, J.N.; Glidewell, C. Supramolecular structures of four (Z)-5-arylmethylene-2-thioxothiazolidin-4-ones: Hydrogen-bonded dimers, chains of rings and sheets. Acta Crystallogr. Sect. C Cryst. Struct. Commun. 2005, 61, o477-o482. [CrossRef]

22. Opletalova, V.; Dolezel, J.; Králová, K.; Peško, M.; Kuneš, J.; Jampilek, J. Synthesis and characterization of (z)-5-Arylmethylidene-rhodanines with photosynthesis-inhibiting properties. Molecules 2011, 16, 5207-5227. [CrossRef]

23. Cutshall, N.S.; O'Day, C.; Prezhdo, M. Rhodanine derivatives as inhibitors of JSP-1. Bioorganic Med. Chem. Lett. 2005, 15, 3374-3379. [CrossRef]

24. Havrylyuk, D.; Zimenkovsky, B.; Vasylenko, O.; Zaprutko, L.; Gzella, A.; Lesyk, R. Synthesis of novel thiazolone-based compounds containing pyrazoline moiety and evaluation of their anticancer activity. Eur. J. Med. Chem. 2009, 44, 1396-1404. [CrossRef] [PubMed]

25. Zidar, N.; Tomašić, T.; Šink, R.; Rupnik, V.; Kovač, A.; Turk, S.; Patin, D.; Blanot, D.; Martel, C.C.; Dessen, A.; et al. Discovery of novel 5-Benzylidenerhodanine and 5-Benzylidenethiazolidine-2,4-dione inhibitors of MurD ligase. J. Med. Chem. 2010, 53, 6584-6594. [CrossRef] [PubMed]

26. Zapol'skii, V.A.; Namyslo, J.C.; Gjikaj, M.; Kaufmann, D.E. Chemistry of polyhalogenated nitrobutadienes, 15: Synthesis of novel 4-nitro-3-amino-1H-pyrazole-5-carbaldehydes and pyrazolo[3,4-f]indazole-4,8-diones. Heterocycles 2016, 93, 628-646. [CrossRef]

27. Potkin, V.I.; Zapol'Skii, V.A.; Knizhnikov, V.A.; Kaberdin, R.V.; Yanuchok, A.A.; Petkevich, S.K. Azolyl derivatives of nitrohalobutadienes: V. A new route to functionally substituted benzazetines. Russ. J. Org. Chem. 2001, 37, 689-694. [CrossRef]

28. Zapol'Skii, V.A.; Namyslo, J.C.; de Meijere, A.; Kaufmann, D.E. Chemistry of polyhalogenated nitrobutadienes, 10: Synthesis of highly functionalized heterocycles with a rigid 6-amino-3-azabicyclo[3.1.0]hexane moiety. Beilstein J. Org. Chem. 2012, 8, 621-628. [CrossRef]

29. Zapol'skii, V.A.; Potkin, V.I.; Nechai, N.I.; Kaberdin, R.V.; Pevzner, M.S. Azolyl derivatives of nitrohalobutadienes. I. Reaction of 1,1-bis(benzotriazol-1-yl)-2-nitrotrihalo-1,3-budadienes with $N_{-}, N_{,} N_{-}$, and N,O-Nucleophiles. Russ. J. Org. Chem. 1997, 33, 1461-1467.

30. Potkin, V.I.; Kaberdin, R.V. Benzazetines and their derivatives (review). Chem. Heterocycl. Compd. 2007, 43, 1493-1511. [CrossRef]

31. Ryder, S. Compounds for Modulating rna Binding Proteins and Uses Therefor. WO 2010151799 A2, 29 December 2010. 
32. Kagiyama, I.; Kato, H.; Nehira, T.; Frisvad, J.C.; Sherman, D.H.; Williams, R.M.; Tsukamoto, S. Taichunamides: Prenylated indole alkaloids from aspergillus taichungensis (IBT 19404). Angew. Chem. Int. Ed. 2015, 55, 1128-1132. [CrossRef]

33. Kaufmann, D.E.; Zapol'Skii, V.; Namyslo, J.; Altug, C.; Gjikaj, M. Chemistry of polyhalogenated nitrobutadienes, 6: A new ring-closure approach to perfunctionalized 5-nitropyrimidines. Synthesis 2008, 2008, 304-310. [CrossRef]

34. Riabova, O.B.; Makarov, V.A.; Granik, V.G.; Párkányi, C. Transformations of 5-nitropyrimidines. J. Heterocycl. Chem. 2008, 45, 621-643. [CrossRef]

35. Otmar, M.; Masojídková, M.; Votruba, I.; Holý, A. Synthesis and antiproliferative activity of 2,6-diamino-9-benzyl-9-deazapurine and related compounds. Bioorganic Med. Chem. 2004, 12, 3187-3195. [CrossRef] [PubMed]

36. Thompson, M.D.; Cupps, T.L.; Wise, D.S.; Wotring, L.L.; Townsend, L.B. Synthesis and evaluation of 6-(dibromomethyl)-5-nitropyrimidines as potential antitumor agents. J. Med. Chem. 1997, 40, 766-770. [CrossRef] [PubMed]

37. Chae, M.-Y.; Swenn, K.; Kanugula, S.; Dolan, M.E.; Pegg, A.E.; Moschel, R.C. 8-Substituted O6-benzylguanine, substituted 6(4)-(benzyloxy)pyrimidine, and related derivatives as inactivators of human O6-alkylguanine-DNA alkyltransferase. J. Med. Chem. 1995, 38, 359-365. [CrossRef] [PubMed]

38. Provins, L.; Christophe, B.; Danhaive, P.; Dulieu, J.; Durieu, V.; Gillard, M.; Lebon, F.; Lengele, S.; Quere, L.; van Keulen, B. First dual M3 antagonists-PDE4 inhibitors: Synthesis and SAR of 4,6-diaminopyrimidine derivatives. Bioorganic Med. Chem. Lett. 2006, 16, 1834-1839. [CrossRef] [PubMed]

39. Mikerova, N.I.; Panisheva, E.K.; Nikolaeva, I.S.; Pushkina, T.V.; Fomina, A.N.; Granik, V.G. Derivatives of 2-methyl-3-ethoxycarbonyl-5-(5-nitropyrimidyl-4)oxyindole: Synthesis and antiviral activity. Khimiko Farmatsevticheskii Zhurnal 1991, 25, 35-36.

40. Zhang, H.; Schinazi, R.F.; Chu, C.K. Synthesis of neplanocin F analogues as potential antiviral agents. Bioorganic Med. Chem. 2006, 14, 8314-8322. [CrossRef] [PubMed]

41. Cryan, J.F.; Kelly, P.H.; Chaperon, F.; Gentsch, C.; Mombereau, C.; Lingenhoehl, K.; Froestl, W.; Bettler, B.; Kaupmann, K.; Spooren, W.P.J.M. Behavioral characterization of the novel GABAB receptor-positive modulator GS39783 (N,N'-Dicyclopentyl-2-methylsulfanyl-5-nitro-pyrimidine-4,6-diamine): Anxiolytic-like activity without side effects associated with baclofen or benzodiazepines. J. Pharmacol. Exp. Ther. 2004, 310, 952-963. [CrossRef]

42. Urwyler, S.; Pozza, M.F.; Lingenhoehl, K.; Mosbacher, J.; Lampert, C.; Froestl, W.; Koller, M.; Kaupmann, K. $\mathrm{N}, \mathrm{N}^{\prime}$-Dicyclopentyl-2-methylsulfanyl-5-nitro-pyrimidine-4,6-diamine (GS39783) and structurally related compounds: Novel allosteric enhancers of $\gamma$-Aminobutyric AcidB receptor function. J. Pharmacol. Exp. Ther. 2003, 307, 322-330. [CrossRef]

43. Gray, N.S.; Janne, P.; Choi, H.G.; Jang, J. Novel Pyrimidines as egfr Inhibitors and Methods of Treating Disorders. WO 2016105525 A3, 29 September 2016.

44. Tsvelikhovsky, D.; Buchwald, S.L. Synthesis of heterocycles via Pd-Ligand controlled cyclization of 2-Chloro-N-(2-vinyl)aniline: Preparation of carbazoles, indoles, dibenzazepines, and acridines. J. Am. Chem. Soc. 2010, 132, 14048-14051. [CrossRef]

45. Low, J.N.; Trilleras, J.; Domingo, J.C.; Marchal, A.; Glidewell, C. A three-dimensional hydrogen-bonded framework in 2-amino-4,6-bis[N-methyl-N-(4-methylphenyl)amino]pyrimidine-5-carbaldehyde and hydrogen-bonded sheets in 2-amino-4-(indolin-1-yl)-6-methoxypyrimidine-5-carbaldehyde. Acta Crystallogr. Sect. C Cryst. Struct. Commun. 2007, 63, 681-684. [CrossRef]

46. Kaufmann, D.E.; Zapol'Skii, V.; Namyslo, J.; Gjikaj, M. Chemistry of polyhalogenated nitrobutadienes, part 5: Synthesis and reactions of dichloromethyl nitrovinylidene ketones of heterocycles. Synlett 2007, 2007, 1507-1512. [CrossRef]

47. Bürgi, M.; Zapol'Skii, V.A.; Hinkelmann, B.; Köster, M.; Kaufmann, D.E.; Sasse, F.; Hauser, H.; Etcheverrigaray, M.; Kratje, R.; Bollati-Fogolín, M.; et al. Screening and characterization of molecules that modulate the biological activity of IFNs-I. J. Biotechnology 2016, 233, 6-16. [CrossRef] [PubMed]

48. Singh, M.; Kaufmann, D.; Zapol'skii, V.; Oehlmann, W. 4-Nitro-5-dichloromethylpyrazol Derivatives for the Treatment of Infectious Diseases. EP 2829536 A1, 28 January 2015. 
49. Bolger, G.; Roy, S.; Zapol'Skii, V.A.; Kaufmann, D.E.; Schnürch, M.; Mihovilovic, M.D.; Nandy, R.K.; Tegge, W. Targeting aphA: A new high-throughput screening assay identifies compounds that reduce prime virulence factors of Vibrio cholerae. J. Med Microbiol. 2016, 65, 678-687. [CrossRef]

50. Sergeev, G.; Roy, S.; Jarek, M.; Zapolskii, V.; Kaufmann, D.E.; Nandy, R.K.; Tegge, W. High-throughput screening and whole genome sequencing identifies an antimicrobially active inhibitor of Vibrio cholerae. BMC Microbiol. 2014, 14, 49. [CrossRef] [PubMed]

51. Naylor, M.; Stephens, M.A.; Stratford, I.J.; Keohane, A.; O’Neill, P.; Threadgill, M.D.; Webb, P.; Fielden, E.M.; Adams, G.E. Aziridinyl nitropyrroles and nitropyrazoles as hypoxia-selective cytotoxins and radiosensitizers. Anti Cancer drug Des. 1991, 6, 151-167.

52. Gehring, R.; Jensen-Korte, U.; Schallner, O.; Stetter, J.; Santel, H.-J.; Schmidt, R.R.; Behrenz, W. Herbizide und insektizide Mittel auf Basis von Pyrazol-Derivaten. EP 0200872 A1, 16 March 1985.

53. Maue, M.; Harschneck, T.; Fischer, R.; Hahn, J.J.; Decor, A.; Hallenbach, W.; Schwarz, H.-G.; Koebberling, J.; Huebsch, W.; Bretschneider, T. novel halogen-substituted compounds. WO 2016020441 A1, 11 February 2016.

54. Bruderer, H.; Richle, R.; Ruegg, R. Verfahren Zur Herstellung Von Pyrazolderivaten. DE 2250316 A1, 26 April 1973.

55. Ukrainets, I.; Bereznyakova, N.L.; Turaibei, I.A. 4-Hydroxy-2-quinolones 139. Synthesis, structure, and antiviral activity of $\mathrm{N}-\mathrm{R}$-amides of 2-hydroxy-4-oxo-4H-pyrido[1,2-a]pyrimidine-3-carboxylic acids. Chem. Heterocycl. Compd. 2008, 44, 50-63. [CrossRef] [PubMed]

56. Sturgeon, S.A.; Jones, C.; Angus, P.W.; Wright, C.E. Advantages of a selective $\beta$-isoform phosphoinositide 3-kinase antagonist, an anti-thrombotic agent devoid of other cardiovascular actions in the rat. Eur. J. Pharmacol. 2008, 587, 209-215. [CrossRef] [PubMed]

57. Yoshida, K.-I.; Nakayama, K.; Ohtsuka, M.; Kuru, N.; Yokomizo, Y.; Sakamoto, A.; Takemura, M.; Hoshino, K.; Kanda, H.; Nitanai, H.; et al. MexAB-OprM specific efflux pump inhibitors in pseudomonas aeruginosa. Part 7: Highly soluble and in vivo active quaternary ammonium analogue D13-9001, a potential preclinical candidate. Bioorganic Med. Chem. 2007, 15, 7087-7097. [CrossRef] [PubMed]

58. La Motta, C.; Sartini, S.; Mugnaini, L.; Simorini, F.; Taliani, S.; Salerno, S.; Marini, A.M.; Da Settimo, F.; Lavecchia, A.; Novellino, E.; et al. Pyrido[1,2-a]pyrimidin-4-one derivatives as a novel class of selective aldose reductase inhibitors exhibiting antioxidant activity. J. Med. Chem. 2007, 50, 4917-4927. [CrossRef]

59. Barbeau, O.R.; Cano, C.; Griffin, R.J.; Hardcastle, I.R.; Smith, G.C.M.; Richardson, C.; Clegg, W.; Harrington, R.W.; Golding, B.T. Quinolinone and pyridopyrimidinone inhibitors of DNA-dependent protein kinase. Org. Biomol. Chem. 2007, 5, 2670-2677. [CrossRef]

60. Yoshida, K.-I.; Nakayama, K.; Kuru, N.; Kobayashi, S.; Ohtsuka, M.; Takemura, M.; Hoshino, K.; Kanda, H.; Zhang, J.Z.; Lee, V.J.; et al. MexAB-OprM specific efflux pump inhibitors in pseudomonas aeruginosa. Part 5: Carbon-substituted analogues at the C-2 position. Bioorganic Med. Chem. 2006, 14, 1993-2004. [CrossRef] [PubMed]

61. Kaul, S.; Zapol'skii, V.A.; Brönstrup, M.; Karge, B.; Gjikaj, M.; Kaufmann, D.E. Novel trisubstituted benzo[ $h]$ quinolines: Synthesis, subsequent reactions and evaluation of the antimicrobial and antifungal Activity. Eur. J. Org. Chem. 2020. under submission.

62. Ol'dekop, Y.A.; Kaberdin, R.V.; Potkin, V.I. Synthesis and some reactions of alkyl 2-nitro-1,3,4,4-tetrachloro-1,3-butadienyl sulfides. Chem. Informationsdienst 1980, 11, 469-472. [CrossRef]

63. Abe, H.; Harayama, T.; Kobayashi, N.; Takeuchi, Y. Synthesis of zanthoxyline and its related compounds: Revision of the reported structure. Heterocycles 2010, 80, 873-877. [CrossRef]

64. Cheng, M.-J.; Lee, K.-H.; Tsai, I.-L.; Chen, I.-S. Two new sesquiterpenoids and anti-HIV principles from the root bark of Zanthoxylum ailanthoides. Bioorganic Med. Chem. 2005, 13, 5915-5920. [CrossRef]

65. Kumar, S.; Sikka, H.C.; Dubey, S.K.; Czech, A.; Geddie, N.; Wang, C.X.; Lavoie, E.J. Mutagenicity and tumorigenicity of dihydrodiols, diol epoxides, and other derivatives of benzo(f)quinoline and benzo(h)quinoline. Cancer Res. 1989, 49, 20-24.

66. Cao, F.-J.; Yang, R.; Lv, C.; Ma, Q.; Lei, M.; Geng, H.-L.; Zhou, L. Pseudocyanides of sanguinarine and chelerythrine and their series of structurally simple analogues as new anticancer lead compounds: Cytotoxic activity, structure-activity relationship and apoptosis induction. Eur. J. Pharm. Sci. 2015, 67, 45-54. [CrossRef]

67. Kelley, C.; Lu, S.; Parhi, A.; Kaul, M.; Pilch, D.S.; Lavoie, E.J. Antimicrobial activity of various 4- and 5-substituted 1-phenylnaphthalenes. Eur. J. Med. Chem. 2012, 60, 395-409. [CrossRef] 
68. Cheng, X.-b.; Cheng, C.-m.; Wang, R.-j.; Hao, J. Single-crystal cultivation and structure analysis of unstable 1-azido-2-chloro-4-nitrobenzene. Chin. J. Structural Chem. 2014, 33, 1801-1806.

69. Chisari, A.; Parisi, G.; Maccarone, E.; Perrini, G. Sulphinyl, sulphonyl, and sulphonium groups as leaving groups in aromatic nucleophilic substitutions. J. Chem. Soc. Perkin Trans. 2 1982, 2, 957. [CrossRef]

70. Kice, J.L.; Kasperek, G.J. Quantitative comparison of nucleophilic substitution at sulfonyl vs. sulfinyl sulfur. Hydrolysis of aryl .alpha.-disulfones in aqueous dioxane. J. Am. Chem. Soc. 1969, 91, 5510-5516. [CrossRef]

71. Kaberdin, R.V.; Potkin, V.I.; Ol'dekop, Y.A. Synthesis of 3-trichloromethyl-4,5-dichloroisothiazole from 2-nitropentachloro-1,3-butadiene. Dokl. Chem. 1988, 300, 173-175.

72. Kaberdin, R.V.; Potkin, V.I.; Ol'dekop, Y.A. The synthesis and some reactions of 3-trichloromethyl-4,5-dichloroisothiazole. Russ. J. Org. Chem. 1990, 26, 1347-1351.

73. Li, J.; Wakefield, B.D.; Ruble, J.C.; Stiff, C.M.; Romero, D.L.; Marotti, K.R.; Sweeney, M.T.; Zurenko, G.E.; Rohrer, D.C.; Thorarensen, A. Preparation of novel antibacterial agents. Replacement of the central aromatic ring with heterocycles. Bioorganic Med. Chem. Lett. 2007, 17, 2347-2350. [CrossRef] [PubMed]

74. Kaizerman, J.A.; Gross, M.I.; Ge, Y.; White, S.; Hu, W.; Duan, J.-X.; Baird, E.E.; Johnson, K.W.; Tanaka, R.D.; Moser, H.E.; et al. DNA binding ligands targeting drug-resistant bacteria: Structure, activity, and pharmacology. J. Med. Chem. 2003, 46, 3914-3929. [CrossRef] [PubMed]

75. Bürli, R.W.; Ge, Y.; White, S.; Baird, E.E.; Touami, S.M.; Taylor, M.; Kaizerman, J.A.; Moser, H.E. DNA Binding Ligands with Excellent Antibiotic Potency Against Drug-Resistant Gram-Positive Bacteria. Bioorganic Med. Chem. Lett. 2002, 12, 2591-2594. [CrossRef]

76. Nutz, E.; Zapol'skii, V.A.; Kaufmann, D.E. Chemistry of polyhalogenated nitrobutadienes, 7: A novel synthetic access to chlorinated nitrile oxides. Synthesis 2009, 16, 2719-2725. [CrossRef]

77. Byrappa, S.; Raj, M.H.; Kungyal, T.; N, N.U.K.; Salimath, B.P.; Rai, K.M. Synthesis and biological evaluation of novel isoxazolines linked via piperazine to 2- benzoisothiazoles as potent apoptotic agents. Eur. J. Med. Chem. 2017, 126, 218-224. [CrossRef]

78. Kumar, K.S.V.; Lingaraju, G.S.; Bommegowda, Y.K.; Vinayaka, A.C.; Bhat, P.; Kumara, C.S.P.; Rangappa, K.S.; Gowda, D.C.; Sadashiva, M.P. Synthesis, antimalarial activity, and target binding of dibenzazepine-tethered isoxazolines. RSC Adv. 2015, 5, 90408-90421. [CrossRef]

79. Pulkkinen, J.; Poutiainen, P.; Oravilahti, T.; Palvimo, J.; Perakyla, M.; Laatikainen, R.; Ihalainen, T. Non-Steroidal Compounds As Androgen Receptor Modulators. US 9278942B2 3 August 2016.

80. Flores, A.F.C.; Piovesan, L.A.; Souto, A.A.; Pereira, M.A.; Martins, M.A.P.; Balliano, T.L.; Da Silva, G.S. Synthesis in water and antimicrobial activity of 5-Trichloromethyl-4,5-dihydroisoxazoles. Synth. Commun. 2013, 43, 2326-2336. [CrossRef]

81. Zapol'skii, V.A.; Namyslo, J.C.; Adam, A.E.W.; Kaufmann, D.E. Chemistry of polyhalogenated nitrobutadienes, Part 1: A new synthesis of perfunctionalized 3-amino-4-nitrothiophenes. Heterocycles 2004, 63, 1281. [CrossRef]

82. Vogt, E.-J.; Zapolskii, V.A.; Nutz, E.; Kaufmann, D.E. Chemistry of polyhalogenated nitrobutadienes, part 11: Ipso-formylation of 2-chlorothiophenes under vilsmeier-haack conditions. Zeitschrift für Naturforschung $B$ 2012, 67, 285-294. [CrossRef]

83. Kaufmann, D.E.; Zapol'Skii, V.A.; Vogt, E.-J.; Gjikaj, M. Chemistry of polyhalogenated nitrobutadienes, 12: Synthesis of novel, highly substituted Bi- and tricyclic 5,6,7,8-tetrahydro-4H-chromen-5-ones. Heterocycles 2012, 86, 1431. [CrossRef]

84. Peterson, S.; Guenther, R.H.; Mitchell, D. Compositions and Methods of Treating Drug-Resistant Retroviral Infections. WO 2012079032, 14 June 2012.

85. Guenther, R.H.; Szewczyk, J.R. Antiviral Compounds and Methods of Use Thereof. WO 2011113060, 15 September 2011.

86. Dolman, H.; Kuipers, J. Nitrothiophenes, Fungicidal and/or Bactericidal Compositions Containing Nitrothiophenes, and Use Thereof. U.S. Patent 4451660 A, 29 May 1984. 
87. Buechel, K.H.; Hammann, I. Insecticidal and Acaricidal 2-Anilino-3-Nitrothiophenes. DE 1949915 A1, 15 April 1971.

88. Nishigaki, J.; Deguchi, J. Oxonol compound and silver halide photographic material. EP 0819977 B1, 4 January 2006.

Sample Availability: Samples are available from the first author.

(C) 2020 by the authors. Licensee MDPI, Basel, Switzerland. This article is an open access article distributed under the terms and conditions of the Creative Commons Attribution (CC BY) license (http://creativecommons.org/licenses/by/4.0/). 